\title{
VLT spectroscopy of low-metallicity emission-line galaxies: abundance patterns and abundance discrepancies ${ }^{\star}, \star \star$
}

\author{
N. G. Guseva ${ }^{1,2}$, Y. I. Izotov ${ }^{1,2,3}$, G. Stasińska ${ }^{3}$, K. J. Fricke ${ }^{1,4}$, C. Henkel ${ }^{1}$, and P. Papaderos ${ }^{5,6}$ \\ 1 Max-Planck-Institute for Radioastronomy, Auf dem Hügel 69, 53121 Bonn, Germany \\ e-mail: guseva@mao.kiev.ua \\ 2 Main Astronomical Observatory, Ukrainian National Academy of Sciences, Zabolotnoho 27, Kyiv 03680, Ukraine \\ ${ }^{3}$ LUTH, Observatoire de Meudon, 92195 Meudon Cedex, France \\ 4 Institute for Astrophysics, University of Göttingen, Friedrich-Hund-Platz 1, 37077 Göttingen, Germany \\ 5 Centro de Astrofísica da Universidade do Porto, Rua das Esteral, 4150-762 porto, Portugal \\ ${ }^{6}$ Department of Astronomy, Oskar Klein Centre, Stockholm University, 10691 Stockholm, Sweden \\ Received 9 December 2010 / Accepted 7 March 2011
}

\section{ABSTRACT}

\begin{abstract}
Context. We present deep spectroscopy of a large sample of low-metallicity emission-line galaxies.
Aims. The main goal of this study is to derive element abundances in these low-metallicity galaxies.

Methods. We analyze 121 VLT spectra of $\mathrm{H}$ II regions in 46 low-metallicity emission-line galaxies. Of these spectra 83 are archival VLT/FORS1+UVES spectra of $\mathrm{H}$ II regions in 31 low-metallicity emission-line galaxies that are studied for the first time with standard direct methods to determine the electron temperatures, the electron number densities, and the chemical abundances.

Results. The oxygen abundance of the sample lies in the range $12+\log \mathrm{O} / \mathrm{H}=7.2-8.4$. We confirm previous findings that Ne/O increases with increasing oxygen abundance, likely because of a higher depletion of oxygen in higher-metallicity galaxies. The Fe/O ratio decreases from roughly solar at the lowest metallicities to about one tenth of solar, indicating that the degree of depletion of iron into dust grains depends on metallicity. The $\mathrm{N} / \mathrm{O}$ ratio in extremely low-metallicity galaxies with $12+\log \mathrm{O} / \mathrm{H}<7.5$ shows a slight increase with decreasing oxygen abundance, which could be the signature of enhanced production of primary nitrogen by rapidly rotating stars at low metallicity. We present the first empirical relation between the electron temperature derived from [S III] $\lambda 6312 / \lambda 9069$ or $\left[\mathrm{N}_{\text {II }}\right] \lambda 5755 / \lambda 6583$ and the one derived from $[\mathrm{O}$ III] $\lambda 4363 / \lambda(4959+5007)$ in low-metallicity galaxies. We also present an empirical relation between $t_{\mathrm{e}}$ derived from $[\mathrm{O}$ II] $\lambda 3727 /(\lambda 7320+\lambda 7330)$ or $[\mathrm{S} \mathrm{II}] \lambda 4068 /(\lambda 6717+\lambda 6730)$ and $[\mathrm{O}$ III] $\lambda 4363 / \lambda(4959+5007)$. The electron number densities $N_{\mathrm{e}}(\mathrm{Cl}$ III $)$ and $N_{\mathrm{e}}\left(\mathrm{Ar}\right.$ IV) were derived in a number of objects and are found to be higher than $N_{\mathrm{e}}(\mathrm{O}$ II $)$ and $N_{\mathrm{e}}(\mathrm{S}$ II). This has potential implications for the derivation of the pregalactic helium abundance. In a number of objects, the abundances of $\mathrm{C}^{++}$and $\mathrm{O}^{++}$could be derived from recombination lines. Our study confirms the discrepancy between abundances found from recombination lines (RLs) and collisionally excited lines (CELs) and that $\mathrm{C} / \mathrm{O}$ increases with $\mathrm{O} / \mathrm{H}$.
\end{abstract}

Key words. galaxies: starburst - methods: observational - galaxies: abundances - galaxies: dwarf - galaxies: ISM

\section{Introduction}

Comprehensive studies of low-metallicity emission-line galaxies in the nearby universe are essential to investigate star formation and galaxy evolution with chemical conditions close to those in the early universe. In particular, studies of abundance ratios and their dependencies on metallicity in low-metallicity galaxies are important for studying the early chemical evolution of galaxies and the nucleosynthesis of massive stars in an environment characterized by a nearly pristine chemical composition. Improved statistics of low-metallicity galaxies, observed with high accuracy, are also important to put observational constraints on the primordial helium abundance, which is a key parameter for testing cosmological models.

We here continue our study of nearby (redshift $z \lesssim$ 0.1 ) star-forming galaxies with measured intensities of the [O III] $\lambda 4363$ emission line (Guseva et al. 2006; Izotov et al. 2006; Papaderos et al. 2006; Guseva et al. 2007; Papaderos et al. 2008). This line is important to directly determine the physical

$\star$ Based on observations collected at the European Southern Observatory, Chile. List of programs is shown in Table 1.

$\star \star$ Tables 2-8 and 10 and Figs. 1-4 are available in electronic form at http://www. aanda.org conditions and chemical abundances of the ionized interstellar medium.

There were many spectroscopic surveys in the past aiming to study element abundances of nearby low-metallicity emissionline galaxies (e.g. Kunth \& Sargent 1983; Campbell et al. 1986; Terlevich et al. 1991; Masegosa et al. 1994; Melbourne \& Salzer 2002). However, most of these spectroscopic data are not suitable for our goals for various reasons, such as an insufficient quality of the data, the nonlinearity of the detectors in observations before the 1990ies, and the limited wavelength coverage.

In this paper, we consider archival data from the $8.2 \mathrm{~m}$ Very Large Telescope (VLT) obtained in the period 2000-2008 with the spectrographs FORS1 and UVES, which allow us to compile a sample of 83 high-quality spectra of metal-poor $\mathrm{H}$ II regions. We merge this sample with 15 archival VLT/FORS1+UVES spectra of two BCD galaxies, SBS 0335-052E and SBS 0335-052W (Izotov et al. 2009), and VLT/FORS2 spectra of $23 \mathrm{H}$ II regions in 12 low-metallicity emission-line galaxies from Guseva et al. (2009). We thus obtain a large VLT sample of $121 \mathrm{H}$ II regions, which includes to our knowledge all high-quality spectra from low-metallicity objects observed with the VLT/UVES+FORS instruments. They are used to study the abundance patterns of low-metallicity emission-line galaxies. 
Table 1. $\mathrm{H}$ in regions and galaxies observed with the VLT.

\begin{tabular}{|c|c|c|c|c|}
\hline Name & "RA (J2000.0) & 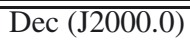 & Instrument & ESO Program \\
\hline UM 254 & $00: 31: 34.2$ & $-02: 09: 18.0$ & UVES & 71.B-0055(A) \\
\hline UM 283 & 00:51:49.6 & $+00: 33: 55.4$ & UVES & 71.B-0055(A),70.B-0717(A) \\
\hline NGC 346 & 00:59:06.0 & $-72: 10: 14.5$ & FORS low+medium resolution & 69.C-0203(A) \\
\hline NGC 456 & $01: 13: 53.3$ & $-73: 17: 49.8$ & FORS low+medium resolution & 69.C-0203(A) \\
\hline UM 133 & $01: 44: 41.3$ & $+04: 53: 24.3$ & UVES & 68.B-0310(A),70.B-0717(A) \\
\hline UM 382 & 01:58:09.5 & $-00: 06: 37.1$ & UVES & 70.B-0717(A) \\
\hline UM 408 & $02: 11: 23.5$ & $+02: 20: 32.0$ & UVES & 70.B-0717(A) \\
\hline UM 417 & 02:19:30.2 & $-00: 59: 16.2$ & UVES & 70.B-0717(A) \\
\hline UM 420 & $02: 20: 54.5$ & $+00: 33: 24.0$ & FORS low+medium resolution & 69.C-0203(A) \\
\hline MRK 600 & $02: 51: 04.5$ & $+04: 27: 15.0$ & UVES & 70.B-0717(A) \\
\hline CAM 0357-3915 & 03:59:08.9 & $-39: 06: 25.0$ & FORS low+medium resolution & 69.C-0203(A) \\
\hline TOL 0513-393 & $05: 15: 19.8$ & $-39: 17: 41.0$ & FORS low+medium resolution & 69.C-0203(A) \\
\hline TOL 0618-402 & $06: 20: 02.5$ & $-40: 18: 09.0$ & UVES & 70.B-0717(A) \\
\hline HE $2-10$ & $08: 36: 15.2$ & $-26: 24: 34.3$ & UVES & 073.B-0283(A), 081.C-0113(A) \\
\hline NGC 3125 & $10: 06: 33.3$ & $-29: 56: 07.5$ & UVES & 081.C-0113(A) \\
\hline MRK 1259 & $10: 38: 33.6$ & $-07: 10: 14.3$ & UVES & 073.B-0283(A) \\
\hline MRK 1271 & $10: 56: 09.1$ & $+06: 10: 22.0$ & UVES & 081.C-0113(A) \\
\hline POX 4 & $11: 51: 11.5$ & $-20: 35: 58.8$ & UVES & 081.C-0113(A) \\
\hline TOL 1214-277 & $12: 17: 18.7$ & $-28: 02: 21.3$ & UVES, FORS medium resolution & 69.D-0174(A), 65.N-0642(A) \\
\hline TOL 65 & $12: 25: 47.9$ & $-36: 13: 45.9$ & FORS medium resolution & 65.N-0642(A) \\
\hline J1253-0312 & $12: 53: 05.9$ & $-03: 12: 58.5$ & UVES & 081.C-0113(A) \\
\hline NGC 5253 & $13: 39: 55.9$ & $-31: 38: 24.7$ & UVES & 70.C-0008(A), 073.B-0283(A) \\
\hline TOL 89 & $14: 01: 20.0$ & $-33: 04: 11.2$ & UVES & 073.B-0283(A) \\
\hline NGC 5408 & $14: 03: 18.4$ & $-41: 22: 50.1$ & UVES & 081.C-0113(A) \\
\hline TOL $1457-262$ & $15: 00: 28.9$ & $-26: 26: 50.0$ & UVES & 081.C-0113(A) \\
\hline TOL 1924-416 & $19: 27: 58.1$ & $-41: 34: 31.2$ & UVES & 081.C-0113(A) \\
\hline NGC $6822 \mathrm{~V}$ & $19: 44: 52.7$ & $-14: 43: 09.8$ & UVES & 081.C-0113(A) \\
\hline NGC $6822 \mathrm{~V}+\mathrm{X}$ & $19: 44: 54.5$ & $-14: 43: 02.9$ & FORS low+medium resolution & 69.C-0203(A) \\
\hline TOL 2138-405 & $21: 41: 21.8$ & $-40: 19: 06.0$ & FORS low+medium resolution & 69.C-0203(A) \\
\hline TOL 2146-391 & 21:49:48.2 & $-38: 54: 09.0$ & FORS low+medium resolution & 69.C-0203(A) \\
\hline PHL 293B & $22: 30: 36.6$ & $-00: 06: 37.3$ & UVES & 70.B-0717(A) \\
\hline TOL 2240-384 & $22: 43: 33.0$ & $-38: 10: 55.2$ & FORS low+medium resolution & 69.C-0203(A) \\
\hline UM 160 & $23: 24: 22.0$ & $-00: 06: 21.2$ & FORS low+medium resolution & 69.C-0203(A) \\
\hline
\end{tabular}

For comparison reasons we supplemented this sample with 109 low-metallicity emission-line galaxies from the HeBCD sample that were observed with different telescopes and collected by Izotov \& Thuan (2004) and Izotov et al. (2004) for the study of the primordial He abundance. The total sample consists of objects with low extinction and in general with a high equivalent width $E W(\mathrm{H} \beta)$ of the $\mathrm{H} \beta$ emission line, which suggests a young age of the respective starburst. It also includes almost all most metal-deficient galaxies known, which allows for the deepest insights into the abundance pattern of the lowestmetallicity galaxies available in the local Universe. Additionally, we compare abundance patterns of this sample with those of the galaxies from the Data Release 3 (DR3) of the Sloan Digital Sky survey (SDSS) by Izotov et al. (2006) with lower quality spectra but higher metallicity, thus extending the range of oxygen abundances to higher values. $\mathrm{H}$ II regions in the above samples are selected mainly because of their high apparent brightness to obtain high signal-to-noise ratio spectra, and not considering their physical properties, such as the $\mathrm{H} \beta$ luminosity. However, these differing selection criteria do not introduce biases. In particular, Izotov et al. (2011) have shown that the abundances and abundance ratios of luminous compact galaxies selected from the SDSS by their high $\mathrm{H} \beta$ luminosity do not differ from the samples selected by their apparent brightness.

This paper is organized as follows: in Sect. 2 we describe observations and data reduction. In Sect. 3 we comment on the physical parameters of the $\mathrm{H}$ II regions. In Sect. 4 we discuss the abundance patterns derived from the analysis of collisionally excited lines and present carbon and oxygen abundances obtained from the analysis of recombination lines. We summarize our conclusions in Sect. 5.

\section{Observations and data reduction}

From the ESO archive we selected VLT spectra of $\mathrm{H}$ II regions that were obtained with the spectrographs UVES (high resolution) and FORS1 (low and medium resolution). The list of the 31 emission-line galaxies to which these $\mathrm{H}$ II regions pertain as well as the two H II regions NGC 346 and NGC 456 in the Small Magellanic Cloud is given in Table 1 together with the equatorial coordinates, the spectrographs used and the identification number of the ESO program. The total number of spectra from these galaxies available for abundance determinations (and listed in Table 2) is 83 - larger than the list in Table 1. This is because in some galaxies several one-dimensional spectra of $\mathrm{H}$ II regions were extracted from two-dimensional frames. One galaxy, Tol1214-277, was observed with two different spectrographs. Furthermore, the entire objects from the ESO program 69.C-0203(A) were observed with low and medium resolution. Four galaxies were observed twice with VLT/UVES within two different ESO programmes. The images of the observed galaxies overlayed by the slit positions are shown in Fig. 1.

The raw spectra of the objects from the ESO archive as well as the calibration spectra were reduced using IRAF ${ }^{1}$. The details

1 IRAF is distributed by the National Optical Astronomy Observatory,
which is operated by the Association of Universities for Research
in Astronomy, Inc., under cooperative agreement with the National Science Foundation. 
of data reduction are described in Thuan \& Izotov (2005). The two-dimensional spectra were bias subtracted and flat-field corrected. We then used the IRAF software routines IDENTIFY, REIDENTIFY, FITCOORD, and TRANSFORM to perform wavelength calibration and correction for distortion and tilt for each frame. Night sky subtraction was performed using the routine BACKGROUND. The level of night sky emission was determined from the regions closest to the $\mathrm{H}$ II region which are free of stellar and nebular line emission, as well as of emission from foreground and background sources. Exceptions are the extended Small Magellanic Cloud (SMC) H II regions NGC 346 and NGC 456, for which no night sky subtraction was done. One-dimensional spectra were then extracted from each twodimensional frame using the APALL routine. Before extraction, distinct two-dimensional spectra of the same $\mathrm{H}$ II region were carefully aligned using the spatial locations of the brightest part in each spectrum, so that spectra were extracted at the same positions in all subexposures. We have summed the individual spectra from each subexposure after removal of the cosmic ray hits.

The high-resolution spectra of $\mathrm{H}$ II regions observed with the UVES spectrograph are shown in Fig. 2, while low-resolution and medium-resolution FORS1 spectra are shown in Figs. 3 and 4 , respectively. All these figures are available in electronic form only. Emission-line fluxes were measured using the IRAF SPLOT routine. The line flux errors include statistical errors derived with SPLOT from non-flux-calibrated spectra, in addition to errors introduced by the absolute flux calibration, which we set to $1 \%$ of the line fluxes, according to the uncertainties of absolute fluxes of relatively bright standard stars (Oke 1990; Colina \& Bohlin 1994; Bohlin 1996; Izotov \& Thuan 2004). These errors will be later propagated into the calculation of the electron temperatures, the electron number densities, and ionic and the element abundances. Given a function $f(x, y, \ldots, z)$, the uncertainty $\sigma(f)$ is calculated as

$\sigma(f)=\sqrt{\left(\frac{\mathrm{d} f}{\mathrm{~d} x}\right)^{2} \sigma(x)^{2}+\left(\frac{\mathrm{d} f}{\mathrm{~d} y}\right)^{2} \sigma(y)^{2}+\ldots+\left(\frac{\mathrm{d} f}{\mathrm{~d} z}\right)^{2} \sigma(z)^{2}}$.

These errors do not include uncertainties introduced by the standard data reduction. In particular, one source of uncertainties comes from the division of object and standard star frames by the flat field frame. This is because the objects (especially those that are extended and have multiple knots) and the spectrophotometric standard star spectra are located in the frame at slightly different positions corresponding to slightly different intensities of the flat field. Additionally, atmospheric refraction may play a role resulting from the varying inclination of the spectra in the frame depending on airmass and position angle. Fringes in the red part of spectra introduce an additional source of uncertainties. The errors introduced by the standard data reduction and these fringes can be estimated by comparing the line intensities in different observations of the same $\mathrm{H}$ II region, although differences in slit positions and apertures may also contribute to differences in line intensities.

The corrections for underlying absorption in hydrogen lines and for extinction were performed following the procedure desribed in Guseva et al. (2009). We show in Tables 3-5 the extinction-corrected emission line fluxes relative to the $\mathrm{H} \beta$ fluxes along with the extinction coefficients $C(\mathrm{H} \beta)$, the observed fluxes $F(\mathrm{H} \beta)$ of the $\mathrm{H} \beta$ emission line, the equivalent widths $E W(\mathrm{H} \beta)$ of the $\mathrm{H} \beta$ emission line, and the equivalent widths $E W$ (abs) of the hydrogen absorption lines. All these tables are available in electronic form only. Table 3 contains the UVES observations, Table 4 the low-resolution, and Table 5 the medium-resolution FORS observations.

To constitute a VLT sample, that is as comprehensive as possible, we added to the data described above 15 archival VLT/FORS1+UVES spectra from the two BCD galaxies SBS 0335-052E and SBS 0335-052W studied before by Izotov et al. (2009) and 23 VLT/FORS2 spectra from 12 galaxies selected mainly from Data Release 6 (DR6) of the Sloan Digital Sky Survey (SDSS) and studied by Guseva et al. (2009). Our resulting VLT sample thus contains 121 spectra of $\mathrm{H}$ II regions from 46 low-metallicity emission-line galaxies. Eighty-three of these are archival VLT/FORS1+UVES spectra that are analyzed for the first time. All these spectra were observed with the same telescope and reduced in the same way.

\section{Physical conditions in the $\mathbf{H}$ II regions}

Physical conditions and element abundances in the selected $\mathrm{H}$ II regions were derived with the atomic data from the references listed in Stasińska (2005).

\subsection{Electron temperatures}

Nineteen out of the thirty one $\mathrm{H}$ II regions observed with VLT/UVES (Table 3) have detectable [S III] $\lambda 6312$ and [S III] $\lambda 9069$ emission lines in their spectra. This gives us the opportunity to determine the electron temperature $T_{\mathrm{e}}(\mathrm{S}$ III) directly from the spectra. In Fig. 5 we compare the electron temperatures $t_{\mathrm{e}}\left(\mathrm{O}\right.$ III) and $t_{\mathrm{e}}(\mathrm{S}$ III $)\left(t_{\mathrm{e}}=10^{-4} T_{\mathrm{e}}\right)$ obtained from

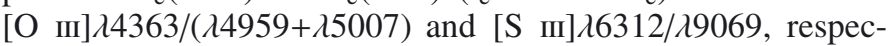
tively. The $\mathrm{H}$ in region data from VLT/UVES, including those for SBS 0335-052E from Izotov et al. (2009), are shown by stars. An exception are the three galaxies, which are represented by open circles. One of them (bottom left corner in both panels) belongs to the galaxy He 2-10E (UVES, ESO program 081.C-0113(A)). Its spectrum is noisy with a very weak and uncertain [O III] $\lambda 4363$ line intensity. The other two, in the upper right corner, belong to Tol 1214-277 (UVES, ESO program 69.D-0174(A)) and UM 133H (UVES, ESO program 68.B-0310(A)). At the redshift of Tol 1214-277 two absorption night-sky lines ( $\lambda 9300.6$ and 29303.9 ) coincide with the brightest part of the profile of the [S III] $\lambda 9069$ emission line, reducing its flux by $\sim 20 \%$ so that $t_{\mathrm{e}}(\mathrm{S}$ III) is overestimated (the point is actually outside the figure, which is indicated by an arrow). In UM133, the [S III] $\lambda 9069$ emission line is affected by the night sky line at 19118 . All our VLT/UVES and FORS data shown in Figs. 5-11 are presented in Table 10.

Additionally, we plot as red dots the data from Izotov et al. (2006) for the SDSS DR3. Out of more than 300 emission-line galaxies selected from the SDSS DR3 because they have a detectable $[\mathrm{O}$ III] $\lambda 4363$ line, we show here only the 27 galaxies for which $F(\mathrm{H} \beta)$ is higher than $2 \times 10^{-14} \mathrm{erg} \mathrm{s}^{-1} \mathrm{~cm}^{-2}$ and for which the errors in the [O III] $\lambda 4363$ fluxes are lower than $25 \%$. For comparison we also show data from Kehrig et al. (2006) (open blue rombs), Pérez-Montero \& Diaz (2003) (blue asterisks) and García-Rojas \& Esteban (2007) (purple triangles). The data by Pérez-Montero \& Diaz (2003) and Kehrig et al. (2006) were obtained from a spectroscopic analysis of $\mathrm{H}$ II regions in emission-line galaxies with ongoing star formation and the data by García-Rojas \& Esteban (2007) were derived for $\mathrm{H}$ II regions in our Galaxy. 

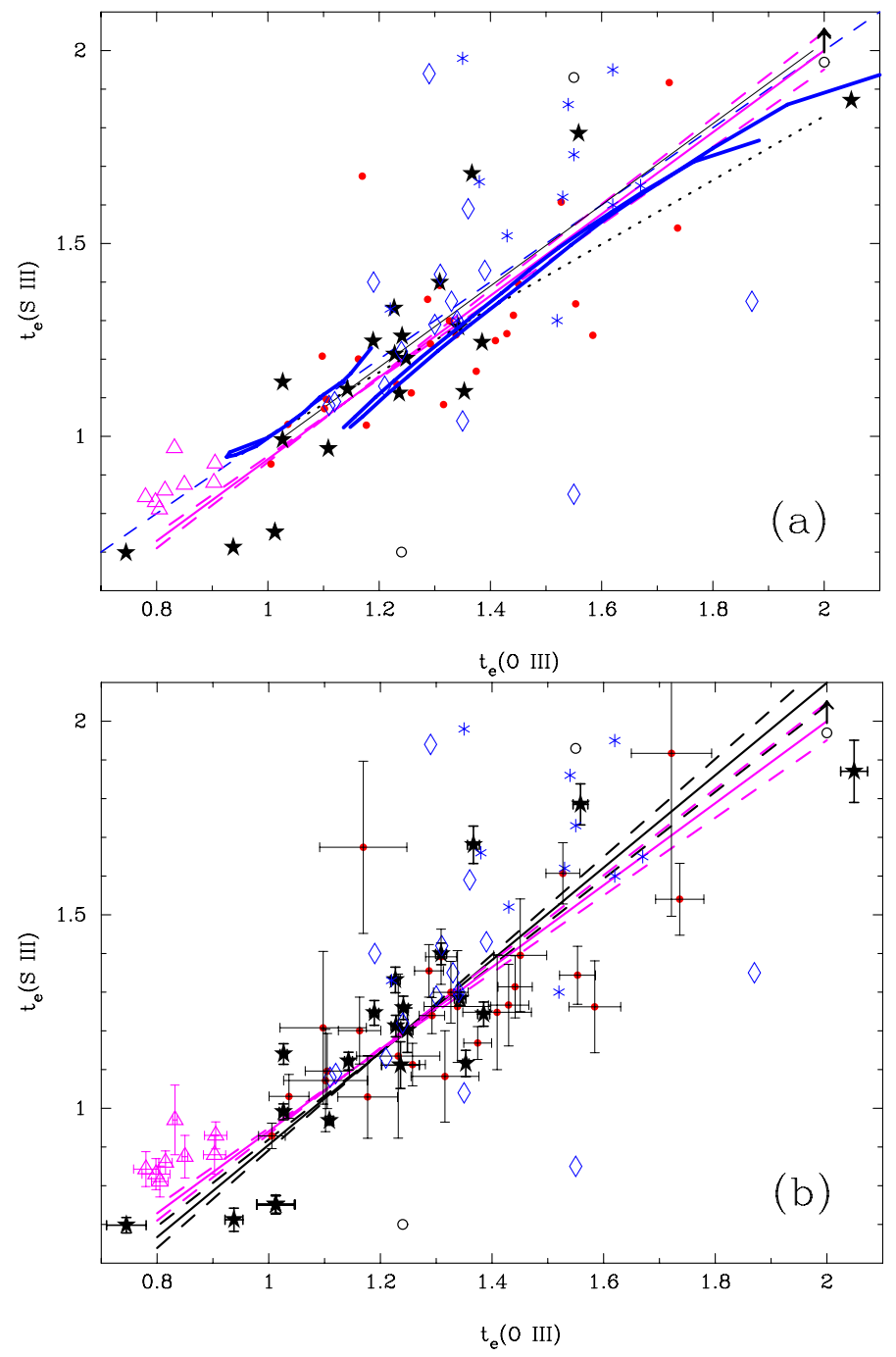

Fig. 5. Comparison of electron temperatures $t_{\mathrm{e}}\left(\mathrm{O}_{\text {III }}\right)\left(t_{\mathrm{e}}=10^{-4} T_{\mathrm{e}}\right)$ obtained from $[\mathrm{O}$ III] $] \lambda 4363 /(\lambda 4959+\lambda 5007)$ and $t_{\mathrm{e}}(\mathrm{S}$ III $)$ obtained from [S III] $\lambda 6312 / \lambda 9069$ emission-line ratios. VLT/UVES data are shown by stars, except for three objects with uncertain values, which are shown as open circles (see Sect. 3.1). Twenty-seven data points from SDSS DR3 (see text) are shown by red dots. Open blue diamonds, blue asterisks, and purple triangles correspond to data from Kehrig et al. (2006), PérezMontero \& Diaz (2003) and García-Rojas \& Esteban (2007), respectively. The dashed blue line in the upper panel connects locations of equal temperature. The thick blue lines (also in the upper panel) are the predicted $t_{\mathrm{e}}\left(\mathrm{S}\right.$ III) $-t_{\mathrm{e}}(\mathrm{O}$ III) relations for $\mathrm{H}$ II region models from Izotov et al. (2006). The dotted and thin solid black lines are the curves from Garnett (1992) and Pérez-Montero \& Diaz (2005), respectively. Regression lines (solid lines) and $1 \sigma$ alternatives (dashed lines) for all our data plus data from García-Rojas \& Esteban (2007) are shown by purple lines. In the lower panel the error bars are shown for the VLT, SDSS, and García-Rojas \& Esteban (2007) data. Additionally, regression lines for VLT+SDSS-only data are shown by black lines. (A color version of this figure is available in the online journal.)

The dashed blue line in the top panel connects points of equal temperatures. The thick lines show the predicted $t_{\mathrm{e}}(\mathrm{S}$ III) $t_{\mathrm{e}}(\mathrm{O}$ III) relation for $\mathrm{H}$ II region sequences of photoionization models with low, intermediate, and high metallicities $(12+$ $\log \mathrm{O} / \mathrm{H}=7.2,7.6$ and 8.2) from Izotov et al. (2006). The dotted and solid black lines display the model prediction from Garnett (1992) and Pérez-Montero \& Diaz (2005), respectively. The regression line including our data, the SDSS data, and the data
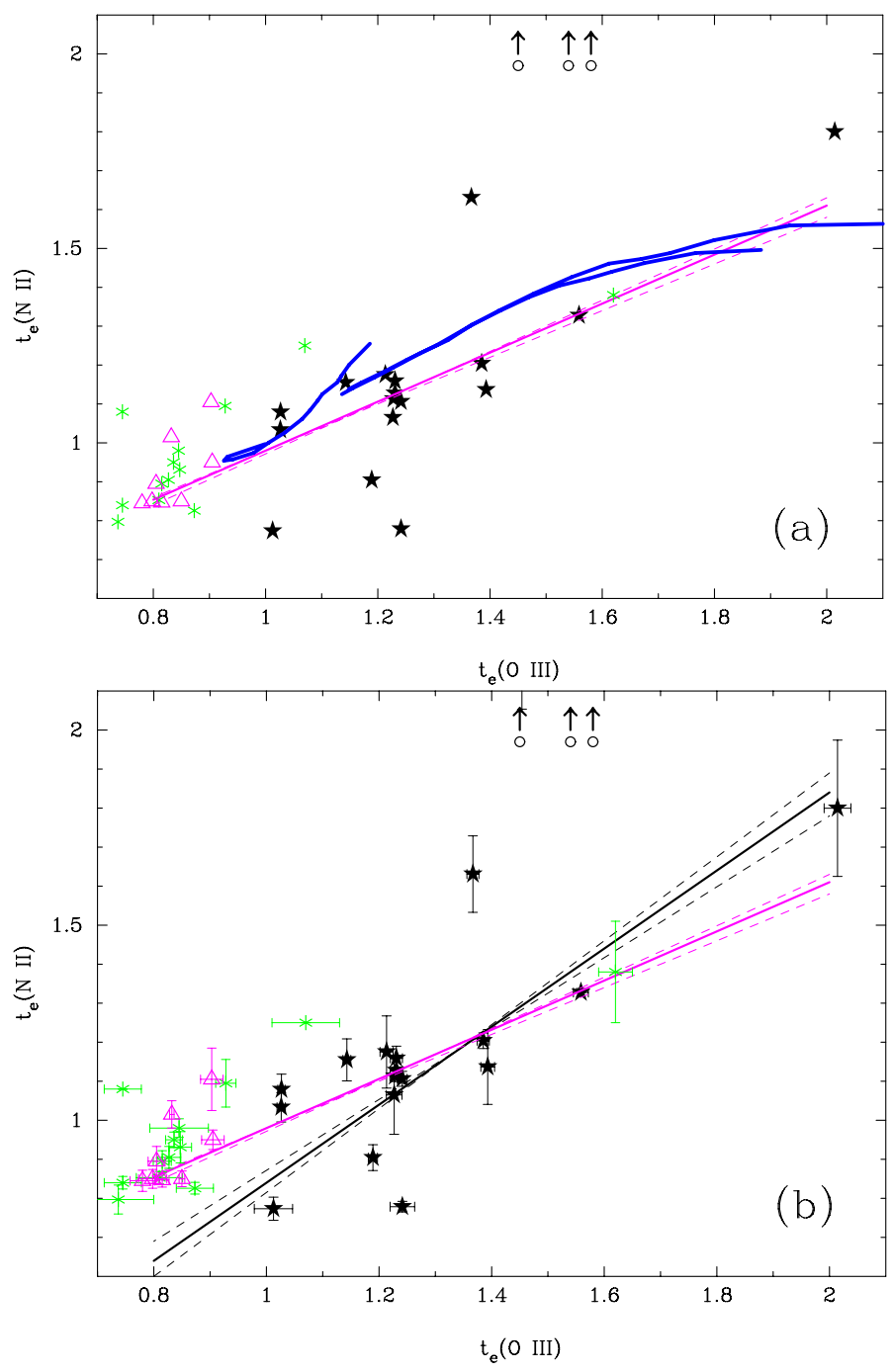

Fig. 6. Comparison of electron temperatures $t_{\mathrm{e}}(\mathrm{O}$ III $)\left(t_{\mathrm{e}}=10^{-4} T_{\mathrm{e}}\right)$ obtained from $[\mathrm{O}$ III] $] \lambda 4363 /(\lambda 4959+\lambda 5007)$ and $t_{\mathrm{e}}(\mathrm{N}$ II $)$ obtained from $[\mathrm{N}$ II] $] 5755 / \lambda 6583$ emission-line ratios. VLT/UVES and FORS data are shown by stars, except for two objects, which are shown as open circles (see text). Green asterisks and open purple triangles provide the data from Esteban et al. (2009) and García-Rojas \& Esteban (2007), respectively. The thick blue lines show the predicted $t_{\mathrm{e}}\left(\mathrm{O}_{\mathrm{II}}\right)-t_{\mathrm{e}}(\mathrm{O}$ III $)$ relation for $\mathrm{H}$ in region models from Izotov et al. (2006). Regression lines (solid lines) and $1 \sigma$ alternatives (dashed lines) for all data are shown by purple lines. The lower panel shows the error bars. Additionally, regression lines for our VLT-only data are shown by black lines. (A color version of this figure is available in the online journal.)

from García-Rojas \& Esteban (2007) are shown by purple lines. Its equation is

$t_{\mathrm{e}}(\mathrm{SIII})=(-0.1183 \pm 0.0321)+(1.0590 \pm 0.0281) \times t_{\mathrm{e}}(\mathrm{OIII})$.

The regression was computed using the maximum likelihood method by Press et al. (1992) that includes error bars on both axes. In the lower panel the error bars concerning the VLT, SDSS, García-Rojas \& Esteban (2007) data and additional regression lines for VLT and SDSS-only data are shown by black lines.

Clearly the data presented here define a much better empirical relation between $t_{\mathrm{e}}\left(\mathrm{S}\right.$ III) and $t_{\mathrm{e}}(\mathrm{O}$ III) than previous work on extragalactic $\mathrm{H}$ II regions. We note that the VLT/UVES data follow the same distribution as the best data from SDSS DR3. 


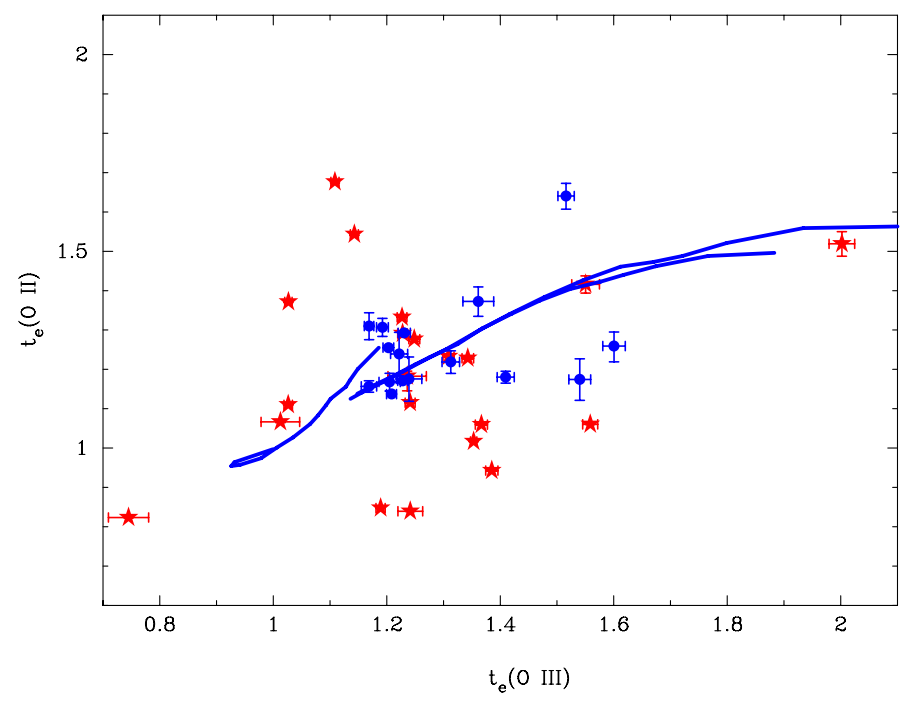

Fig. 7. Comparison of electron temperatures $t_{\mathrm{e}}\left(\mathrm{O}\right.$ III) $\left(t_{\mathrm{e}}=10^{-4} T_{\mathrm{e}}\right)$ derived from $[\mathrm{O}$ III] $] \lambda 4363 /(\lambda 4959+\lambda 5007)$ and $t_{\mathrm{e}}(\mathrm{O}$ II $)$ obtained from $[\mathrm{O}$ II $] \lambda 3727 /(\lambda 7320+\lambda 7330)$ emission-line ratios. VLT/UVES and FORS data are shown by red stars and blue filled circles, respectively. The thick blue lines represent the $t_{\mathrm{e}}\left(\mathrm{O}_{\text {II }}\right)-t_{\mathrm{e}}\left(\mathrm{O}_{\text {III }}\right)$ relations from Izotov et al. (2006). (A color version of this figure is available in the online journal.)

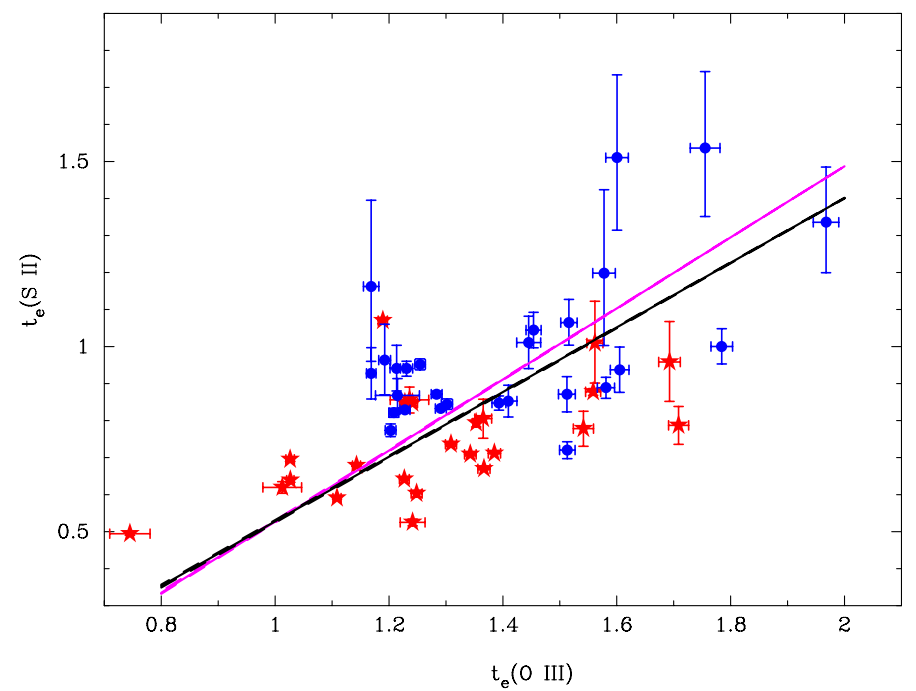

Fig. 8. Comparison of electron temperatures $t_{\mathrm{e}}\left(\mathrm{O}\right.$ III) $\left(t_{\mathrm{e}}=10^{-4} T_{\mathrm{e}}\right)$ obtained from $\left[\mathrm{O}\right.$ III] $\lambda 4363 /(\lambda 4959+\lambda 5007)$ and $t_{\mathrm{e}}\left(\mathrm{S}_{\mathrm{II}}\right)$ obtained from $[\mathrm{S}$ II] $] \lambda 4068 /(\lambda 6717+\lambda 6730)$ emission-line ratios. Symbols are the same as in Fig. 7. Regression lines for all data are represented by purple lines. Regression lines for the UVES-only data are shown by black lines. (A color version of this figure is available in the online journal.)

Unfortunately, for high electron temperatures there are only few data with reliable temperature determinations.

In metal-poor galaxies, [N II] $\lambda 5755$ is extremely weak. Only in 19 out of 121 VLT spectra could it be measured. To enlarge the range of temperatures we also added VLT/FORS data for the hot H II region in J0519+0007 from Guseva et al. (2009). In Fig. 6 we plot $t_{\mathrm{e}}(\mathrm{N}$ II) obtained from [N II] $] 25755 / \lambda 6583$ as a function of $t_{\mathrm{e}}(\mathrm{O}$ III). Data from our VLT sample are represented by stars. Tol 2240-384 (FORS medium and low resolution) and Tol 2138-405 No.3 (FORS medium resolution) are denoted by open circles with arrows, indicating that they are outside the plot owing to large $t_{\mathrm{e}}(\mathrm{N}$ II). Green asterisks display the data from
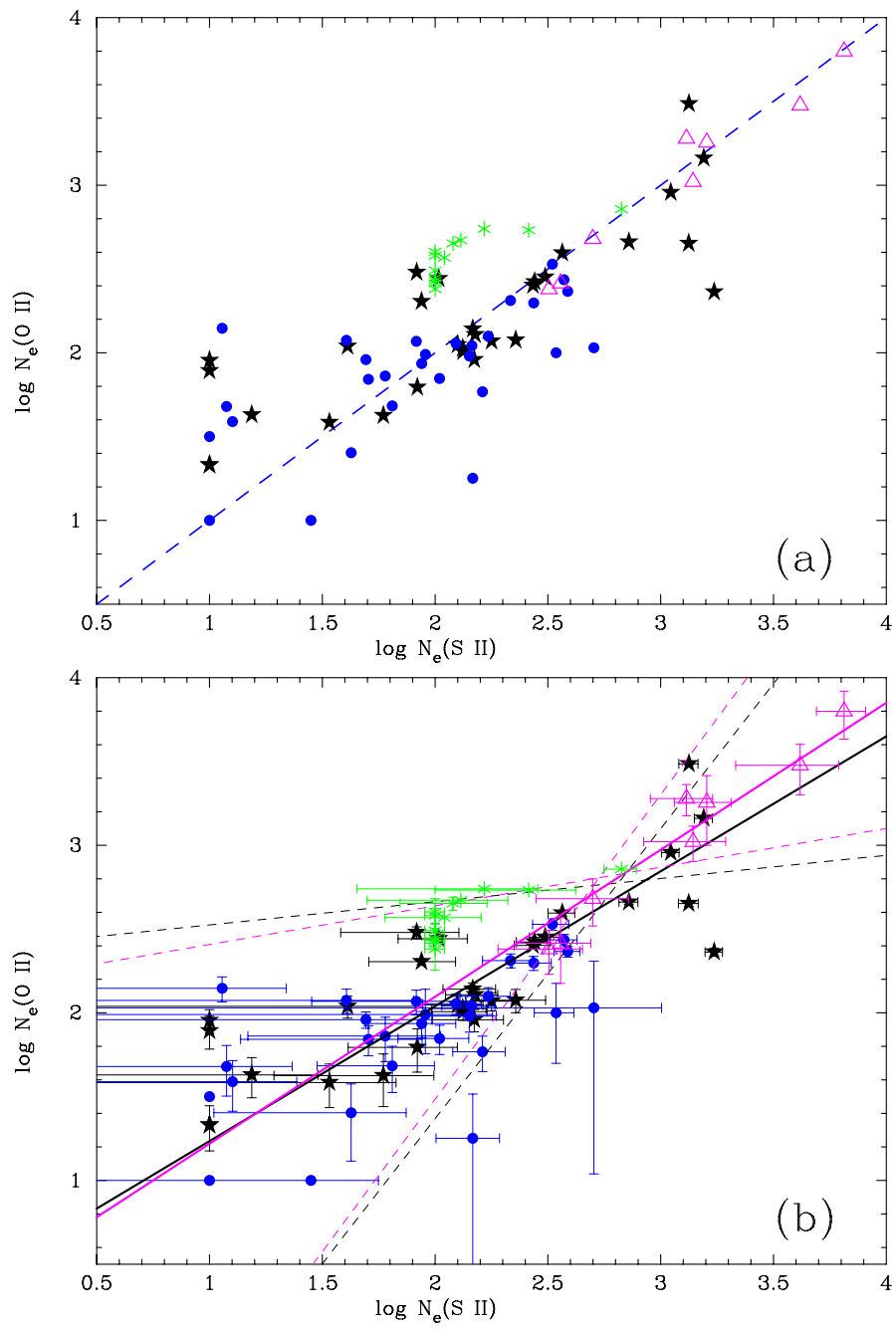

Fig. 9. $N_{\mathrm{e}}(\mathrm{O}$ II $)$ versus $N_{\mathrm{e}}(\mathrm{S}$ II $)$. Black stars: VLT-UVES; filled blue circles: FORS observations (medium resolution). Purple triangles: data from García-Rojas \& Esteban (2007), green asterisks: data from Esteban et al. (2009). The dashed blue line connects points of equal densities. The lower panel shows the error bars and regression lines and their $1 \sigma$ alternatives for all data (purple lines) and for the VLT-only data (black lines). (A color version of this figure is available in the online journal.)

Esteban et al. (2009). By open purple triangles we show the data from García-Rojas \& Esteban (2007).

The thick blue lines in the top panel of Fig. 6 represent the $t_{\mathrm{e}}(\mathrm{O}$ II $)-t_{\mathrm{e}}(\mathrm{O}$ III $)$ relation from the same Izotov et al. (2006) models as those considered in Fig. 5. It roughly follows the observational points, which show a substantial scatter. However, at the lowest temperatures considered, there seems to be an offset between the blue lines and our observational points.

The regression line for all data (upper panel) is given by the equation:

$t_{\mathrm{e}}(\mathrm{NII})=(0.3501 \pm 0.0158)+(0.6294 \pm 0.0138) \times t_{\mathrm{e}}(\mathrm{OIII})$.

The lower panel shows the error bars. Additionally, regression lines for our VLT-only data are shown by black lines. The

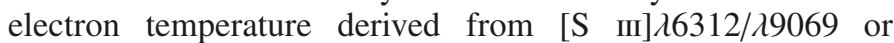
$\left[\begin{array}{ll}\mathrm{N} & \text { II }\end{array}\right] \lambda 5755 / \lambda 6583$ allowed us to obtain an empirical relation between those temperatures and the one derived from [O III $] \lambda 4363 / \lambda(4959+5007)$ for the first time for metal-poor galaxies. 

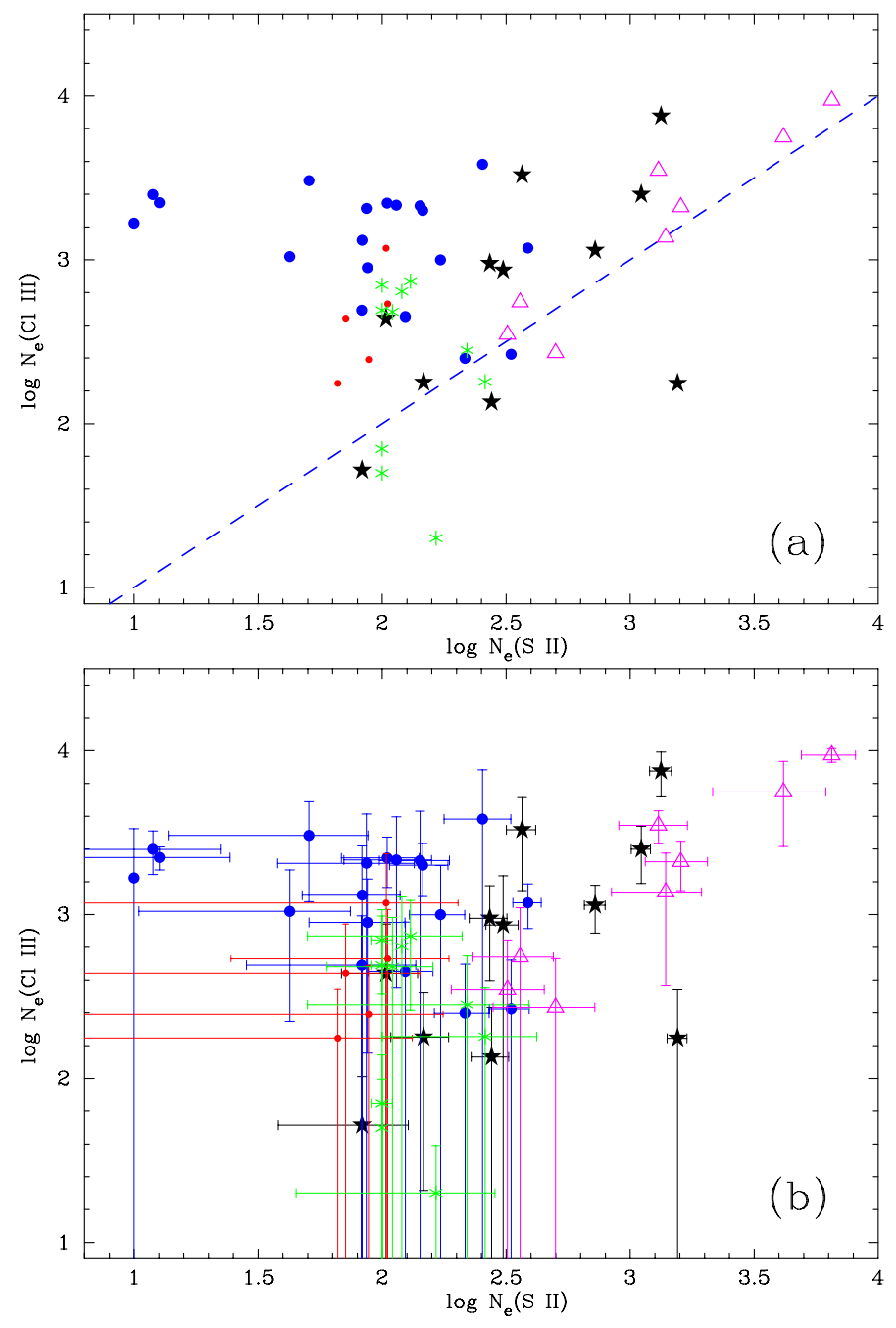

Fig. 10. $N_{\mathrm{e}}(\mathrm{Cl}$ III $)$ versus $N_{\mathrm{e}}(\mathrm{S}$ II). UVES, FORS and SDSS DR3 data are represented by black stars, filled blue circles, and red dots, respectively. Purple triangles: data from García-Rojas \& Esteban (2007), green asterisks: data from Esteban et al. (2009). The dashed blue line connects points of equal densities. The lower panel shows the error bars. (A color version of this figure is available in the online journal.)

In 21 UVES and 16 FORS spectra [O $\mathrm{II}] \lambda 3727$ and $\lambda \lambda 7320,7330$ lines have been measured. The [S II] $\lambda 4068$ and $\lambda \lambda 6717,6730$ lines are detected and measured in 24 UVES and 27 FORS spectra. These allow us to derive the electron temperatures $t_{\mathrm{e}}\left(\mathrm{O}_{\text {II }}\right)$ and $t_{\mathrm{e}}(\mathrm{S}$ II $)$ by direct methods. The results are shown in Figs. 7 and 8, where we compare those values with $t_{\mathrm{e}}(\mathrm{O}$ III). The UVES data are shown by red stars. Blue filled circles correspond to FORS data. The thick blue lines in Fig. 7 represent the $t_{\mathrm{e}}\left(\mathrm{O}_{\mathrm{II}}\right)-t_{\mathrm{e}}\left(\mathrm{O}_{\mathrm{III}}\right)$ relations from Izotov et al. (2006). Despite the large spread of points in Fig. 7 the VLT/UVES and FORS data follow the predicted $t_{\mathrm{e}}(\mathrm{O}$ II $)-t_{\mathrm{e}}(\mathrm{O}$ III $)$ relations (Izotov et al. 2006). The values of $t_{\mathrm{e}}(\mathrm{S}$ II) in Fig. 8 correlate well with $t_{\mathrm{e}}(\mathrm{O}$ III $)$, but are significantly below $t_{\mathrm{e}}\left(\mathrm{O}_{\text {II }}\right)$ in Fig. 7. Regression lines for all data in Fig. 8 are shown by purple lines and for the UVES-only data by black lines. The regression line for all the data displayed in Fig. 8 is given by the equation:

$$
t_{\mathrm{e}}(\mathrm{SII})=(-0.4323 \pm 0.0029)+(0.9596 \pm 0.0014) \times t_{\mathrm{e}}(\mathrm{OIII})
$$
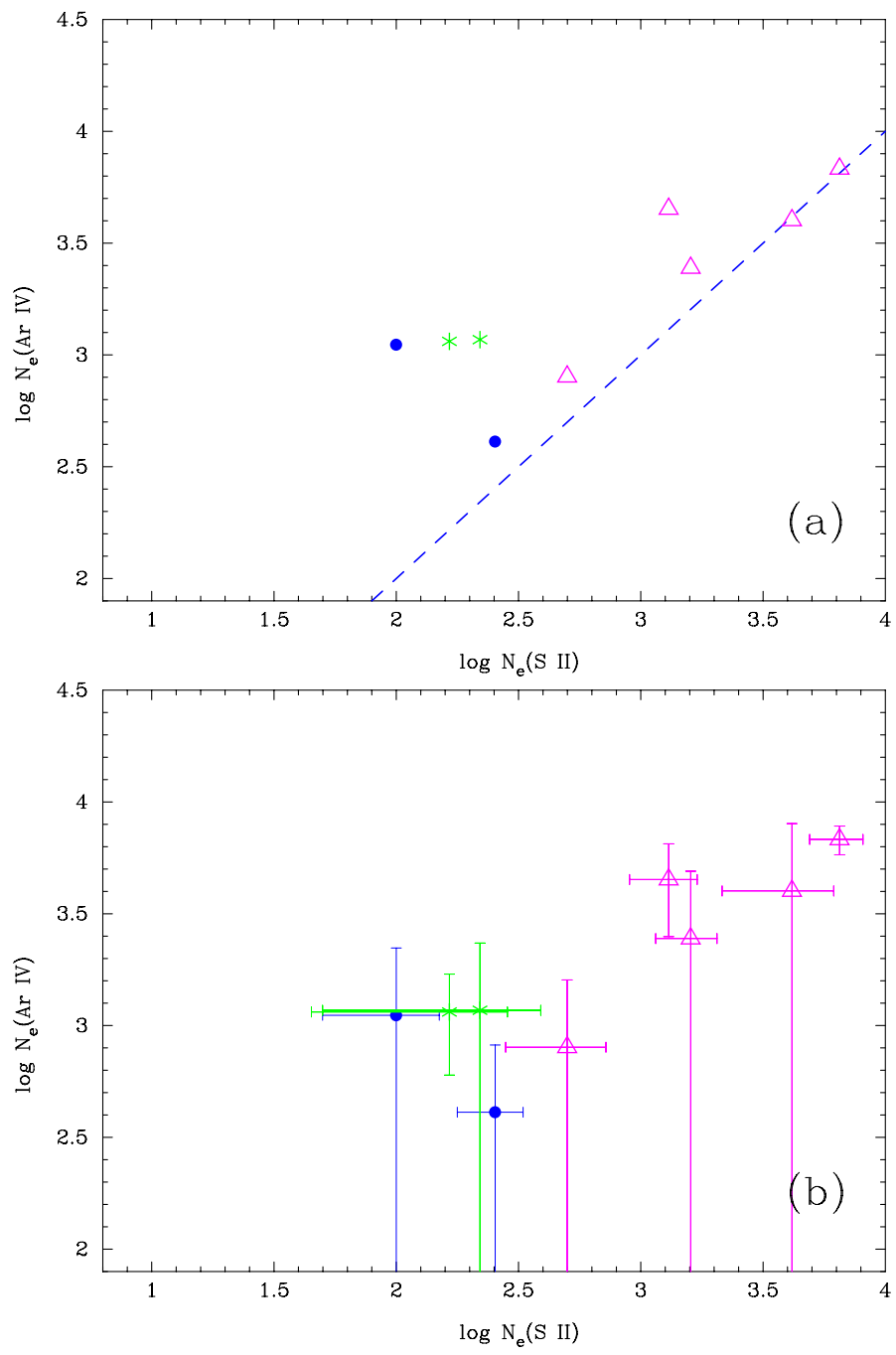

Fig. 11. $N_{\mathrm{e}}\left(\mathrm{Ar}\right.$ IV) versus $N_{\mathrm{e}}(\mathrm{S}$ II). FORS data are represented by filled blue circles. Purple triangles: data from García-Rojas \& Esteban (2007), green asterisks: data from Esteban et al. (2009). The dashed blue line connects points of equal densities. The lower panel shows the error bars. (A color version of this figure is available in the online journal.)

\subsection{The electron number densities}

For all galaxies the electron number densities $N_{\mathrm{e}}(\mathrm{S}$ II) were obtained from the [S II] $\lambda 6717 / \lambda 6731$ emission line ratio, except for regions where these emission lines are too weak to allow density determinations. For abundance determinations in those regions we adopt $N_{\mathrm{e}}=10 \mathrm{~cm}^{-3}$. The value of the electron number densities does not significantly affect the derived abundances in the low-density limit, which holds for the bulk of the $\mathrm{H}$ II regions considered here. The electron number densities $N_{\mathrm{e}}(\mathrm{S}$ II) derived from the $[\mathrm{S}$ II] $\lambda 6717 / \lambda 6731$ emission line ratio are given in Tables 6, 7 and 8.

The high signal-to-noise ratio and high spectral resolution of the spectra in the VLT sample allow us to measure the fluxes of the weak emission lines [Ar IV] $\lambda 4711,4740$ and $[\mathrm{Cl}$ III $] \lambda 5517,5537$ and to separate and measure the fluxes of the [O II] $\lambda 3726,3729$ lines to determine the electron number density. In many UVES spectra [O II] $\lambda 3726$ and [O II] $] 33729$ are seen as separate lines (for instance in UM 254, Fig. 2). However, for $\mathrm{H}$ II regions with broad components in their emission lines these 
two lines are poorly separated. In those cases the IRAF routine SPLOT was used for deblending. For all FORS medium resolution data the [O II] $\lambda 3726+3729$ lines were deblended by this IRAF software routine, adopting preliminary estimated widths of each of these emission lines.

The top panel of Fig. 9 shows $N_{\mathrm{e}}\left(\mathrm{O}\right.$ II) versus $N_{\mathrm{e}}(\mathrm{S}$ II). The data from UVES observations are shown by black stars, whereas medium-resolution FORS data are denoted by filled blue circles. We did not include SDSS data because of the very large errors in $N_{\mathrm{e}}(\mathrm{O}$ II $)$ determinations. Purple triangles represent data from García-Rojas \& Esteban (2007), green asterisks data from Esteban et al. (2009). The dashed blue line connects points of equal densities. The bottom panel of Fig. 9 shows the same data as the upper panel, but with error bars and regression lines.

The regression line for all data shown in Fig. 9 (lower panel, purple line) is presented by the equation

$$
\begin{aligned}
\log N_{\mathrm{e}}(\mathrm{OII})= & (0.3413 \pm 1.2484)+(0.8782 \pm 0.4798) \\
& \times \log N_{\mathrm{e}}(\mathrm{SII}) .
\end{aligned}
$$

The top panel of Fig. 10 shows $N_{\mathrm{e}}(\mathrm{Cl}$ III $)$ versus $N_{\mathrm{e}}(\mathrm{S}$ II $)$. UVES, FORS and SDSS DR3 data are represented by black stars, filled blue circles and red dots, respectively. Purple triangles are the data from García-Rojas \& Esteban (2007), green asterisks are the data from Esteban et al. (2009). The dashed blue line displays locations with equal densities. The error bars are shown in the lower panel. Only 14 SDSS galaxies could be used to derive $N_{\mathrm{e}}(\mathrm{Cl}$ III) due to our requirement that the error of the $[\mathrm{Cl}$ III $] \lambda 5517$ emission line flux is less than $25 \%$. Five of them are shown by red dots. For the remaining nine, the SDSS data allow only the derivation of a lower limit of $10 \mathrm{~cm}^{-3}$ (they are not shown in the figure).

The top panel of Fig. 11 shows $N_{\mathrm{e}}\left(\mathrm{Ar}\right.$ Iv) versus $N_{\mathrm{e}}(\mathrm{S}$ II). The FORS data are represented by filled blue circles. Purple triangles are the data from García-Rojas \& Esteban (2007), green asterisks are the data from Esteban et al. (2009). The dashed blue line is the place of equal densities. The lower panel shows the error bars and regression lines for all data. For $N_{\mathrm{e}}$ (Ar IV), we have a proper estimate for only two $\mathrm{H}$ II regions and a lower limit of $10 \mathrm{~cm}^{-3}$ for $50 \mathrm{H}$ II regions (not shown in the figure).

For all the objects $N_{\mathrm{e}}\left(\mathrm{O}\right.$ II) is very similar to $N_{\mathrm{e}}(\mathrm{S}$ II $)$ in the whole density range. Note that $\mathrm{H}$ II regions with $N_{\mathrm{e}}\left(\mathrm{S}_{\text {II }}\right)$ larger than $200 \mathrm{~cm}^{-3}$ represent only $\sim 10 \%$ of the entire VLT sample (Tables 6-8). On the other hand, $N_{\mathrm{e}}\left(\mathrm{Cl}\right.$ III) and $N_{\mathrm{e}}(\mathrm{Ar}$ IV) are systematically higher than $N_{\mathrm{e}}(\mathrm{S}$ II). This has no consequence on our determination of heavy element abundances below, but it will be important for considering the derivation of the pregalactic helium abundance.

\section{Element abundances}

\subsection{Derivation of the abundances}

The above analysis shows that the relations $t_{\mathrm{e}}(\mathrm{S}$ III $)$ vs. $t_{\mathrm{e}}(\mathrm{O}$ III), $t_{\mathrm{e}}\left(\mathrm{N}_{\text {II }}\right)$ and $t_{\mathrm{e}}\left(\mathrm{O}_{\text {III }}\right)$ vs. $t_{\mathrm{e}}\left(\mathrm{O}_{\text {III }}\right)$ derived from observations follow the same trends as the models by Izotov et al. (2006). Because the observed values are affected by large uncertainties in some cases and because many spectra lack the necessary data for the determination of $t_{\mathrm{e}}\left(\mathrm{S}\right.$ III) and $t_{\mathrm{e}}(\mathrm{N}$ II $)$, we prefer to use the same prescriptions as in Izotov et al. (2006).

To derive the abundances of $\mathrm{O}^{2+}, \mathrm{Ne}^{2+}$ and $\mathrm{Ar}^{3+}$, we adopted the temperature $T_{\mathrm{e}}(\mathrm{O}$ III) directly derived from the [O III] $\lambda 4363 /(\lambda 4959+\lambda 5007)$ emission-line ratio.
For $\mathrm{O}^{+}, \mathrm{N}^{+}, \mathrm{S}^{+}$, and $\mathrm{Fe}^{2+}$, we took the value of $t_{\mathrm{e}}(\mathrm{O}$ II $)$ obtained from $t_{\mathrm{e}}(\mathrm{O}$ III) using Eq. (14) in Izotov et al. (2006) that is shown below:

$$
\begin{aligned}
& t_{\mathrm{e}}(\mathrm{O} \text { II })=-0.577+t_{\mathrm{e}}(\mathrm{O} \text { III }) \times\left[2.065-0.498 t_{\mathrm{e}}(\mathrm{O} \text { III })\right], \\
& t_{\mathrm{e}}(\mathrm{O} \text { II })=-0.744+t_{\mathrm{e}}(\mathrm{O} \text { III }) \times\left[2.338-0.610 t_{\mathrm{e}}(\mathrm{O} \text { III })\right], \\
& t_{\mathrm{e}}(\mathrm{O} \text { II })=2.967+t_{\mathrm{e}}(\mathrm{O} \text { III }) \times\left[-4.797+2.827 t_{\mathrm{e}}(\mathrm{O} \text { III })\right],
\end{aligned}
$$

for $12+\log \mathrm{O} / \mathrm{H}=7.2$ and $t_{\mathrm{e}}(\mathrm{O}$ III $) \geq 1.14,12+\log \mathrm{O} / \mathrm{H}=7.6$ and $t_{\mathrm{e}}(\mathrm{O}$ III $) \geq 1.14$ and $12+\log \mathrm{O} / \mathrm{H}=8.2$ and $t_{\mathrm{e}}(\mathrm{O}$ III $) \leq 1.18$, respectively (see thick blue lines in Fig. 6a).

Similarly, to compute the $\mathrm{S}^{2+}, \mathrm{Cl}^{2+}$, and $\mathrm{Ar}^{2+}$ abundances we adopted the value of $t_{\mathrm{e}}(\mathrm{S}$ III $)$ obtained from $t_{\mathrm{e}}(\mathrm{O}$ III $)$ using Eq. (15) in Izotov et al. (2006):

$$
\begin{aligned}
& t_{\mathrm{e}}(\mathrm{S} \text { III })=-1.085+t_{\mathrm{e}}(\mathrm{O} \text { III }) \times\left[2.320-0.420 t_{\mathrm{e}}(\mathrm{O} \text { III })\right], \\
& t_{\mathrm{e}}(\mathrm{S} \text { III })=-1.276+t_{\mathrm{e}}(\mathrm{O} \text { III }) \times\left[2.645-0.546 t_{\mathrm{e}}(\mathrm{O} \text { III })\right], \\
& t_{\mathrm{e}}(\mathrm{S} \text { III })=1.653+t_{\mathrm{e}}(\mathrm{O} \text { III }) \times\left[-2.261+1.605 t_{\mathrm{e}}(\mathrm{O} \text { III })\right],
\end{aligned}
$$

for $12+\log \mathrm{O} / \mathrm{H}=7.2$ and $t_{\mathrm{e}}(\mathrm{O}$ III $) \geq 1.14,12+\log \mathrm{O} / \mathrm{H}=7.6$ and $t_{\mathrm{e}}(\mathrm{O}$ III $) \geq 1.14$ and $12+\log \mathrm{O} / \mathrm{H}=8.2$ and $t_{\mathrm{e}}(\mathrm{O}$ III $) \leq 1.18$, respectively (see thick blue lines in Fig. 5a).

Equations (6) and (7) (identical with Eqs. (14) and (15) in Izotov et al. 2006) are based on the $\mathrm{H}$ II region-photoionization models calculated for the given restricted (though large) ranges of input parameters and with the stellar atmosphere models by Smith et al. (2002) for the three values of the metallicity corresponding to $12+\log \mathrm{O} / \mathrm{H}=7.2,7.6$ and 8.2 (see thick blue lines in Figs. 6a and 5a). Therefore they hold for the restricted ranges of $12+\log \mathrm{O} / \mathrm{H}$ and $t_{\mathrm{e}}(\mathrm{O}$ III $)$ and we did not extrapolate them outside these ranges. The electron temperatures $t_{\mathrm{e}}\left(\mathrm{O}_{\mathrm{II}}\right)$ and $t_{\mathrm{e}}(\mathrm{S}$ III) depend on both the oxygen abundance $12+\log \mathrm{O} / \mathrm{H}$ and the electron temperature $t_{\mathrm{e}}(\mathrm{O}$ III). To derive these we fixed $t_{\mathrm{e}}(\mathrm{O}$ III $)$ and iteratively obtained $12+\log \mathrm{O} / \mathrm{H}$ and $t_{\mathrm{e}}(\mathrm{O}$ II $)$. First, the approximate value of $t_{\mathrm{e}}(\mathrm{O}$ II $)$ was derived from the first expression of Eq. (6) if $t_{\mathrm{e}}(\mathrm{O}$ III $) \geq 1.14$ or from the third expression of Eq. (6) if $t_{\mathrm{e}}(\mathrm{O}$ III $)<1.14$. Then the oxygen abundance was derived. Given the new iterative value of $12+\log \mathrm{O} / \mathrm{H}$, the new value of $t_{\mathrm{e}}(\mathrm{O}$ II $)$ was obtained. This process was continued until the convergence of both $12+\log \mathrm{O} / \mathrm{H}$ and $t_{\mathrm{e}}(\mathrm{O}$ II $)$ was achieved.

After that $t_{\mathrm{e}}(\mathrm{S}$ III) was derived. We used the linear interpolation of $t_{\mathrm{e}}(\mathrm{O}$ II $)$ and $t_{\mathrm{e}}(\mathrm{S}$ III $)$ between $12+\log \mathrm{O} / \mathrm{H}=7.2,7.6$ and 8.2 to the iterative value of $12+\log \mathrm{O} / \mathrm{H}$. The interpolation ranges for $12+\log \mathrm{O} / \mathrm{H}$ are between 7.2 and 8.2 for the $t_{\mathrm{e}}(\mathrm{O}$ III) range between 1.14 and 1.18 and are between 7.2 and 7.6 for $t_{\mathrm{e}}(\mathrm{O}$ III $)>1.18$. If the electron temperature $t_{\mathrm{e}}(\mathrm{O}$ III $)<1.14$, we adopted the equations for $12+\log \mathrm{O} / \mathrm{H}=8.2$ to derive $t_{\mathrm{e}}\left(\mathrm{O}_{\text {II }}\right)$ and $t_{\mathrm{e}}(\mathrm{S}$ III) (see Fig. 6a). If the iterated oxygen abundance was above the $12+\log \mathrm{O} / \mathrm{H}=8.2$, we used the third lines in Eqs. (6) and (7) for the determination of $t_{\mathrm{e}}(\mathrm{O}$ II $)$ and $t_{\mathrm{e}}(\mathrm{S}$ III). If the iterated $12+\log \mathrm{O} / \mathrm{H}<7.2$ we used the first lines in Eqs. (6) and (7) for the determination of $t_{\mathrm{e}}\left(\mathrm{O}_{\mathrm{II}}\right)$ and $t_{\mathrm{e}}(\mathrm{S}$ III $)$.

The electron temperatures $T_{\mathrm{e}}(\mathrm{O}$ III $), T_{\mathrm{e}}(\mathrm{O}$ II $), T_{\mathrm{e}}(\mathrm{S}$ III $)$ and electron number density $N_{\mathrm{e}}(\mathrm{S}$ II) are given in Table 6 (UVES observations) and Tables 7 and 8 (low- and medium-resolution FORS observations).

All the ionic abundances were computed using the lowdensity limit of the emissivities.

In all $\mathrm{H}$ II regions the abundances of $\mathrm{O}^{+}$and $\mathrm{O}^{2+}$ were obtained from the fluxes of the [O II] $\lambda 3727$ and [O III] $] 4959$, 5007 lines, respectively. We added the small fraction of the undetected $\mathrm{O}^{3+}$ ion to the oxygen abundance in high-excitation 


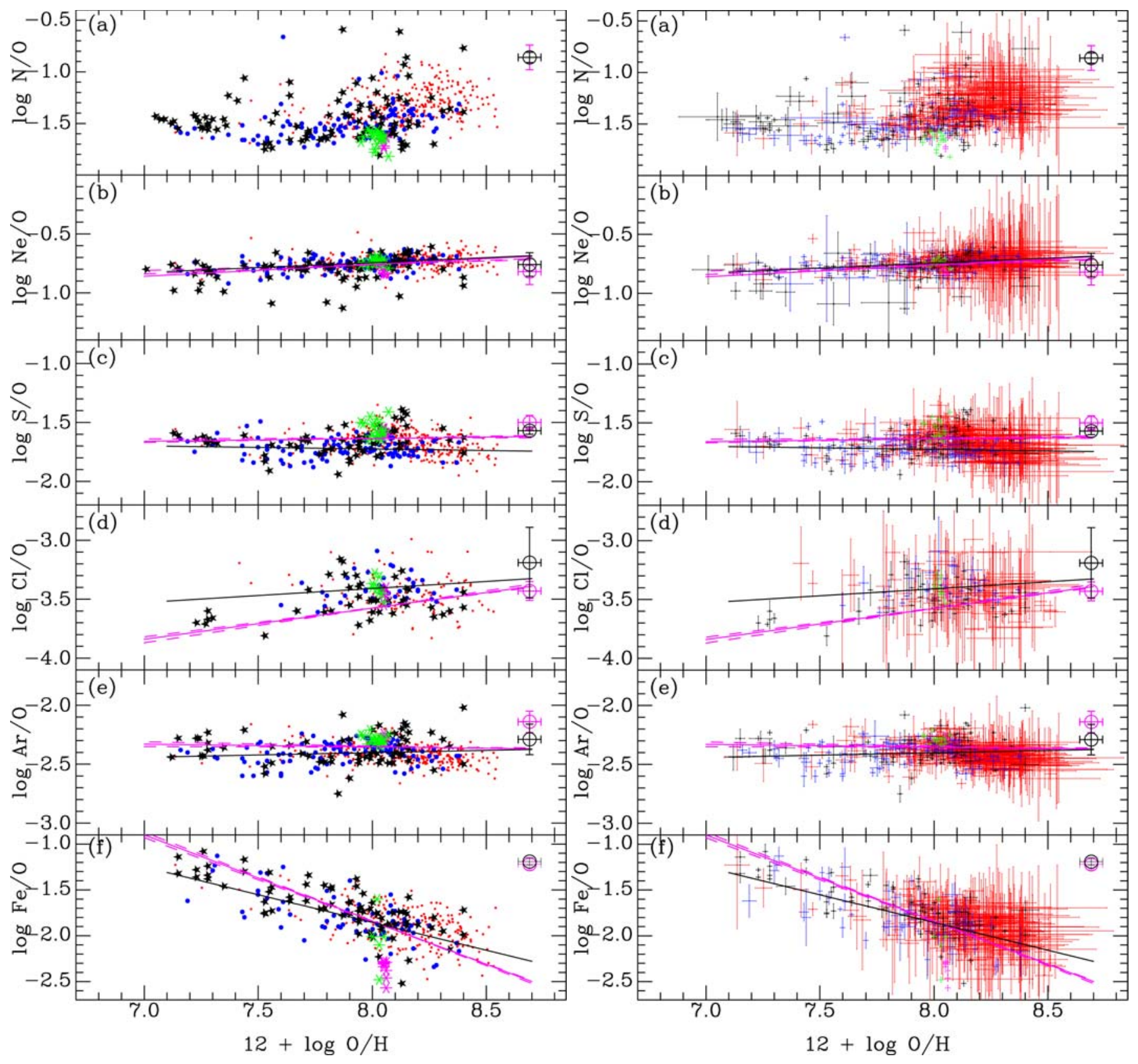

Fig. 12. $\log \mathrm{N} / \mathrm{O}$ a), $\log \mathrm{Ne} / \mathrm{O}$ b), $\log \mathrm{S} / \mathrm{O}$ c), $\log \mathrm{Cl} / \mathrm{O}$ d), $\log \mathrm{Ar} / \mathrm{O}$ e) and $\log \mathrm{Fe} / \mathrm{O}$ f) vs. oxygen abundance $12+\log \mathrm{O} / \mathrm{H}$ for our sample of 121 spectra (large stars). Several knots in H II regions NGC 346 and NGC 456 in the SMC are shown by green and purple large asterisks, respectively. Additionally we show the galaxies from the HeBCD sample of Izotov et al. (2004) and Izotov \& Thuan (2004) for the primordial He abundance determination (small filled blue circles) and the sample of $~ 300$ emission-line galaxies from the SDSS DR3 (Izotov et al. 2006) (red dots). The solar abundance ratios by Lodders (2003) and by Asplund et al. (2009) are indicated by the purple and black large open circles and the associated error bars. The straight black lines are linear regressions for the HeBCD sample by Izotov et al. (2006) and the purple ones are for all (except the SDSS data). The right panel shows the error bars. (A color version of this figure is available in the online journal.)

$\mathrm{H}$ II regions with $\mathrm{O}^{+} /\left(\mathrm{O}^{+}+\mathrm{O}^{2+}\right) \leq 0.1$ if the He II $\lambda 4686$ emission line was detected in their spectra. The total elemental abundances of the other elements were derived using the empirical ionization correction factors $(I C F$ s) of Izotov et al. (2006, their Eqs. (18)-(24)). Similar to the electron temperatures, we used the linear interpolation of $I C F \mathrm{~s}$ in the oxygen abundance range $12+\log \mathrm{O} / \mathrm{H}=7.2-8.2$ given the derived oxygen abundance for a particular $\mathrm{H}$ II region. If the oxygen abundance of the $\mathrm{H}$ II region was greater than 8.2 , we adopted $I C F$ s for $12+\log \mathrm{O} / \mathrm{H}=$ 8.2. Conversely, if the oxygen abundance of the $\mathrm{H}$ II region was less than 7.2 we adopted $I C F$ s for $12+\log \mathrm{O} / \mathrm{H}=7.2$. The ionic and total $\mathrm{O}, \mathrm{N}, \mathrm{Ne}, \mathrm{S}, \mathrm{Cl}, \mathrm{Ar}$, and $\mathrm{Fe}$ abundances derived from the forbidden emission lines are given in Tables 6-8 for the UVES and FORS observations. The quoted errors in the element abundances account for the uncertainties in the ionization correction factors.

\subsection{Abundance patterns from collisionally excited lines (CELs)}

The derived oxygen abundances for our VLT sample are found to cover the range of $12+\log \mathrm{O} / \mathrm{H} \sim 7.2-8.4$. In Fig. 12 left 
panel, we show the abundance ratios versus metallicities for the 121 spectra from our VLT sample (large stars). Several knots in H II regions NGC 346 and NGC 456 in the SMC are shown by green and purple large asterisks, respectively. We also plot the data from the HeBCD sample collected by Izotov et al. (2004) and Izotov \& Thuan (2004) (filled blue circles) and those from the DR3 of SDSS (Izotov et al. 2006) (red dots). The HeBCD sample possesses more than 100 high-quality spectra of low-metallicity galaxies for the primordial He abundance determination. The error bars are shown in the right panel of the figure.

In each panel, the solar abundance ratio by Lodders (2003) is indicated by the purple large open circle along with the associated error bar, whereas the solar abundance ratio by Asplund et al. (2009) is indicated by the black large open circle. The straight black and purple lines in Fig. 12 are the linear regressions obtained for the HeBCD sample taken from the paper by Izotov et al. (2006) and for all data (121 VLT + $109 \mathrm{HeBCD}$ spectra) respectively. The SDSS data have larger errors (Fig. 12, right panel) and therefore are not included in the regressions.

The regression lines for all data (excluding SDSS) are given by the equations

$$
\begin{array}{r}
\mathrm{Ne} / \mathrm{O}=[(-1.2850 \pm 0.0472)+(0.0656 \pm 0.0059)] \times X, \\
\left(n=249, \chi^{2}=1141\right), \\
\mathrm{S} / \mathrm{O}=[(-1.8310 \pm 0.0532)+(0.0244 \pm 0.0066)] \times X, \\
\left(n=203, \chi^{2}=6126\right), \\
\mathrm{Cl} / \mathrm{O}=[(-5.7188 \pm 0.0933)+(0.2680 \pm 0.0115)] \times X, \\
\left(n=98, \chi^{2}=1701\right), \\
\mathrm{Ar} / \mathrm{O}=[(-2.1739 \pm 0.0586)+(-0.0221 \pm 0.0071)] \times X, \\
\left(n=196, \chi^{2}=5146\right),
\end{array}
$$

$\mathrm{Fe} / \mathrm{O}=[(5.7180 \pm 0.0998)+(-0.9452 \pm 0.0121)] \times X$,

$$
\left(n=165, \chi^{2}=9032\right),
$$

where $X=12+\log \mathrm{O} / \mathrm{H}$.

Overall, we see in Fig. 12 a good overlap of our present VLT sample with the HeBCD and SDSS DR3 samples. The S/O, $\mathrm{Cl} / \mathrm{O}$ and $\mathrm{Ar} / \mathrm{O}$ abundance ratios are close to the distributions previously obtained by Izotov et al. (2006) and do not show any significant trend with oxygen abundance. Similar distributions for S/O and Ar/O have been obtained by Thuan et al. (1995), Izotov \& Thuan (1999) and van Zee \& Haynes (2006). We confirm the previous results using a significantly larger data base that encompasses 230 high-quality data. Some slight trends in $\mathrm{S} / \mathrm{O}, \mathrm{Cl} / \mathrm{O}$ and $\mathrm{Ar} / \mathrm{O}$ abundance ratios could imply a metallicity dependence of the $\alpha$-element production by massive stars. Variations of the explosion energy of Type II supernovae with low metallicity might also play a role (Kobayashi et al. 2006). However, Izotov et al. (2006) pointed out that abundance determinations for some elements such as $\mathrm{S}, \mathrm{Cl}$, and $\mathrm{Ar}$ could be uncertain because of the uncertainties in atomic data, e.g., in the rates of dielectronic recombination.

The ratio Ne/O (Fig. 12b) shows a slight increase with increasing $12+\log \mathrm{O} / \mathrm{H}$. This is likely explained by a stronger depletion of oxygen onto dust grains in higher-metallicity galaxies.

The most prominent trend was found by Izotov et al. (2006) for the $\mathrm{Fe} / \mathrm{O}$ abundance ratio, which decreases with increasing
$\mathrm{O} / \mathrm{H}$. While its value is nearly solar at the lowest metallicities, it drops by one order of magnitude at $12+\log \mathrm{O} / \mathrm{H}=8.5$. The interpretation of this trend put forward by Izotov et al. (2006) is that it is due to depletion of Fe onto dust grains, which becomes more important at higher metallicities.

The behavior of the N/O ratio is particularly interesting. Izotov \& Thuan (1999) and Izotov et al. (2006) have demonstrated that the dispersion in N/O in low-metallicity BCDs with $12+\log \mathrm{O} / \mathrm{H}<7.5-7.6$ is very low with a plateau value of $\log \mathrm{N} / \mathrm{O} \sim-1.6$. However, in a larger sample (Fig. 12a) some galaxies have a higher $\mathrm{N} / \mathrm{O}$ ratio at $12+\log \mathrm{O} / \mathrm{H}<7.6$. Several points with highest $\mathrm{N} / \mathrm{O}$ at $12+\log \mathrm{O} / \mathrm{H} \sim 7.5$ (Fig. 12a) belong to galaxies with low $E W(\mathrm{H} \beta)(10$ to $70 \AA)$. The notable exception is $\mathrm{J} 0519+0007$ with $E W(\mathrm{H} \beta)=240 \AA$. This is in line with the finding of Izotov et al. (2006) that the observed N/O increases as $E W(\mathrm{H} \beta)$ decreases. They showed that this enhancement could be due to high-density nitrogen-rich ejecta from massive stars during $\mathrm{W}-\mathrm{R}$ phases. The fact that no W-R features are detected in these HII regions is not a counterargument, because the W-R phase is expected to be short and the W-R features are weak in these low-metallicity systems.

We also find that the lower limit of $\mathrm{N} / \mathrm{O}$ in $\mathrm{H}$ II regions with lowest metallicity is higher than the above-mentioned plateau value of -1.6 , implying that there is some increase in $\mathrm{N} / \mathrm{O}$ with decreasing oxygen abundance at $12+\log \mathrm{O} / \mathrm{H}<7.5$ (Fig. 12a). If true, this tendency would agree with the prediction of primary nitrogen being produced by low-metallicity rotating stars, as found by Meynet \& Maeder (2002). Although these authors considered stellar models with a heavy element mass fraction $Z=10^{-5}$, which is significantly lower than the values of $Z \sim$ $0.0002-0.0005$ in the lowest-metallicity $\mathrm{H}$ II regions analyzed by us, it is possible that the interstellar medium in these galaxies enriched by the first metal-free stars memorizes this.

The two H II regions in the SMC considered by us, NGC 346 and NGC 456, have very low values of $\log \mathrm{Fe} / \mathrm{O}$, extending from -1.5 down to -2.6 . It would be interesting to investigate why these $\mathrm{H}$ II regions show such an extreme behavior. The remaining elements ( $\mathrm{Ne}, \mathrm{S}, \mathrm{Cl}, \mathrm{Ar})$ follow the same trends as in the other $\mathrm{H}$ II regions, with the exception of N/O. NGC 346 and NGC 456 have the lowest $\mathrm{N} / \mathrm{O}$ ratio among all the $\mathrm{H}$ II regions in our sample.

\subsection{Ionic abundances from recombination lines}

We detected and measured the fluxes of the recombination lines (RLs) $\mathrm{O}$ II $\lambda 4650$ and $\mathrm{C}_{\text {II }} \lambda 4267$ in eight medium-resolution FORS (out of 30) and 17 UVES (out of 31) H II region spectra. We detected only two out of eight $\mathrm{O}$ II recombination lines of multiplet 1. The multiplet consists of eight lines: $\lambda 4639$, $\lambda 4642, \lambda 4649, \lambda 4651, \lambda 4662, \lambda 4674, \lambda 4676$, and $\lambda 4696$. It is rarely possible to measure all the lines of this multiplet, and frequently it is necessary to estimate the intensities of the unobserved or blended lines. Owing to the insufficient resolution of the FORS medium resolution spectra, the O II $\lambda 4649$ and O II $\lambda 4651$ lines are blended into the pair $4649+4651$ named $\mathrm{O}$ II $\lambda 4650$. In the UVES spectra we measured the $\mathrm{O}$ II $\lambda 4649$ and $\lambda 4651$ lines separately, which were then co-added and named $\mathrm{O}$ II $\lambda$ 44650. The intensities of $\mathrm{O}_{\text {II }} \lambda 4650$ and $\mathrm{C}_{\text {II }} \lambda 4267$ lines were corrected for reddening using the extinction coefficient $\mathrm{C}(\mathrm{H} \beta)$ from Tables 3 and 5. Following Esteban et al. (2009) we calculated the abundance of $\mathrm{O}^{++} / \mathrm{H}^{+}$from O II $\lambda(4649+4651)$ and of $\mathrm{C}^{++} / \mathrm{H}^{+}$from $\mathrm{C}$ II $\lambda$ 4267. For the determination of the 
Table 9. Recombination lines: fluxes and ionic abundances.

\begin{tabular}{|c|c|c|c|c|c|c|}
\hline Name & $F(\mathrm{OII})^{a}$ & $F(\mathrm{CII})^{a}$ & $F(\mathrm{H} \beta)^{b}$ & $\begin{array}{c}\mathrm{O}^{++} / \mathrm{H}^{+c} \\
(\mathrm{RL}) \\
\end{array}$ & $\begin{array}{c}\mathrm{O}^{++} / \mathrm{H}^{+c} \\
(\mathrm{CEL})\end{array}$ & $\begin{array}{c}\mathrm{C}^{++} / \mathrm{H}^{+c} \\
(\mathrm{RL}) \\
\end{array}$ \\
\hline NGC 346a No. $1^{d}$ & $7.77 \pm 7.23$ & $<0.82$ & $517.00 \pm 0.40$ & $3.895 \pm 0.759$ & $0.888 \pm 0.021$ & $<0.165$ \\
\hline NGC 346a No. $2^{d}$ & $4.66 \pm 0.67$ & $<0.58$ & $381.30 \pm 0.34$ & $3.124 \pm 0.120$ & $0.916 \pm 0.022$ & $<0.160$ \\
\hline NGC $346 \mathrm{~b}$ No. $1^{d}$ & $5.49 \pm 1.28$ & $<0.75$ & $456.30 \pm 0.40$ & $3.083 \pm 0.153$ & $0.859 \pm 0.021$ & $<0.172$ \\
\hline NGC 456a No. $1^{d}$ & $2.69 \pm 0.65$ & $1.43 \pm 0.55$ & $377.60 \pm 0.33$ & $1.698 \pm 0.089$ & $0.703 \pm 0.017$ & $0.392 \pm 0.151$ \\
\hline NGC 456a No. $2^{d}$ & $2.31 \pm 0.77$ & $2.22 \pm 1.73$ & $231.70 \pm 0.26$ & $2.461 \pm 0.263$ & $0.857 \pm 0.022$ & $0.981 \pm 0.762$ \\
\hline SBS $0335-052 \mathrm{E}^{e}$ & & $3.20 \pm 2.04$ & $499.60 \pm 0.26$ & & $0.183 \pm 0.005$ & $0.245 \pm 0.156$ \\
\hline He $2-10 \mathrm{E}^{e}$ & $22.07 \pm 3.38$ & $<15.46$ & $3627.00 \pm 3.03$ & $1.538 \pm 0.017$ & $1.471 \pm 0.297$ & $<0.171$ \\
\hline NGC $3125^{e}$ & $10.54 \pm 2.18$ & $9.07 \pm 1.03$ & $863.30 \pm 0.49$ & $3.072 \pm 0.083$ & $1.507 \pm 0.034$ & $0.426 \pm 0.049$ \\
\hline Mrk $1259^{e}$ & $41.30 \pm 12.23$ & $<34.75$ & $1025.00 \pm 1.17$ & $8.917 \pm 0.202$ & $0.568 \pm 0.064$ & $<1.336$ \\
\hline Mrk $1271^{e}$ & $10.78 \pm 1.31$ & $8.24 \pm 2.82$ & $1368.00 \pm 0.89$ & $1.957 \pm 0.048$ & $0.820 \pm 0.019$ & $0.255 \pm 0.087$ \\
\hline $\operatorname{Pox} 4^{e}$ & $5.49 \pm 1.90$ & $3.30 \pm 0.99$ & $1457.00 \pm 0.77$ & $0.920 \pm 0.069$ & $1.031 \pm 0.024$ & $0.096 \pm 0.029$ \\
\hline Tol $1214-277^{d}$ & $1.76 \pm 0.49$ & $<0.22$ & $700.80 \pm 0.51$ & $0.521 \pm 0.027$ & $0.311 \pm 0.009$ & $<0.029$ \\
\hline Tol $1214-277^{e}$ & $<3.41$ & $<0.75$ & $700.80 \pm 0.52$ & $<1.096$ & $0.292 \pm 0.008$ & $<0.045$ \\
\hline $\mathrm{J} 1253-0312^{e}$ & $<3.29$ & $2.34 \pm 1.06$ & $1834.00 \pm 0.77$ & $<0.359$ & $0.869 \pm 0.020$ & $0.054 \pm 0.024$ \\
\hline NGC5253 No.C1 ${ }^{e}$ & $14.39 \pm 2.35$ & $11.95 \pm 1.84$ & $2559.00 \pm 1.61$ & $1.301 \pm 0.031$ & $1.195 \pm 0.027$ & $0.196 \pm 0.030$ \\
\hline NGC5253 No.C2 ${ }^{e}$ & $9.30 \pm 1.92$ & $9.88 \pm 1.97$ & $1019.00 \pm 0.98$ & $2.212 \pm 0.113$ & $1.487 \pm 0.035$ & $0.384 \pm 0.077$ \\
\hline NGC5253 No.P1 ${ }^{e}$ & $38.86 \pm 12.83$ & $28.99 \pm 9.42$ & $1954.00 \pm 2.03$ & $4.625 \pm 0.340$ & $0.729 \pm 0.011$ & $0.626 \pm 0.203$ \\
\hline NGC5253 No.P2 ${ }^{e}$ & $31.92 \pm 7.78$ & $23.38 \pm 4.84$ & $3265.00 \pm 2.63$ & $2.160 \pm 0.089$ & $1.194 \pm 0.027$ & $0.303 \pm 0.063$ \\
\hline NGC 5408 No. $1^{e}$ & $12.68 \pm 1.72$ & $7.20 \pm 1.01$ & $1194.00 \pm 0.78$ & $2.474 \pm 0.032$ & $0.939 \pm 0.022$ & $0.255 \pm 0.036$ \\
\hline NGC 5408 No. $2^{e}$ & $7.61 \pm 0.99$ & & $404.40 \pm 0.35$ & $4.729 \pm 0.084$ & $1.073 \pm 0.026$ & \\
\hline Tol $1457-262^{e}$ & $<3.25$ & $3.75 \pm 1.32$ & $733.00 \pm 0.71$ & $<1.218$ & $1.312 \pm 0.030$ & $0.213 \pm 0.075$ \\
\hline Tol1924-416 No. $1^{e}$ & $<6.77$ & $3.26 \pm 1.21$ & $988.55 \pm 1.06$ & $<1.568$ & $0.784 \pm 0.019$ & $0.140 \pm 0.052$ \\
\hline NGC $6822 \mathrm{~V}^{e}$ & $14.66 \pm 1.76$ & $6.94 \pm 1.05$ & $1643.00 \pm 0.73$ & $2.323 \pm 0.029$ & $1.265 \pm 0.027$ & $0.173 \pm 0.026$ \\
\hline NGC $6822 \mathrm{~V}$ No. $1^{d}$ & $1.36 \pm 0.57$ & $<0.13$ & $66.21 \pm 0.13$ & $5.539 \pm 0.197$ & $1.192 \pm 0.033$ & $<0.212$ \\
\hline Tol 2138-405 No. $1^{d}$ & $0.53 \pm 0.33$ & $<0.21$ & $58.06 \pm 0.12$ & $2.122 \pm 0.226$ & $0.855 \pm 0.022$ & $<0.382$ \\
\hline
\end{tabular}

Notes. ${ }^{(a)}$ In units $10^{-17} \mathrm{erg} \mathrm{s}^{-1} \mathrm{~cm}^{-2} .{ }^{(b)}$ In units $10^{-16} \mathrm{erg} \mathrm{s}^{-1} \mathrm{~cm}^{-2} .{ }^{(c)}$ In units $10^{-4}$. ${ }^{(d)}$ Medium resolution FORS data. ${ }^{(e)}$ UVES data.

$\mathrm{O}^{++}$abundances we used the prescriptions by Peimbert et al. (2005) to calculate the correction factor for the contribution of unmeasured lines of multiplet 1 for the non-LTE conditions. The intensities of the lines of multiplet 1 weakly depend on the electron density. We used the values of $N_{\mathrm{e}}(\mathrm{S}$ II $)$ to derive the correction factors. If taking a higher value of the density, as indicated by $N_{\mathrm{e}}\left(\mathrm{Cl}\right.$ III) (Fig. 10) and $N_{\mathrm{e}}$ (Ar IV) (Fig. 11), the RL abundances decrease by $\sim 5-15 \%$. The effective recombination coefficients were taken from Storey (1994) for O II RLs and from Davey et al. (2000) for C II RLs.

In Table 9 we present the observed fluxes of O II $\lambda 4650$, C II $\lambda 4267$ and $\mathrm{H} \beta$ emission lines, the ionic abundances of $\mathrm{O}^{++} / \mathrm{H}^{+}$obtained from RLs and CELs, and $\mathrm{C}^{++} / \mathrm{H}^{+}$obtained from RLs using the electron temperature derived from [O III] $\lambda 4363 /(\lambda 4959+\lambda 5007)$.

$\mathrm{O}^{++}$is the only ion for which both strong CELs and measured RLs are present in the optical range. In Fig. 13 we show the dependence of the abundance discrepancy factor ADF = $\log \mathrm{O}^{++}(\mathrm{RL}) / \mathrm{O}^{++}(\mathrm{CEL})$ on the observed $F(\mathrm{H} \beta)$. UVES data are shown by red stars, FORS medium-resolution data by filled blue circles. We determined, where possible, $\mathrm{ADF}$ and $\mathrm{C}^{++}(\mathrm{RL})$ and compared our results with those of other studies. Green asterisks represent the data from Esteban et al. (2009). There is no clear trend with $F(\mathrm{H} \beta)$ for $F(\mathrm{H} \beta)>10^{-13} \mathrm{erg} \mathrm{s}^{-1} \mathrm{~cm}^{-2}$ and the error bars are large. Many UVES cases are compatible with no discrepancy at all. However, half of the cases, especially the FORS and UVES data with $F(\mathrm{H} \beta)<10^{-13} \mathrm{erg} \mathrm{s}^{-1} \mathrm{~cm}^{-2}$, indicate an ADF clearly larger than zero. This is in line with what was found by Esteban et al. (2009) for a set of extragalactic $\mathrm{H}$ II regions as well with what is summarized by García-Rojas \& Esteban (2007) for galactic $\mathrm{H}$ in regions. We investigated the possible existence of a correlation between the ADF and any of the

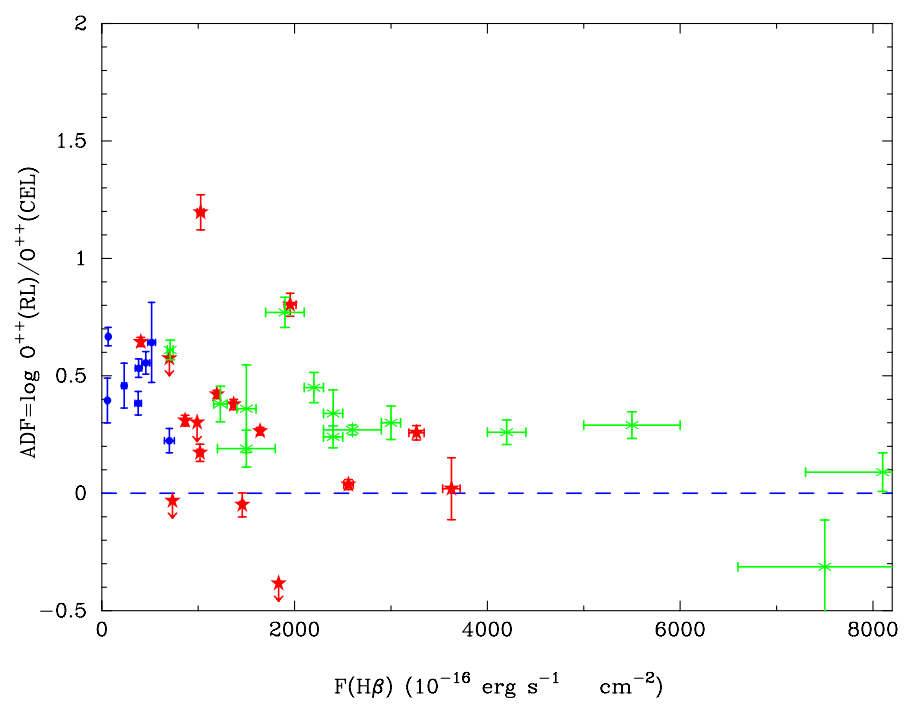

Fig. 13. Comparison of the abundance discrepancy factor ADF $=$ $\log \mathrm{O}^{++}(\mathrm{RL}) / \mathrm{O}^{++}(\mathrm{CEL})$ with observed $F(\mathrm{H} \beta)$. Data from UVES and FORS medium resolution observations are shown by red stars and filled blue circles. Green asterisks are the data from Esteban et al. (2009). (A color version of this figure is available in the online journal.)

following parameters: $C(\mathrm{H} \beta), \mathrm{O}^{+} /\left(\mathrm{O}^{+}+\mathrm{O}^{+}\right), \mathrm{O} / \mathrm{H} \quad(\mathrm{CEL})$, $\operatorname{FWHM}(\mathrm{H} \beta)$ and electron number density, hoping to find a clue for the origin of those ADFs. Like García-Rojas \& Esteban (2007), we found no evidence for any correlation whatsoever.

Whatever the reason for the abundance discrepancy may be, we can use the $\mathrm{C}^{++}(\mathrm{RL}) / \mathrm{O}^{++}(\mathrm{RL})$ ratio to achieve some insight into the behavior of the $\mathrm{C} / \mathrm{O}$ ratio. Figure 14 shows the values of $\log \mathrm{C}^{++}(\mathrm{RL}) / \mathrm{O}^{++}(\mathrm{RL})$ as a function of $12+\log \mathrm{O} / \mathrm{H}(\mathrm{CEL})$. 


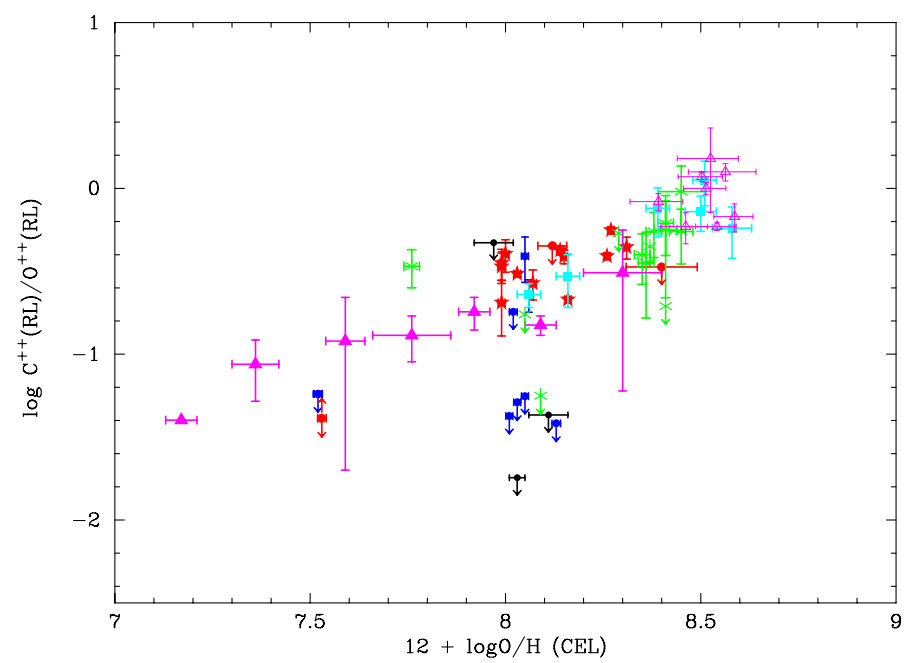

Fig. 14. Dependence of the ion abundance ratio $\log \mathrm{C}^{++}(\mathrm{RL}) / \mathrm{O}^{++}(\mathrm{RL})$ on $12+\log \mathrm{O} / \mathrm{H}(\mathrm{CEL})$. Our data are marked by the same symbols as in Fig. 13. Data from Garnett et al. (1995) and García-Rojas \& Esteban (2007) are shown by filled and open purple triangles, respectively. Green asterisks are the data from Esteban et al. (2009). Data from Garnett et al. (1999) and Kobulnicky \& Skillman (1998) are shown by light blue squares and black circles, respectively. (A color version of this figure is available in the online journal.)

The symbols are the same as in Fig. 13. We supplement our data with those from Garnett et al. (1995), where $\mathrm{C}^{++} / \mathrm{O}^{++}$are derived from the UV CELs and García-Rojas \& Esteban (2007), which are shown by filled and open purple triangles, respectively. Green asterisks are the data from Esteban et al. (2009). The data from Garnett et al. (1999) and Kobulnicky \& Skillman (1998) for the C III]1909 line are shown by light blue squares and black circles, respectively. We find that if $\mathrm{C}^{++}(\mathrm{RL}) / \mathrm{O}^{++}(\mathrm{RL})$ can be taken as a measure of $\mathrm{C} / \mathrm{O}$, the $\mathrm{C} / \mathrm{O}$ ratio increases with $\mathrm{O} / \mathrm{H}$. The only objects that do not follow this trend are the ones from our sample and from Esteban et al. (2009) and Kobulnicky \& Skillman (1998), for which only $1 \sigma$ upper limits to the $C_{\text {II }} \lambda 4267$ line flux are estimated. The two leftmost points are for Tol 1214-277. One of these points is from the UVES spectrum, where only upper limits to the $F\left(\mathrm{O}_{\text {III }}\right)$ and $F\left(\mathrm{C}_{\text {II }}\right)$ are obtained, and the second point is from the FORS medium-resolution spectrum. Four points at $12+\log \mathrm{O} / \mathrm{H} \sim 8.05$ belong to NGC $6822 \mathrm{~V}$ No. 1 and to three knots in the SMC H II region NGC 346. Note, that for NGC 346 no night sky subtraction was made.

\section{Summary}

We have presented an analysis of archival VLT/FORS1+UVES spectroscopic observations of a large sample of low-metallicity emission-line galaxies. The whole sample, which contains some data from our previous papers (Izotov et al. 2009; and Guseva et al. 2009), consists of 121 spectra, out of which 83 are analyzed for the first time. For comparison, we also used data from SDSS DR3 studied by Izotov et al. (2006) and 109 spectra from the HeBCD sample observed with different telescopes and collected by Izotov \& Thuan (2004) and Izotov et al. (2004) for the study of the primordial $\mathrm{He}$ abundance.

Our main results are as follows:

1. The oxygen abundance in the sample lies in the range $12+$ $\log \mathrm{O} / \mathrm{H}=7.2-8.4$. The abundance ratios of the $\alpha$-elements to oxygen follow the trends found in our previous studies of low-metallicity emission-line galaxies. In particular, the new data confirm with a larger sample of $230 \mathrm{H}$ II regions the finding by Izotov et al. (2006) that $\mathrm{Ne} / \mathrm{O}$ increases with increasing oxygen abundance, which is interpreted as caused by a higher depletion of oxygen in higher-metallicity galaxies. The $\mathrm{S} / \mathrm{O}, \mathrm{Cl} / \mathrm{O}$ and $\mathrm{Ar} / \mathrm{O}$ abundance ratios are close to the distributions obtained previously by Izotov et al. (2006). Slight trends seen in $\mathrm{S} / \mathrm{O}, \mathrm{Cl} / \mathrm{O}$ and $\mathrm{Ar} / \mathrm{O}$ abundance ratios could imply a metallicity dependence of the $\alpha$-element production by massive stars. Variations of the explosion energy of Type II supernovae with metallicity might also play a role (Kobayashi et al. 2006). However, as already pointed out by Izotov et al. (2006), the abundance determinations of these elements may suffer from uncertainties owing to inaccurate ionization correction factors.

2. The Fe/O ratio shows an underabundance of iron relative to oxygen as compared to the solar value, which is particularly large for the high-metallicity galaxies. This again confirms the finding by Izotov et al. (2006) and strengthens our interpretation that $\mathrm{Fe}$ is depleted onto dust grains and that this effect depends on the metallicity.

3. There is a tendency for N/O to increase with decreasing oxygen abundance in extremely low-metallicity galaxies with $12+\log \mathrm{O} / \mathrm{H}<7.5$. This could be a sign of enhanced production of primary nitrogen by rapidly rotating metal-free stars (Meynet \& Maeder 2002).

4. The electron temperature derived from [S III] $\lambda 6312 / \lambda 9069$

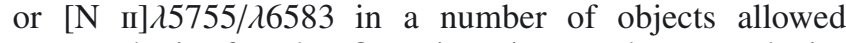
us to obtain for the first time in metal-poor galaxies an empirical relation between those temperatures and the one derived from [O $\mathrm{OII}] \lambda 4363 / \lambda(4959+5007)$. We also present the empirical relation between $t_{\mathrm{e}}$ derived from $\left[\begin{array}{ll}\mathrm{O} & \text { II }\end{array}\right] \lambda 3727 /(\lambda 7320+\lambda 7330)$ or $[\mathrm{S}$ II $] \lambda 4068 /(\lambda 6717+$ $\lambda 6730)$ and $[\mathrm{O} \mathrm{III}] \lambda 4363 / \lambda(4959+5007)$.

5. The electron number densities $N_{\mathrm{e}}(\mathrm{O}$ II $), N_{\mathrm{e}}(\mathrm{Cl}$ III $), N_{\mathrm{e}}(\mathrm{Ar}$ IV $)$ could be obtained for a number of objects in addition to $N_{\mathrm{e}}(\mathrm{S}$ II $)$. We find that $N_{\mathrm{e}}(\mathrm{O}$ II $)$ is very similar to $N_{\mathrm{e}}(\mathrm{S}$ II $)$, while $N_{\mathrm{e}}\left(\mathrm{Cl}\right.$ III) and $N_{\mathrm{e}}(\mathrm{Ar}$ IV) are systematically higher. This has potential implications when deriving the pregalactic helium abundance, since the He I lines are predominantly emitted in those higher density zones.

6. In a number of objects, the abundances of $\mathrm{C}^{++}$and $\mathrm{O}^{++}$could be derived from recombination lines (RLs). We find that $\mathrm{O}^{++}$ abundances obtained from RLs tend to be higher than those derived from collisionally excited lines (CELs), as found in previous studies of galactic and a few extragalactic $\mathrm{H}_{\text {II }}$ regions. In the $\mathrm{C}^{++} / \mathrm{O}^{++}$vs. $\mathrm{O} / \mathrm{H}$ diagram, most of the new points follow the relation obtained by previous observations of $\mathrm{C} / \mathrm{O}$ increasing with $\mathrm{O} / \mathrm{H}$.

Acknowledgements. N.G.G. and Y.I.I., K.J.F. are grateful to the staff of the MaxPlanck-Institute für Radioastronomie (Bonn) for their hospitality and acknowledge support through DFG grant No. FR 325/59-1. Y.I.I. thanks the Observatoire de Paris for hospitality and financial support. P.P. has been supported by a Ciencia 2008 contract, funded by FCT/MCTES (Portugal) and POPH/FSE (EC), and by the Wenner-Gren Foundation.

\section{References}

Asplund, M., Grevesse, N., Sauval, A. J., \& Scott, P. 2009, ARA\&A, 47, 481 Bohlin, R. C. 1996, AJ, 111, 1743

Campbell, A., Terlevich, R., \& Melnick, J. 1986, MNRAS, 223, 811

Colina, L., \& Bohlin, R. C. 1994, AJ, 108, 1931

Davey, A. R., Storey, P. J., \& Kisielius, R. 2000, A\&AS, 142, 85

Esteban, C., Bresolin, F., Peimbert, M., et al. 2009, ApJ, 700, 654 
García-Rojas, J., \& Esteban, C. 2007, ApJ, 670, 457

Garnett, D. R. 1992, AJ, 103, 1330

Garnett, D. R., Skillman, E. D., Dufour, R. J., et al. 1995, ApJ, 443, 64

Garnett, D. R., Shields, G. A., Peimbert, M., et al. 1999, ApJ, 513, 168

Guseva, N. G., Izotov, Y. I., \& Thuan, T. X. 2006, ApJ, 644, 890

Guseva, N. G., Izotov, Y. I., Papaderos, P., \& Fricke, K. J. 2007, A\&A, 464, 885

Guseva, N. G., Papaderos, P., Meyer, H. T., Izotov, Y. I., \& Fricke, K. J. 2009, A\&A, 505, 63

Izotov, Y. I., \& Thuan, T. X. 1999, ApJ, 511, 639

Izotov, Y. I., \& Thuan, T. X. 2004, ApJ, 602, 200

Izotov, Y. I., Stasińska, G., Guseva, N. G., \& Thuan, T. X. 2004, A\&A, 415, 87

Izotov, Y. I., Stasińska, G., Meynet, G., Guseva, N. G., \& Thuan, T. X. 2006, A\&A, 448, 955

Izotov, Y. I., Guseva, N. G., Fricke, K. J., \& Papaderos, P. 2009, A\&A, 503, 61

Izotov, Y. I., Guseva, N. G., \& Thuan, T. X. 2011, ApJ, 728, 161

Kehrig, C., Vílchez, J. M., Telles, E., Cuisinier, F., \& Pérez-Montero, E. 2006, A\&A, 457, 477

Kobayashi, C., Umeda, H., Nomoto, K., Tominaga, N., \& Ohkubo, T. 2006, ApJ, 653, 1145

Kobulnicky, H. A., \& Skillman, E. D. 1998, ApJ, 497, 601

Kunth, D., \& Sargent, W. L. W. 1983, ApJ, 273, 81
Lodders, K. 2003, ApJ, 591, 1220

Masegosa, J., Moles, M., \& Campos-Aguilar, A. 1994, ApJ, 420, 576

Melbourne, J., \& Salzer, J. J. 2002, AJ, 123, 2302

Meynet, G., \& Maeder, A. 2002, A\&A, 390, 561

Oke, J. B. 1990, AJ, 99, 1621

Papaderos, P., Guseva, N. G., Izotov, Y. I., et al. 2006, A\&A, 457, 45

Papaderos, P., Guseva, N. G., Izotov, Y. I., \& Fricke, K. J. 2008, A\&A, 491, 113 Peimbert, A., Peimbert, M., \& Ruiz, M. T. 2005, ApJ, 634, 1056

Pérez-Montero, E., \& Díaz, A. I. 2003, MNRAS, 346, 105

Pérez-Montero, E., \& Díaz, A. I. 2005, MNRAS, 361, 1063

Press, W. H., Teukolsky, S. A., Vetterling, W. T., \& Flannery, B. P. 1992,

Numerical recipes in FORTRAN, The art of scientific computing, 2nd edn. (Cambridge: University Press)

Smith, L. J., Norris, R. P. F., \& Crowther, P. A. 2002, MNRAS, 337, 1309

Stasińska, G. 2005, A\&A, 434, 507

Storey, P. J. 1994, A\&A, 282, 999

Terlevich, R., Melnick, J., Masegosa, J., Moles, M., \& Copetti, M. V. F. 1991, A\&AS, 91, 285

Thuan, T. X., \& Izotov, Y. I. 2005, ApJS, 161, 240

Thuan, T. X., Izotov, Y. I., \& Lipovetsky, V. A. 1995, ApJ, 445, 108

van Zee, L., \& Haynes, M. P. 2006, ApJ, 636, 214 
Table 2. Spectroscopic properties of $\mathrm{H}$ II regions.

\begin{tabular}{|c|c|c|c|c|c|c|}
\hline H II Region & $t_{\mathrm{e}}(\mathrm{O} \text { III })^{a}$ & $12+\log \mathrm{O} / \mathrm{H}$ & $E W(\mathrm{H} \alpha)^{b}$ & $F(\mathrm{H} \beta)^{c}$ & $C(\mathrm{H} \beta) \operatorname{dex}$ & $100 \times F\left(\left[\mathrm{O}_{\mathrm{III}}\right] 4363\right) / F(\mathrm{H} \beta)^{d}$ \\
\hline UM $254^{e}$ & $1.07 \pm 0.03$ & $8.16 \pm 0.03$ & $402 \pm 0$ & $356.7 \pm 0.5$ & $0.130 \pm 0.018$ & $1.7 \pm 0.1$ \\
\hline $\mathrm{UM} 283 \mathrm{D}^{e}$ & $1.24 \pm 0.03$ & $8.07 \pm 0.03$ & $314 \pm 0$ & $107.3 \pm 0.7$ & $0.225 \pm 0.020$ & $3.8 \pm 0.3$ \\
\hline $\mathrm{UM} 283 \mathrm{O}^{e}$ & $1.18 \pm 0.03$ & $8.06 \pm 0.03$ & $301 \pm 0$ & $47.9 \pm 0.1$ & $0.290 \pm 0.019$ & $3.2 \pm 0.2$ \\
\hline NGC 346 A No. $1^{g}$ & $1.23 \pm 0.01$ & $8.07 \pm 0.01$ & $1157 \pm 1$ & $602.5 \pm 0.6$ & $0.060 \pm 0.018$ & $6.5 \pm 0.1$ \\
\hline NGC 346 A No. $2^{g}$ & $1.27 \pm 0.01$ & $8.03 \pm 0.01$ & $1330 \pm 1$ & $414.4 \pm 0.5$ & $0.105 \pm 0.018$ & $6.6 \pm 0.1$ \\
\hline NGC 346 A No. $3^{g}$ & $1.24 \pm 0.01$ & $8.04 \pm 0.01$ & $392 \pm 0$ & $339.8 \pm 0.6$ & $0.065 \pm 0.018$ & $6.0 \pm 0.2$ \\
\hline NGC 346 B No. $1^{g}$ & $1.24 \pm 0.01$ & $8.05 \pm 0.01$ & $1121 \pm 1$ & $470.5 \pm 0.8$ & $0.060 \pm 0.019$ & $5.8 \pm 0.1$ \\
\hline NGC 346 B No. $2^{g}$ & $1.28 \pm 0.01$ & $8.01 \pm 0.01$ & $819 \pm 1$ & $534.3 \pm 0.8$ & $0.075 \pm 0.018$ & $6.7 \pm 0.1$ \\
\hline NGC 346 D No. $1^{g}$ & $1.32 \pm 0.01$ & $7.98 \pm 0.01$ & $1240 \pm 1$ & $531.4 \pm 0.8$ & $0.080 \pm 0.018$ & $6.7 \pm 0.1$ \\
\hline NGC 346 D No. $2^{g}$ & $1.33 \pm 0.01$ & $7.95 \pm 0.01$ & $1177 \pm 1$ & $350.2 \pm 0.6$ & $0.055 \pm 0.018$ & $7.5 \pm 0.2$ \\
\hline NGC 346 D No. $3^{g}$ & $1.27 \pm 0.01$ & $7.99 \pm 0.01$ & $1335 \pm 1$ & $403.3 \pm 0.6$ & $0.025 \pm 0.018$ & $6.3 \pm 0.1$ \\
\hline NGC 346 D No. $4^{g}$ & $1.30 \pm 0.01$ & $7.96 \pm 0.01$ & $1163 \pm 1$ & $404.7 \pm 0.6$ & $0.005 \pm 0.018$ & $6.7 \pm 0.1$ \\
\hline NGC 346 A No. $1^{f}$ & $1.30 \pm 0.01$ & $8.01 \pm 0.01$ & $1210 \pm 1$ & $517.0 \pm 0.4$ & $0.165 \pm 0.018$ & $7.1 \pm 0.1$ \\
\hline NGC 346 A No. $2^{f}$ & $1.26 \pm 0.01$ & $8.04 \pm 0.01$ & $1428 \pm 1$ & $381.3 \pm 0.3$ & $0.070 \pm 0.018$ & $6.4 \pm 0.1$ \\
\hline NGC 346 A No. $3^{f}$ & $1.27 \pm 0.01$ & $8.02 \pm 0.01$ & $556 \pm 0$ & $301.1 \pm 0.3$ & $0.090 \pm 0.018$ & $6.1 \pm 0.1$ \\
\hline NGC 346 B No. $1^{f}$ & $1.26 \pm 0.01$ & $8.05 \pm 0.01$ & $1153 \pm 1$ & $456.3 \pm 0.4$ & $0.165 \pm 0.018$ & $5.9 \pm 0.1$ \\
\hline NGC 346 B No. $2^{f}$ & $1.26 \pm 0.01$ & $8.02 \pm 0.01$ & $824 \pm 0$ & $557.6 \pm 0.5$ & $0.070 \pm 0.018$ & $6.2 \pm 0.1$ \\
\hline NGC 346 D No. $1^{f}$ & $1.29 \pm 0.01$ & $8.03 \pm 0.01$ & $1334 \pm 1$ & $536.0 \pm 0.4$ & $0.115 \pm 0.018$ & $6.2 \pm 0.1$ \\
\hline NGC 346 D No. $2^{f}$ & $1.25 \pm 0.01$ & $8.03 \pm 0.01$ & $1315 \pm 1$ & $359.3 \pm 0.3$ & $0.060 \pm 0.018$ & $6.4 \pm 0.1$ \\
\hline NGC 346 D No. $3^{f}$ & $1.28 \pm 0.01$ & $8.00 \pm 0.01$ & $1427 \pm 1$ & $392.6 \pm 0.3$ & $0.070 \pm 0.018$ & $6.3 \pm 0.1$ \\
\hline NGC 346 D No. $4^{f}$ & $1.30 \pm 0.01$ & $8.00 \pm 0.01$ & $1236 \pm 1$ & $405.8 \pm 0.4$ & $0.100 \pm 0.018$ & $6.6 \pm 0.1$ \\
\hline NGC 456 A No. $1^{g}$ & $1.23 \pm 0.01$ & $8.06 \pm 0.01$ & $2790 \pm 2$ & $371.9 \pm 0.7$ & $0.190 \pm 0.019$ & $4.3 \pm 0.1$ \\
\hline NGC 456 A No. $2^{g}$ & $1.23 \pm 0.01$ & $8.04 \pm 0.01$ & $1493 \pm 2$ & $214.7 \pm 0.5$ & $0.155 \pm 0.019$ & $4.8 \pm 0.1$ \\
\hline NGC 456 A No. $3^{g}$ & $1.21 \pm 0.01$ & $8.05 \pm 0.01$ & $2369 \pm 2$ & $246.6 \pm 0.5$ & $0.160 \pm 0.019$ & $4.4 \pm 0.1$ \\
\hline NGC 456 A No. $1^{f}$ & $1.23 \pm 0.01$ & $8.06 \pm 0.01$ & $2830 \pm 1$ & $377.6 \pm 0.3$ & $0.190 \pm 0.018$ & $4.2 \pm 0.1$ \\
\hline NGC 456 A No. $2^{f}$ & $1.20 \pm 0.01$ & $8.05 \pm 0.01$ & $1418 \pm 1$ & $231.7 \pm 0.3$ & $0.000 \pm 0.018$ & $4.8 \pm 0.1$ \\
\hline NGC 456 A No. $3^{f}$ & $1.21 \pm 0.01$ & $8.05 \pm 0.01$ & $2394 \pm 1$ & $255.5 \pm 0.3$ & $0.155 \pm 0.018$ & $4.3 \pm 0.1$ \\
\hline UM $133 \mathrm{H}^{e}$ & $1.55 \pm 0.02$ & $7.69 \pm 0.02$ & $926 \pm 1$ & $108.1 \pm 0.3$ & $0.125 \pm 0.019$ & $7.5 \pm 0.2$ \\
\hline $\mathrm{UM} 133 \mathrm{O}^{e}$ & $1.54 \pm 0.02$ & $7.71 \pm 0.01$ & $1070 \pm 1$ & $98.2 \pm 0.1$ & $0.195 \pm 0.018$ & $7.9 \pm 0.2$ \\
\hline $\mathrm{UM} 382^{e}$ & $1.56 \pm 0.01$ & $7.81 \pm 0.01$ & $1163 \pm 0$ & $50.3 \pm 0.0$ & $0.100 \pm 0.018$ & $12.4 \pm 0.2$ \\
\hline $\mathrm{UM} 408^{e}$ & $1.37 \pm 0.01$ & $7.94 \pm 0.01$ & $432 \pm 1$ & $49.4 \pm 0.1$ & $0.110 \pm 0.019$ & $8.0 \pm 0.2$ \\
\hline $\mathrm{UM} 417^{e}$ & $1.71 \pm 0.02$ & $7.67 \pm 0.01$ & $1748 \pm 1$ & $78.2 \pm 0.1$ & $0.110 \pm 0.018$ & $12.6 \pm 0.2$ \\
\hline UM 420 No. $2^{g}$ & $1.23 \pm 0.04$ & $8.16 \pm 0.04$ & $619 \pm 3$ & $14.0 \pm 0.1$ & $0.350 \pm 0.023$ & $5.9 \pm 0.6$ \\
\hline UM 420 No. $1^{f}$ & $1.21 \pm 0.04$ & $8.15 \pm 0.03$ & $167 \pm 1$ & $12.1 \pm 0.1$ & $0.000 \pm 0.020$ & $4.4 \pm 0.4$ \\
\hline UM 420 No. $2^{f}$ & $1.37 \pm 0.03$ & $8.04 \pm 0.02$ & $567 \pm 2$ & $10.5 \pm 0.1$ & $0.560 \pm 0.020$ & $6.6 \pm 0.3$ \\
\hline MRK $600^{e}$ & $1.50 \pm 0.02$ & $7.88 \pm 0.01$ & $555 \pm 0$ & $113.1 \pm 0.1$ & $0.225 \pm 0.018$ & $12.3 \pm 0.2$ \\
\hline CAM 0357-3915 & $1.54 \pm 0.02$ & $7.90 \pm 0.01$ & $992 \pm 2$ & $74.4 \pm 0.3$ & $0.235 \pm 0.019$ & $12.8 \pm 0.3$ \\
\hline CAM $0357-3915^{f}$ & $1.51 \pm 0.01$ & $7.90 \pm 0.01$ & $986 \pm 1$ & $46.9 \pm 0.1$ & $0.250 \pm 0.019$ & $11.8 \pm 0.2$ \\
\hline TOL $0513-393^{g}$ & $1.52 \pm 0.01$ & $7.94 \pm 0.01$ & $1722 \pm 2$ & $68.8 \pm 0.1$ & $0.365 \pm 0.019$ & $13.5 \pm 0.2$ \\
\hline TOL $0513-393^{f}$ & $1.51 \pm 0.01$ & $7.95 \pm 0.01$ & $1690 \pm 2$ & $77.0 \pm 0.1$ & $0.060 \pm 0.019$ & $14.8 \pm 0.2$ \\
\hline TOL $0618-402^{e}$ & $1.14 \pm 0.02$ & $8.20 \pm 0.02$ & $333 \pm 0$ & $37.4 \pm 0.1$ & $0.295 \pm 0.019$ & $4.3 \pm 0.2$ \\
\hline HE $2-10 C^{e}$ & $1.24 \pm 0.02$ & $7.87 \pm 0.02$ & $196 \pm 0$ & $2075.0 \pm 1.6$ & $0.495 \pm 0.018$ & $1.7 \pm 0.1$ \\
\hline $\mathrm{HE} 2-10 \mathrm{E}^{e}$ & $0.75 \pm 0.04$ & $8.40 \pm 0.06$ & $272 \pm 0$ & $3627.0 \pm 3.0$ & $0.670 \pm 0.018$ & $0.2 \pm 0.0$ \\
\hline NGC $3125^{e}$ & $1.11 \pm 0.01$ & $8.26 \pm 0.01$ & $867 \pm 0$ & $863.3 \pm 0.5$ & $0.390 \pm 0.018$ & $4.6 \pm 0.1$ \\
\hline MRK $1259^{e}$ & $1.01 \pm 0.03$ & $8.12 \pm 0.04$ & $247 \pm 0$ & $1025.0 \pm 1.2$ & $0.660 \pm 0.018$ & $0.9 \pm 0.1$ \\
\hline MRK $1271^{e}$ & $1.34 \pm 0.01$ & $7.99 \pm 0.01$ & $604 \pm 0$ & $1368.0 \pm 0.9$ & $0.190 \pm 0.018$ & $8.0 \pm 0.1$ \\
\hline $\mathrm{POX} 4^{e}$ & $1.31 \pm 0.01$ & $8.07 \pm 0.01$ & $1223 \pm 0$ & $1457.0 \pm 0.8$ & $0.170 \pm 0.018$ & $8.8 \pm 0.1$ \\
\hline TOL $1214-277^{e}$ & $2.00 \pm 0.02$ & $7.53 \pm 0.01$ & $1644 \pm 1$ & $700.8 \pm 0.5$ & $0.285 \pm 0.018$ & $15.9 \pm 0.2$ \\
\hline TOL $1214-277^{f}$ & $1.97 \pm 0.02$ & $7.55 \pm 0.01$ & $1819 \pm 2$ & $111.8 \pm 0.2$ & $0.250 \pm 0.018$ & $16.1 \pm 0.2$ \\
\hline TOL 65 No. $1^{f}$ & $1.78 \pm 0.02$ & $7.53 \pm 0.01$ & $1684 \pm 1$ & $150.8 \pm 0.2$ & $0.285 \pm 0.018$ & $9.6 \pm 0.2$ \\
\hline TOL 65 No. $2^{f}$ & $1.76 \pm 0.03$ & $7.56 \pm 0.01$ & $441 \pm 1$ & $35.6 \pm 0.1$ & $0.180 \pm 0.019$ & $8.7 \pm 0.2$ \\
\hline $\mathrm{J} 1253-0312^{e}$ & $1.39 \pm 0.01$ & $8.00 \pm 0.01$ & $1395 \pm 0$ & $1834.0 \pm 0.8$ & $0.265 \pm 0.018$ & $9.6 \pm 0.1$ \\
\hline NGC $5253 \mathrm{No.C1}^{e}$ & $1.23 \pm 0.01$ & $8.14 \pm 0.01$ & $2734 \pm 0$ & $2559.0 \pm 1.6$ & $0.380 \pm 0.018$ & $6.7 \pm 0.1$ \\
\hline NGC 5253 No.C $2^{e}$ & $1.03 \pm 0.01$ & $8.31 \pm 0.01$ & $802 \pm 0$ & $1019.0 \pm 1.0$ & $0.125 \pm 0.018$ & $3.0 \pm 0.1$ \\
\hline NGC 5253 No.P1 $^{e}$ & $1.35 \pm 0.01$ & $8.00 \pm 0.01$ & $761 \pm 0$ & $1954.0 \pm 2.0$ & $0.180 \pm 0.018$ & $\ldots$ \\
\hline NGC 5253 No.P2 ${ }^{e}$ & $1.24 \pm 0.01$ & $8.15 \pm 0.01$ & $3598 \pm 2$ & $3265.0 \pm 2.6$ & $0.335 \pm 0.018$ & $7.2 \pm 0.1$ \\
\hline TOL 89 No. $1^{e}$ & $1.03 \pm 0.01$ & $8.34 \pm 0.01$ & $1565 \pm 1$ & $341.9 \pm 0.3$ & $0.330 \pm 0.018$ & $3.1 \pm 0.1$ \\
\hline TOL 89 No. $2^{e}$ & $0.94 \pm 0.02$ & $8.40 \pm 0.02$ & $306 \pm 0$ & $174.8 \pm 0.3$ & $0.105 \pm 0.018$ & $2.0 \pm 0.1$ \\
\hline NGC 5408 No. $1^{e}$ & $1.56 \pm 0.01$ & $8.03 \pm 0.01$ & $1491 \pm 0$ & $1194.0 \pm 0.8$ & $0.520 \pm 0.018$ & $17.0 \pm 0.2$ \\
\hline NGC 5408 No. $2^{e}$ & $1.37 \pm 0.01$ & $8.13 \pm 0.01$ & $2541 \pm 1$ & $404.4 \pm 0.4$ & $0.355 \pm 0.018$ & $10.8 \pm 0.2$ \\
\hline TOL $1457-262^{e}$ & $1.19 \pm 0.01$ & $8.27 \pm 0.01$ & $644 \pm 0$ & $733.0 \pm 0.7$ & $0.745 \pm 0.018$ & $5.5 \pm 0.1$ \\
\hline TOL $1924-416.1^{e}$ & $1.25 \pm 0.01$ & $7.99 \pm 0.01$ & $381 \pm 0$ & $988.6 \pm 1.1$ & $0.005 \pm 0.018$ & $5.7 \pm 0.1$ \\
\hline TOL $1924-416.2^{e}$ & $1.23 \pm 0.01$ & $8.09 \pm 0.01$ & $884 \pm 0$ & $961.0 \pm 0.8$ & $0.090 \pm 0.018$ & $6.2 \pm 0.1$ \\
\hline NGC $6822 \mathrm{~V}^{e}$ & $1.14 \pm 0.01$ & $8.16 \pm 0.01$ & $1416 \pm 0$ & $1643.0 \pm 0.7$ & $0.470 \pm 0.018$ & $4.6 \pm 0.1$ \\
\hline NGC $6822 \mathrm{~V}$ No. $1^{g}$ & $1.17 \pm 0.01$ & $8.15 \pm 0.01$ & $1152 \pm 1$ & $94.6 \pm 0.2$ & $0.590 \pm 0.019$ & $4.9 \pm 0.2$ \\
\hline
\end{tabular}


Table 2. continued.

\begin{tabular}{|c|c|c|c|c|c|c|}
\hline H II Region & $t_{\mathrm{e}}(\mathrm{O} \text { III })^{a}$ & $12+\log \mathrm{O} / \mathrm{H}$ & $E W(\mathrm{H} \alpha)^{b}$ & $F(\mathrm{H} \beta)^{c}$ & $C(\mathrm{H} \beta) \operatorname{dex}$ & $100 \times F\left(\left[\mathrm{O}_{\mathrm{III}}\right] 4363\right) / F(\mathrm{H} \beta)^{d}$ \\
\hline NGC $6822 \mathrm{~V}$ No. $2^{g}$ & $1.21 \pm 0.02$ & $8.13 \pm 0.02$ & $1079 \pm 2$ & $38.5 \pm 0.2$ & $0.605 \pm 0.019$ & $5.6 \pm 0.2$ \\
\hline NGC $6822 \mathrm{~V}$ No. $1^{f}$ & $1.19 \pm 0.01$ & $8.13 \pm 0.01$ & $1090 \pm 1$ & $66.2 \pm 0.1$ & $0.545 \pm 0.019$ & $5.4 \pm 0.1$ \\
\hline NGC $6822 \mathrm{~V}$ No. $2^{f}$ & $1.22 \pm 0.02$ & $8.10 \pm 0.01$ & $1066 \pm 2$ & $24.1 \pm 0.1$ & $0.590 \pm 0.019$ & $5.6 \pm 0.2$ \\
\hline NGC $6822 X^{f}$ & $1.24 \pm 0.04$ & $8.03 \pm 0.03$ & $335 \pm 1$ & $7.6 \pm 0.1$ & $0.255 \pm 0.021$ & $5.4 \pm 0.4$ \\
\hline TOL 2138-405 No. $1^{g}$ & $1.31 \pm 0.02$ & $8.12 \pm 0.01$ & $1605 \pm 3$ & $34.5 \pm 0.1$ & $0.305 \pm 0.019$ & $8.9 \pm 0.2$ \\
\hline TOL 2138-405 No. $1^{f}$ & $1.39 \pm 0.01$ & $8.02 \pm 0.01$ & $1488 \pm 2$ & $58.1 \pm 0.1$ & $0.290 \pm 0.019$ & $9.7 \pm 0.2$ \\
\hline TOL 2138-405 No. $3^{f}$ & $1.45 \pm 0.02$ & $7.96 \pm 0.01$ & $1084 \pm 3$ & $11.8 \pm 0.1$ & $0.265 \pm 0.020$ & $9.2 \pm 0.3$ \\
\hline TOL 2146-391 No. $1^{g}$ & $1.60 \pm 0.02$ & $7.82 \pm 0.01$ & $1815 \pm 4$ & $67.0 \pm 0.3$ & $0.315 \pm 0.019$ & $12.2 \pm 0.3$ \\
\hline TOL 2146-391 No. $2^{g}$ & $1.65 \pm 0.02$ & $7.77 \pm 0.01$ & $1489 \pm 3$ & $50.6 \pm 0.2$ & $0.305 \pm 0.020$ & $12.6 \pm 0.3$ \\
\hline TOL 2146-391 No. ${ }^{f}$ & $1.58 \pm 0.02$ & $7.82 \pm 0.01$ & $1734 \pm 2$ & $77.9 \pm 0.1$ & $0.320 \pm 0.019$ & $11.7 \pm 0.2$ \\
\hline TOL 2146-391 No. $2^{f}$ & $1.61 \pm 0.02$ & $7.79 \pm 0.01$ & $1319 \pm 2$ & $54.5 \pm 0.1$ & $0.305 \pm 0.019$ & $11.7 \pm 0.2$ \\
\hline TOL $2240-384^{g}$ & $1.58 \pm 0.02$ & $7.85 \pm 0.01$ & $1214 \pm 2$ & $72.8 \pm 0.3$ & $0.815 \pm 0.019$ & $11.2 \pm 0.2$ \\
\hline TOL $2240-384^{f}$ & $1.45 \pm 0.01$ & $7.93 \pm 0.01$ & $1191 \pm 1$ & $80.0 \pm 0.2$ & $0.830 \pm 0.019$ & $9.6 \pm 0.2$ \\
\hline PHL 293B ${ }^{e}$ & $1.69 \pm 0.02$ & $7.72 \pm 0.01$ & $53 \pm 0$ & $71.4 \pm 0.1$ & $0.080 \pm 0.018$ & $14.2 \pm 0.3$ \\
\hline UM 160 No. $1^{g}$ & $1.36 \pm 0.03$ & $7.97 \pm 0.02$ & $1407 \pm 4$ & $27.4 \pm 0.2$ & $0.470 \pm 0.021$ & $7.7 \pm 0.4$ \\
\hline UM 160 No. $1^{f}$ & $1.41 \pm 0.02$ & $7.94 \pm 0.01$ & $1443 \pm 2$ & $33.0 \pm 0.1$ & $0.460 \pm 0.019$ & $8.1 \pm 0.2$ \\
\hline UM 160 No. $2^{f}$ & $1.24 \pm 0.02$ & $8.09 \pm 0.02$ & $399 \pm 1$ & $11.5 \pm 0.1$ & $0.000 \pm 0.020$ & $6.3 \pm 0.3$ \\
\hline UM 160 No. $3^{f}$ & $1.17 \pm 0.01$ & $8.10 \pm 0.01$ & $882 \pm 2$ & $12.1 \pm 0.1$ & $0.315 \pm 0.020$ & $4.0 \pm 0.1$ \\
\hline
\end{tabular}

Notes. ${ }^{(a)} t_{\mathrm{e}}=10^{-4} T_{\mathrm{e}}$; ${ }^{(b)}$ in $\AA$; ${ }^{(c)}$ in units $10^{-16} \mathrm{erg} \mathrm{s}^{-1} \mathrm{~cm}^{-2}$; ${ }^{(d)}$ fluxes non-corrected for extinction; ${ }^{(e)}$ UVES data; ${ }^{(f)}$ medium resolution FORS data; ${ }^{(g)}$ low resolution FORS data.

Table 3. Extinction-corrected emission line fluxes (UVES observations).

\begin{tabular}{|c|c|c|c|c|}
\hline \multirow{3}{*}{ Line } & \multicolumn{4}{|c|}{$\begin{array}{c}\text { Galaxy } \\
\text { (ESO ProGRAM) }\end{array}$} \\
\hline & UM 254 & UM 283D & UM 2830 & $\overline{\mathrm{UM}} 133 \mathrm{H}$ \\
\hline & $(71 . \mathrm{B}-0055(\mathrm{~A}))$ & (71.B-0055(A)) & $(70 . \mathrm{B}-0717(\mathrm{~A}))$ & $(68 . \mathrm{B}-0310(\mathrm{~A}))$ \\
\hline 3727 [O II] & $250.92 \pm 3.88$ & $294.65 \pm 5.03$ & $236.44 \pm 3.76$ & $107.18 \pm 1.70$ \\
\hline $3750 \mathrm{H} 12$ & $3.00 \pm 0.05$ & $3.58 \pm 0.87$ & $5.29 \pm 0.67$ & $3.95 \pm 0.29$ \\
\hline 3771 H11 & $3.20 \pm 0.19$ & $4.78 \pm 0.49$ & $6.20 \pm 0.43$ & $5.08 \pm 0.29$ \\
\hline 3798 H10 & $5.55 \pm 0.29$ & $6.11 \pm 0.53$ & $8.14 \pm 0.39$ & $5.54 \pm 0.30$ \\
\hline $3820 \mathrm{He}_{\mathrm{I}}$ & $0.86 \pm 0.25$ & & & \\
\hline 3835 H9 & $6.30 \pm 0.20$ & $8.63 \pm 0.60$ & $9.45 \pm 0.39$ & $7.69 \pm 0.31$ \\
\hline 3868 [Ne III] & $22.21 \pm 0.41$ & $27.38 \pm 0.57$ & $24.78 \pm 0.49$ & $28.26 \pm 0.69$ \\
\hline $3889 \mathrm{He} \mathrm{I}^{+} \mathrm{H} 8$ & $20.52 \pm 0.35$ & $20.48 \pm 0.64$ & $20.02 \pm 0.49$ & $20.52 \pm 0.52$ \\
\hline $4026 \mathrm{He} \mathrm{I}$ & $1.77 \pm 0.14$ & $1.99 \pm 0.26$ & $1.96 \pm 0.18$ & $\ldots$ \\
\hline 4068 [S II] & $1.97 \pm 0.10$ & $3.17 \pm 0.27$ & $2.36 \pm 0.18$ & \\
\hline $4101 \mathrm{H} \delta$ & $25.37 \pm 0.40$ & $27.96 \pm 0.67$ & $26.51 \pm 0.51$ & $26.79 \pm 0.57$ \\
\hline $4340 \mathrm{H} \gamma$ & $48.03 \pm 0.71$ & $48.22 \pm 0.90$ & $45.88 \pm 0.75$ & $47.09 \pm 0.80$ \\
\hline 4363 [O III] & $1.80 \pm 0.13$ & $4.10 \pm 0.29$ & $3.39 \pm 0.23$ & $7.72 \pm 0.24$ \\
\hline $4471 \mathrm{He}$ I & $3.66 \pm 0.12$ & $3.93 \pm 0.20$ & $\ldots$ & $3.70 \pm 0.14$ \\
\hline 4658 [Fe III] & $2.72 \pm 0.21$ & & . & \\
\hline $4861 \mathrm{H} \beta$ & $100.00 \pm 1.44$ & $100.00 \pm 1.75$ & $100.00 \pm 1.49$ & $100.00 \pm 1.50$ \\
\hline $4921 \mathrm{He} \mathrm{I}$ & $0.58 \pm 0.04$ & $\ldots$ & $0.97 \pm 0.05$ & \\
\hline 4959 [O III] & $75.15 \pm 1.08$ & $118.61 \pm 1.89$ & $107.90 \pm 1.60$ & $127.57 \pm 1.91$ \\
\hline 5007 [O III] & $231.11 \pm 3.31$ & $336.63 \pm 5.33$ & $324.58 \pm 4.79$ & $367.90 \pm 5.43$ \\
\hline $5015 \mathrm{He}$ I & $2.03 \pm 0.10$ & $2.38 \pm 0.12$ & $2.03 \pm 0.05$ & $1.93 \pm 0.08$ \\
\hline $5876 \mathrm{He}_{\mathrm{I}}$ & $11.18 \pm 0.23$ & $11.26 \pm 0.35$ & $10.60 \pm 0.20$ & $10.02 \pm 0.23$ \\
\hline $6300\left[\mathrm{O}_{\mathrm{I}}\right]$ & $5.35 \pm 0.12$ & $4.65 \pm 0.21$ & $4.66 \pm 0.12$ & $2.09 \pm 0.10$ \\
\hline 6312 [S III] & $1.01 \pm 0.08$ & $1.81 \pm 0.11$ & $1.61 \pm 0.07$ & $1.54 \pm 0.07$ \\
\hline 6363 [O I] & $1.63 \pm 0.08$ & $1.84 \pm 0.17$ & $1.80 \pm 0.09$ & $\ldots$ \\
\hline 6548 [N II] & $13.76 \pm 0.24$ & $6.63 \pm 0.18$ & $6.52 \pm 0.12$ & $1.20 \pm 0.06$ \\
\hline $6563 \mathrm{H} \alpha$ & $287.16 \pm 4.46$ & $284.50 \pm 4.90$ & $285.75 \pm 4.58$ & $279.30 \pm 4.48$ \\
\hline 6583 [N II] & $37.50 \pm 0.60$ & $19.97 \pm 0.40$ & $19.69 \pm 0.34$ & $3.13 \pm 0.10$ \\
\hline $6678 \mathrm{He}$ I & $3.53 \pm 0.16$ & $3.09 \pm 0.13$ & $2.94 \pm 0.07$ & $2.81 \pm 0.08$ \\
\hline 6717 [S II] & $\ldots$ & $29.30 \pm 0.58$ & $\ldots$ & $9.40 \pm 0.18$ \\
\hline $6731[\mathrm{~S}$ II] & $\ldots$ & $20.80 \pm 0.41$ & $\ldots$ & $6.94 \pm 0.13$ \\
\hline $7065 \mathrm{He}_{\mathrm{I}}$ & $2.15 \pm 0.06$ & $2.77 \pm 0.09$ & $\ldots$ & $2.29 \pm 0.06$ \\
\hline 7136 [Ar III] & $7.03 \pm 0.13$ & $9.35 \pm 0.19$ & $\ldots$ & $4.34 \pm 0.10$ \\
\hline $7281 \mathrm{He} \mathrm{I}$ & $\ldots$ & $\ldots$ & $\ldots$ & $0.54 \pm 0.05$ \\
\hline 7320 [O II] & $\ldots$ & $3.55 \pm 0.12$ & $\ldots$ & $1.74 \pm 0.06$ \\
\hline
\end{tabular}


Table 3. continued.

\begin{tabular}{|c|c|c|c|c|}
\hline \multirow[b]{2}{*}{ Line } & \multicolumn{4}{|c|}{$\begin{array}{c}\text { GALAXY } \\
\text { (ESO PROGRAM) }\end{array}$} \\
\hline & $\begin{array}{r}\text { UM 254 } \\
(71 . \mathrm{B}-0055(\mathrm{~A}))\end{array}$ & $\begin{array}{r}\text { UM 283D } \\
(71 . \mathrm{B}-0055(\mathrm{~A}))\end{array}$ & $\begin{array}{r}\text { UM 283O } \\
(70 . \mathrm{B}-0717(\mathrm{~A}))\end{array}$ & $\begin{array}{r}\text { UM 133H } \\
(68 . \mathrm{B}-0310(\mathrm{~A}))\end{array}$ \\
\hline $7330[\mathrm{O}$ II] & $\ldots$ & $3.04 \pm 0.11$ & $\ldots$ & $1.61 \pm 0.06$ \\
\hline $9069\left[\mathrm{~S}_{\mathrm{III}}\right]$ & $\ldots$ & $20.72 \pm 0.89$ & $\ldots$ & $7.42 \pm 0.24$ \\
\hline$C(\mathrm{H} \beta)$ & 0.130 & 0.225 & 0.290 & 0.125 \\
\hline$F(\mathrm{H} \beta)^{a}$ & 356.70 & 107.30 & 47.86 & 108.10 \\
\hline$E W(\mathrm{H} \beta) \AA$ & 85.2 & 91.1 & 73.7 & 200.5 \\
\hline$E W(\mathrm{abs}) \AA$ & 0.35 & 0.15 & 1.95 & 2.00 \\
\hline \multirow[b]{2}{*}{ Line } & \multicolumn{4}{|c|}{$\begin{array}{c}\text { GALAXY } \\
\text { (ESO PROGRAM) }\end{array}$} \\
\hline & $\begin{array}{r}\text { UM 1330 } \\
(70 . \mathrm{B}-0717(\mathrm{~A}))\end{array}$ & $\begin{array}{r}\text { UM382 } \\
(70 . \mathrm{B}-0717(\mathrm{~A}))\end{array}$ & $\begin{array}{r}\text { UM 408 } \\
(70 . \mathrm{B}-0717(\mathrm{~A}))\end{array}$ & $\begin{array}{r}\text { UM 417 } \\
(70 . \mathrm{B}-0717(\mathrm{~A}))\end{array}$ \\
\hline 3727 [O II] & $90.11 \pm 1.46$ & $44.56 \pm 0.70$ & $105.56 \pm 1.67$ & $61.53 \pm 0.99$ \\
\hline 3750 H12 & $3.58 \pm 0.19$ & $2.94 \pm 0.08$ & $4.83 \pm 0.31$ & $3.79 \pm 0.19$ \\
\hline 3771 H11 & $4.10 \pm 0.15$ & $3.71 \pm 0.10$ & $5.77 \pm 0.39$ & $5.11 \pm 0.18$ \\
\hline 3798 H10 & $5.63 \pm 0.20$ & $4.69 \pm 0.13$ & $6.87 \pm 0.44$ & $5.93 \pm 0.20$ \\
\hline $3820 \mathrm{He}_{\mathrm{I}}$ & $\ldots$ & $0.87 \pm 0.05$ & $\ldots$ & $0.75 \pm 0.06$ \\
\hline $3835 \mathrm{H} 9$ & $7.62 \pm 0.20$ & $6.71 \pm 0.13$ & $8.04 \pm 0.31$ & $7.78 \pm 0.23$ \\
\hline 3868 [Ne III] & $30.09 \pm 0.50$ & $43.19 \pm 0.66$ & $38.27 \pm 0.67$ & $39.29 \pm 0.65$ \\
\hline $3889 \mathrm{He}_{\mathrm{I}}+\mathrm{H} 8$ & $19.50 \pm 0.38$ & $17.70 \pm 0.30$ & $17.31 \pm 0.42$ & $21.13 \pm 0.38$ \\
\hline $3968[\mathrm{Ne} \mathrm{III}]+\mathrm{H} 7$ & $\ldots$ & $29.22 \pm 0.46$ & $28.46 \pm 0.58$ & $29.98 \pm 0.56$ \\
\hline $4026 \mathrm{He}_{\mathrm{I}}$ & $1.68 \pm 0.10$ & $1.69 \pm 0.09$ & $1.37 \pm 0.26$ & $1.79 \pm 0.08$ \\
\hline $4068[\mathrm{~S}$ II $]$ & $0.88 \pm 0.11$ & $0.43 \pm 0.07$ & $1.08 \pm 0.14$ & $0.62 \pm 0.08$ \\
\hline $4076\left[\mathrm{~S}_{\mathrm{II}}\right]$ & $0.30 \pm 0.07$ & $0.33 \pm 0.05$ & & 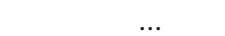 \\
\hline $4101 \mathrm{H} \delta$ & $26.13 \pm 0.43$ & $24.73 \pm 0.39$ & $25.67 \pm 0.56$ & $27.22 \pm 0.46$ \\
\hline $4340 \mathrm{H} \gamma$ & $47.33 \pm 0.72$ & $47.86 \pm 0.70$ & $47.13 \pm 0.77$ & $48.34 \pm 0.76$ \\
\hline 4363 [O III] & $8.33 \pm 0.18$ & $12.80 \pm 0.20$ & $8.09 \pm 0.19$ & $12.96 \pm 0.21$ \\
\hline $4387 \mathrm{He}$ I & $\ldots$ & $0.49 \pm 0.03$ & & $\ldots$ \\
\hline $4471 \mathrm{He}_{\mathrm{I}}$ & $3.41 \pm 0.09$ & $4.15 \pm 0.07$ & $3.58 \pm 0.11$ & $3.49 \pm 0.12$ \\
\hline $4861 \mathrm{H} \beta$ & $100.00 \pm 1.45$ & $100.00 \pm 1.42$ & $100.00 \pm 1.48$ & $100.00 \pm 1.44$ \\
\hline $4921 \mathrm{He}_{\mathrm{I}}$ & $1.00 \pm 0.03$ & $1.02 \pm 0.02$ & $0.95 \pm 0.04$ & $0.83 \pm 0.02$ \\
\hline 4959 [O III] & $138.76 \pm 2.00$ & $202.72 \pm 2.88$ & $172.21 \pm 2.53$ & $172.41 \pm 2.48$ \\
\hline 5007 [O III] & $402.31 \pm 5.79$ & $606.61 \pm 8.62$ & $522.89 \pm 7.67$ & $509.11 \pm 7.30$ \\
\hline $5015 \mathrm{He}_{\mathrm{I}}$ & $2.21 \pm 0.04$ & $1.96 \pm 0.03$ & $1.77 \pm 0.06$ & $1.85 \pm 0.03$ \\
\hline $5518[\mathrm{Cl} \mathrm{III}]$ & $\ldots$ & $0.35 \pm 0.02$ & $\ldots$ & $0.18 \pm 0.00$ \\
\hline $5538[\mathrm{Cl} \mathrm{III}]$ & $\ldots$ & $0.22 \pm 0.01$ & $\ldots$ & $\ldots$ \\
\hline $5876 \mathrm{He}_{\mathrm{I}}$ & $9.56 \pm 0.16$ & $10.17 \pm 0.15$ & $10.90 \pm 0.19$ & $9.96 \pm 0.16$ \\
\hline $6300\left[\mathrm{O}_{\mathrm{I}}\right]$ & $2.18 \pm 0.05$ & $1.09 \pm 0.02$ & $2.42 \pm 0.06$ & $1.37 \pm 0.03$ \\
\hline $6312[\mathrm{~S}$ III] & $1.52 \pm 0.04$ & $1.86 \pm 0.03$ & $1.62 \pm 0.05$ & $1.45 \pm 0.03$ \\
\hline $6363[\mathrm{O} \mathrm{I}]$ & $0.74 \pm 0.04$ & $0.45 \pm 0.01$ & $0.67 \pm 0.04$ & $0.52 \pm 0.03$ \\
\hline 6548 [N II] & $1.08 \pm 0.03$ & $0.75 \pm 0.02$ & $1.61 \pm 0.05$ & $0.72 \pm 0.04$ \\
\hline $6563 \mathrm{H} \alpha$ & $279.79 \pm 4.37$ & $279.00 \pm 4.30$ & $281.83 \pm 4.49$ & $277.19 \pm 4.32$ \\
\hline 6583 [N II] & $3.01 \pm 0.06$ & $2.52 \pm 0.04$ & $5.29 \pm 0.11$ & $2.46 \pm 0.05$ \\
\hline $6678 \mathrm{He} \mathrm{I}$ & $2.91 \pm 0.06$ & $3.02 \pm 0.06$ & $2.63 \pm 0.10$ & $2.69 \pm 0.06$ \\
\hline $6717\left[\mathrm{~S}_{\mathrm{II}}\right]$ & $9.27 \pm 0.17$ & $5.37 \pm 0.09$ & $11.48 \pm 0.20$ & $6.05 \pm 0.11$ \\
\hline $6731[\mathrm{~S}$ II] & $6.72 \pm 0.12$ & $\ldots$ & $7.52 \pm 0.14$ & $4.55 \pm 0.08$ \\
\hline$C(\mathrm{H} \beta)$ & 0.195 & 0.100 & 0.110 & 0.110 \\
\hline$F(\mathrm{H} \beta)^{a}$ & 98.23 & 50.30 & 49.36 & 78.19 \\
\hline$E W(\mathrm{H} \beta) \AA$ & 181.6 & 266.9 & 89.1 & 335.4 \\
\hline$E W($ abs $) \AA$ & 1.70 & 0.25 & 2.15 & 1.50 \\
\hline \multirow{3}{*}{ Line } & \multicolumn{4}{|c|}{$\begin{array}{c}\text { GALAXY } \\
\text { (ESO PROGRAM) }\end{array}$} \\
\hline & MRK 600 & TOL 0618-402 & HE 2-10C & HE 2-10E \\
\hline & $(70 . \mathrm{B}-0717(\mathrm{~A}))$ & (70.B-0717(A)) & $(073 . \mathrm{B}-0283(\mathrm{~A}))$ & (081.C-0113(A)) \\
\hline $3727[\mathrm{O} \mathrm{II]}$ & $38.57 \pm 0.69$ & $210.01 \pm 3.38$ & $211.87 \pm 3.33$ & $193.03 \pm 3.09$ \\
\hline $3750 \mathrm{H} 12$ & $6.54 \pm 0.36$ & $3.37 \pm 0.34$ & $3.44 \pm 0.24$ & $6.70 \pm 0.48$ \\
\hline 3771 H11 & $7.12 \pm 0.39$ & $3.93 \pm 0.35$ & $4.09 \pm 0.22$ & $6.55 \pm 1.01$ \\
\hline 3798 H10 & $8.31 \pm 0.45$ & $5.43 \pm 0.34$ & $5.33 \pm 0.19$ & $7.24 \pm 0.57$ \\
\hline $3820 \mathrm{He}_{\mathrm{I}}$ & $0.80 \pm 0.10$ & & $\cdots$ & $\ldots$ \\
\hline 3835 H9 & $9.52 \pm 0.31$ & $6.54 \pm 0.42$ & $6.41 \pm 0.18$ & $8.57 \pm 0.22$ \\
\hline 3868 [Ne III] & $37.83 \pm 0.64$ & $42.18 \pm 0.83$ & $7.50 \pm 0.16$ & $4.31 \pm 0.10$ \\
\hline $3889 \mathrm{He} \mathrm{I}+\mathrm{H} 8$ & $17.46 \pm 0.38$ & $20.33 \pm 0.47$ & $14.30 \pm 0.26$ & $16.67 \pm 0.30$ \\
\hline $3968[\mathrm{Ne}$ III $]+\mathrm{H} 7$ & $28.16 \pm 0.52$ & $27.45 \pm 0.73$ & $\ldots$ & $16.70 \pm 0.28$ \\
\hline $4026 \mathrm{He} \mathrm{I}$ & $1.77 \pm 0.14$ & $1.77 \pm 0.21$ & $\ldots$ & $0.36 \pm 0.07$ \\
\hline
\end{tabular}


Table 3. continued.

\begin{tabular}{|c|c|c|c|c|}
\hline \multirow[b]{2}{*}{ Line } & \multicolumn{4}{|c|}{$\begin{array}{c}\text { GALAXY } \\
\text { (ESO PROGRAM) }\end{array}$} \\
\hline & $\begin{array}{r}\text { MRK 600 } \\
(70 . \mathrm{B}-0717(\mathrm{~A}))\end{array}$ & $\begin{array}{r}\text { TOL 0618-402 } \\
(70 . \mathrm{B}-0717(\mathrm{~A}))\end{array}$ & $\begin{array}{r}\text { HE 2-10C } \\
(073 . \mathrm{B}-0283(\mathrm{~A}))\end{array}$ & $\begin{array}{r}\text { HE 2-10E } \\
(081 . \mathrm{C}-0113(\mathrm{~A}))\end{array}$ \\
\hline $4068[\mathrm{~S}$ II] & $\ldots$ & $1.97 \pm 0.18$ & $1.57 \pm 0.09$ & $1.00 \pm 0.04$ \\
\hline $4076[$ S II] & $\ldots$ & $\ldots$ & $\ldots$ & $0.21 \pm 0.02$ \\
\hline $4101 \mathrm{H} \delta$ & $25.69 \pm 0.48$ & $26.77 \pm 0.52$ & $24.88 \pm 0.41$ & $26.50 \pm 0.42$ \\
\hline $4340 \mathrm{H} \gamma$ & $45.95 \pm 0.73$ & $47.92 \pm 0.78$ & $47.84 \pm 0.71$ & $45.80 \pm 0.69$ \\
\hline 4363 [O III] & $12.51 \pm 0.24$ & $4.66 \pm 0.22$ & $1.96 \pm 0.09$ & $0.26 \pm 0.05$ \\
\hline $4387 \mathrm{He}_{\mathrm{I}}$ & $0.51 \pm 0.01$ & $\ldots$ & $\ldots$ & $\ldots$ \\
\hline $4471 \mathrm{He}_{\mathrm{I}}$ & $3.71 \pm 0.09$ & $\ldots$ & $2.54 \pm 0.07$ & $2.08 \pm 0.04$ \\
\hline 4658 [Fe III] & $\ldots$ & $1.13 \pm 0.06$ & $1.61 \pm 0.05$ & $1.88 \pm 0.05$ \\
\hline $4686 \mathrm{He}_{\text {II }}$ & $\ldots$ & $0.91 \pm 0.07$ & $\ldots$ & $0.66 \pm 0.03$ \\
\hline 4711 [Ar IV] + He I & $\ldots$ & $\ldots$ & $\ldots$ & $0.14 \pm 0.03$ \\
\hline $4861 \mathrm{H} \beta$ & $100.00 \pm 1.50$ & $100.00 \pm 1.49$ & $100.00 \pm 1.46$ & $100.00 \pm 1.48$ \\
\hline $4921 \mathrm{He}$ I & $1.01 \pm 0.03$ & $1.15 \pm 0.07$ & & $0.44 \pm 0.02$ \\
\hline 4959 [O III] & $216.05 \pm 3.23$ & $161.29 \pm 2.36$ & $55.28 \pm 0.81$ & $42.67 \pm 0.63$ \\
\hline 5007 [O III] & $648.02 \pm 9.67$ & $486.05 \pm 7.08$ & $159.52 \pm 2.32$ & $126.64 \pm 1.87$ \\
\hline $5015 \mathrm{He}_{\mathrm{I}}$ & $2.22 \pm 0.05$ & $2.18 \pm 0.08$ & $1.28 \pm 0.04$ & $0.87 \pm 0.03$ \\
\hline $5199[\mathrm{~N} \mathrm{I}]$ & $\ldots$ & $\ldots$ & $\ldots$ & $0.62 \pm 0.02$ \\
\hline 5518 [Cl III] & $0.30 \pm 0.03$ & $\ldots$ & $0.46 \pm 0.05$ & $0.28 \pm 0.02$ \\
\hline $5538[\mathrm{Cl} \mathrm{III}]$ & $0.22 \pm 0.02$ & $\ldots$ & $0.34 \pm 0.03$ & $0.40 \pm 0.03$ \\
\hline 5755 [N II] & & $\ldots$ & $0.74 \pm 0.04$ & \\
\hline $5876 \mathrm{He}_{\mathrm{I}}$ & $10.86 \pm 0.18$ & $11.01 \pm 0.23$ & $10.40 \pm 0.16$ & $8.68 \pm 0.14$ \\
\hline $6300[\mathrm{O} \mathrm{I}]$ & $1.78 \pm 0.05$ & $3.54 \pm 0.09$ & $1.66 \pm 0.04$ & $1.45 \pm 0.03$ \\
\hline 6312 [S III] & $1.60 \pm 0.05$ & $1.63 \pm 0.09$ & $0.90 \pm 0.03$ & $0.72 \pm 0.04$ \\
\hline $6363\left[\mathrm{O}_{\mathrm{I}}\right]$ & $0.59 \pm 0.03$ & $0.96 \pm 0.05$ & $0.37 \pm 0.02$ & $0.19 \pm 0.01$ \\
\hline 6548 [N II] & $0.77 \pm 0.02$ & $3.44 \pm 0.09$ & $30.84 \pm 0.49$ & $32.63 \pm 0.52$ \\
\hline $6563 \mathrm{H} \alpha$ & $280.51 \pm 4.54$ & $286.39 \pm 4.53$ & $285.57 \pm 4.50$ & $296.48 \pm 4.76$ \\
\hline 6583 [N II] & $2.40 \pm 0.06$ & $10.28 \pm 0.16$ & $94.55 \pm 1.50$ & $72.87 \pm 1.17$ \\
\hline $6678 \mathrm{He} \mathrm{I}$ & $3.23 \pm 0.06$ & $\ldots$ & $2.39 \pm 0.04$ & $2.38 \pm 0.04$ \\
\hline 6717 [S II] & $5.55 \pm 0.10$ & $\ldots$ & $14.69 \pm 0.24$ & $14.10 \pm 0.23$ \\
\hline 6731 [S II] & $4.16 \pm 0.09$ & $\ldots$ & $17.87 \pm 0.29$ & $15.94 \pm 0.26$ \\
\hline $7065 \mathrm{He}$ I & $\ldots$ & $\ldots$ & $3.06 \pm 0.05$ & $2.19 \pm 0.04$ \\
\hline 7136 [Ar III] & $\ldots$ & $\ldots$ & $8.43 \pm 0.14$ & $5.44 \pm 0.09$ \\
\hline $7281 \mathrm{He}$ I & $\cdots$ & $\cdots$ & $\ldots$ & $0.34 \pm 0.02$ \\
\hline 7320 [O II] & $\ldots$ & $\ldots$ & $3.46 \pm 0.06$ & $2.33 \pm 0.04$ \\
\hline 7330 [O II] & $\ldots$ & $\ldots$ & $2.64 \pm 0.05$ & $1.90 \pm 0.03$ \\
\hline 9069 [S III] & $\ldots$ & $\ldots$ & $34.18 \pm 0.64$ & $27.71 \pm 0.53$ \\
\hline$C(\mathrm{H} \beta)$ & 0.225 & 0.295 & 0.495 & 0.675 \\
\hline$F(\mathrm{H} \beta)^{a}$ & 113.10 & 37.45 & 2075.00 & 3627.00 \\
\hline$E W(\mathrm{H} \beta) \AA$ & 127.5 & 76.0 & 28.3 & 40.2 \\
\hline$E W($ abs $) \AA$ & 5.90 & 0.30 & 0.50 & 0.95 \\
\hline \multirow{3}{*}{ Line } & \multicolumn{4}{|c|}{$\begin{array}{c}\text { GALAXY } \\
\text { (ESO PROGRAM) }\end{array}$} \\
\hline & NGC 3125 & MRK 1259 & MRK 1271 & POX 4 \\
\hline & 31.C-0113(A)) & 073.B-0283(A)) & $(081 . \mathrm{C}-0113(\mathrm{~A}))$ & $(081 . \mathrm{C}-0113(\mathrm{~A}))$ \\
\hline $3727[\mathrm{O}$ II] & $93.12 \pm 1.44$ & $170.87 \pm 2.75$ & $104.71 \pm 1.63$ & $81.26 \pm 1.27$ \\
\hline $3750 \mathrm{H} 12$ & $3.65 \pm 0.10$ & $\ldots$ & $3.84 \pm 0.08$ & $5.50 \pm 0.10$ \\
\hline $3771 \mathrm{H} 11$ & $4.35 \pm 0.11$ & $\ldots$ & $4.43 \pm 0.09$ & $6.14 \pm 0.11$ \\
\hline $3798 \mathrm{H} 10$ & $5.55 \pm 0.14$ & $7.61 \pm 0.64$ & $5.50 \pm 0.11$ & $7.31 \pm 0.12$ \\
\hline $3820 \mathrm{He}_{\mathrm{I}}$ & $1.14 \pm 0.04$ & $\ldots$ & $0.87 \pm 0.03$ & $0.76 \pm 0.02$ \\
\hline 3835 H9 & $7.72 \pm 0.14$ & $9.29 \pm 0.58$ & $7.37 \pm 0.12$ & $9.32 \pm 0.15$ \\
\hline 3868 [Ne III] & $55.82 \pm 0.85$ & $6.66 \pm 0.22$ & $52.59 \pm 0.80$ & $44.61 \pm 0.68$ \\
\hline $3889 \mathrm{He}_{\mathrm{I}}+\mathrm{H} 8$ & $22.86 \pm 0.36$ & $17.30 \pm 0.42$ & $18.36 \pm 0.28$ & $17.36 \pm 0.27$ \\
\hline 3968 [Ne III] + H7 & $35.10 \pm 0.53$ & $14.21 \pm 0.41$ & $36.82 \pm 0.56$ & $29.19 \pm 0.44$ \\
\hline $4026 \mathrm{He}_{\mathrm{I}}$ & $2.17 \pm 0.07$ & $\ldots$ & $1.46 \pm 0.03$ & $1.59 \pm 0.03$ \\
\hline 4068 [S II] & $0.80 \pm 0.02$ & $2.07 \pm 0.14$ & $0.75 \pm 0.02$ & $0.73 \pm 0.02$ \\
\hline 4076 [S II] & $0.27 \pm 0.01$ & $\ldots$ & $0.19 \pm 0.01$ & $0.26 \pm 0.01$ \\
\hline $4101 \mathrm{H} \delta$ & $29.87 \pm 0.45$ & $24.37 \pm 0.45$ & $25.98 \pm 0.39$ & $27.12 \pm 0.41$ \\
\hline $4227[\mathrm{Fe} \mathrm{v}]$ & & & 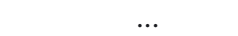 & $2.09 \pm 0.57$ \\
\hline $4340 \mathrm{H} \gamma$ & $59.38 \pm 0.86$ & $46.45 \pm 0.74$ & $47.02 \pm 0.69$ & $46.28 \pm 0.68$ \\
\hline 4363 [O III] & $5.13 \pm 0.08$ & $1.08 \pm 0.12$ & $8.31 \pm 0.12$ & $9.12 \pm 0.13$ \\
\hline $4387 \mathrm{He}_{\mathrm{I}}$ & $0.64 \pm 0.02$ & $\ldots$ & $0.39 \pm 0.01$ & $0.37 \pm 0.01$ \\
\hline $4471 \mathrm{He}_{\mathrm{I}}$ & $5.03 \pm 0.07$ & $2.92 \pm 0.13$ & $3.22 \pm 0.05$ & $3.34 \pm 0.05$ \\
\hline 4658 [Fe III] & $0.56 \pm 0.02$ & $\ldots$ & $0.45 \pm 0.01$ & $0.47 \pm 0.01$ \\
\hline
\end{tabular}


Table 3. continued.

\begin{tabular}{|c|c|c|c|c|}
\hline \multirow{3}{*}{ Line } & \multicolumn{4}{|c|}{$\begin{array}{c}\text { GALAXY } \\
\text { (ESO PROGRAM) }\end{array}$} \\
\hline & NGC 3125 & MRK 1259 & MRK 1271 & POX 4 \\
\hline & $(081 . \mathrm{C}-0113(\mathrm{~A}))$ & $(073 . \mathrm{B}-0283(\mathrm{~A}))$ & $(081 . \mathrm{C}-0113(\mathrm{~A}))$ & $(081 . \mathrm{C}-0113(\mathrm{~A}))$ \\
\hline $4686 \mathrm{He}_{\mathrm{II}}$ & $4.47 \pm 0.15$ & $\ldots$ & $0.16 \pm 0.01$ & $0.71 \pm 0.02$ \\
\hline $4711[\mathrm{Ar}$ IV $]+\mathrm{He}_{\mathrm{I}}$ & $1.14 \pm 0.03$ & $\ldots$ & $1.30 \pm 0.02$ & $1.54 \pm 0.02$ \\
\hline 4740 [Ar IV] & $0.44 \pm 0.02$ & $\ldots$ & $0.70 \pm 0.01$ & $0.93 \pm 0.02$ \\
\hline $4861 \mathrm{H} \beta$ & $100.00 \pm 1.43$ & $100.00 \pm 1.49$ & $100.00 \pm 1.44$ & $100.00 \pm 1.44$ \\
\hline $4921 \mathrm{He}_{\mathrm{I}}$ & $0.99 \pm 0.03$ & $\ldots$ & $0.91 \pm 0.03$ & $0.90 \pm 0.03$ \\
\hline 4959 [O III] & $197.05 \pm 2.81$ & $55.21 \pm 0.83$ & $187.87 \pm 2.70$ & $218.42 \pm 3.15$ \\
\hline 5007 [O III] & $578.95 \pm 8.27$ & $161.78 \pm 2.41$ & $555.77 \pm 7.98$ & $652.36 \pm 9.40$ \\
\hline $5015 \mathrm{He}_{\mathrm{I}}$ & $2.01 \pm 0.04$ & $\ldots$ & $1.57 \pm 0.03$ & $1.77 \pm 0.03$ \\
\hline $5199\left[\mathrm{~N}_{\mathrm{I}}\right]$ & $0.36 \pm 0.01$ & $\ldots$ & $0.27 \pm 0.01$ & $0.24 \pm 0.01$ \\
\hline $5518[\mathrm{Cl} \mathrm{III}]$ & $0.37 \pm 0.02$ & $\ldots$ & $0.24 \pm 0.01$ & $0.32 \pm 0.01$ \\
\hline $5538[\mathrm{Cl} \mathrm{III}]$ & $0.27 \pm 0.01$ & $\ldots$ & $0.16 \pm 0.01$ & $0.22 \pm 0.01$ \\
\hline $5755\left[\mathrm{~N}_{\text {II }}\right]$ & & $0.65 \pm 0.08$ & & \\
\hline $5876 \mathrm{He}_{\mathrm{I}}$ & $15.57 \pm 0.23$ & $13.66 \pm 0.22$ & $7.62 \pm 0.12$ & $10.10 \pm 0.15$ \\
\hline $6300[\mathrm{O} \mathrm{I}]$ & $2.18 \pm 0.04$ & $2.70 \pm 0.06$ & $2.39 \pm 0.04$ & $1.79 \pm 0.03$ \\
\hline $6312\left[\mathrm{~S}_{\mathrm{III}}\right]$ & $2.06 \pm 0.04$ & $0.77 \pm 0.03$ & $1.27 \pm 0.02$ & $1.64 \pm 0.03$ \\
\hline $6363\left[\mathrm{O}_{\mathrm{I}}\right]$ & $0.78 \pm 0.02$ & $0.83 \pm 0.04$ & $0.69 \pm 0.02$ & $0.60 \pm 0.01$ \\
\hline $6548[\mathrm{~N}$ II $]$ & $3.41 \pm 0.05$ & $23.82 \pm 0.39$ & $1.48 \pm 0.03$ & $1.32 \pm 0.02$ \\
\hline $6563 \mathrm{H} \alpha$ & $287.50 \pm 4.45$ & $290.54 \pm 4.69$ & $180.14 \pm 2.81$ & $283.03 \pm 4.42$ \\
\hline $6583[\mathrm{~N}$ II] & $10.64 \pm 0.17$ & $84.92 \pm 1.38$ & $4.67 \pm 0.08$ & $3.49 \pm 0.06$ \\
\hline $6678 \mathrm{He}_{\mathrm{I}}$ & $4.91 \pm 0.08$ & $2.78 \pm 0.05$ & $2.47 \pm 0.04$ & $2.89 \pm 0.05$ \\
\hline $6717[\mathrm{~S} \mathrm{II}]$ & $11.97 \pm 0.19$ & $15.75 \pm 0.26$ & $8.94 \pm 0.14$ & $7.63 \pm 0.12$ \\
\hline $6731[\mathrm{~S}$ II] & $9.92 \pm 0.16$ & $18.13 \pm 0.30$ & $6.69 \pm 0.11$ & $5.94 \pm 0.10$ \\
\hline $7065 \mathrm{He} \mathrm{I}$ & $4.28 \pm 0.07$ & $3.37 \pm 0.06$ & $2.39 \pm 0.04$ & $2.33 \pm 0.04$ \\
\hline 7136 [Ar III] & $13.00 \pm 0.21$ & $5.88 \pm 0.10$ & $6.10 \pm 0.10$ & $5.35 \pm 0.09$ \\
\hline $7281 \mathrm{He}_{\mathrm{I}}$ & $0.74 \pm 0.02$ & & $0.46 \pm 0.01$ & $0.48 \pm 0.01$ \\
\hline $7320[\mathrm{O}$ II] & $2.42 \pm 0.04$ & $3.76 \pm 0.07$ & $1.49 \pm 0.03$ & $1.32 \pm 0.02$ \\
\hline $7330[\mathrm{O}$ II] & $1.75 \pm 0.03$ & $2.97 \pm 0.06$ & $1.25 \pm 0.02$ & $0.96 \pm 0.02$ \\
\hline 9069 [S III] & $31.97 \pm 0.59$ & $23.74 \pm 0.46$ & $2.23 \pm 0.04$ & $12.31 \pm 0.23$ \\
\hline$C(\mathrm{H} \beta)$ & 0.390 & 0.660 & 0.190 & 0.170 \\
\hline$F(\mathrm{H} \beta)^{a}$ & 863.30 & 1025.00 & 1368.00 & 1457.00 \\
\hline$E W(\mathrm{H} \beta) \AA$ & 146.2 & 41.5 & 167.6 & 228.4 \\
\hline$E W(\mathrm{abs}) \AA$ & 0.30 & 1.55 & 1.75 & 3.35 \\
\hline \multirow{3}{*}{ Line } & \multicolumn{4}{|c|}{$\begin{array}{c}\text { GALAXY } \\
\text { (ESO PROGRAM) }\end{array}$} \\
\hline & TOL 1214-277 & J1253-0312 & NGC 5253 No.C1 & NGC 5253 No.C2 \\
\hline & (69.D-0174(A)) & (081.C-0113(A)) & (073.B-0283(A)) & (073.B-0283(A)) \\
\hline $3346[\mathrm{Ne} \mathrm{v}]$ & $1.15 \pm 0.02$ & $\ldots$ & $\ldots$ & $\ldots$ \\
\hline $3425[\mathrm{Ne} \mathrm{v}]$ & $3.76 \pm 0.13$ & $\ldots$ & $\ldots$ & $\ldots$ \\
\hline $3727[\mathrm{O}$ II] & $30.82 \pm 0.48$ & $84.22 \pm 1.30$ & $96.34 \pm 1.50$ & $148.48 \pm 2.32$ \\
\hline $3750 \mathrm{H} 12$ & $3.98 \pm 0.10$ & $3.98 \pm 0.07$ & $3.77 \pm 0.07$ & $4.16 \pm 0.10$ \\
\hline $3771 \mathrm{H} 11$ & $4.25 \pm 0.11$ & $4.39 \pm 0.07$ & $4.38 \pm 0.08$ & $4.61 \pm 0.10$ \\
\hline 3798 H10 & $7.29 \pm 0.14$ & $6.57 \pm 0.10$ & $6.18 \pm 0.10$ & $5.82 \pm 0.11$ \\
\hline $3820 \mathrm{He}_{\mathrm{I}}$ & $1.00 \pm 0.05$ & $0.94 \pm 0.03$ & $0.97 \pm 0.02$ & $0.94 \pm 0.04$ \\
\hline 3835 H9 & $7.29 \pm 0.15$ & $7.19 \pm 0.11$ & $7.97 \pm 0.13$ & $7.59 \pm 0.14$ \\
\hline 3868 [Ne III] & $41.33 \pm 0.64$ & $47.19 \pm 0.71$ & $55.40 \pm 0.84$ & $26.29 \pm 0.40$ \\
\hline $3889 \mathrm{He}_{\mathrm{I}}+\mathrm{H} 8$ & $21.28 \pm 0.33$ & $18.76 \pm 0.28$ & $17.40 \pm 0.27$ & $17.84 \pm 0.28$ \\
\hline $3968[\mathrm{Ne}$ III $]+\mathrm{H} 7$ & $\ldots$ & $33.24 \pm 0.50$ & $35.35 \pm 0.53$ & $\ldots$ \\
\hline $4026 \mathrm{He}_{\mathrm{I}}$ & $1.88 \pm 0.05$ & $1.62 \pm 0.03$ & $1.93 \pm 0.03$ & $1.67 \pm 0.04$ \\
\hline $4068[\mathrm{~S}$ II $]$ & $\ldots$ & $1.08 \pm 0.02$ & $1.39 \pm 0.03$ & $1.16 \pm 0.04$ \\
\hline $4076\left[\mathrm{~S}_{\mathrm{II}}\right]$ & $\ldots$ & $0.34 \pm 0.01$ & $0.49 \pm 0.01$ & $0.46 \pm 0.03$ \\
\hline $4101 \mathrm{H} \delta$ & $29.39 \pm 0.44$ & $27.32 \pm 0.40$ & $25.49 \pm 0.38$ & $25.71 \pm 0.39$ \\
\hline $4227[\mathrm{Fe} \mathrm{v}]$ & $0.80 \pm 0.07$ & $0.63 \pm 0.05$ & $\ldots$ & $\ldots$ \\
\hline $4340 \mathrm{H} \gamma$ & $50.93 \pm 0.74$ & $48.28 \pm 0.70$ & $48.56 \pm 0.71$ & $46.62 \pm 0.68$ \\
\hline $4363[\mathrm{O} \mathrm{III}]$ & $17.29 \pm 0.26$ & $10.37 \pm 0.15$ & $7.45 \pm 0.11$ & $3.09 \pm 0.05$ \\
\hline $4387 \mathrm{He}_{\mathrm{I}}$ & $0.46 \pm 0.03$ & $0.40 \pm 0.01$ & $0.44 \pm 0.01$ & $0.42 \pm 0.02$ \\
\hline $4471 \mathrm{He}_{\mathrm{I}}$ & $3.63 \pm 0.07$ & $4.15 \pm 0.06$ & $4.53 \pm 0.07$ & $3.95 \pm 0.06$ \\
\hline $4658[\mathrm{Fe}$ III] & $0.26 \pm 0.05$ & $0.91 \pm 0.01$ & $0.83 \pm 0.01$ & $0.79 \pm 0.02$ \\
\hline 4686 He II & $4.93 \pm 0.09$ & $0.65 \pm 0.01$ & $\ldots$ & \\
\hline $4711[\mathrm{Ar}$ Iv $]+\mathrm{He}_{\mathrm{I}}$ & $\ldots$ & $1.75 \pm 0.03$ & $\ldots$ & $0.77 \pm 0.03$ \\
\hline $4740[\mathrm{Ar} \mathrm{IV}]$ & $2.07 \pm 0.05$ & $0.99 \pm 0.02$ & $1.27 \pm 0.02$ & $0.18 \pm 0.01$ \\
\hline $4861 \mathrm{H} \beta$ & $100.00 \pm 1.43$ & $100.00 \pm 1.43$ & $100.00 \pm 1.43$ & $100.00 \pm 1.43$ \\
\hline $4921 \mathrm{He}_{\mathrm{I}}$ & $0.85 \pm 0.02$ & $0.80 \pm 0.02$ & $0.98 \pm 0.02$ & $0.91 \pm 0.02$ \\
\hline
\end{tabular}


Table 3. continued.

\begin{tabular}{|c|c|c|c|c|}
\hline \multirow{3}{*}{ Line } & \multicolumn{4}{|c|}{$\begin{array}{c}\text { GALAXY } \\
\text { (ESO PROGRAM) }\end{array}$} \\
\hline & TOL 1214-277 & $\mathrm{J} 1253-0312$ & NGC 5253 No.C1 & NGC 5253 No.C2 \\
\hline & $(69 . \mathrm{D}-0174(\mathrm{~A}))$ & $(081 . \mathrm{C}-0113(\mathrm{~A}))$ & $(073 . \mathrm{B}-0283(\mathrm{~A}))$ & $(073 . \mathrm{B}-0283(\mathrm{~A}))$ \\
\hline 4959 [O III] & $171.89 \pm 2.45$ & $192.25 \pm 2.74$ & $206.60 \pm 2.95$ & $149.13 \pm 2.13$ \\
\hline $5007[\mathrm{O} \mathrm{III}]$ & $511.82 \pm 7.29$ & $665.63 \pm 9.50$ & $634.27 \pm 9.07$ & $445.98 \pm 6.39$ \\
\hline $5015 \mathrm{He}_{\mathrm{I}}$ & $1.54 \pm 0.03$ & $3.15 \pm 0.05$ & $1.97 \pm 0.03$ & $2.05 \pm 0.04$ \\
\hline $5199\left[\mathrm{~N} \mathrm{I}_{\mathrm{I}}\right]$ & $\ldots$ & $0.37 \pm 0.01$ & $\ldots$ & $\ldots$ \\
\hline $5518[\mathrm{Cl} \mathrm{III}]$ & $\ldots$ & $0.30 \pm 0.01$ & $0.34 \pm 0.01$ & $0.34 \pm 0.02$ \\
\hline $5538[\mathrm{Cl} \mathrm{III}]$ & $\ldots$ & $0.17 \pm 0.01$ & $0.29 \pm 0.01$ & $0.28 \pm 0.01$ \\
\hline $5755\left[\mathrm{~N}_{\text {II }}\right]$ & $\ldots$ & $0.32 \pm 0.01$ & $0.44 \pm 0.01$ & $0.24 \pm 0.02$ \\
\hline $5876 \mathrm{He}_{\mathrm{I}}$ & $9.02 \pm 0.14$ & $5.52 \pm 0.08$ & $12.33 \pm 0.18$ & $11.15 \pm 0.17$ \\
\hline $6300[\mathrm{O} \mathrm{I}]$ & $0.86 \pm 0.03$ & $2.26 \pm 0.04$ & $1.83 \pm 0.03$ & $2.25 \pm 0.04$ \\
\hline $6312[\mathrm{~S}$ III] & $0.83 \pm 0.03$ & $1.49 \pm 0.03$ & $2.32 \pm 0.04$ & $1.98 \pm 0.04$ \\
\hline $6363[\mathrm{O} \mathrm{I}]$ & $0.29 \pm 0.03$ & $0.64 \pm 0.01$ & $0.58 \pm 0.01$ & $0.70 \pm 0.02$ \\
\hline 6548 [N II] & $0.29 \pm 0.02$ & $5.65 \pm 0.09$ & $7.52 \pm 0.12$ & $4.48 \pm 0.07$ \\
\hline $6563 \mathrm{H} \alpha$ & $275.03 \pm 4.25$ & $282.29 \pm 4.37$ & $285.29 \pm 4.42$ & $287.86 \pm 4.47$ \\
\hline 6583 [N II] & $0.84 \pm 0.03$ & $13.15 \pm 0.20$ & $21.43 \pm 0.33$ & $12.55 \pm 0.20$ \\
\hline $6678 \mathrm{He}_{\mathrm{I}}$ & $2.40 \pm 0.04$ & $2.40 \pm 0.04$ & $3.57 \pm 0.06$ & $3.78 \pm 0.06$ \\
\hline $6717\left[\mathrm{~S}_{\mathrm{II}}\right]$ & $2.24 \pm 0.05$ & $4.94 \pm 0.08$ & $7.79 \pm 0.12$ & $14.80 \pm 0.23$ \\
\hline $6731[\mathrm{~S}$ II] & $1.39 \pm 0.04$ & $6.25 \pm 0.10$ & $7.79 \pm 0.12$ & $12.20 \pm 0.19$ \\
\hline $7065 \mathrm{He}_{\mathrm{I}}$ & $2.56 \pm 0.04$ & $4.31 \pm 0.07$ & $6.04 \pm 0.10$ & $3.42 \pm 0.06$ \\
\hline 7136 [Ar III] & $2.08 \pm 0.04$ & $6.65 \pm 0.11$ & $12.68 \pm 0.21$ & $11.31 \pm 0.18$ \\
\hline $7281 \mathrm{He}_{\mathrm{I}}$ & $0.59 \pm 0.02$ & $0.48 \pm 0.01$ & $0.58 \pm 0.01$ & $0.58 \pm 0.01$ \\
\hline 7320 [O II] & $0.50 \pm 0.02$ & $1.85 \pm 0.03$ & $2.33 \pm 0.04$ & $2.57 \pm 0.04$ \\
\hline $7330[\mathrm{O}$ II $]$ & $0.48 \pm 0.02$ & $1.49 \pm 0.03$ & $1.86 \pm 0.03$ & $2.10 \pm 0.04$ \\
\hline 9069 [S III] & $2.85 \pm 0.12$ & $13.74 \pm 0.25$ & $22.44 \pm 0.41$ & $21.57 \pm 0.40$ \\
\hline$C(\mathrm{H} \beta)$ & 0.285 & 0.265 & 0.380 & 0.125 \\
\hline$F(\mathrm{H} \beta)^{a}$ & 700.80 & 1834.00 & 2559.00 & 1019.00 \\
\hline$E W(\mathrm{H} \beta) \AA$ & 335.8 & 236.7 & 526.8 & 118.8 \\
\hline$E W($ abs $) \AA$ & 0.05 & 0.90 & 1.60 & 0.80 \\
\hline \multirow{3}{*}{ Line } & \multicolumn{4}{|c|}{$\begin{array}{c}\text { GALAXY } \\
\text { (ESO PROGRAM) }\end{array}$} \\
\hline & VGC 5253 No.P1 & NGC 5253 No.P2 & TOL 89 No.1 & TOL 89 No.2 \\
\hline & $(70 . \mathrm{C}-0008(\mathrm{~A}))$ & (70.C-0008(A)) & $(073 . \mathrm{B}-0283(\mathrm{~A}))$ & $(073 . \mathrm{B}-0283(\mathrm{~A}))$ \\
\hline 3727 [O II] & $175.56 \pm 2.77$ & $106.83 \pm 1.69$ & $151.60 \pm 2.36$ & $157.62 \pm 2.60$ \\
\hline $3750 \mathrm{H} 12$ & $3.62 \pm 0.08$ & $3.34 \pm 0.07$ & $3.39 \pm 0.08$ & $10.82 \pm 0.51$ \\
\hline 3771 H11 & $4.65 \pm 0.10$ & $4.18 \pm 0.09$ & $4.47 \pm 0.09$ & $11.41 \pm 0.44$ \\
\hline 3798 H10 & $6.61 \pm 0.12$ & $6.41 \pm 0.11$ & $5.93 \pm 0.11$ & $12.30 \pm 0.44$ \\
\hline $3820 \mathrm{He}_{\mathrm{I}}$ & $1.27 \pm 0.04$ & $1.07 \pm 0.03$ & $1.19 \pm 0.05$ & $\cdots$ \\
\hline $3835 \mathrm{H} 9$ & $9.13 \pm 0.16$ & $9.01 \pm 0.15$ & $8.04 \pm 0.14$ & $15.45 \pm 0.42$ \\
\hline 3868 [Ne III] & $40.59 \pm 0.63$ & $61.05 \pm 0.93$ & $33.83 \pm 0.52$ & $17.85 \pm 0.31$ \\
\hline $3889 \mathrm{He}_{\mathrm{I}}+\mathrm{H} 8$ & $22.36 \pm 0.35$ & $21.28 \pm 0.33$ & $19.96 \pm 0.32$ & $23.36 \pm 0.46$ \\
\hline $3968[\mathrm{Ne}$ III] + H7 & $\ldots$ & $34.85 \pm 0.53$ & $\ldots$ & $\ldots$ \\
\hline $4026 \mathrm{He}_{\mathrm{I}}$ & $2.05 \pm 0.05$ & $2.46 \pm 0.05$ & $1.89 \pm 0.05$ & $1.24 \pm 0.10$ \\
\hline $4068[\mathrm{~S}$ II $]$ & $1.32 \pm 0.05$ & $1.55 \pm 0.04$ & $0.98 \pm 0.03$ & $\ldots$ \\
\hline $4076[\mathrm{~S}$ II $]$ & $0.61 \pm 0.05$ & $0.57 \pm 0.03$ & $0.31 \pm 0.02$ & $\ldots$ \\
\hline $4101 \mathrm{H} \delta$ & $29.86 \pm 0.45$ & $28.71 \pm 0.43$ & $25.47 \pm 0.39$ & $26.04 \pm 0.44$ \\
\hline $4340 \mathrm{H} \gamma$ & $52.64 \pm 0.77$ & $47.32 \pm 0.69$ & $50.24 \pm 0.73$ & $45.75 \pm 0.71$ \\
\hline 4363 [O III] & $\ldots$ & $7.92 \pm 0.12$ & $3.38 \pm 0.07$ & $1.94 \pm 0.11$ \\
\hline $4387 \mathrm{He}_{\mathrm{I}}$ & $0.49 \pm 0.02$ & $0.62 \pm 0.02$ & $0.45 \pm 0.02$ & 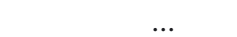 \\
\hline $4471 \mathrm{He}_{\mathrm{I}}$ & $4.70 \pm 0.07$ & $4.71 \pm 0.07$ & $4.62 \pm 0.07$ & $3.42 \pm 0.08$ \\
\hline $4658[\mathrm{Fe}$ III] & $0.68 \pm 0.02$ & $0.88 \pm 0.02$ & $0.51 \pm 0.02$ & $0.77 \pm 0.07$ \\
\hline $4711[$ Ar Iv $]+\mathrm{He}_{\mathrm{I}}$ & $0.76 \pm 0.04$ & $\ldots$ & $0.70 \pm 0.02$ & $\ldots$ \\
\hline 4740 [Ar Iv] & $0.14 \pm 0.02$ & $0.97 \pm 0.03$ & $0.20 \pm 0.01$ & $\ldots$ \\
\hline $4861 \mathrm{H} \beta$ & $100.00 \pm 1.43$ & $100.00 \pm 1.43$ & $100.00 \pm 1.43$ & $100.00 \pm 1.50$ \\
\hline $4921 \mathrm{He} \mathrm{I}$ & $1.05 \pm 0.03$ & $1.15 \pm 0.03$ & $1.13 \pm 0.02$ & $1.08 \pm 0.07$ \\
\hline 4959 [O III] & $170.25 \pm 2.43$ & $217.38 \pm 3.10$ & $161.11 \pm 2.30$ & $122.61 \pm 1.84$ \\
\hline $5007[\mathrm{O} \mathrm{III}]$ & $505.23 \pm 7.20$ & $649.56 \pm 9.26$ & $489.58 \pm 6.99$ & $385.19 \pm 5.76$ \\
\hline $5015 \mathrm{He}_{\mathrm{I}}$ & $2.31 \pm 0.05$ & $2.09 \pm 0.04$ & $2.19 \pm 0.04$ & $1.97 \pm 0.07$ \\
\hline 5518 [Cl III] & $0.34 \pm 0.04$ & $0.31 \pm 0.02$ & $0.41 \pm 0.01$ & $0.40 \pm 0.05$ \\
\hline $5538[\mathrm{Cl} \mathrm{III}]$ & $0.37 \pm 0.03$ & $0.31 \pm 0.02$ & $0.30 \pm 0.01$ & $\ldots$ \\
\hline $5755[\mathrm{~N}$ II $]$ & $\ldots$ & $0.56 \pm 0.02$ & $0.23 \pm 0.02$ & $\ldots$ \\
\hline $5876 \mathrm{He}_{\mathrm{I}}$ & $11.24 \pm 0.17$ & $12.56 \pm 0.19$ & $12.45 \pm 0.19$ & $11.59 \pm 0.22$ \\
\hline 6300 [O I $]$ & $1.62 \pm 0.04$ & $1.64 \pm 0.03$ & $1.62 \pm 0.03$ & $1.01 \pm 0.08$ \\
\hline 6312 [S III] & $1.85 \pm 0.05$ & $2.53 \pm 0.05$ & $1.69 \pm 0.03$ & $1.38 \pm 0.08$ \\
\hline
\end{tabular}


Table 3. continued.

\begin{tabular}{|c|c|c|c|c|}
\hline \multirow[b]{2}{*}{ Line } & \multicolumn{4}{|c|}{$\begin{array}{c}\text { GALAXY } \\
\text { (ESO PROGRAM) }\end{array}$} \\
\hline & $\begin{array}{l}\text { NGC } 5253 \text { No.P1 } \\
(70 . C-0008(A))\end{array}$ & $\begin{array}{r}\text { NGC } 5253 \text { No.P2 } \\
(70 . C-0008(A))\end{array}$ & $\begin{array}{r}\text { TOL 89 No.1 } \\
(073 . \mathrm{B}-0283(\mathrm{~A}))\end{array}$ & $\begin{array}{r}\text { TOL 89 No.2 } \\
(073 . \mathrm{B}-0283(\mathrm{~A}))\end{array}$ \\
\hline $6363[\mathrm{O} \mathrm{I}]$ & $0.57 \pm 0.02$ & $0.42 \pm 0.01$ & $0.53 \pm 0.02$ & \\
\hline $6548[\mathrm{~N}$ II $]$ & $3.47 \pm 0.06$ & $10.35 \pm 0.16$ & $4.42 \pm 0.07$ & $4.67 \pm 0.09$ \\
\hline $6563 \mathrm{H} \alpha$ & $288.25 \pm 4.46$ & $284.86 \pm 4.41$ & $288.74 \pm 4.47$ & $289.64 \pm 4.70$ \\
\hline $6583[\mathrm{~N}$ II] & $9.54 \pm 0.15$ & $27.65 \pm 0.43$ & $13.32 \pm 0.21$ & $13.81 \pm 0.25$ \\
\hline $6678 \mathrm{He}_{\mathrm{I}}$ & $3.30 \pm 0.05$ & $3.34 \pm 0.05$ & $3.54 \pm 0.06$ & $6.97 \pm 0.12$ \\
\hline 6717 [S II] & $10.10 \pm 0.16$ & $7.19 \pm 0.11$ & $11.67 \pm 0.18$ & $24.69 \pm 0.41$ \\
\hline $6731[\mathrm{~S}$ II] & $8.83 \pm 0.14$ & $8.00 \pm 0.13$ & $8.99 \pm 0.14$ & $18.48 \pm 0.31$ \\
\hline $7065 \mathrm{He}_{\mathrm{I}}$ & $3.31 \pm 0.05$ & $5.22 \pm 0.08$ & $3.01 \pm 0.05$ & $5.68 \pm 0.10$ \\
\hline 7136 [Ar III] & $10.85 \pm 0.18$ & $12.88 \pm 0.21$ & $10.65 \pm 0.17$ & $21.88 \pm 0.38$ \\
\hline $7281 \mathrm{He}_{\mathrm{I}}$ & $0.54 \pm 0.01$ & $0.63 \pm 0.01$ & $0.61 \pm 0.02$ & $1.42 \pm 0.06$ \\
\hline 7320 [O II] & $2.41 \pm 0.04$ & $2.48 \pm 0.04$ & $1.93 \pm 0.03$ & $4.34 \pm 0.09$ \\
\hline $7330[\mathrm{O}$ II] & $1.93 \pm 0.03$ & $1.96 \pm 0.03$ & $1.58 \pm 0.03$ & $3.29 \pm 0.07$ \\
\hline 9069 [S III] & $21.11 \pm 0.39$ & $22.82 \pm 0.42$ & $24.83 \pm 0.46$ & $50.12 \pm 1.00$ \\
\hline$C(\mathrm{H} \beta)$ & 0.180 & 0.335 & 0.330 & 0.105 \\
\hline$F(\mathrm{H} \beta)^{a}$ & 1954.00 & 3265.00 & 341.90 & 174.80 \\
\hline$E W(\mathrm{H} \beta) \AA$ & 122.3 & 900.0 & 313.8 & 70.2 \\
\hline$E W(\mathrm{abs}) \AA$ & 0.10 & 0.50 & 0.45 & 3.25 \\
\hline \multirow{3}{*}{ Line } & \multicolumn{4}{|c|}{$\begin{array}{c}\text { GALAXY } \\
\text { ESO PROGRAM) }\end{array}$} \\
\hline & NGC 5408 No.1 & NGC 5408 No.2 & TOL 1457-262 & TOL 1924-416 No.1 \\
\hline & (081.C-0113(A)) & $(081 . \mathrm{C}-0113(\mathrm{~A}))$ & (081.C-0113(A)) & (081.C-0113(A)) \\
\hline 3727 [O II] & $116.51 \pm 1.80$ & $196.84 \pm 3.04$ & $258.97 \pm 4.03$ & $94.48 \pm 1.55$ \\
\hline $3750 \mathrm{H} 12$ & $5.09 \pm 0.10$ & $5.08 \pm 0.13$ & $4.30 \pm 0.09$ & $12.55 \pm 0.39$ \\
\hline 3771 H11 & $6.65 \pm 0.13$ & $6.42 \pm 0.16$ & $6.09 \pm 0.12$ & $12.98 \pm 0.35$ \\
\hline 3798 H10 & $8.67 \pm 0.15$ & $7.93 \pm 0.16$ & $8.05 \pm 0.14$ & $14.27 \pm 0.37$ \\
\hline $3820 \mathrm{He}_{\mathrm{I}}$ & $1.74 \pm 0.03$ & $1.45 \pm 0.06$ & $1.49 \pm 0.05$ & $0.44 \pm 0.03$ \\
\hline 3835 H9 & $11.95 \pm 0.19$ & $11.15 \pm 0.23$ & $11.34 \pm 0.19$ & $14.56 \pm 0.36$ \\
\hline 3868 [Ne III] & $85.90 \pm 1.30$ & $60.79 \pm 0.92$ & $50.36 \pm 0.77$ & $30.70 \pm 0.50$ \\
\hline 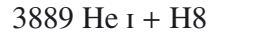 & $33.23 \pm 0.50$ & $30.67 \pm 0.47$ & $34.24 \pm 0.53$ & $21.82 \pm 0.36$ \\
\hline $3968[\mathrm{Ne}$ III] + H7 & $53.05 \pm 0.80$ & $47.46 \pm 0.72$ & $38.78 \pm 0.59$ & $26.38 \pm 0.48$ \\
\hline $4026 \mathrm{He}_{\mathrm{I}}$ & $2.68 \pm 0.04$ & $2.22 \pm 0.04$ & $2.17 \pm 0.04$ & $0.93 \pm 0.02$ \\
\hline 4068 [S II] & $0.95 \pm 0.03$ & $1.19 \pm 0.03$ & $1.92 \pm 0.05$ & $0.59 \pm 0.03$ \\
\hline 4076 [S II] & $0.25 \pm 0.01$ & $0.49 \pm 0.03$ & $0.68 \pm 0.02$ & $0.15 \pm 0.01$ \\
\hline $4101 \mathrm{H} \delta$ & $37.75 \pm 0.56$ & $32.26 \pm 0.48$ & $38.31 \pm 0.57$ & $30.96 \pm 0.49$ \\
\hline $4227[\mathrm{Fe} \mathrm{v}]$ & $0.15 \pm 0.03$ & $\ldots$ & $\ldots$ & $9.24 \pm 4.35$ \\
\hline $4340 \mathrm{H} \gamma$ & $76.17 \pm 1.11$ & $71.39 \pm 1.04$ & $64.31 \pm 0.94$ & $44.03 \pm 0.68$ \\
\hline $4363[\mathrm{O} \mathrm{III}]$ & $19.77 \pm 0.29$ & $11.91 \pm 0.18$ & $6.80 \pm 0.10$ & $5.36 \pm 0.09$ \\
\hline $4387 \mathrm{He}_{\mathrm{I}}$ & $0.72 \pm 0.01$ & $0.61 \pm 0.02$ & $0.51 \pm 0.03$ & $0.18 \pm 0.02$ \\
\hline $4471 \mathrm{He}_{\mathrm{I}}$ & $5.83 \pm 0.08$ & $5.50 \pm 0.08$ & $4.78 \pm 0.08$ & $2.40 \pm 0.05$ \\
\hline $4658[\mathrm{Fe} \mathrm{III}]$ & $0.27 \pm 0.01$ & $0.24 \pm 0.01$ & $1.68 \pm 0.07$ & $0.78 \pm 0.02$ \\
\hline $4686 \mathrm{He}$ II & $1.23 \pm 0.02$ & $0.60 \pm 0.02$ & $0.66 \pm 0.02$ & $1.57 \pm 0.04$ \\
\hline $4711[\mathrm{Ar}$ IV $]+\mathrm{He}_{\mathrm{I}}$ & $3.26 \pm 0.05$ & $1.21 \pm 0.03$ & $1.23 \pm 0.04$ & $0.64 \pm 0.02$ \\
\hline $4740[\mathrm{Ar} \mathrm{IV}]$ & $\ldots$ & ... & $0.42 \pm 0.02$ & $0.28 \pm 0.01$ \\
\hline $4861 \mathrm{H} \beta$ & $100.00 \pm 1.43$ & $100.00 \pm 1.43$ & $100.00 \pm 1.44$ & $100.00 \pm 1.52$ \\
\hline $4921 \mathrm{He}_{\mathrm{I}}$ & $1.64 \pm 0.04$ & $1.76 \pm 0.04$ & $0.90 \pm 0.03$ & $0.57 \pm 0.02$ \\
\hline 4959 [O III] & $326.29 \pm 4.66$ & $197.64 \pm 2.82$ & $211.45 \pm 3.05$ & $143.04 \pm 2.17$ \\
\hline 5007 [O III] & $927.40 \pm 12.14$ & $822.91 \pm 11.75$ & $627.73 \pm 9.04$ & $436.15 \pm 6.63$ \\
\hline $5015 \mathrm{He}_{\mathrm{I}}$ & $2.82 \pm 0.05$ & $4.31 \pm 0.08$ & $1.94 \pm 0.04$ & $1.34 \pm 0.03$ \\
\hline $5199[\mathrm{~N} \mathrm{I}]$ & $0.39 \pm 0.01$ & $0.49 \pm 0.02$ & $0.70 \pm 0.02$ & $0.48 \pm 0.01$ \\
\hline 5518 [Cl III] & $0.36 \pm 0.03$ & $0.60 \pm 0.04$ & $0.39 \pm 0.02$ & $0.28 \pm 0.02$ \\
\hline $5538[\mathrm{Cl}$ III] & $0.30 \pm 0.03$ & $0.35 \pm 0.02$ & $0.30 \pm 0.04$ & $0.19 \pm 0.01$ \\
\hline $5755[\mathrm{~N}$ II $]$ & $0.08 \pm 0.00$ & $0.22 \pm 0.02$ & $0.10 \pm 0.01$ & $\ldots$ \\
\hline $5876 \mathrm{He}_{\mathrm{I}}$ & $14.62 \pm 0.22$ & $15.47 \pm 0.23$ & $11.37 \pm 0.17$ & $9.06 \pm 0.14$ \\
\hline $6300\left[\mathrm{O}_{\mathrm{I}}\right]$ & $1.68 \pm 0.03$ & $2.68 \pm 0.05$ & $3.30 \pm 0.05$ & $2.03 \pm 0.04$ \\
\hline 6312 [S III] & $2.00 \pm 0.03$ & $2.72 \pm 0.05$ & $1.51 \pm 0.03$ & $1.33 \pm 0.03$ \\
\hline 6363 [O I] & $0.55 \pm 0.01$ & $0.89 \pm 0.02$ & $1.06 \pm 0.03$ & $0.46 \pm 0.02$ \\
\hline 6548 [N II] & $0.91 \pm 0.02$ & $1.38 \pm 0.02$ & $2.59 \pm 0.04$ & $2.26 \pm 0.05$ \\
\hline $6563 \mathrm{H} \alpha$ & $281.49 \pm 4.36$ & $283.44 \pm 4.39$ & $287.72 \pm 4.50$ & $283.28 \pm 4.67$ \\
\hline 6583 [N II] & $2.71 \pm 0.04$ & $5.25 \pm 0.08$ & $8.18 \pm 0.13$ & $7.20 \pm 0.12$ \\
\hline $6678 \mathrm{He}$ I & $4.05 \pm 0.06$ & $5.15 \pm 0.08$ & $2.91 \pm 0.05$ & $2.46 \pm 0.05$ \\
\hline 6717 [S II] & $6.39 \pm 0.10$ & $15.15 \pm 0.24$ & $11.99 \pm 0.19$ & $9.56 \pm 0.16$ \\
\hline 6731 [S II] & $5.47 \pm 0.09$ & $11.68 \pm 0.18$ & $9.03 \pm 0.14$ & $7.31 \pm 0.12$ \\
\hline
\end{tabular}


Table 3. continued.

\begin{tabular}{|c|c|c|c|c|}
\hline \multirow[b]{2}{*}{ Line } & \multicolumn{4}{|c|}{$\begin{array}{c}\text { GALAXY } \\
\text { (ESO PROGRAM) }\end{array}$} \\
\hline & $\begin{array}{l}\text { NGC 5408 No.1 } \\
(081 . C-0113(\mathrm{~A}))\end{array}$ & $\begin{array}{l}\text { NGC 5408 No.2 } \\
(081 . C-0113(\mathrm{~A}))\end{array}$ & $\begin{array}{r}\text { TOL 1457-262 } \\
(081 . \mathrm{C}-0113(\mathrm{~A}))\end{array}$ & $\begin{array}{l}\text { TOL 1924-416 No.1 } \\
(081 . \mathrm{C}-0113(\mathrm{~A}))\end{array}$ \\
\hline $7065 \mathrm{He}_{\mathrm{I}}$ & $5.04 \pm 0.08$ & $2.85 \pm 0.05$ & $2.00 \pm 0.03$ & $2.20 \pm 0.04$ \\
\hline 7136 [Ar III] & $7.22 \pm 0.12$ & $9.39 \pm 0.15$ & $6.75 \pm 0.11$ & $4.76 \pm 0.08$ \\
\hline $7281 \mathrm{He}_{\mathrm{I}}$ & $0.76 \pm 0.01$ & $0.70 \pm 0.01$ & $0.51 \pm 0.02$ & $0.38 \pm 0.01$ \\
\hline 7320 [O II] & $1.68 \pm 0.03$ & $2.38 \pm 0.04$ & $1.83 \pm 0.03$ & $1.52 \pm 0.03$ \\
\hline 7330 [O II] & $1.35 \pm 0.02$ & $1.87 \pm 0.03$ & $1.61 \pm 0.03$ & $1.18 \pm 0.03$ \\
\hline 9069 [S III] & $10.58 \pm 0.19$ & $15.59 \pm 0.29$ & $13.90 \pm 0.26$ & $13.07 \pm 0.26$ \\
\hline$C(\mathrm{H} \beta)$ & 0.520 & 0.355 & 0.745 & 0.005 \\
\hline$F(\mathrm{H} \beta)^{a}$ & 1194.00 & 404.40 & 733.00 & 988.55 \\
\hline$E W(\mathrm{H} \beta) \AA$ & 309.1 & 507.8 & 95.0 & 79.4 \\
\hline$E W($ abs $) \AA$ & 0.01 & 0.55 & 0.30 & 5.65 \\
\hline \multirow[b]{2}{*}{ Line } & \multicolumn{3}{|c|}{$\begin{array}{c}\text { GaLAXY } \\
\text { (ESO PROGRAM) }\end{array}$} & \\
\hline & $\begin{array}{r}\text { TOL 1924-416 No.2 } \\
(081 . \mathrm{C}-0113(\mathrm{~A}))\end{array}$ & $\begin{array}{r}\text { NGC 6822 V } \\
(081 . C-0113(\mathrm{~A}))\end{array}$ & $\begin{array}{r}\text { PHL 293B } \\
(70 . \mathrm{B}-0717(\mathrm{~A}))\end{array}$ & \\
\hline 3727 [O II] & $113.90 \pm 1.79$ & $78.55 \pm 1.23$ & $50.21 \pm 0.84$ & \\
\hline $3750 \mathrm{H} 12$ & $5.85 \pm 0.15$ & $5.26 \pm 0.12$ & $2.95 \pm 0.16$ & \\
\hline 3771 H11 & $6.64 \pm 0.14$ & $6.07 \pm 0.11$ & $4.49 \pm 0.16$ & \\
\hline 3798 H10 & $8.07 \pm 0.15$ & $7.10 \pm 0.12$ & $5.73 \pm 0.19$ & \\
\hline $3820 \mathrm{He}_{\mathrm{I}}$ & $0.64 \pm 0.02$ & $0.92 \pm 0.02$ & $1.05 \pm 0.17$ & \\
\hline $3835 \mathrm{H} 9$ & $9.61 \pm 0.17$ & $8.96 \pm 0.15$ & $8.39 \pm 0.21$ & \\
\hline $3868[\mathrm{Ne}$ III] & $35.13 \pm 0.54$ & $35.50 \pm 0.55$ & $48.49 \pm 0.77$ & \\
\hline 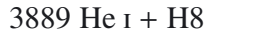 & $18.40 \pm 0.29$ & $20.55 \pm 0.32$ & $21.27 \pm 0.41$ & \\
\hline 3968 [Ne III] + H7 & $29.25 \pm 0.45$ & $28.68 \pm 0.44$ & $31.70 \pm 0.54$ & \\
\hline $4026 \mathrm{He} \mathrm{I}$ & $1.37 \pm 0.03$ & $1.60 \pm 0.02$ & $1.81 \pm 0.12$ & \\
\hline 4068 [S II] & $0.81 \pm 0.02$ & $0.48 \pm 0.01$ & $0.67 \pm 0.12$ & \\
\hline 4076 [S II] & $0.28 \pm 0.02$ & $0.14 \pm 0.01$ & $\ldots$ & \\
\hline $4101 \mathrm{H} \delta$ & $27.70 \pm 0.42$ & $26.51 \pm 0.40$ & $27.00 \pm 0.46$ & \\
\hline $4340 \mathrm{H} \gamma$ & $45.77 \pm 0.68$ & $46.14 \pm 0.68$ & $48.25 \pm 0.74$ & \\
\hline 4363 [O III] & $6.23 \pm 0.09$ & $5.18 \pm 0.08$ & $14.55 \pm 0.27$ & \\
\hline $4387 \mathrm{He}$ I & $0.32 \pm 0.01$ & $0.49 \pm 0.01$ & $\ldots$ & \\
\hline $4471 \mathrm{He} \mathrm{I}$ & $3.23 \pm 0.05$ & $3.54 \pm 0.05$ & $3.75 \pm 0.10$ & \\
\hline 4658 [Fe III] & $0.88 \pm 0.02$ & $0.19 \pm 0.01$ & $\ldots$ & \\
\hline 4686 Не II & $0.93 \pm 0.03$ & $\ldots$ & $\ldots$ & \\
\hline $4711[\mathrm{Ar}$ IV $]+\mathrm{He}_{\mathrm{I}}$ & $0.92 \pm 0.02$ & $0.71 \pm 0.01$ & $\ldots$ & \\
\hline $4740[\mathrm{Ar} \mathrm{IV}]$ & $0.43 \pm 0.02$ & $0.20 \pm 0.01$ & $\ldots$ & \\
\hline $4861 \mathrm{H} \beta$ & $100.00 \pm 1.46$ & $100.00 \pm 1.45$ & $100.00 \pm 1.44$ & \\
\hline $4921 \mathrm{He} \mathrm{I}$ & $0.86 \pm 0.02$ & $1.04 \pm 0.02$ & $0.93 \pm 0.04$ & \\
\hline 4959 [O III] & $173.25 \pm 2.52$ & $186.37 \pm 2.69$ & $195.13 \pm 2.79$ & \\
\hline 5007 [O III] & $531.57 \pm 7.73$ & $530.38 \pm 6.60$ & $584.35 \pm 8.33$ & \\
\hline $5015 \mathrm{He}_{\mathrm{I}}$ & $1.63 \pm 0.03$ & $2.27 \pm 0.03$ & $2.11 \pm 0.04$ & \\
\hline 5199 [N I] & $0.53 \pm 0.02$ & $0.13 \pm 0.00$ & ;. & \\
\hline 5518 [Cl III] & $0.28 \pm 0.01$ & $0.42 \pm 0.01$ & $0.26 \pm 0.03$ & \\
\hline $5538[\mathrm{Cl} \mathrm{III]}$ & $0.17 \pm 0.01$ & $0.29 \pm 0.01$ & $\ldots$ & \\
\hline 5755 [N II] & $\ldots$ & $0.10 \pm 0.01$ & $\ldots$ & \\
\hline $5876 \mathrm{He}_{\mathrm{I}}$ & $10.67 \pm 0.16$ & $12.32 \pm 0.18$ & $10.07 \pm 0.17$ & \\
\hline $6300\left[\mathrm{O}_{\mathrm{I}}\right]$ & $2.81 \pm 0.05$ & $0.74 \pm 0.01$ & $1.19 \pm 0.05$ & \\
\hline 6312 [S III] & $1.71 \pm 0.03$ & $2.27 \pm 0.04$ & $1.60 \pm 0.05$ & \\
\hline $6363\left[\mathrm{O}_{\mathrm{I}}\right]$ & $0.83 \pm 0.02$ & $0.23 \pm 0.01$ & $\ldots$ & \\
\hline 6548 [N II] & $1.88 \pm 0.04$ & $1.50 \pm 0.02$ & $\ldots$ & \\
\hline $6563 \mathrm{H} \alpha$ & $284.01 \pm 4.48$ & $287.13 \pm 4.50$ & $277.28 \pm 4.31$ & \\
\hline 6583 [N II] & $5.54 \pm 0.09$ & $4.49 \pm 0.07$ & $1.82 \pm 0.06$ & \\
\hline $6678 \mathrm{He} \mathrm{I}$ & $2.92 \pm 0.05$ & $3.59 \pm 0.06$ & $2.76 \pm 0.05$ & \\
\hline 6717 [S II] & $10.88 \pm 0.17$ & $6.03 \pm 0.10$ & $5.04 \pm 0.11$ & \\
\hline $6731[\mathrm{~S} \mathrm{II}]$ & $8.59 \pm 0.14$ & $4.62 \pm 0.07$ & $3.68 \pm 0.08$ & \\
\hline
\end{tabular}


Table 3. continued.

\begin{tabular}{|c|c|c|c|}
\hline \multirow[b]{2}{*}{ Line } & \multicolumn{3}{|c|}{$\begin{array}{c}\text { GALAXY } \\
\text { (ESO PROGRAM) }\end{array}$} \\
\hline & $\begin{array}{r}\text { TOL 1924-416 No.2 } \\
(081 . \mathrm{C}-0113(\mathrm{~A}))\end{array}$ & $\begin{array}{r}\text { NGC 6822 V } \\
(081 . C-0113(\mathrm{~A}))\end{array}$ & $\begin{array}{r}\text { PHL 293B } \\
(70 . \mathrm{B}-0717(\mathrm{~A}))\end{array}$ \\
\hline $7065 \mathrm{He} \mathrm{I}$ & $2.58 \pm 0.04$ & $2.69 \pm 0.04$ & $\ldots$ \\
\hline 7136 [Ar III] & $6.20 \pm 0.10$ & $10.57 \pm 0.17$ & $\ldots$ \\
\hline $7281 \mathrm{He}_{\mathrm{I}}$ & $0.49 \pm 0.01$ & $0.66 \pm 0.01$ & $\ldots$ \\
\hline 7320 [O II] & $1.96 \pm 0.03$ & $1.62 \pm 0.03$ & $\ldots$ \\
\hline 7330 [O II] & $1.67 \pm 0.03$ & $1.26 \pm 0.02$ & $\ldots$ \\
\hline 9069 [S III] & $13.98 \pm 0.26$ & $25.60 \pm 0.48$ & $\ldots$ \\
\hline$C(\mathrm{H} \beta)$ & 0.090 & 0.470 & 0.080 \\
\hline$F(\mathrm{H} \beta)^{a}$ & 960.95 & 1643.00 & 71.45 \\
\hline$E W(\mathrm{H} \beta) \AA$ & 155.3 & 327.3 & 37.0 \\
\hline$E W(\mathrm{abs}) \AA$ & 3.65 & 4.20 & 0.05 \\
\hline
\end{tabular}

Notes. ${ }^{(a)}$ In units $10^{-16} \mathrm{erg} \mathrm{s}^{-1} \mathrm{~cm}^{-2}$.

Table 4. Extinction-corrected emission line fluxes (low-resolution FORS observations).

\begin{tabular}{|c|c|c|c|c|}
\hline \multirow[b]{2}{*}{ Line } & \multicolumn{4}{|c|}{$\begin{array}{c}\text { GaLAXY } \\
\text { (ESO PROGRAM) }\end{array}$} \\
\hline & $\begin{array}{r}\text { NGC 346A No.1 } \\
(69 . \mathrm{C}-0203(\mathrm{~A}))\end{array}$ & $\begin{array}{r}\text { NGC 346A No. } 2 \\
(69 . C-0203(A))\end{array}$ & $\begin{array}{r}\text { NGC 346A No.3 } \\
(69 . C-0203(A))\end{array}$ & $\begin{array}{r}\text { NGC 346B No.1 } \\
(69 . \mathrm{C}-0203(\mathrm{~A}))\end{array}$ \\
\hline $3727[\mathrm{O}$ II] & $75.53 \pm 1.27$ & $94.10 \pm 1.61$ & $105.08 \pm 2.08$ & $133.94 \pm 2.33$ \\
\hline $3750 \mathrm{H} 12$ & $4.80 \pm 0.43$ & $4.62 \pm 0.58$ & $\ldots$ & $6.11 \pm 1.15$ \\
\hline $3771 \mathrm{H} 11$ & $5.45 \pm 0.37$ & $5.30 \pm 0.44$ & $\ldots$ & $6.83 \pm 0.80$ \\
\hline 3798 H10 & $6.14 \pm 0.31$ & $6.58 \pm 0.42$ & $\ldots$ & $7.35 \pm 0.70$ \\
\hline $3820 \mathrm{He}_{\mathrm{I}}$ & $0.90 \pm 0.14$ & $1.70 \pm 0.28$ & $\ldots$ & $\ldots$ \\
\hline $3835 \mathrm{H} 9$ & $8.68 \pm 0.30$ & $8.13 \pm 0.40$ & $\ldots$ & $11.11 \pm 0.60$ \\
\hline 3868 [Ne III] & $36.74 \pm 0.58$ & $41.12 \pm 0.66$ & $38.28 \pm 0.75$ & $35.57 \pm 0.62$ \\
\hline 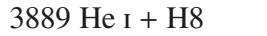 & $20.65 \pm 0.39$ & $20.43 \pm 0.47$ & $20.28 \pm 0.67$ & $21.50 \pm 0.55$ \\
\hline 3968 [Ne III] + H7 & $27.79 \pm 0.47$ & $29.72 \pm 0.57$ & $27.00 \pm 0.62$ & $28.32 \pm 0.61$ \\
\hline 4026 He I & $1.64 \pm 0.07$ & $1.93 \pm 0.10$ & 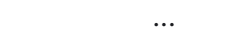 & $2.03 \pm 0.14$ \\
\hline $4101 \mathrm{H} \delta$ & $26.63 \pm 0.45$ & $26.35 \pm 0.51$ & $26.63 \pm 0.55$ & $27.22 \pm 0.56$ \\
\hline $4340 \mathrm{H} \gamma$ & $46.69 \pm 0.71$ & $47.94 \pm 0.75$ & $46.20 \pm 0.75$ & $46.14 \pm 0.77$ \\
\hline $4363[\mathrm{O} \mathrm{III]}$ & $6.52 \pm 0.11$ & $6.81 \pm 0.12$ & $5.91 \pm 0.18$ & $5.80 \pm 0.14$ \\
\hline $4387 \mathrm{He}_{\mathrm{I}}$ & $0.36 \pm 0.04$ & $\ldots$ & $\ldots$ & $\ldots$ \\
\hline $4471 \mathrm{He}$ I & $3.85 \pm 0.07$ & $3.82 \pm 0.08$ & $2.62 \pm 0.15$ & $4.02 \pm 0.11$ \\
\hline $4711[$ Ar Iv $]+\mathrm{He} \mathrm{I}$ & $0.82 \pm 0.01$ & $1.35 \pm 0.05$ & $\ldots$ & $\ldots$ \\
\hline $4740[\mathrm{Ar} \mathrm{IV}]$ & $0.31 \pm 0.00$ & $0.70 \pm 0.04$ & $\ldots$ & $\ldots$ \\
\hline $4861 \mathrm{H} \beta$ & $100.00 \pm 1.45$ & $100.00 \pm 1.45$ & $100.00 \pm 1.50$ & $100.00 \pm 1.50$ \\
\hline $4921 \mathrm{He}_{\mathrm{I}}$ & $1.04 \pm 0.04$ & $1.10 \pm 0.05$ & $\ldots$ & $1.06 \pm 0.07$ \\
\hline 4959 [O III] & $181.36 \pm 2.61$ & $179.46 \pm 2.56$ & $166.32 \pm 2.47$ & $158.42 \pm 2.31$ \\
\hline 5007 [O III] & $551.58 \pm 7.94$ & $525.90 \pm 7.50$ & $488.05 \pm 7.23$ & $481.13 \pm 7.01$ \\
\hline $5876 \mathrm{He}$ I & $11.15 \pm 0.17$ & $11.31 \pm 0.17$ & $10.52 \pm 0.18$ & $10.99 \pm 0.18$ \\
\hline 6312 [S III] & $1.99 \pm 0.04$ & $1.44 \pm 0.04$ & $1.54 \pm 0.06$ & $1.76 \pm 0.06$ \\
\hline 6548 [N II] & $0.95 \pm 0.03$ & $1.22 \pm 0.04$ & $1.37 \pm 0.07$ & $1.66 \pm 0.05$ \\
\hline $6563 \mathrm{H} \alpha$ & $283.77 \pm 4.43$ & $283.34 \pm 4.39$ & $283.74 \pm 4.56$ & $283.61 \pm 4.49$ \\
\hline $6583[\mathrm{~N} \mathrm{II}]$ & $2.05 \pm 0.04$ & $3.84 \pm 0.07$ & $4.07 \pm 0.10$ & $4.95 \pm 0.10$ \\
\hline $6678 \mathrm{He}$ I & $3.00 \pm 0.06$ & $2.99 \pm 0.06$ & $2.95 \pm 0.09$ & $3.01 \pm 0.07$ \\
\hline 6717 [S II] & $3.98 \pm 0.07$ & $7.50 \pm 0.12$ & $7.91 \pm 0.15$ & $8.92 \pm 0.16$ \\
\hline 6731 [S II] & $2.78 \pm 0.05$ & $5.48 \pm 0.09$ & $5.89 \pm 0.13$ & $6.43 \pm 0.12$ \\
\hline $7065 \mathrm{He}$ I & $2.04 \pm 0.04$ & $2.00 \pm 0.05$ & $1.91 \pm 0.08$ & $2.05 \pm 0.07$ \\
\hline 7136 [Ar III] & $8.18 \pm 0.14$ & $7.11 \pm 0.12$ & $7.79 \pm 0.16$ & $8.11 \pm 0.15$ \\
\hline$C(\mathrm{H} \beta)$ & 0.060 & 0.105 & 0.065 & 0.060 \\
\hline$F(\mathrm{H} \beta)^{a}$ & 602.50 & 414.40 & 339.80 & 470.50 \\
\hline$E W(\mathrm{H} \beta) \AA$ & 281.3 & 292.4 & 55.9 & 259.8 \\
\hline$E W($ abs $) \AA$ & 3.55 & 0.50 & 2.05 & 5.10 \\
\hline \multirow[b]{2}{*}{ Line } & \multicolumn{4}{|c|}{$\begin{array}{c}\text { GalaXy } \\
\text { (ESO PROGRAM) }\end{array}$} \\
\hline & $\begin{array}{r}\text { NGC 346B No.2 } \\
(69 . C-0203(A))\end{array}$ & $\begin{array}{r}\text { NGC 346D No.1 } \\
(69 . C-0203(A))\end{array}$ & $\begin{array}{r}\text { NGC 346D No.2 } \\
(69 . C-0203(A))\end{array}$ & $\begin{array}{r}\text { NGC 346D No.3 } \\
(69 . C-0203(A))\end{array}$ \\
\hline 3727 [O II] & $90.86 \pm 1.59$ & $150.56 \pm 2.49$ & $76.18 \pm 1.39$ & $99.17 \pm 1.78$ \\
\hline $3750 \mathrm{H} 12$ & $4.60 \pm 0.54$ & $4.56 \pm 0.55$ & $\ldots$ & $\ldots$ \\
\hline 3771 H11 & $4.54 \pm 0.44$ & $5.92 \pm 0.54$ & $\ldots$ & $5.78 \pm 0.66$ \\
\hline
\end{tabular}


Table 4. continued.

\begin{tabular}{|c|c|c|c|c|}
\hline \multirow[b]{2}{*}{ Line } & \multicolumn{4}{|c|}{$\begin{array}{c}\text { GaLaXy } \\
\text { (ESO PROGRAM) }\end{array}$} \\
\hline & $\begin{array}{r}\text { NGC 346B No.2 } \\
(69 . \mathrm{C}-0203(\mathrm{~A}))\end{array}$ & $\begin{array}{r}\text { NGC 346D No.1 } \\
(69 . C-0203(A))\end{array}$ & $\begin{array}{r}\text { NGC 346D No.2 } \\
(69 . \mathrm{C}-0203(\mathrm{~A}))\end{array}$ & $\begin{array}{r}\text { NGC 346D No.3 } \\
(69 . \mathrm{C}-0203(\mathrm{~A}))\end{array}$ \\
\hline $3798 \mathrm{H} 10$ & $5.83 \pm 0.41$ & $6.25 \pm 0.54$ & $5.56 \pm 0.39$ & $6.92 \pm 0.54$ \\
\hline 3835 H9 & $8.83 \pm 0.39$ & $8.31 \pm 0.51$ & $7.87 \pm 0.35$ & $8.88 \pm 0.47$ \\
\hline $3868[\mathrm{Ne}$ III] & $39.24 \pm 0.64$ & $35.17 \pm 0.59$ & $38.66 \pm 0.65$ & $36.30 \pm 0.62$ \\
\hline $3889 \mathrm{He}_{\mathrm{I}}+\mathrm{H} 8$ & $19.57 \pm 0.43$ & $19.71 \pm 0.58$ & $20.44 \pm 0.43$ & $22.41 \pm 0.52$ \\
\hline 3968 [Ne III] + H7 & $29.21 \pm 0.53$ & $27.71 \pm 0.65$ & $28.25 \pm 0.52$ & $28.89 \pm 0.58$ \\
\hline $4026 \mathrm{He}_{\mathrm{I}}$ & $1.41 \pm 0.10$ & $1.69 \pm 0.13$ & $1.45 \pm 0.09$ & $2.00 \pm 0.14$ \\
\hline $4101 \mathrm{H} \delta$ & $26.98 \pm 0.47$ & $26.03 \pm 0.61$ & $26.15 \pm 0.49$ & $26.41 \pm 0.54$ \\
\hline $4340 \mathrm{H} \gamma$ & $47.99 \pm 0.73$ & $46.94 \pm 0.80$ & $47.24 \pm 0.74$ & $46.65 \pm 0.76$ \\
\hline 4363 [O III] & $6.82 \pm 0.13$ & $6.84 \pm 0.14$ & $7.59 \pm 0.15$ & $6.23 \pm 0.13$ \\
\hline $4471 \mathrm{He}_{\mathrm{I}}$ & $3.85 \pm 0.10$ & $3.73 \pm 0.10$ & $3.86 \pm 0.09$ & $3.96 \pm 0.10$ \\
\hline $4711[\mathrm{Ar} \mathrm{IV}]+\mathrm{He}_{\mathrm{I}}$ & $1.33 \pm 0.06$ & $\ldots$ & $\ldots$ & $0.89 \pm 0.06$ \\
\hline 4740 [Ar Iv] & $0.66 \pm 0.06$ & $\ldots$ & $\ldots$ & $0.40 \pm 0.06$ \\
\hline $4861 \mathrm{H} \beta$ & $100.00 \pm 1.45$ & $100.00 \pm 1.48$ & $100.00 \pm 1.46$ & $100.00 \pm 1.48$ \\
\hline $4921 \mathrm{He}$ I & $0.93 \pm 0.05$ & $0.96 \pm 0.05$ & $0.94 \pm 0.05$ & $1.11 \pm 0.06$ \\
\hline 4959 [O III] & $176.70 \pm 2.54$ & $159.00 \pm 2.28$ & $174.45 \pm 2.52$ & $160.68 \pm 2.34$ \\
\hline $5007[\mathrm{O} \mathrm{III}]$ & $517.04 \pm 7.41$ & $473.60 \pm 6.78$ & $517.51 \pm 7.46$ & $476.84 \pm 6.92$ \\
\hline $5876 \mathrm{He}_{\mathrm{I}}$ & $11.74 \pm 0.18$ & $10.41 \pm 0.16$ & $10.72 \pm 0.17$ & $11.80 \pm 0.19$ \\
\hline 6312 [S III] & $1.49 \pm 0.05$ & $1.69 \pm 0.04$ & $1.92 \pm 0.05$ & $2.20 \pm 0.06$ \\
\hline 6548 [N II] & $0.93 \pm 0.04$ & $2.50 \pm 0.06$ & $1.18 \pm 0.04$ & $1.53 \pm 0.05$ \\
\hline $6563 \mathrm{H} \alpha$ & $283.10 \pm 4.40$ & $282.32 \pm 4.39$ & $282.08 \pm 4.42$ & $282.86 \pm 4.46$ \\
\hline $6583[\mathrm{~N}$ II] & $2.68 \pm 0.06$ & $6.14 \pm 0.11$ & $2.58 \pm 0.06$ & $4.34 \pm 0.08$ \\
\hline $6678 \mathrm{He}_{\mathrm{I}}$ & $3.04 \pm 0.07$ & $2.83 \pm 0.06$ & $2.85 \pm 0.06$ & $3.01 \pm 0.07$ \\
\hline 6717 [S II] & $4.01 \pm 0.08$ & $11.80 \pm 0.19$ & $6.02 \pm 0.11$ & $7.77 \pm 0.13$ \\
\hline 6731 [S II] & $2.88 \pm 0.06$ & $8.37 \pm 0.14$ & $4.13 \pm 0.08$ & $5.87 \pm 0.11$ \\
\hline $7065 \mathrm{He}_{\mathrm{I}}$ & $2.13 \pm 0.05$ & $1.96 \pm 0.05$ & $2.21 \pm 0.06$ & $2.30 \pm 0.06$ \\
\hline 7136 [Ar III] & $7.23 \pm 0.13$ & $7.49 \pm 0.13$ & $7.67 \pm 0.14$ & $8.72 \pm 0.16$ \\
\hline$C(\mathrm{H} \beta)$ & 0.075 & 0.080 & 0.055 & 0.025 \\
\hline$F(\mathrm{H} \beta)^{a}$ & 534.30 & 531.40 & 350.20 & 403.30 \\
\hline$E W(\mathrm{H} \beta) \AA$ & 173.0 & 273.2 & 274.9 & 309.8 \\
\hline$E W(\mathrm{abs}) \AA$ & 0.70 & 1.35 & 2.70 & 5.65 \\
\hline \multirow[b]{2}{*}{ Line } & \multicolumn{4}{|c|}{$\begin{array}{c}\text { GaLAXY } \\
\text { (ESO PROGRAM) }\end{array}$} \\
\hline & $\begin{array}{r}\text { NGC 346D No.4 } \\
(69 . \mathrm{C}-0203(\mathrm{~A}))\end{array}$ & $\begin{array}{r}\text { NGC 456A No.1 } \\
(69 . C-0203(A))\end{array}$ & $\begin{array}{r}\text { NGC 456A No.2 } \\
(69 . C-0203(A))\end{array}$ & $\begin{array}{r}\text { NGC 456A No.3 } \\
(69 . C-0203(A))\end{array}$ \\
\hline 3727 [O II] & $100.98 \pm 1.77$ & $223.88 \pm 3.61$ & $148.34 \pm 2.64$ & $170.44 \pm 2.90$ \\
\hline $3750 \mathrm{H} 12$ & $5.50 \pm 1.00$ & $4.15 \pm 0.43$ & $3.50 \pm 0.72$ & $6.36 \pm 0.63$ \\
\hline $3771 \mathrm{H} 11$ & $6.23 \pm 0.70$ & $5.08 \pm 0.42$ & $4.09 \pm 0.67$ & $5.78 \pm 0.52$ \\
\hline 3798 H10 & $7.16 \pm 0.57$ & $5.78 \pm 0.38$ & $5.30 \pm 0.60$ & $6.40 \pm 0.47$ \\
\hline $3820 \mathrm{He}$ I & $\ldots$ & $\ldots$ & $\ldots$ & $1.01 \pm 0.18$ \\
\hline $3835 \mathrm{H} 9$ & $8.88 \pm 0.47$ & $8.88 \pm 0.36$ & $7.76 \pm 0.60$ & $8.60 \pm 0.42$ \\
\hline 3868 [Ne III] & $37.22 \pm 0.63$ & $24.59 \pm 0.43$ & $30.09 \pm 0.60$ & $25.09 \pm 0.47$ \\
\hline $3889 \mathrm{He}_{\mathrm{I}}+\mathrm{H} 8$ & $21.03 \pm 0.50$ & $20.00 \pm 0.47$ & $20.42 \pm 0.64$ & $20.76 \pm 0.50$ \\
\hline $3968[\mathrm{Ne}$ III] + H7 & $27.92 \pm 0.55$ & $25.75 \pm 0.52$ & $27.39 \pm 0.67$ & $25.94 \pm 0.53$ \\
\hline $4026 \mathrm{He}$ I & $1.63 \pm 0.11$ & $2.10 \pm 0.09$ & $1.78 \pm 0.16$ & $1.97 \pm 0.13$ \\
\hline 4068 [S II] & $\ldots$ & $1.52 \pm 0.07$ & $\ldots$ & $0.90 \pm 0.10$ \\
\hline $4101 \mathrm{H} \delta$ & $25.68 \pm 0.51$ & $26.03 \pm 0.49$ & $25.62 \pm 0.60$ & $25.92 \pm 0.51$ \\
\hline $4340 \mathrm{H} \gamma$ & $45.80 \pm 0.74$ & $46.91 \pm 0.75$ & $47.17 \pm 0.79$ & $46.17 \pm 0.74$ \\
\hline $4363[\mathrm{O} \mathrm{III}]$ & $6.58 \pm 0.13$ & $4.53 \pm 0.09$ & $5.07 \pm 0.14$ & $4.56 \pm 0.11$ \\
\hline $4387 \mathrm{He}_{\mathrm{I}}$ & $\ldots$ & $0.51 \pm 0.04$ & 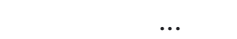 & $0.45 \pm 0.05$ \\
\hline $4471 \mathrm{He}_{\mathrm{I}}$ & $3.65 \pm 0.09$ & $3.99 \pm 0.08$ & $3.68 \pm 0.12$ & $3.99 \pm 0.10$ \\
\hline 4658 [Fe III] & $\ldots$ & $0.47 \pm 0.05$ & $\ldots$ & $0.32 \pm 0.04$ \\
\hline $4711[\mathrm{Ar} \mathrm{IV}]+\mathrm{He}_{\mathrm{I}}$ & $0.74 \pm 0.06$ & $\ldots$ & $\ldots$ & $\ldots$ \\
\hline 4740 [Ar IV] & $0.54 \pm 0.06$ & $\ldots$ & $\ldots$ & $\ldots$ \\
\hline $4861 \mathrm{H} \beta$ & $100.00 \pm 1.48$ & $100.00 \pm 1.46$ & $100.00 \pm 1.48$ & $100.00 \pm 1.48$ \\
\hline $4921 \mathrm{He}_{\mathrm{I}}$ & $1.03 \pm 0.05$ & $1.15 \pm 0.04$ & $1.16 \pm 0.08$ & $1.26 \pm 0.06$ \\
\hline 4959 [O III] & $159.28 \pm 2.32$ & $129.01 \pm 1.86$ & $146.99 \pm 2.14$ & $136.81 \pm 1.99$ \\
\hline 4988 [Fe III] & $\ldots$ & $0.39 \pm 0.04$ & $\ldots$ & $0.35 \pm 0.01$ \\
\hline 5007 [O III] & $475.79 \pm 6.92$ & $379.19 \pm 5.44$ & $426.61 \pm 6.17$ & $395.52 \pm 5.73$ \\
\hline $5518[\mathrm{Cl} \mathrm{III}]$ & $\ldots$ & $0.48 \pm 0.03$ & $0.45 \pm 0.06$ & $0.27 \pm 0.03$ \\
\hline 5538 [Cl III] & $\ldots$ & $0.32 \pm 0.03$ & $0.27 \pm 0.05$ & $0.26 \pm 0.03$ \\
\hline $5755[\mathrm{~N}$ II $]$ & $\ldots$ & $0.17 \pm 0.00$ & $\ldots$ & $\ldots$ \\
\hline $5876 \mathrm{He}_{\mathrm{I}}$ & $10.96 \pm 0.18$ & $11.44 \pm 0.18$ & $11.20 \pm 0.19$ & $11.36 \pm 0.18$ \\
\hline
\end{tabular}


Table 4. continued.

\begin{tabular}{|c|c|c|c|c|}
\hline \multirow[b]{2}{*}{ Line } & \multicolumn{4}{|c|}{$\begin{array}{c}\text { GALAXY } \\
\text { (ESO PROGRAM) }\end{array}$} \\
\hline & $\begin{array}{r}\text { NGC 346D No.4 } \\
(69 . C-0203(A))\end{array}$ & $\begin{array}{r}\text { NGC 456A No.1 } \\
(69 . \mathrm{C}-0203(\mathrm{~A}))\end{array}$ & $\begin{array}{r}\text { NGC 456A No.2 } \\
(69 . C-0203(A))\end{array}$ & $\begin{array}{r}\text { NGC 456A No.3 } \\
(69 . C-0203(A))\end{array}$ \\
\hline $6312[\mathrm{~S}$ III] & $2.00 \pm 0.05$ & $1.87 \pm 0.04$ & $1.55 \pm 0.06$ & $1.83 \pm 0.05$ \\
\hline $6548[\mathrm{~N} \mathrm{II}]$ & $1.55 \pm 0.05$ & $2.82 \pm 0.06$ & $1.29 \pm 0.05$ & $1.29 \pm 0.04$ \\
\hline $6563 \mathrm{H} \alpha$ & $282.27 \pm 4.45$ & $284.35 \pm 4.43$ & $284.30 \pm 4.46$ & $284.51 \pm 4.47$ \\
\hline 6583 [N II] & $3.76 \pm 0.08$ & $8.04 \pm 0.14$ & $5.30 \pm 0.11$ & $4.89 \pm 0.09$ \\
\hline $6678 \mathrm{He} \mathrm{I}$ & $2.98 \pm 0.07$ & $3.09 \pm 0.06$ & $3.09 \pm 0.08$ & $3.08 \pm 0.07$ \\
\hline 6717 [S II] & $8.00 \pm 0.14$ & $9.76 \pm 0.16$ & $7.28 \pm 0.13$ & $6.59 \pm 0.12$ \\
\hline 6731 [S II] & $5.78 \pm 0.10$ & $8.14 \pm 0.14$ & $5.29 \pm 0.10$ & $5.00 \pm 0.10$ \\
\hline $7065 \mathrm{He} \mathrm{I}$ & $2.13 \pm 0.06$ & $2.65 \pm 0.06$ & $2.18 \pm 0.07$ & $2.37 \pm 0.06$ \\
\hline 7136 [Ar III] & $7.73 \pm 0.14$ & $8.20 \pm 0.14$ & $7.90 \pm 0.15$ & $7.94 \pm 0.14$ \\
\hline $7320[\mathrm{O}$ II $]$ & $\ldots$ & $3.96 \pm 0.08$ & $\ldots$ & $\ldots$ \\
\hline 7330 [O II] & $\ldots$ & $3.49 \pm 0.07$ & $\ldots$ & $\ldots$ \\
\hline$C(\mathrm{H} \beta)$ & 0.005 & 0.190 & 0.155 & 0.160 \\
\hline$F(\mathrm{H} \beta)^{a}$ & 404.70 & 371.90 & 214.70 & 246.60 \\
\hline$E W(\mathrm{H} \beta) \AA$ & 265.4 & 654.3 & 254.9 & 575.4 \\
\hline$E W($ abs $) \AA$ & 5.20 & 0.80 & 0.50 & 3.15 \\
\hline \multirow[b]{2}{*}{ Line } & \multicolumn{4}{|c|}{$\begin{array}{l}\text { GalaXY } \\
\text { ESO PROGRAM) }\end{array}$} \\
\hline & $\begin{array}{r}\text { UM } 420 \text { No.2 } \\
(69 . \mathrm{C}-0203(\mathrm{~A}))\end{array}$ & $\begin{array}{r}\text { CAM 0357-3915 } \\
(69 . \mathrm{C}-0203(\mathrm{~A})) \\
\end{array}$ & $\begin{array}{l}\text { TOL 0513-393 } \\
(69 . C-0203(A)) \\
\end{array}$ & $\begin{array}{r}\text { NGC 6822V No.1 } \\
(69 . C-0203(\mathrm{~A})) \\
\end{array}$ \\
\hline 3727 [O II] & $218.12 \pm 4.97$ & $82.50 \pm 1.50$ & $47.81 \pm 0.83$ & $90.86 \pm 1.97$ \\
\hline 3750 H12 & $\ldots$ & $3.57 \pm 0.59$ & $3.83 \pm 0.35$ & $3.24 \pm 0.80$ \\
\hline 3771 H11 & $\ldots$ & $3.71 \pm 0.50$ & $4.52 \pm 0.34$ & $4.16 \pm 0.74$ \\
\hline 3798 H10 & $\ldots$ & $5.11 \pm 0.52$ & $5.25 \pm 0.31$ & $4.86 \pm 0.66$ \\
\hline $3820 \mathrm{He} \mathrm{I}$ & $\ldots$ & $1.11 \pm 0.21$ & $0.78 \pm 0.16$ & $1.48 \pm 0.33$ \\
\hline 3835 H9 & $6.89 \pm 1.23$ & $6.63 \pm 0.49$ & $6.67 \pm 0.28$ & $7.36 \pm 0.67$ \\
\hline 3868 [Ne III] & $48.29 \pm 1.58$ & $51.52 \pm 0.96$ & $43.14 \pm 0.72$ & $41.70 \pm 0.82$ \\
\hline $3889 \mathrm{He}_{\mathrm{I}}+\mathrm{H} 8$ & $18.37 \pm 1.35$ & $18.51 \pm 0.58$ & $15.34 \pm 0.34$ & $18.54 \pm 0.71$ \\
\hline $3968[\mathrm{Ne}$ III] + H7 & $30.32 \pm 1.40$ & $31.45 \pm 0.70$ & $27.94 \pm 0.50$ & $28.12 \pm 0.75$ \\
\hline $4026 \mathrm{He} \mathrm{I}$ & $\ldots$ & $1.31 \pm 0.20$ & $1.41 \pm 0.10$ & $1.63 \pm 0.20$ \\
\hline 4068 [S II] & $\ldots$ & $\ldots$ & $0.90 \pm 0.08$ & $0.74 \pm 0.17$ \\
\hline $4101 \mathrm{H} \delta$ & $26.66 \pm 1.17$ & $25.47 \pm 0.59$ & $22.64 \pm 0.42$ & $25.93 \pm 0.65$ \\
\hline $4340 \mathrm{H} \gamma$ & $49.21 \pm 1.37$ & $48.90 \pm 0.86$ & $43.15 \pm 0.68$ & $46.76 \pm 0.83$ \\
\hline 4363 [O III] & $6.51 \pm 0.62$ & $13.61 \pm 0.33$ & $14.76 \pm 0.25$ & $5.80 \pm 0.18$ \\
\hline $4387 \mathrm{He}_{\mathrm{I}}$ & $\ldots$ & $0.58 \pm 0.17$ & $0.48 \pm 0.08$ & $0.64 \pm 0.13$ \\
\hline $4471 \mathrm{He}_{\mathrm{I}}$ & $5.09 \pm 0.58$ & $3.68 \pm 0.18$ & $3.71 \pm 0.10$ & $4.15 \pm 0.15$ \\
\hline 4658 [Fe III] & $\ldots$ & $0.76 \pm 0.12$ & $0.36 \pm 0.05$ & $\ldots$ \\
\hline $4686 \mathrm{He}$ II & $\ldots$ & $1.77 \pm 0.14$ & $1.02 \pm 0.06$ & $\ldots$ \\
\hline $4711[\mathrm{Ar} \mathrm{IV}]+\mathrm{He}_{\mathrm{I}}$ & $\ldots$ & $1.71 \pm 0.16$ & $2.40 \pm 0.07$ & $0.90 \pm 0.09$ \\
\hline 4740 [Ar IV] & $\ldots$ & $1.35 \pm 0.15$ & $1.50 \pm 0.06$ & $0.37 \pm 0.06$ \\
\hline $4861 \mathrm{H} \beta$ & $100.00 \pm 2.03$ & $100.00 \pm 1.54$ & $100.00 \pm 1.48$ & $100.00 \pm 1.51$ \\
\hline $4921 \mathrm{He}_{\mathrm{I}}$ & $\ldots$ & $0.70 \pm 0.10$ & $1.20 \pm 0.06$ & $1.08 \pm 0.09$ \\
\hline 4959 [O III] & $180.80 \pm 3.35$ & $221.05 \pm 3.32$ & $246.88 \pm 3.62$ & $184.23 \pm 2.71$ \\
\hline 4988 [Fe III] & $\ldots$ & $1.18 \pm 0.13$ & $\ldots$ & $\ldots$ \\
\hline 5007 [O III] & $547.22 \pm 9.67$ & $664.73 \pm 9.88$ & $747.31 \pm 10.93$ & $567.64 \pm 8.30$ \\
\hline 5518 [Cl III] & $\ldots$ & $0.33 \pm 0.10$ & $0.32 \pm 0.05$ & $0.40 \pm 0.07$ \\
\hline 5538 [Cl III] & $\ldots$ & $0.38 \pm 0.09$ & $0.19 \pm 0.05$ & $0.38 \pm 0.06$ \\
\hline $5876 \mathrm{He} \mathrm{I}$ & $10.25 \pm 0.42$ & $10.39 \pm 0.22$ & $11.37 \pm 0.19$ & $11.84 \pm 0.20$ \\
\hline 6300 [O I] & $4.82 \pm 0.32$ & $2.29 \pm 0.11$ & $2.00 \pm 0.05$ & $\ldots$ \\
\hline $6312[\mathrm{~S} \mathrm{III}]$ & $\ldots$ & $1.30 \pm 0.09$ & $1.23 \pm 0.05$ & $1.91 \pm 0.06$ \\
\hline $6363\left[\mathrm{O}_{\mathrm{I}}\right]$ & $1.90 \pm 0.24$ & $0.51 \pm 0.07$ & $0.65 \pm 0.04$ & \\
\hline 6548 [N II] & $4.70 \pm 0.27$ & 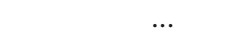 & $\ldots$ & $0.97 \pm 0.05$ \\
\hline $6563 \mathrm{H} \alpha$ & $285.07 \pm 5.52$ & $280.00 \pm 4.54$ & $280.98 \pm 4.46$ & $287.27 \pm 4.56$ \\
\hline 6583 [N II] & $11.04 \pm 0.41$ & $4.61 \pm 0.14$ & $3.27 \pm 0.07$ & $3.25 \pm 0.08$ \\
\hline $6678 \mathrm{He} \mathrm{I}$ & $3.27 \pm 0.25$ & $2.83 \pm 0.12$ & $2.93 \pm 0.07$ & $3.18 \pm 0.07$ \\
\hline 6717 [S II] & $20.03 \pm 0.55$ & $5.93 \pm 0.16$ & $5.13 \pm 0.10$ & $4.26 \pm 0.09$ \\
\hline 6731 [S II] & $15.10 \pm 0.47$ & $4.93 \pm 0.14$ & $4.14 \pm 0.08$ & $3.17 \pm 0.07$ \\
\hline 7065 He I & $3.05 \pm 0.29$ & $3.17 \pm 0.12$ & $4.00 \pm 0.08$ & $2.55 \pm 0.07$ \\
\hline 7136 [Ar III] & $6.84 \pm 0.40$ & $3.73 \pm 0.13$ & $4.36 \pm 0.09$ & $9.50 \pm 0.17$ \\
\hline $7281 \mathrm{He} \mathrm{I}^{2}$ & $\ldots$ & $\ldots$ & $\ldots$ & $0.63 \pm 0.05$ \\
\hline 7320 [O II] & $\ldots$ & $1.41 \pm 0.11$ & $1.07 \pm 0.04$ & $1.21 \pm 0.05$ \\
\hline 7330 [O II] & $\ldots$ & $1.00 \pm 0.10$ & $0.98 \pm 0.04$ & $0.93 \pm 0.04$ \\
\hline$C(\mathrm{H} \beta)$ & 0.350 & 0.235 & 0.145 & 0.590 \\
\hline
\end{tabular}


Table 4. continued.

\begin{tabular}{|c|c|c|c|c|}
\hline \multirow[b]{2}{*}{ Line } & \multicolumn{4}{|c|}{$\begin{array}{c}\text { GaLAXY } \\
\text { (ESO PROGRAM) }\end{array}$} \\
\hline & $\begin{array}{r}\text { UM 420 No.2 } \\
(69 . \mathrm{C}-0203(\mathrm{~A}))\end{array}$ & $\begin{array}{r}\text { CAM } 0357-3915 \\
(69 . \mathrm{C}-0203(\mathrm{~A}))\end{array}$ & $\begin{array}{r}\text { TOL 0513-393 } \\
(69 . \mathrm{C}-0203(\mathrm{~A}))\end{array}$ & $\begin{array}{r}\text { NGC 6822V No.1 } \\
(69 . \mathrm{C}-0203(\mathrm{~A}))\end{array}$ \\
\hline$F(\mathrm{H} \beta)^{a}$ & 13.99 & 74.42 & 68.84 & 94.56 \\
\hline$E W(\mathrm{H} \beta) \AA$ & 106.7 & 167.6 & 384.8 & 179.9 \\
\hline$E W(\mathrm{abs}) \AA$ & 0.40 & 0.65 & 5.95 & 0.50 \\
\hline \multirow[b]{2}{*}{ Line } & \multicolumn{4}{|c|}{$\begin{array}{c}\text { GaLAXY } \\
\text { (ESO PROGRAM) }\end{array}$} \\
\hline & $\begin{array}{r}\text { NGC 6822V No.2 } \\
(69 . C-0203(A))\end{array}$ & $\begin{array}{r}\text { TOL } 2138-405 \text { No.1 } \\
(69 . \mathrm{C}-0203(\mathrm{~A}))\end{array}$ & $\begin{array}{r}\text { TOL } 2146-391 \text { No.1 } \\
(69 . \mathrm{C}-0203(\mathrm{~A}))\end{array}$ & $\begin{array}{r}\text { TOL } 2146-391 \text { No.2 } \\
(69 . \mathrm{C}-0203(\mathrm{~A}))\end{array}$ \\
\hline 3727 [O II] & $88.08 \pm 2.44$ & $131.22 \pm 2.31$ & $80.98 \pm 1.59$ & $78.49 \pm 1.70$ \\
\hline $3750 \mathrm{H} 12$ & $5.92 \pm 1.34$ & $3.77 \pm 0.49$ & $4.83 \pm 0.59$ & $3.52 \pm 0.79$ \\
\hline $3771 \mathrm{H} 11$ & $4.61 \pm 1.20$ & $4.58 \pm 0.50$ & $5.19 \pm 0.59$ & $5.16 \pm 0.72$ \\
\hline 3798 H10 & $4.95 \pm 1.18$ & $6.07 \pm 0.48$ & $7.22 \pm 0.58$ & $6.47 \pm 0.65$ \\
\hline 3835 H9 & $7.32 \pm 1.06$ & $7.75 \pm 0.52$ & $9.43 \pm 0.59$ & $9.89 \pm 0.68$ \\
\hline 3868 [Ne III] & $43.70 \pm 1.03$ & $56.58 \pm 1.05$ & $48.06 \pm 0.93$ & $48.11 \pm 1.01$ \\
\hline $3889 \mathrm{He}_{\mathrm{I}}+\mathrm{H} 8$ & $19.66 \pm 1.06$ & $20.59 \pm 0.60$ & $20.58 \pm 0.65$ & $20.33 \pm 0.71$ \\
\hline $3968[\mathrm{Ne}$ III] + H7 & $30.60 \pm 1.12$ & $33.34 \pm 0.71$ & $31.40 \pm 0.75$ & $30.75 \pm 0.80$ \\
\hline $4026 \mathrm{He}_{\mathrm{I}}$ & $\ldots$ & $2.16 \pm 0.23$ & $2.01 \pm 0.21$ & $1.90 \pm 0.30$ \\
\hline $4068[\mathrm{~S} \mathrm{II}]$ & $\ldots$ & $\ldots$ & $1.63 \pm 0.22$ & $\ldots$ \\
\hline $4101 \mathrm{H} \delta$ & $26.75 \pm 0.95$ & $28.07 \pm 0.61$ & $27.23 \pm 0.65$ & $27.61 \pm 0.69$ \\
\hline $4340 \mathrm{H} \gamma$ & $47.79 \pm 1.05$ & $47.36 \pm 0.82$ & $48.87 \pm 0.88$ & $48.74 \pm 0.91$ \\
\hline 4363 [O III] & $6.63 \pm 0.27$ & $9.66 \pm 0.26$ & $13.27 \pm 0.30$ & $13.66 \pm 0.36$ \\
\hline $4387 \mathrm{He}_{\mathrm{I}}$ & $0.80 \pm 0.01$ & $\ldots$ & $\ldots$ & $\ldots$ \\
\hline $4471 \mathrm{He} \mathrm{I}$ & $3.87 \pm 0.19$ & $4.21 \pm 0.19$ & $3.58 \pm 0.15$ & $4.10 \pm 0.22$ \\
\hline 4658 [Fe III] & $\ldots$ & $1.04 \pm 0.13$ & $1.05 \pm 0.12$ & $0.57 \pm 0.10$ \\
\hline 4686 Не II & $\ldots$ & $1.63 \pm 0.14$ & $2.40 \pm 0.14$ & $2.07 \pm 0.18$ \\
\hline $4711[\mathrm{Ar}$ IV $]+\mathrm{He}_{\mathrm{I}}$ & $1.04 \pm 0.13$ & $1.48 \pm 0.13$ & $2.40 \pm 0.14$ & $2.43 \pm 0.17$ \\
\hline $4740[$ Ar Iv $]$ & $0.72 \pm 0.12$ & $1.06 \pm 0.14$ & $1.45 \pm 0.12$ & $1.14 \pm 0.13$ \\
\hline $4861 \mathrm{H} \beta$ & $100.00 \pm 1.63$ & $100.00 \pm 1.55$ & $100.00 \pm 1.56$ & $100.00 \pm 1.59$ \\
\hline $4921 \mathrm{He}_{\mathrm{I}}$ & $1.13 \pm 0.11$ & $0.83 \pm 0.12$ & $0.91 \pm 0.09$ & $0.91 \pm 0.12$ \\
\hline 4959 [O III] & $192.81 \pm 2.93$ & $226.88 \pm 3.41$ & $201.40 \pm 3.04$ & $194.04 \pm 2.98$ \\
\hline 4988 [Fe III] & $\ldots$ & $1.11 \pm 0.14$ & $0.95 \pm 0.09$ & $1.64 \pm 0.14$ \\
\hline $5007[\mathrm{O} \mathrm{III}]$ & $596.13 \pm 8.95$ & $687.30 \pm 10.25$ & $594.32 \pm 8.87$ & $573.40 \pm 8.68$ \\
\hline $5876 \mathrm{He}_{\mathrm{I}}$ & $11.49 \pm 0.23$ & $11.62 \pm 0.24$ & $9.92 \pm 0.20$ & $9.57 \pm 0.21$ \\
\hline $6300[\mathrm{O} \mathrm{I}]$ & $\ldots$ & $3.07 \pm 0.11$ & $2.15 \pm 0.08$ & $1.96 \pm 0.10$ \\
\hline 6312 [S III] & $1.78 \pm 0.08$ & $1.67 \pm 0.10$ & $1.56 \pm 0.07$ & $1.43 \pm 0.09$ \\
\hline $6363\left[\mathrm{O}_{\mathrm{I}}\right]$ & $\ldots$ & $0.96 \pm 0.08$ & $0.90 \pm 0.07$ & $0.71 \pm 0.08$ \\
\hline $6548[\mathrm{~N}$ II] & $1.01 \pm 0.07$ & $3.43 \pm 0.12$ & $0.98 \pm 0.06$ & $\ldots$ \\
\hline $6563 \mathrm{H} \alpha$ & $286.73 \pm 4.68$ & $283.59 \pm 4.61$ & $279.64 \pm 4.54$ & $278.97 \pm 4.60$ \\
\hline 6583 [N II] & $3.18 \pm 0.11$ & $12.24 \pm 0.25$ & $2.83 \pm 0.10$ & $2.39 \pm 0.11$ \\
\hline $6678 \mathrm{He}$ I & $2.99 \pm 0.10$ & $3.11 \pm 0.12$ & $2.78 \pm 0.20$ & $2.89 \pm 0.20$ \\
\hline 6717 [S II] & $5.01 \pm 0.12$ & $11.04 \pm 0.23$ & $6.65 \pm 0.15$ & $6.12 \pm 0.16$ \\
\hline 6731 [S II] & $3.67 \pm 0.10$ & $9.77 \pm 0.21$ & $5.32 \pm 0.13$ & $4.67 \pm 0.14$ \\
\hline $7065 \mathrm{He} \mathrm{I}$ & $2.51 \pm 0.09$ & $3.70 \pm 0.14$ & $2.89 \pm 0.09$ & $2.53 \pm 0.10$ \\
\hline 7136 [Ar III] & $8.87 \pm 0.18$ & $7.82 \pm 0.20$ & $4.67 \pm 0.12$ & $4.39 \pm 0.14$ \\
\hline $7281 \mathrm{He}_{\mathrm{I}}$ & $0.67 \pm 0.06$ & $\ldots$ & $\ldots$ & $\ldots$ \\
\hline 7320 [O II] & $1.18 \pm 0.06$ & $2.46 \pm 0.11$ & $1.24 \pm 0.07$ & $\ldots$ \\
\hline $7330[\mathrm{O}$ II] & $0.87 \pm 0.06$ & $1.96 \pm 0.12$ & $1.22 \pm 0.08$ & $\ldots$ \\
\hline$C(\mathrm{H} \beta)$ & 0.605 & 0.305 & 0.315 & 0.305 \\
\hline$F(\mathrm{H} \beta)^{a}$ & 38.46 & 34.51 & 66.98 & 50.64 \\
\hline$E W(\mathrm{H} \beta) \AA$ & 176.1 & 254.5 & 313.9 & 228.7 \\
\hline$E W($ abs $) \AA$ & 0.55 & 1.05 & 1.25 & 0.80 \\
\hline \multirow{3}{*}{ Line } & \multicolumn{2}{|c|}{$\begin{array}{c}\text { GaLAXY } \\
\text { (ESO ProGRam) }\end{array}$} & & \\
\hline & TOL 2240-384 & UM 160 No.1 & & \\
\hline & $(69 . \mathrm{C}-0203(\mathrm{~A}))$ & (69.C-0203(A)) & & \\
\hline 3727 [O II] & $64.03 \pm 1.31$ & $105.84 \pm 3.01$ & & \\
\hline $3750 \mathrm{H} 12$ & $4.59 \pm 0.83$ & $\ldots$ & & \\
\hline $3771 \mathrm{H} 11$ & $5.57 \pm 0.62$ & $\ldots$ & & \\
\hline 3798 H10 & $5.99 \pm 0.59$ & $\ldots$ & & \\
\hline $3820 \mathrm{He}_{\mathrm{I}}$ & $1.63 \pm 0.29$ & $\ldots$ & & \\
\hline 3835 H9 & $7.91 \pm 0.57$ & $7.11 \pm 1.14$ & & \\
\hline 3868 [Ne III] & $43.18 \pm 0.87$ & $32.67 \pm 1.07$ & & \\
\hline $3889 \mathrm{He}_{\mathrm{I}}+\mathrm{H} 8$ & $15.53 \pm 0.61$ & $20.91 \pm 1.29$ & & \\
\hline
\end{tabular}


Table 4. continued.

\begin{tabular}{|c|c|c|}
\hline \multirow[b]{2}{*}{ Line } & \multicolumn{2}{|c|}{$\begin{array}{c}\text { GALAXY } \\
\text { (ESO PROGRAM) }\end{array}$} \\
\hline & $\begin{array}{l}\text { TOL 2240-384 } \\
(69 . \mathrm{C}-0203(\mathrm{~A}))\end{array}$ & $\begin{array}{r}\text { UM } 160 \text { No.1 } \\
(69 . \mathrm{C}-0203(\mathrm{~A}))\end{array}$ \\
\hline $3968[\mathrm{Ne}$ III] + H7 & $29.49 \pm 0.71$ & $24.82 \pm 1.15$ \\
\hline $4026 \mathrm{He}$ I & $1.85 \pm 0.24$ & $\ldots$ \\
\hline $4068\left[\mathrm{~S}_{\mathrm{II}}\right]$ & $0.76 \pm 0.16$ & $\ldots$ \\
\hline $4101 \mathrm{H} \delta$ & $23.34 \pm 0.61$ & $23.30 \pm 1.06$ \\
\hline $4340 \mathrm{H} \gamma$ & $42.98 \pm 0.81$ & $44.20 \pm 1.16$ \\
\hline $4363[\mathrm{O} \mathrm{III]}$ & $13.87 \pm 0.32$ & $8.62 \pm 0.40$ \\
\hline $4471 \mathrm{He}_{\mathrm{I}}$ & $4.03 \pm 0.17$ & $4.04 \pm 0.27$ \\
\hline $4686 \mathrm{He}_{\text {II }}$ & $1.76 \pm 0.15$ & $\ldots$ \\
\hline $4711[\mathrm{Ar} \mathrm{IV}]+\mathrm{He}_{\mathrm{I}}$ & $2.49 \pm 0.15$ & $\ldots$ \\
\hline $4740[\mathrm{Ar}$ IV $]$ & $1.52 \pm 0.15$ & \\
\hline $4861 \mathrm{H} \beta$ & $100.00 \pm 1.60$ & $100.00 \pm 1.82$ \\
\hline $4921 \mathrm{He}$ I & $1.06 \pm 0.10$ & $\ldots$ \\
\hline 4959 [O III] & $213.39 \pm 3.29$ & $180.63 \pm 3.01$ \\
\hline 5007 [O III] & $644.11 \pm 9.83$ & $565.00 \pm 9.17$ \\
\hline 5755 [N II] & $0.48 \pm 0.08$ & $\ldots$ \\
\hline $5876 \mathrm{He}_{\mathrm{I}}$ & $12.99 \pm 0.25$ & $11.60 \pm 0.30$ \\
\hline $6300\left[\mathrm{O}_{\mathrm{I}}\right]$ & $2.13 \pm 0.08$ & $2.96 \pm 0.14$ \\
\hline 6312 [S III] & $0.87 \pm 0.07$ & $1.75 \pm 0.11$ \\
\hline $6363\left[\mathrm{O}_{\mathrm{I}}\right]$ & $0.72 \pm 0.07$ & $0.72 \pm 0.11$ \\
\hline $6563 \mathrm{H} \alpha$ & $282.54 \pm 4.69$ & $283.67 \pm 5.02$ \\
\hline $6583[\mathrm{~N} \mathrm{II]}$ & $5.01 \pm 0.14$ & $4.34 \pm 0.17$ \\
\hline $6678 \mathrm{He}_{\mathrm{I}}$ & $2.51 \pm 0.09$ & $3.02 \pm 0.15$ \\
\hline 6717 [S II] & $3.75 \pm 0.10$ & $10.21 \pm 0.26$ \\
\hline $6731\left[\mathrm{~S}_{\mathrm{II}}\right]$ & $3.05 \pm 0.09$ & $7.62 \pm 0.21$ \\
\hline $7065 \mathrm{He}_{\mathrm{I}}$ & $4.60 \pm 0.12$ & $3.26 \pm 0.14$ \\
\hline 7136 [Ar III] & $2.41 \pm 0.09$ & $5.77 \pm 0.19$ \\
\hline $7281 \mathrm{He}$ I & $\ldots$ & $0.64 \pm 0.09$ \\
\hline $7320[\mathrm{O} \mathrm{II}]$ & $\ldots$ & $1.85 \pm 0.11$ \\
\hline 7330 [O II] & $\ldots$ & $1.38 \pm 0.10$ \\
\hline$C(\mathrm{H} \beta)$ & 0.815 & 0.470 \\
\hline$F(\mathrm{H} \beta)^{a}$ & 72.83 & 27.43 \\
\hline$E W(\mathrm{H} \beta) \AA$ & 274.6 & 255.3 \\
\hline$E W($ abs $) \AA$ & 5.55 & 5.45 \\
\hline
\end{tabular}

Notes. ${ }^{(a)}$ In units $10^{-16} \mathrm{erg} \mathrm{s}^{-1} \mathrm{~cm}^{-2}$.

Table 5. Extinction-corrected emission line fluxes (medium-resolution FORS observations).

\begin{tabular}{|c|c|c|c|c|}
\hline \multirow[b]{2}{*}{ Line } & \multicolumn{4}{|c|}{$\begin{array}{c}\text { GALAXY } \\
\text { (ESO PROGRAM) }\end{array}$} \\
\hline & $\begin{array}{r}\text { NGC 346A No.1 } \\
(69 . \mathrm{C}-0203(\mathrm{~A}))\end{array}$ & $\begin{array}{r}\text { NGC 346A No.2 } \\
(69 . C-0203(A))\end{array}$ & $\begin{array}{r}\text { NGC 346A No.3 } \\
(69 . C-0203(A))\end{array}$ & $\begin{array}{r}\text { NGC 346B No.1 } \\
(69 . \mathrm{C}-0203(\mathrm{~A}))\end{array}$ \\
\hline 3727 [O II] & $89.43 \pm 1.39$ & $94.81 \pm 1.47$ & $116.32 \pm 1.81$ & $155.64 \pm 2.41$ \\
\hline $3750 \mathrm{H} 12$ & $4.54 \pm 0.13$ & $3.54 \pm 0.11$ & $2.95 \pm 0.23$ & $4.53 \pm 0.14$ \\
\hline $3771 \mathrm{H} 11$ & $5.24 \pm 0.13$ & $4.38 \pm 0.12$ & $4.00 \pm 0.21$ & $5.16 \pm 0.14$ \\
\hline 3798 H10 & $6.96 \pm 0.15$ & $5.62 \pm 0.13$ & $4.83 \pm 0.20$ & $6.47 \pm 0.15$ \\
\hline $3820 \mathrm{He}_{\mathrm{I}}$ & $1.15 \pm 0.05$ & $1.01 \pm 0.05$ & $\ldots$ & $1.30 \pm 0.07$ \\
\hline 3835 H9 & $8.93 \pm 0.17$ & $8.00 \pm 0.15$ & $6.91 \pm 0.21$ & $9.06 \pm 0.18$ \\
\hline 3868 [Ne III] & $45.71 \pm 0.70$ & $40.84 \pm 0.62$ & $42.26 \pm 0.66$ & $41.06 \pm 0.63$ \\
\hline $3889 \mathrm{He}_{\mathrm{I}}+\mathrm{H} 8$ & $22.98 \pm 0.36$ & $20.91 \pm 0.33$ & $20.24 \pm 0.34$ & $22.78 \pm 0.36$ \\
\hline $3968[\mathrm{Ne}$ III] + H7 & $31.27 \pm 0.48$ & $29.54 \pm 0.45$ & $27.14 \pm 0.44$ & $29.27 \pm 0.45$ \\
\hline 4026 He I & $2.12 \pm 0.05$ & $1.93 \pm 0.05$ & $\ldots$ & $2.39 \pm 0.06$ \\
\hline $4101 \mathrm{H} \delta$ & $28.73 \pm 0.43$ & $28.48 \pm 0.43$ & $26.31 \pm 0.41$ & $28.29 \pm 0.43$ \\
\hline $4340 \mathrm{H} \gamma$ & $50.18 \pm 0.73$ & $46.10 \pm 0.67$ & $47.19 \pm 0.69$ & $49.08 \pm 0.72$ \\
\hline 4363 [O III] & $7.43 \pm 0.11$ & $6.49 \pm 0.10$ & $6.28 \pm 0.12$ & $6.16 \pm 0.10$ \\
\hline $4387 \mathrm{He} \mathrm{I}$ & $0.54 \pm 0.02$ & $0.42 \pm 0.02$ & $\ldots$ & $\ldots$ \\
\hline $4471 \mathrm{He}_{\mathrm{I}}$ & $4.13 \pm 0.07$ & $4.06 \pm 0.07$ & $3.17 \pm 0.08$ & $4.12 \pm 0.07$ \\
\hline $4711[\mathrm{Ar} \mathrm{IV}]+\mathrm{He}_{\mathrm{I}}$ & $0.79 \pm 0.03$ & $1.31 \pm 0.04$ & $1.21 \pm 0.06$ & $\ldots$ \\
\hline 4740 [Ar Iv] & $0.21 \pm 0.02$ & $0.62 \pm 0.03$ & $0.48 \pm 0.05$ & $\ldots$ \\
\hline $4861 \mathrm{H} \beta$ & $100.00 \pm 1.43$ & $100.00 \pm 1.42$ & $100.00 \pm 1.43$ & $100.00 \pm 1.43$ \\
\hline $4921 \mathrm{He}_{\mathrm{I}}$ & $1.21 \pm 0.03$ & $1.00 \pm 0.01$ & $0.79 \pm 0.05$ & $1.10 \pm 0.03$ \\
\hline 4959 [O III] & $181.31 \pm 2.59$ & $173.49 \pm 2.47$ & $169.97 \pm 2.43$ & $163.68 \pm 2.34$ \\
\hline
\end{tabular}


Table 5. continued.

\begin{tabular}{|c|c|c|c|c|}
\hline \multirow[b]{2}{*}{ Line } & \multicolumn{4}{|c|}{$\begin{array}{c}\text { GALAXY } \\
\text { (ESO PROGRAM) }\end{array}$} \\
\hline & $\begin{array}{r}\text { NGC 346A No.1 } \\
(69 . C-0203(A))\end{array}$ & $\begin{array}{r}\text { NGC 346A No.2 } \\
(69 . C-0203(\mathrm{~A}))\end{array}$ & $\begin{array}{r}\text { NGC 346A No.3 } \\
(69 . \mathrm{C}-0203(\mathrm{~A}))\end{array}$ & $\begin{array}{r}\text { NGC 346B No.1 } \\
(69 . C-0203(A))\end{array}$ \\
\hline $4988[\mathrm{Fe} \mathrm{III]}$ & & & & $0.61 \pm 0.03$ \\
\hline 5007 [O III] & $547.45 \pm 7.81$ & $515.86 \pm 7.33$ & $483.33 \pm 6.90$ & $486.69 \pm 6.94$ \\
\hline $5015 \mathrm{He}_{\mathrm{I}}$ & $2.37 \pm 0.04$ & $3.19 \pm 0.05$ & $2.09 \pm 0.06$ & $2.48 \pm 0.04$ \\
\hline 5518 [Cl III] & $0.59 \pm 0.02$ & $0.32 \pm 0.02$ & $0.34 \pm 0.04$ & $0.36 \pm 0.02$ \\
\hline 5538 [Cl III] & $0.29 \pm 0.02$ & $0.27 \pm 0.02$ & $0.36 \pm 0.03$ & $0.25 \pm 0.02$ \\
\hline $5876 \mathrm{He} \mathrm{I}$ & $11.48 \pm 0.17$ & $10.76 \pm 0.16$ & $10.74 \pm 0.16$ & $11.10 \pm 0.17$ \\
\hline 6312 [S III] & $1.86 \pm 0.03$ & $1.29 \pm 0.03$ & $1.66 \pm 0.04$ & $1.91 \pm 0.04$ \\
\hline $6548[\mathrm{~N} \mathrm{II]}$ & $1.04 \pm 0.02$ & $1.54 \pm 0.03$ & $1.61 \pm 0.04$ & $2.02 \pm 0.04$ \\
\hline $6563 \mathrm{H} \alpha$ & $283.20 \pm 4.38$ & $283.39 \pm 4.37$ & $283.34 \pm 4.39$ & $283.81 \pm 4.39$ \\
\hline 6583 [N II] & $2.47 \pm 0.04$ & $3.93 \pm 0.07$ & $5.06 \pm 0.09$ & $5.35 \pm 0.09$ \\
\hline $6678 \mathrm{He}_{\mathrm{I}}$ & $3.08 \pm 0.05$ & $3.00 \pm 0.05$ & $2.96 \pm 0.06$ & $3.10 \pm 0.05$ \\
\hline 6717 [S II] & $4.05 \pm 0.07$ & $7.17 \pm 0.11$ & $7.78 \pm 0.13$ & $9.05 \pm 0.15$ \\
\hline $6731[\mathrm{~S} \mathrm{II]}$ & $2.83 \pm 0.05$ & $5.19 \pm 0.08$ & $5.67 \pm 0.10$ & $6.65 \pm 0.11$ \\
\hline $7065 \mathrm{He}$ I & $2.04 \pm 0.04$ & $2.07 \pm 0.04$ & $2.07 \pm 0.04$ & $2.16 \pm 0.04$ \\
\hline 7136 [Ar III] & $8.00 \pm 0.13$ & $6.88 \pm 0.11$ & $7.95 \pm 0.13$ & $8.12 \pm 0.13$ \\
\hline$C(\mathrm{H} \beta)$ & 0.165 & 0.070 & 0.090 & 0.165 \\
\hline$F(\mathrm{H} \beta)^{a}$ & 517.00 & 381.30 & 301.10 & 456.30 \\
\hline$E W(\mathrm{H} \beta) \AA$ & 286.1 & 309.2 & 67.5 & 241.0 \\
\hline \multirow[t]{2}{*}{$E W($ abs $) \AA$} & 0.90 & 0.35 & 0.25 & 0.80 \\
\hline & \multicolumn{4}{|c|}{ Galaxy } \\
\hline Line & NGC 346B No.2 & NGC 346D No.1 & NGC 346D No.2 & NGC 346D No.3 \\
\hline & (69.C-0203(A)) & (69.C-0203(A)) & (69.C-0203(A)) & (69.C-0203(A)) \\
\hline 3727 [O II] & $84.73 \pm 1.31$ & $177.00 \pm 2.72$ & $87.73 \pm 1.36$ & $115.25 \pm 1.78$ \\
\hline 3750 H12 & $3.34 \pm 0.13$ & $3.73 \pm 0.10$ & $3.72 \pm 0.11$ & $3.77 \pm 0.10$ \\
\hline $3771 \mathrm{H} 11$ & $4.26 \pm 0.13$ & $5.05 \pm 0.10$ & $4.57 \pm 0.11$ & $4.83 \pm 0.10$ \\
\hline 3798 H10 & $5.38 \pm 0.13$ & $5.72 \pm 0.11$ & $5.63 \pm 0.12$ & $5.92 \pm 0.12$ \\
\hline $3820 \mathrm{He}_{\mathrm{I}}$ & $\ldots$ & $1.12 \pm 0.05$ & $0.96 \pm 0.05$ & $0.98 \pm 0.04$ \\
\hline 3835 H9 & $7.71 \pm 0.16$ & $8.00 \pm 0.14$ & $7.81 \pm 0.15$ & $8.16 \pm 0.14$ \\
\hline 3868 [Ne III] & $38.78 \pm 0.59$ & $38.71 \pm 0.59$ & $39.64 \pm 0.61$ & $40.21 \pm 0.61$ \\
\hline $3889 \mathrm{He}_{\mathrm{I}}+\mathrm{H} 8$ & $20.93 \pm 0.33$ & $21.39 \pm 0.33$ & $21.18 \pm 0.33$ & $21.85 \pm 0.34$ \\
\hline 3968 [Ne III] + H7 & $28.33 \pm 0.44$ & $28.90 \pm 0.44$ & $29.01 \pm 0.44$ & $29.82 \pm 0.45$ \\
\hline $4026 \mathrm{He}_{\mathrm{I}}$ & $1.88 \pm 0.06$ & $1.96 \pm 0.04$ & $1.97 \pm 0.05$ & $2.09 \pm 0.05$ \\
\hline 4068 [S II] & $\ldots$ & $1.24 \pm 0.03$ & $0.78 \pm 0.03$ & $0.97 \pm 0.03$ \\
\hline 4076 [S II] & $\ldots$ & $0.40 \pm 0.02$ & $0.26 \pm 0.03$ & $0.38 \pm 0.02$ \\
\hline $4101 \mathrm{H} \delta$ & $27.31 \pm 0.41$ & $28.07 \pm 0.42$ & $26.93 \pm 0.41$ & $27.77 \pm 0.42$ \\
\hline $4340 \mathrm{H} \gamma$ & $46.63 \pm 0.68$ & $48.43 \pm 0.70$ & $46.65 \pm 0.68$ & $49.83 \pm 0.73$ \\
\hline 4363 [O III] & $6.34 \pm 0.10$ & $6.37 \pm 0.10$ & $6.46 \pm 0.10$ & $6.43 \pm 0.10$ \\
\hline $4387 \mathrm{He}$ I & 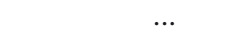 & $0.60 \pm 0.03$ & $0.54 \pm 0.03$ & $0.68 \pm 0.03$ \\
\hline $4471 \mathrm{He}_{\mathrm{I}}$ & $3.87 \pm 0.07$ & $3.90 \pm 0.06$ & $4.08 \pm 0.07$ & $4.19 \pm 0.07$ \\
\hline $4711[\mathrm{Ar}$ IV $]+\mathrm{He}_{\mathrm{I}}$ & $\ldots$ & $0.68 \pm 0.02$ & $0.79 \pm 0.03$ & $\ldots$ \\
\hline 4740 [Ar Iv] & $\ldots$ & $0.16 \pm 0.01$ & $0.24 \pm 0.02$ & $\ldots$ \\
\hline $4861 \mathrm{H} \beta$ & $100.00 \pm 1.42$ & $100.00 \pm 1.42$ & $100.00 \pm 1.43$ & $100.00 \pm 1.43$ \\
\hline $4921 \mathrm{He}$ I & $1.12 \pm 0.03$ & $1.04 \pm 0.02$ & $1.09 \pm 0.03$ & $1.24 \pm 0.03$ \\
\hline 4959 [O III] & $170.90 \pm 2.43$ & $159.33 \pm 2.27$ & $170.72 \pm 2.43$ & $164.75 \pm 2.35$ \\
\hline $4988[\mathrm{Fe}$ III] & $0.89 \pm 0.03$ & $0.23 \pm 0.02$ & $0.30 \pm 0.02$ & \\
\hline 5007 [O III] & $503.17 \pm 7.15$ & $470.52 \pm 6.69$ & $518.55 \pm 7.38$ & $480.11 \pm 6.84$ \\
\hline $5015 \mathrm{He}_{\mathrm{I}}$ & $2.41 \pm 0.04$ & $2.54 \pm 0.04$ & $2.35 \pm 0.04$ & $2.72 \pm 0.05$ \\
\hline 5518 [Cl III] & $0.38 \pm 0.02$ & $0.39 \pm 0.01$ & $0.39 \pm 0.02$ & $0.39 \pm 0.02$ \\
\hline $5538[\mathrm{Cl} \mathrm{III}]$ & $0.23 \pm 0.02$ & $0.38 \pm 0.01$ & $0.39 \pm 0.02$ & $0.37 \pm 0.02$ \\
\hline $5876 \mathrm{He} \mathrm{I}$ & $10.78 \pm 0.16$ & $10.19 \pm 0.15$ & $10.75 \pm 0.16$ & $11.04 \pm 0.17$ \\
\hline 6312 [S III] & $1.92 \pm 0.03$ & $1.86 \pm 0.03$ & $1.81 \pm 0.04$ & $2.06 \pm 0.04$ \\
\hline 6548 [N II] & $1.18 \pm 0.03$ & $3.23 \pm 0.05$ & $1.95 \pm 0.04$ & $2.26 \pm 0.04$ \\
\hline $6563 \mathrm{H} \alpha$ & $283.41 \pm 4.37$ & $283.02 \pm 4.37$ & $283.36 \pm 4.38$ & $282.95 \pm 4.37$ \\
\hline 6583 [N II] & $2.87 \pm 0.05$ & $6.72 \pm 0.11$ & $3.29 \pm 0.06$ & $4.71 \pm 0.08$ \\
\hline $6678 \mathrm{He}$ I & $3.26 \pm 0.05$ & $2.85 \pm 0.05$ & $2.95 \pm 0.05$ & $3.04 \pm 0.05$ \\
\hline 6717 [S II] & $4.23 \pm 0.07$ & $12.00 \pm 0.19$ & $6.16 \pm 0.10$ & $7.71 \pm 0.12$ \\
\hline 6731 [S II] & $3.03 \pm 0.05$ & $8.51 \pm 0.14$ & $4.36 \pm 0.07$ & $5.98 \pm 0.10$ \\
\hline $7065 \mathrm{He}_{\mathrm{I}}$ & $2.04 \pm 0.04$ & $1.99 \pm 0.04$ & $2.09 \pm 0.04$ & $2.19 \pm 0.04$ \\
\hline 7136 [Ar III] & $7.24 \pm 0.12$ & $7.67 \pm 0.13$ & $7.68 \pm 0.13$ & $7.99 \pm 0.13$ \\
\hline$C(\mathrm{H} \beta)$ & 0.070 & 0.115 & 0.060 & 0.070 \\
\hline$F(\mathrm{H} \beta)^{a}$ & 557.60 & 536.00 & 359.30 & 392.60 \\
\hline
\end{tabular}


Table 5. continued.

\begin{tabular}{|c|c|c|c|c|}
\hline \multirow[b]{2}{*}{ Line } & \multicolumn{4}{|c|}{$\begin{array}{c}\text { GALAXY } \\
\text { (ESO PROGRAM) }\end{array}$} \\
\hline & $\begin{array}{r}\text { NGC 346B No.2 } \\
(69 . \mathrm{C}-0203(\mathrm{~A}))\end{array}$ & $\begin{array}{r}\text { NGC 346D No.1 } \\
(69 . \mathrm{C}-0203(\mathrm{~A}))\end{array}$ & $\begin{array}{r}\text { NGC 346D No.2 } \\
(69 . \mathrm{C}-0203(\mathrm{~A}))\end{array}$ & $\begin{array}{r}\text { NGC 346D No.3 } \\
(69 . \mathrm{C}-0203(\mathrm{~A}))\end{array}$ \\
\hline$E W(\mathrm{H} \beta) \AA$ & 178.7 & 349.2 & 367.1 & 378.9 \\
\hline$E W(\mathrm{abs}) \AA$ & 0.25 & 0.45 & 1.00 & 1.35 \\
\hline \multirow[b]{2}{*}{ Line } & \multicolumn{4}{|c|}{$\begin{array}{c}\text { GALAXY } \\
\text { (ESO PROGRAM) }\end{array}$} \\
\hline & $\begin{array}{r}\text { NGC 346D No.4 } \\
(69 . \mathrm{C}-0203(\mathrm{~A}))\end{array}$ & $\begin{array}{r}\text { NGC 456A No.1 } \\
(69 . \mathrm{C}-0203(\mathrm{~A}))\end{array}$ & $\begin{array}{r}\text { NGC 456A No.2 } \\
(69 . \mathrm{C}-0203(\mathrm{~A}))\end{array}$ & $\begin{array}{r}\text { NGC 456A No.3 } \\
(69 . C-0203(A))\end{array}$ \\
\hline $3727[\mathrm{O} \mathrm{II]}$ & $123.48 \pm 1.91$ & $229.94 \pm 3.55$ & $132.81 \pm 2.08$ & $170.10 \pm 2.64$ \\
\hline $3750 \mathrm{H} 12$ & $3.59 \pm 0.12$ & $3.68 \pm 0.09$ & $6.42 \pm 0.23$ & $4.43 \pm 0.12$ \\
\hline 3771 H11 & $4.56 \pm 0.12$ & $4.69 \pm 0.10$ & $7.15 \pm 0.20$ & $10.75 \pm 0.19$ \\
\hline 3798 H10 & $5.65 \pm 0.13$ & $5.99 \pm 0.11$ & $9.13 \pm 0.20$ & $6.47 \pm 0.13$ \\
\hline $3820 \mathrm{He}_{\mathrm{I}}$ & $1.10 \pm 0.05$ & $1.20 \pm 0.04$ & $\ldots$ & $1.00 \pm 0.04$ \\
\hline 3835 H9 & $7.99 \pm 0.15$ & $8.79 \pm 0.15$ & $9.94 \pm 0.20$ & $8.90 \pm 0.16$ \\
\hline 3868 [Ne III] & $41.21 \pm 0.63$ & $24.69 \pm 0.38$ & $27.81 \pm 0.44$ & $25.33 \pm 0.40$ \\
\hline $3889 \mathrm{He}_{\mathrm{I}}+\mathrm{H} 8$ & $21.49 \pm 0.34$ & $20.71 \pm 0.32$ & $21.59 \pm 0.35$ & $21.73 \pm 0.34$ \\
\hline $3968[\mathrm{Ne}$ III] + H7 & $29.38 \pm 0.45$ & $25.22 \pm 0.38$ & $26.88 \pm 0.42$ & $25.64 \pm 0.40$ \\
\hline $4026 \mathrm{He}_{\mathrm{I}}$ & $2.05 \pm 0.05$ & $1.97 \pm 0.04$ & $1.51 \pm 0.05$ & $2.14 \pm 0.04$ \\
\hline $4068[\mathrm{~S}$ II $]$ & $0.94 \pm 0.04$ & $1.30 \pm 0.03$ & $0.73 \pm 0.04$ & $0.75 \pm 0.03$ \\
\hline $4076[$ S II] & $0.33 \pm 0.04$ & $0.48 \pm 0.02$ & $0.26 \pm 0.04$ & $0.30 \pm 0.03$ \\
\hline $4101 \mathrm{H} \delta$ & $28.03 \pm 0.42$ & $27.15 \pm 0.41$ & $27.52 \pm 0.42$ & $27.01 \pm 0.41$ \\
\hline $4340 \mathrm{H} \gamma$ & $47.16 \pm 0.69$ & $46.17 \pm 0.67$ & $45.76 \pm 0.68$ & $46.40 \pm 0.68$ \\
\hline 4363 [O III] & $6.82 \pm 0.11$ & $4.39 \pm 0.07$ & $4.75 \pm 0.09$ & $4.44 \pm 0.07$ \\
\hline $4387 \mathrm{He} \mathrm{I}$ & $0.48 \pm 0.03$ & $0.45 \pm 0.02$ & $0.34 \pm 0.03$ & $0.52 \pm 0.03$ \\
\hline $4471 \mathrm{He} \mathrm{I}$ & $4.00 \pm 0.07$ & $4.16 \pm 0.06$ & $3.58 \pm 0.07$ & $3.97 \pm 0.07$ \\
\hline 4658 [Fe III] & & $0.32 \pm 0.02$ & $0.29 \pm 0.03$ & $0.36 \pm 0.01$ \\
\hline $4711[\mathrm{Ar}$ Iv $]+\mathrm{He}_{\mathrm{I}}$ & $0.88 \pm 0.03$ & $0.54 \pm 0.01$ & $\ldots$ & $\ldots$ \\
\hline $4740[\mathrm{Ar}$ Iv $]$ & $0.32 \pm 0.02$ & $\ldots$ & $\ldots$ & $\ldots$ \\
\hline $4861 \mathrm{H} \beta$ & $100.00 \pm 1.43$ & $100.00 \pm 1.43$ & $100.00 \pm 1.44$ & $100.00 \pm 1.44$ \\
\hline $4921 \mathrm{He}_{\mathrm{I}}$ & $1.06 \pm 0.03$ & $1.08 \pm 0.02$ & $0.93 \pm 0.03$ & $1.13 \pm 0.03$ \\
\hline 4959 [O III] & $161.94 \pm 2.31$ & $124.26 \pm 1.77$ & $142.46 \pm 2.06$ & $133.81 \pm 1.92$ \\
\hline $4988[\mathrm{Fe} \mathrm{III}]$ & $0.49 \pm 0.03$ & $0.25 \pm 0.02$ & $0.29 \pm 0.02$ & $0.35 \pm 0.02$ \\
\hline 5007 [O III] & $498.36 \pm 7.10$ & $370.72 \pm 5.29$ & $425.09 \pm 6.13$ & $390.36 \pm 5.59$ \\
\hline $5015 \mathrm{He}_{\mathrm{I}}$ & $2.63 \pm 0.05$ & $1.97 \pm 0.03$ & $2.32 \pm 0.05$ & $1.93 \pm 0.04$ \\
\hline 5518 [Cl III] & $0.38 \pm 0.02$ & $0.35 \pm 0.01$ & $0.34 \pm 0.02$ & $0.35 \pm 0.02$ \\
\hline $5538[\mathrm{Cl} \mathrm{III}]$ & $0.37 \pm 0.02$ & $0.30 \pm 0.01$ & $0.28 \pm 0.02$ & $0.27 \pm 0.02$ \\
\hline $5755[\mathrm{~N} \mathrm{II}]$ & & $0.18 \pm 0.01$ & $0.09 \pm 0.02$ & $0.12 \pm 0.02$ \\
\hline $5876 \mathrm{He}_{\mathrm{I}}$ & $10.94 \pm 0.17$ & $11.16 \pm 0.17$ & $10.36 \pm 0.16$ & $10.98 \pm 0.17$ \\
\hline 6312 [S III] & $1.72 \pm 0.03$ & $1.83 \pm 0.03$ & $1.62 \pm 0.03$ & $1.83 \pm 0.03$ \\
\hline 6548 [N II] & $2.26 \pm 0.04$ & $2.75 \pm 0.05$ & $1.77 \pm 0.04$ & $1.79 \pm 0.03$ \\
\hline $6563 \mathrm{H} \alpha$ & $282.80 \pm 4.37$ & $284.40 \pm 4.40$ & $283.99 \pm 4.44$ & $284.58 \pm 4.42$ \\
\hline 6583 [N II] & $4.43 \pm 0.07$ & $8.02 \pm 0.13$ & $4.85 \pm 0.08$ & $5.19 \pm 0.09$ \\
\hline $6678 \mathrm{He}_{\mathrm{I}}$ & $3.01 \pm 0.05$ & $3.14 \pm 0.05$ & $3.09 \pm 0.06$ & $3.10 \pm 0.05$ \\
\hline 6717 [S II] & $8.12 \pm 0.13$ & $9.28 \pm 0.15$ & $7.46 \pm 0.12$ & $6.71 \pm 0.11$ \\
\hline $6731[\mathrm{~S}$ II] & $6.14 \pm 0.10$ & $8.15 \pm 0.13$ & $5.56 \pm 0.09$ & $5.12 \pm 0.08$ \\
\hline $7065 \mathrm{He}_{\mathrm{I}}$ & $2.35 \pm 0.04$ & $2.57 \pm 0.04$ & $2.32 \pm 0.05$ & $2.20 \pm 0.04$ \\
\hline 7136 [Ar III] & $7.80 \pm 0.13$ & $8.21 \pm 0.13$ & $8.36 \pm 0.14$ & $8.08 \pm 0.13$ \\
\hline $7320[\mathrm{O}$ II] & $\ldots$ & $4.00 \pm 0.07$ & $1.94 \pm 0.04$ & $2.23 \pm 0.04$ \\
\hline 7330 [O II] & $\ldots$ & $3.16 \pm 0.05$ & $1.62 \pm 0.03$ & $1.82 \pm 0.03$ \\
\hline$C(\mathrm{H} \beta)$ & 0.100 & 0.190 & 0.000 & 0.155 \\
\hline$F(\mathrm{H} \beta)^{a}$ & 405.80 & 377.60 & 231.70 & 255.50 \\
\hline$E W(\mathrm{H} \beta) \AA$ & 296.0 & 655.7 & 219.4 & 567.8 \\
\hline$E W(\mathrm{abs}) \AA$ & 0.70 & 1.50 & 3.20 & 3.65 \\
\hline \multirow[b]{2}{*}{ Line } & \multicolumn{4}{|c|}{$\begin{array}{c}\text { GALAXY } \\
\text { (ESO PROGRAM) }\end{array}$} \\
\hline & UM 420 No.1 & UM 420 No. 2 & CAM 0357-3915 & TOL 0513-393 \\
\hline $3727[\mathrm{O}$ II] & $330.35 \pm 5.69$ & $\frac{1.0-(200(A))}{280.57 \pm 5.00}$ & $\frac{9 .(-0205(A))}{88.25 \pm 1.43}$ & $\frac{9 .-020(A))}{55.29 \pm 0.89}$ \\
\hline $3750 \mathrm{H} 12$ & $\ldots$ & $2.94 \pm 0.68$ & $4.18 \pm 0.23$ & $4.36 \pm 0.16$ \\
\hline 3771 H11 & $\ldots$ & $4.73 \pm 0.71$ & $4.81 \pm 0.23$ & $5.21 \pm 0.16$ \\
\hline 3798 H10 & $4.44 \pm 0.72$ & $6.47 \pm 0.70$ & $6.49 \pm 0.25$ & $6.53 \pm 0.17$ \\
\hline $3820 \mathrm{He} \mathrm{I}$ & $\ldots$ & $\ldots$ & $0.62 \pm 0.11$ & $0.78 \pm 0.05$ \\
\hline 3835 H9 & $6.86 \pm 0.64$ & $8.16 \pm 0.78$ & $8.04 \pm 0.24$ & $8.13 \pm 0.17$ \\
\hline $3868[\mathrm{Ne}$ III] & $38.09 \pm 0.90$ & $52.96 \pm 1.18$ & $51.05 \pm 0.83$ & $53.55 \pm 0.84$ \\
\hline
\end{tabular}


Table 5. continued.

\begin{tabular}{|c|c|c|c|c|}
\hline \multirow{3}{*}{ Line } & \multicolumn{4}{|c|}{$\begin{array}{l}\text { GALAXY } \\
\text { SO PROGRAM) }\end{array}$} \\
\hline & UM 420 No. 1 & UM 420 No. 2 & CAM 0357-3915 & TOL 0513-393 \\
\hline & (69.C-0203(A)) & (69.C-0203(A)) & (69.C-0203(A)) & $(69 . \mathrm{C}-0203(\mathrm{~A}))$ \\
\hline $3889 \mathrm{He}_{\mathrm{I}}+\mathrm{H} 8$ & $20.93 \pm 0.79$ & $23.26 \pm 0.83$ & $20.59 \pm 0.39$ & $18.67 \pm 0.32$ \\
\hline $3968[\mathrm{Ne}$ III $]+\mathrm{H} 7$ & $24.76 \pm 0.81$ & $34.22 \pm 0.98$ & $31.83 \pm 0.55$ & $32.82 \pm 0.52$ \\
\hline $4026 \mathrm{He}_{\mathrm{I}}$ & $1.92 \pm 0.40$ & $2.12 \pm 0.39$ & $1.42 \pm 0.09$ & $1.62 \pm 0.06$ \\
\hline $4068[\mathrm{~S}$ II $]$ & $3.76 \pm 0.39$ & $\ldots$ & $0.79 \pm 0.08$ & $0.65 \pm 0.05$ \\
\hline 4076 [S II] & $\ldots$ & $\ldots$ & $0.46 \pm 0.08$ & $0.28 \pm 0.05$ \\
\hline $4101 \mathrm{H} \delta$ & $27.18 \pm 0.79$ & $31.84 \pm 0.85$ & $26.04 \pm 0.44$ & $25.23 \pm 0.40$ \\
\hline $4340 \mathrm{H} \gamma$ & $46.74 \pm 0.98$ & $54.37 \pm 1.08$ & $47.23 \pm 0.73$ & $44.52 \pm 0.67$ \\
\hline 4363 [O III] & $4.44 \pm 0.38$ & $7.77 \pm 0.38$ & $12.54 \pm 0.23$ & $14.84 \pm 0.24$ \\
\hline $4387 \mathrm{He}_{\mathrm{I}}$ & $\ldots$ & $\ldots$ & & $0.45 \pm 0.04$ \\
\hline $4471 \mathrm{He}_{\mathrm{I}}$ & $4.00 \pm 0.37$ & $4.26 \pm 0.30$ & $3.72 \pm 0.11$ & $3.81 \pm 0.08$ \\
\hline 4658 [Fe III] & $1.61 \pm 0.27$ & $1.65 \pm 0.24$ & $0.60 \pm 0.09$ & $0.45 \pm 0.04$ \\
\hline $4686 \mathrm{He}_{\text {II }}$ & $\ldots$ & $\ldots$ & $1.70 \pm 0.08$ & $0.86 \pm 0.04$ \\
\hline 4711 [Ar Iv] + He I & $\ldots$ & $\ldots$ & $1.73 \pm 0.09$ & $2.54 \pm 0.06$ \\
\hline $4740[\mathrm{Ar} \mathrm{Iv}]$ & $\ldots$ & $\ldots$ & $0.94 \pm 0.06$ & $1.63 \pm 0.05$ \\
\hline $4861 \mathrm{H} \beta$ & $100.00 \pm 1.68$ & $100.00 \pm 1.66$ & $100.00 \pm 1.48$ & $100.00 \pm 1.46$ \\
\hline $4921 \mathrm{He}_{\mathrm{I}}$ & $1.19 \pm 0.24$ & $0.92 \pm 0.25$ & $0.84 \pm 0.07$ & $1.05 \pm 0.04$ \\
\hline 4959 [O III] & $131.01 \pm 2.11$ & $171.32 \pm 2.74$ & $209.95 \pm 3.07$ & $254.82 \pm 3.70$ \\
\hline 4988 [Fe III] & $1.78 \pm 0.27$ & $1.32 \pm 0.21$ & $0.80 \pm 0.06$ & $0.46 \pm 0.04$ \\
\hline 5007 [O III] & $386.07 \pm 6.01$ & $495.37 \pm 7.74$ & $639.37 \pm 9.31$ & $748.73 \pm 10.84$ \\
\hline $5015 \mathrm{He}_{\mathrm{I}}$ & $\ldots$ & $\ldots$ & & $1.43 \pm 0.05$ \\
\hline $5518[\mathrm{Cl} \mathrm{III}]$ & $\ldots$ & $\ldots$ & $0.29 \pm 0.05$ & $0.40 \pm 0.03$ \\
\hline $5538[\mathrm{Cl} \mathrm{III}]$ & $\ldots$ & $\ldots$ & $0.22 \pm 0.05$ & $0.22 \pm 0.03$ \\
\hline $5876 \mathrm{He}_{\mathrm{I}}$ & $11.33 \pm 0.34$ & $11.04 \pm 0.27$ & $10.67 \pm 0.18$ & $12.41 \pm 0.20$ \\
\hline $6300\left[\mathrm{O}_{\mathrm{I}}\right]$ & $7.20 \pm 0.31$ & $5.54 \pm 0.18$ & $2.20 \pm 0.06$ & $2.18 \pm 0.05$ \\
\hline 6312 [S III] & $1.52 \pm 0.21$ & $1.64 \pm 0.12$ & $1.19 \pm 0.05$ & $1.48 \pm 0.04$ \\
\hline $6363\left[\mathrm{O}_{\mathrm{I}}\right]$ & $1.99 \pm 0.23$ & $1.88 \pm 0.12$ & $0.71 \pm 0.04$ & $0.69 \pm 0.03$ \\
\hline $6548[\mathrm{~N}$ II] & $6.91 \pm 0.30$ & $3.84 \pm 0.15$ & $1.43 \pm 0.05$ & \\
\hline $6563 \mathrm{H} \alpha$ & $281.18 \pm 4.78$ & $284.10 \pm 4.82$ & $280.44 \pm 4.43$ & $279.42 \pm 4.40$ \\
\hline 6583 [N II] & $23.97 \pm 0.55$ & $11.92 \pm 0.27$ & $4.95 \pm 0.10$ & $4.26 \pm 0.09$ \\
\hline $6678 \mathrm{He}_{\mathrm{I}}$ & $3.27 \pm 0.30$ & $2.85 \pm 0.13$ & $2.81 \pm 0.07$ & $3.47 \pm 0.08$ \\
\hline 6717 [S II] & $32.88 \pm 0.69$ & $19.51 \pm 0.40$ & $5.82 \pm 0.11$ & $6.24 \pm 0.12$ \\
\hline 6731 [S II] & $23.91 \pm 0.54$ & $14.34 \pm 0.32$ & $4.74 \pm 0.10$ & $5.24 \pm 0.10$ \\
\hline $7065 \mathrm{He}_{\mathrm{I}}$ & $2.54 \pm 0.20$ & $2.86 \pm 0.12$ & $2.95 \pm 0.18$ & $5.03 \pm 0.10$ \\
\hline$C(\mathrm{H} \beta)$ & 0.000 & 0.560 & 0.250 & 0.060 \\
\hline$F(\mathrm{H} \beta)^{a}$ & 12.06 & 12.06 & 46.92 & 76.95 \\
\hline$E W(\mathrm{H} \beta) \AA$ & 50.7 & 10.5 & 174.7 & 398.3 \\
\hline$E W($ abs $) \AA$ & 0.00 & 0.25 & 1.20 & 4.85 \\
\hline \multirow{3}{*}{ Line } & \multicolumn{4}{|c|}{ GaLaXY } \\
\hline & TOL 1214-277 & TOL 65 No.1 & TOL 65 No.2 & NGC 6822V No.1 \\
\hline & (65.N-0642(A)) & $5 . \mathrm{N}-0642(\mathrm{~A})$ & $(65 . \mathrm{N}-0642(\mathrm{~A}))$ & $(69 . \mathrm{C}-0203(\mathrm{~A}))$ \\
\hline $3727[\mathrm{O} \mathrm{II]}$ & $29.99 \pm 0.50$ & $69.99 \pm 1.11$ & $102.15 \pm 1.72$ & $77.03 \pm 1.27$ \\
\hline $3750 \mathrm{H} 12$ & $3.72 \pm 0.15$ & $4.54 \pm 0.21$ & $2.85 \pm 0.35$ & $2.98 \pm 0.25$ \\
\hline 3771 H11 & $4.23 \pm 0.15$ & $5.03 \pm 0.19$ & $3.34 \pm 0.37$ & $3.87 \pm 0.23$ \\
\hline 3798 H10 & $5.94 \pm 0.16$ & $6.62 \pm 0.19$ & $5.33 \pm 0.36$ & $4.93 \pm 0.23$ \\
\hline $3820 \mathrm{He} \mathrm{I}$ & $1.14 \pm 0.07$ & $1.04 \pm 0.08$ & $\ldots$ & $1.04 \pm 0.15$ \\
\hline 3835 H9 & $6.09 \pm 0.16$ & $8.44 \pm 0.20$ & $6.20 \pm 0.36$ & $6.99 \pm 0.25$ \\
\hline $3868[\mathrm{Ne}$ III] & $34.30 \pm 0.54$ & $32.18 \pm 0.51$ & $31.69 \pm 0.59$ & $43.74 \pm 0.72$ \\
\hline $3889 \mathrm{He}_{\mathrm{I}}+\mathrm{H} 8$ & $20.41 \pm 0.34$ & $21.70 \pm 0.37$ & $20.89 \pm 0.48$ & $19.03 \pm 0.38$ \\
\hline $3968[\mathrm{Ne}$ III $]+\mathrm{H} 7$ & $29.51 \pm 0.47$ & $26.88 \pm 0.43$ & $24.38 \pm 0.51$ & $28.85 \pm 0.51$ \\
\hline $4026 \mathrm{He}$ I & $1.67 \pm 0.07$ & $1.55 \pm 0.06$ & $1.12 \pm 0.16$ & $1.51 \pm 0.12$ \\
\hline 4068 [S II] & $0.41 \pm 0.05$ & $0.76 \pm 0.06$ & $1.43 \pm 0.19$ & $0.56 \pm 0.09$ \\
\hline 4076 [S II] & $\ldots$ & $\ldots$ & $\ldots$ & $0.28 \pm 0.09$ \\
\hline $4101 \mathrm{H} \delta$ & $27.00 \pm 0.42$ & $27.01 \pm 0.42$ & $26.82 \pm 0.51$ & $25.52 \pm 0.44$ \\
\hline $4227[\mathrm{Fe} \mathrm{v}]$ & $1.00 \pm 0.10$ & $\ldots$ & & \\
\hline $4340 \mathrm{H} \gamma$ & $48.47 \pm 0.72$ & $47.09 \pm 0.70$ & $48.72 \pm 0.78$ & $46.67 \pm 0.72$ \\
\hline 4363 [O III] & $17.24 \pm 0.26$ & $10.36 \pm 0.17$ & $9.13 \pm 0.23$ & $6.27 \pm 0.13$ \\
\hline $4387 \mathrm{He} \mathrm{I}$ & $0.38 \pm 0.05$ & $0.36 \pm 0.04$ & & $\ldots$ \\
\hline $4471 \mathrm{He}$ I & $3.56 \pm 0.07$ & $3.61 \pm 0.07$ & $3.08 \pm 0.14$ & $3.77 \pm 0.10$ \\
\hline 4658 [Fe III] & $0.33 \pm 0.03$ & $0.42 \pm 0.04$ & $0.68 \pm 0.10$ & $\ldots$ \\
\hline 4686 Не II & $5.34 \pm 0.10$ & $0.71 \pm 0.04$ & $2.00 \pm 0.13$ & 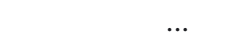 \\
\hline $4711[\mathrm{Ar} \mathrm{IV}]+\mathrm{He}$ & $2.91 \pm 0.06$ & $1.22 \pm 0.04$ & $0.64 \pm 0.13$ & $0.92 \pm 0.07$ \\
\hline
\end{tabular}


Table 5. continued.

\begin{tabular}{|c|c|c|c|c|}
\hline \multirow[b]{2}{*}{ Line } & \multicolumn{4}{|c|}{$\begin{array}{c}\text { GALAXY } \\
\text { (ESO PROGRAM) }\end{array}$} \\
\hline & $\begin{array}{l}\text { TOL 1214-277 } \\
(65 . \mathrm{N}-0642(\mathrm{~A}))\end{array}$ & $\begin{array}{r}\text { TOL } 65 \text { No. } 1 \\
(65 . \mathrm{N}-0642(\mathrm{~A}))\end{array}$ & $\begin{array}{r}\text { TOL 65 No.2 } \\
(65 . \mathrm{N}-0642(\mathrm{~A}))\end{array}$ & $\begin{array}{r}\text { NGC 6822V No.1 } \\
(69 . \mathrm{C}-0203(\mathrm{~A}))\end{array}$ \\
\hline $4740[\mathrm{Ar}$ IV] & $1.90 \pm 0.05$ & $0.62 \pm 0.03$ & $0.55 \pm 0.13$ & $0.49 \pm 0.06$ \\
\hline $4861 \mathrm{H} \beta$ & $100.00 \pm 1.44$ & $100.00 \pm 1.44$ & $100.00 \pm 1.48$ & $100.00 \pm 1.46$ \\
\hline $4921 \mathrm{He}_{\mathrm{I}}$ & $0.88 \pm 0.03$ & $0.93 \pm 0.04$ & $0.45 \pm 0.08$ & $1.16 \pm 0.07$ \\
\hline 4959 [O III] & $175.70 \pm 2.52$ & $125.90 \pm 1.81$ & $114.62 \pm 1.68$ & $186.33 \pm 2.70$ \\
\hline 4988 [Fe III] & $0.53 \pm 0.03$ & $0.43 \pm 0.03$ & $1.24 \pm 0.10$ & \\
\hline 5007 [O III] & $525.94 \pm 7.54$ & $375.89 \pm 5.40$ & $341.57 \pm 4.97$ & $582.55 \pm 8.42$ \\
\hline $5015 \mathrm{He}_{\mathrm{I}}$ & $0.64 \pm 0.01$ & $1.30 \pm 0.04$ & $0.79 \pm 0.09$ & $1.95 \pm 0.07$ \\
\hline $5518[\mathrm{Cl} \mathrm{III}]$ & $\ldots$ & $\ldots$ & $\ldots$ & $0.49 \pm 0.06$ \\
\hline $5538[\mathrm{Cl} \mathrm{III}]$ & $\ldots$ & $0.08 \pm 0.00$ & $\ldots$ & $0.38 \pm 0.06$ \\
\hline $5876 \mathrm{He} \mathrm{I}$ & $8.80 \pm 0.14$ & $9.83 \pm 0.15$ & $9.37 \pm 0.20$ & $11.47 \pm 0.20$ \\
\hline $6300[\mathrm{O} \mathrm{I}]$ & $0.71 \pm 0.03$ & $1.54 \pm 0.03$ & $\ldots$ & $\ldots$ \\
\hline $6312\left[\mathrm{~S}_{\mathrm{III}}\right]$ & $0.69 \pm 0.03$ & $1.06 \pm 0.03$ & $1.48 \pm 0.11$ & $1.93 \pm 0.06$ \\
\hline 6363 [O I $]$ & $0.27 \pm 0.03$ & $0.55 \pm 0.02$ & $\ldots$ & $\ldots$ \\
\hline 6548 [N II] & $0.32 \pm 0.02$ & $0.54 \pm 0.03$ & $\ldots$ & $0.96 \pm 0.04$ \\
\hline $6563 \mathrm{H} \alpha$ & $275.12 \pm 4.28$ & $277.28 \pm 4.32$ & $277.06 \pm 4.38$ & $286.65 \pm 4.50$ \\
\hline $6583[\mathrm{~N} \mathrm{II]}$ & $0.83 \pm 0.03$ & $1.64 \pm 0.04$ & $2.38 \pm 0.11$ & $3.07 \pm 0.08$ \\
\hline $6678 \mathrm{He}_{\mathrm{I}}$ & $2.52 \pm 0.05$ & $2.70 \pm 0.05$ & $2.63 \pm 0.12$ & $3.18 \pm 0.08$ \\
\hline 6717 [S II] & $1.75 \pm 0.04$ & $4.80 \pm 0.08$ & $6.75 \pm 0.16$ & $4.08 \pm 0.09$ \\
\hline $6731\left[\mathrm{~S}_{\mathrm{II}}\right]$ & $1.46 \pm 0.04$ & $3.80 \pm 0.07$ & $4.63 \pm 0.14$ & $3.03 \pm 0.08$ \\
\hline $7065 \mathrm{He}_{\mathrm{I}}$ & $2.58 \pm 0.05$ & $2.94 \pm 0.06$ & $2.23 \pm 0.11$ & $2.83 \pm 0.07$ \\
\hline $7136[\mathrm{Ar}$ III] & $\ldots$ & $2.79 \pm 0.05$ & $3.22 \pm 0.11$ & $9.62 \pm 0.17$ \\
\hline $7281 \mathrm{He}$ I & $\ldots$ & $\ldots$ & $\ldots$ & $0.63 \pm 0.03$ \\
\hline 7320 [O II] & $\ldots$ & $\ldots$ & $\ldots$ & $1.26 \pm 0.05$ \\
\hline 7330 [O II] & $\ldots$ & $\ldots$ & $\ldots$ & $0.92 \pm 0.04$ \\
\hline$C(\mathrm{H} \beta)$ & 0.250 & 0.250 & 0.180 & 0.545 \\
\hline$F(\mathrm{H} \beta)^{a}$ & 111.80 & 150.80 & 35.64 & 66.21 \\
\hline$E W(\mathrm{H} \beta) \AA$ & 317.9 & 278.1 & 76.1 & 140.3 \\
\hline$E W($ abs $) \AA$ & 0.75 & 2.80 & 0.15 & 0.05 \\
\hline \multirow[b]{2}{*}{ Line } & \multicolumn{4}{|c|}{$\begin{array}{c}\text { GALAXY } \\
\text { (ESO PROGRAM) }\end{array}$} \\
\hline & NGC 6822V No.2 & NGC 6822X & TOL2138-405 No.1 & TOL2138-405 No.3 \\
\hline $3727[\mathrm{O}$ II] & $\frac{(09 .(-0205(\mathrm{~A}))}{91.55 \pm 1.66}$ & $\frac{09 .(-0203(\mathrm{~A}))}{111.50+2.71}$ & $\frac{09 .(-0203(A))}{128.57+2.04}$ & $\frac{09 .(-0203(\mathrm{~A}))}{166.91+2.88}$ \\
\hline $3750 \mathrm{H} 12$ & $3.62 \pm 0.51$ & $\ldots$ & $3.41 \pm 0.16$ & $2.81 \pm 0.39$ \\
\hline 3771 H11 & $4.97 \pm 0.51$ & $\ldots$ & $4.08 \pm 0.17$ & $3.98 \pm 0.42$ \\
\hline 3798 H10 & $5.88 \pm 0.49$ & $\ldots$ & $5.49 \pm 0.17$ & $5.41 \pm 0.41$ \\
\hline $3820 \mathrm{He}_{\mathrm{I}}$ & $1.77 \pm 0.25$ & $\ldots$ & $1.19 \pm 0.09$ & $\ldots$ \\
\hline $3835 \mathrm{H} 9$ & $8.15 \pm 0.48$ & $11.81 \pm 1.87$ & $7.85 \pm 0.20$ & $7.70 \pm 0.43$ \\
\hline 3868 [Ne III] & $47.47 \pm 0.89$ & $37.02 \pm 1.25$ & $56.38 \pm 0.89$ & $46.12 \pm 0.89$ \\
\hline 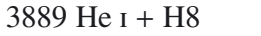 & $20.77 \pm 0.56$ & $19.91 \pm 1.25$ & $20.50 \pm 0.36$ & $19.85 \pm 0.56$ \\
\hline 3968 [Ne III] + H7 & $29.68 \pm 0.66$ & $28.58 \pm 1.29$ & $33.09 \pm 0.54$ & $29.96 \pm 0.70$ \\
\hline $4026 \mathrm{He}_{\mathrm{I}}$ & $1.91 \pm 0.21$ & $\ldots$ & $1.76 \pm 0.08$ & $1.74 \pm 0.23$ \\
\hline 4068 [S II] & $\ldots$ & $\ldots$ & $1.56 \pm 0.08$ & $1.97 \pm 0.23$ \\
\hline $4076\left[\mathrm{~S}_{\mathrm{II}}\right]$ & $\ldots$ & $\ldots$ & $0.50 \pm 0.06$ & $\ldots$ \\
\hline $4101 \mathrm{H} \delta$ & $26.16 \pm 0.57$ & $27.47 \pm 1.08$ & $26.46 \pm 0.43$ & $26.83 \pm 0.60$ \\
\hline $4340 \mathrm{H} \gamma$ & $46.47 \pm 0.79$ & $45.77 \pm 1.12$ & $48.78 \pm 0.74$ & $48.08 \pm 0.86$ \\
\hline 4363 [O III] & $6.63 \pm 0.20$ & $5.68 \pm 0.46$ & $10.48 \pm 0.18$ & $9.87 \pm 0.31$ \\
\hline $4387 \mathrm{He}_{\mathrm{I}}$ & $\ldots$ & $\ldots$ & $0.49 \pm 0.06$ & $\ldots$ \\
\hline $4471 \mathrm{He} \mathrm{I}$ & $3.90 \pm 0.16$ & $3.29 \pm 0.37$ & $3.85 \pm 0.09$ & $3.41 \pm 0.23$ \\
\hline $4658[\mathrm{Fe} \mathrm{III}]$ & $\ldots$ & $\ldots$ & $0.91 \pm 0.06$ & $1.17 \pm 0.19$ \\
\hline $4686 \mathrm{He}_{\text {II }}$ & $\ldots$ & $\ldots$ & $1.06 \pm 0.06$ & $2.60 \pm 0.21$ \\
\hline $4711[\mathrm{Ar}$ IV $]+\mathrm{He}_{\mathrm{I}}$ & $1.06 \pm 0.12$ & $\ldots$ & $1.75 \pm 0.07$ & $1.68 \pm 0.20$ \\
\hline 4740 [Ar IV] & $0.55 \pm 0.12$ & $\ldots$ & $1.02 \pm 0.05$ & $\ldots$ \\
\hline $4861 \mathrm{H} \beta$ & $100.00 \pm 1.52$ & $100.00 \pm 1.81$ & $100.00 \pm 1.46$ & $100.00 \pm 1.58$ \\
\hline $4921 \mathrm{He} \mathrm{I}$ & $1.55 \pm 0.13$ & $1.10 \pm 0.28$ & $0.89 \pm 0.06$ & $\ldots$ \\
\hline 4959 [O III] & $183.83 \pm 2.75$ & $162.31 \pm 2.77$ & $212.82 \pm 3.08$ & $187.88 \pm 2.89$ \\
\hline 4988 [Fe III] & $\ldots$ & $\ldots$ & $0.95 \pm 0.05$ & $1.86 \pm 0.17$ \\
\hline $5007[\mathrm{O} \mathrm{III]}$ & $575.53 \pm 8.52$ & $464.54 \pm 7.67$ & $644.03 \pm 9.29$ & $553.22 \pm 8.38$ \\
\hline $5015 \mathrm{He}_{\mathrm{I}}$ & $2.48 \pm 0.13$ & $2.40 \pm 0.29$ & $1.26 \pm 0.05$ & $1.60 \pm 0.14$ \\
\hline $5518[\mathrm{Cl} \mathrm{III}]$ & $0.45 \pm 0.11$ & $\ldots$ & $0.43 \pm 0.06$ & $\ldots$ \\
\hline 5538 [Cl III] & $0.41 \pm 0.07$ & $\ldots$ & $0.28 \pm 0.05$ & $\ldots$ \\
\hline $5755[\mathrm{~N}$ II $]$ & $\ldots$ & $\ldots$ & $0.27 \pm 0.05$ & $\ldots$ \\
\hline
\end{tabular}


Table 5. continued.

\begin{tabular}{|c|c|c|c|c|}
\hline \multirow[b]{2}{*}{ Line } & \multicolumn{4}{|c|}{$\begin{array}{c}\text { Galaxy } \\
\text { (ESO PROGRAM) }\end{array}$} \\
\hline & $\begin{array}{r}\text { NGC 6822V No.2 } \\
(69 . C-0203(\mathrm{~A}))\end{array}$ & $\begin{array}{r}\text { NGC 6822X } \\
(69 . C-0203(A))\end{array}$ & $\begin{array}{r}\text { TOL2138-405 No.1 } \\
(69 . \mathrm{C}-0203(\mathrm{~A}))\end{array}$ & $\begin{array}{r}\text { TOL2138-405 No.3 } \\
(69 . \mathrm{C}-0203(\mathrm{~A}))\end{array}$ \\
\hline $5876 \mathrm{He} \mathrm{I}$ & $11.07 \pm 0.25$ & $11.13 \pm 0.44$ & $11.58 \pm 0.19$ & $10.83 \pm 0.26$ \\
\hline $6300\left[\mathrm{O}_{\mathrm{I}}\right]$ & $\ldots$ & $\ldots$ & $3.14 \pm 0.07$ & $3.64 \pm 0.16$ \\
\hline $6312\left[\mathrm{~S}_{\text {III }}\right]$ & $1.91 \pm 0.10$ & $1.77 \pm 0.30$ & $1.89 \pm 0.05$ & $1.66 \pm 0.15$ \\
\hline $6363[\mathrm{O} \mathrm{I}]$ & $\ldots$ & $\ldots$ & $0.95 \pm 0.04$ & $1.12 \pm 0.12$ \\
\hline $6548[\mathrm{~N} \mathrm{II}]$ & $1.65 \pm 0.08$ & $1.97 \pm 0.30$ & $3.87 \pm 0.08$ & $1.98 \pm 0.14$ \\
\hline $6563 \mathrm{H} \alpha$ & $286.39 \pm 4.63$ & $284.63 \pm 5.20$ & $282.29 \pm 4.42$ & $281.43 \pm 4.65$ \\
\hline 6583 [N II] & $3.97 \pm 0.13$ & $5.26 \pm 0.35$ & $12.55 \pm 0.21$ & $5.71 \pm 0.19$ \\
\hline $6678 \mathrm{He}_{\mathrm{I}}$ & $3.10 \pm 0.10$ & $2.96 \pm 0.28$ & $3.12 \pm 0.07$ & $2.90 \pm 0.17$ \\
\hline 6717 [S II] & $5.62 \pm 0.15$ & $7.25 \pm 0.32$ & $10.68 \pm 0.18$ & $13.86 \pm 0.31$ \\
\hline 6731 [S II] & $3.82 \pm 0.11$ & $3.98 \pm 0.25$ & $9.38 \pm 0.16$ & $10.07 \pm 0.26$ \\
\hline $7065 \mathrm{He} \mathrm{I}$ & $2.29 \pm 0.11$ & $2.54 \pm 0.26$ & $3.40 \pm 0.08$ & $2.00 \pm 0.14$ \\
\hline 7136 [Ar III] & $9.24 \pm 0.20$ & $10.05 \pm 0.38$ & $\ldots$ & $\ldots$ \\
\hline $7281 \mathrm{He} \mathrm{I}$ & $0.63 \pm 0.07$ & $\ldots$ & $\ldots$ & $\ldots$ \\
\hline 7320 [O II] & $1.15 \pm 0.10$ & $\ldots$ & $\ldots$ & $\ldots$ \\
\hline 7330 [O II] & $1.05 \pm 0.09$ & $\ldots$ & $\ldots$ & $\ldots$ \\
\hline$C(\mathrm{H} \beta)$ & 0.655 & 0.255 & 0.290 & 0.265 \\
\hline$F(\mathrm{H} \beta)^{a}$ & 23.10 & 7.61 & 58.06 & 11.78 \\
\hline$E W(\mathrm{H} \beta) \AA$ & 131.1 & 54.6 & 265.9 & 165.3 \\
\hline$E W($ abs $) \AA$ & 0.40 & 1.70 & 0.50 & 0.05 \\
\hline \multirow{3}{*}{ Line } & \multicolumn{4}{|c|}{$\begin{array}{l}\text { GaLAXY } \\
\text { ESO PROGRAM) }\end{array}$} \\
\hline & TOL2146-391 No.1 & TOL2146-391 No.2 & TOL 2240-384 & UM 160 No.1 \\
\hline & (69.C-0203(A)) & (69.C-0203(A)) & (69.C-0203(A)) & (69.C-0203(A)) \\
\hline 3727 [O II] & $73.86 \pm 1.18$ & $71.21 \pm 1.17$ & $43.91 \pm 0.74$ & $123.51 \pm 2.10$ \\
\hline $3750 \mathrm{H} 12$ & $3.48 \pm 0.15$ & $3.55 \pm 0.23$ & $3.11 \pm 0.21$ & $5.24 \pm 0.46$ \\
\hline 3771 H11 & $4.59 \pm 0.16$ & $4.50 \pm 0.23$ & $3.61 \pm 0.19$ & $6.61 \pm 0.47$ \\
\hline 3798 H10 & $5.57 \pm 0.17$ & $5.46 \pm 0.22$ & $4.24 \pm 0.18$ & $7.62 \pm 0.41$ \\
\hline $3820 \mathrm{He}_{\mathrm{I}}$ & $0.80 \pm 0.07$ & $0.75 \pm 0.11$ & $0.71 \pm 0.07$ & $1.16 \pm 0.14$ \\
\hline 3835 H9 & $7.58 \pm 0.18$ & $7.66 \pm 0.23$ & $5.48 \pm 0.19$ & $9.41 \pm 0.38$ \\
\hline 3868 [Ne III] & $44.72 \pm 0.71$ & $44.42 \pm 0.73$ & $32.69 \pm 0.55$ & $38.66 \pm 0.70$ \\
\hline $3889 \mathrm{He}_{\mathrm{I}}+\mathrm{H} 8$ & $19.92 \pm 0.34$ & $20.04 \pm 0.38$ & $11.64 \pm 0.25$ & $20.23 \pm 0.46$ \\
\hline $3968[\mathrm{Ne}$ III $]+\mathrm{H} 7$ & $29.60 \pm 0.48$ & $29.98 \pm 0.51$ & $21.62 \pm 0.38$ & $30.08 \pm 0.59$ \\
\hline $4026 \mathrm{He}_{\mathrm{I}}$ & $1.67 \pm 0.07$ & $1.56 \pm 0.11$ & $1.16 \pm 0.07$ & $1.38 \pm 0.11$ \\
\hline 4068 [S II] & $0.92 \pm 0.06$ & $0.81 \pm 0.09$ & $0.78 \pm 0.06$ & $1.13 \pm 0.11$ \\
\hline 4076 [S II] & $0.24 \pm 0.04$ & $0.29 \pm 0.07$ & $0.28 \pm 0.05$ & $0.48 \pm 0.08$ \\
\hline $4101 \mathrm{H} \delta$ & $25.95 \pm 0.41$ & $26.39 \pm 0.44$ & $18.26 \pm 0.32$ & $26.73 \pm 0.51$ \\
\hline $4227[\mathrm{Fe} \mathrm{v}]$ & $0.68 \pm 0.16$ & $0.78 \pm 0.24$ & $2.02 \pm 0.56$ & $\ldots$ \\
\hline $4340 \mathrm{H} \gamma$ & $47.16 \pm 0.71$ & $47.88 \pm 0.73$ & $37.70 \pm 0.59$ & $45.38 \pm 0.75$ \\
\hline 4363 [O III] & $12.78 \pm 0.21$ & $12.69 \pm 0.22$ & $11.92 \pm 0.20$ & $9.03 \pm 0.20$ \\
\hline $4387 \mathrm{He} \mathrm{I}$ & $0.51 \pm 0.06$ & $0.35 \pm 0.06$ & $0.36 \pm 0.04$ & $0.47 \pm 0.10$ \\
\hline $4471 \mathrm{He}_{\mathrm{I}}$ & $3.93 \pm 0.09$ & $3.88 \pm 0.11$ & $3.70 \pm 0.08$ & $3.66 \pm 0.12$ \\
\hline $4658[\mathrm{Fe}$ III] & $0.61 \pm 0.04$ & $0.54 \pm 0.07$ & $0.63 \pm 0.05$ & $0.68 \pm 0.07$ \\
\hline $4686 \mathrm{He}_{\text {II }}$ & $1.73 \pm 0.05$ & $2.30 \pm 0.09$ & $1.38 \pm 0.06$ & $0.34 \pm 0.05$ \\
\hline 4711 [Ar IV] + He I & $3.06 \pm 0.07$ & $\ldots$ & $2.07 \pm 0.06$ & $1.08 \pm 0.08$ \\
\hline 4740 [Ar IV] & $1.19 \pm 0.05$ & $1.13 \pm 0.07$ & $1.40 \pm 0.05$ & $0.47 \pm 0.06$ \\
\hline $4861 \mathrm{H} \beta$ & $100.00 \pm 1.45$ & $100.00 \pm 1.46$ & $100.00 \pm 1.49$ & $100.00 \pm 1.52$ \\
\hline $4921 \mathrm{He}_{\mathrm{I}}$ & $0.90 \pm 0.04$ & $0.94 \pm 0.06$ & $0.90 \pm 0.04$ & $0.92 \pm 0.07$ \\
\hline 4959 [O III] & $199.39 \pm 2.88$ & $192.55 \pm 2.80$ & $218.33 \pm 3.24$ & $177.38 \pm 2.66$ \\
\hline 4988 [Fe III] & $0.92 \pm 0.04$ & $0.96 \pm 0.05$ & $0.75 \pm 0.06$ & $0.74 \pm 0.07$ \\
\hline 5007 [O III] & $587.08 \pm 8.46$ & $564.63 \pm 8.17$ & $663.42 \pm 9.81$ & $541.18 \pm 8.07$ \\
\hline 5015 He I & $1.39 \pm 0.04$ & $1.44 \pm 0.07$ & $\ldots$ & $1.38 \pm 0.07$ \\
\hline 5518 [Cl III] & $0.25 \pm 0.03$ & $0.22 \pm 0.04$ & $0.32 \pm 0.03$ & $0.33 \pm 0.05$ \\
\hline 5538 [Cl III] & $0.14 \pm 0.03$ & $0.15 \pm 0.04$ & $\ldots$ & $0.32 \pm 0.05$ \\
\hline 5755 [N II] & $\ldots$ & $\ldots$ & $0.38 \pm 0.05$ & $\ldots$ \\
\hline $5876 \mathrm{He}_{\mathrm{I}}$ & $10.60 \pm 0.17$ & $10.65 \pm 0.18$ & $12.51 \pm 0.22$ & $10.11 \pm 0.19$ \\
\hline $6300[\mathrm{O} I]$ & $2.13 \pm 0.05$ & $1.83 \pm 0.06$ & $2.01 \pm 0.06$ & $2.23 \pm 0.07$ \\
\hline $6312[\mathrm{~S}$ III] & $1.51 \pm 0.04$ & $1.45 \pm 0.05$ & $0.88 \pm 0.04$ & $1.79 \pm 0.06$ \\
\hline 6363 [O I] & $0.68 \pm 0.03$ & $0.58 \pm 0.04$ & $0.62 \pm 0.04$ & $0.76 \pm 0.04$ \\
\hline $6548[\mathrm{~N} \mathrm{II}]$ & $0.98 \pm 0.04$ & $0.68 \pm 0.04$ & $\ldots$ & $1.25 \pm 0.06$ \\
\hline $6563 \mathrm{H} \alpha$ & $279.90 \pm 4.38$ & $279.53 \pm 4.39$ & $284.13 \pm 4.56$ & $282.93 \pm 4.58$ \\
\hline 6583 [N II] & $2.90 \pm 0.06$ & $2.38 \pm 0.06$ & $5.68 \pm 0.12$ & $4.08 \pm 0.09$ \\
\hline $6678 \mathrm{He}_{\mathrm{I}}$ & $2.78 \pm 0.06$ & $2.90 \pm 0.07$ & $2.77 \pm 0.07$ & $2.94 \pm 0.08$ \\
\hline
\end{tabular}


Table 5. continued.

\begin{tabular}{|c|c|c|c|c|}
\hline \multirow[b]{2}{*}{ Line } & \multicolumn{4}{|c|}{$\begin{array}{c}\text { GALAXY } \\
\text { (ESO PROGRAM) }\end{array}$} \\
\hline & $\begin{array}{r}\text { TOL2146-391 No.1 } \\
(69 . \mathrm{C}-0203(\mathrm{~A}))\end{array}$ & $\begin{array}{r}\text { TOL2146-391 No.2 } \\
(69 . \mathrm{C}-0203(\mathrm{~A}))\end{array}$ & $\begin{array}{l}\text { TOL 2240-384 } \\
(69 . \mathrm{C}-0203(\mathrm{~A}))\end{array}$ & $\begin{array}{r}\text { UM 160 No.1 } \\
(69 . \mathrm{C}-0203(\mathrm{~A}))\end{array}$ \\
\hline $6717[\mathrm{~S} \mathrm{II}]$ & $6.87 \pm 0.12$ & $5.68 \pm 0.11$ & $4.10 \pm 0.09$ & $9.23 \pm 0.17$ \\
\hline 6731 [S II] & $5.43 \pm 0.10$ & $4.45 \pm 0.09$ & $3.56 \pm 0.08$ & $7.17 \pm 0.14$ \\
\hline $7065 \mathrm{He}_{\mathrm{I}}$ & $2.92 \pm 0.06$ & $2.91 \pm 0.07$ & $6.01 \pm 0.13$ & $2.45 \pm 0.07$ \\
\hline 7136 [Ar III] & $4.93 \pm 0.09$ & $4.32 \pm 0.09$ & $\ldots$ & $5.54 \pm 0.12$ \\
\hline $7281 \mathrm{He}$ I & $\ldots$ & $\ldots$ & $\ldots$ & $0.63 \pm 0.04$ \\
\hline $7320[\mathrm{O}$ II] & $\ldots$ & $\ldots$ & $\ldots$ & $1.63 \pm 0.06$ \\
\hline $7330[\mathrm{O}$ II] & $\ldots$ & $\ldots$ & $\ldots$ & $1.61 \pm 0.06$ \\
\hline$C(\mathrm{H} \beta)$ & 0.320 & 0.305 & 0.830 & 0.460 \\
\hline$F(\mathrm{H} \beta)^{a}$ & 77.87 & 54.50 & 79.96 & 33.02 \\
\hline$E W(\mathrm{H} \beta) \AA$ & 315.3 & 221.0 & 284.4 & 246.3 \\
\hline$E W($ abs $) \AA$ & 1.00 & 0.75 & 5.20 & 5.15 \\
\hline \multirow[b]{2}{*}{ Line } & \multicolumn{2}{|c|}{$\begin{array}{c}\text { GALAXY } \\
\text { (ESO PROGRAM) }\end{array}$} & & \\
\hline & $\begin{array}{l}\text { UM 160 No.2 } \\
(69 . \mathrm{C}-0203(\mathrm{~A}))\end{array}$ & $\begin{array}{r}\text { UM } 160 \text { No.3 } \\
\text { (69.C-0203(A)) }\end{array}$ & & \\
\hline 3727 [O II] & $146.49 \pm 2.68$ & $177.17 \pm 3.29$ & & \\
\hline $3750 \mathrm{H} 12$ & $2.71 \pm 0.60$ & $8.20 \pm 1.67$ & & \\
\hline 3771 H11 & $4.37 \pm 0.61$ & $8.76 \pm 1.74$ & & \\
\hline 3798 H10 & $5.00 \pm 0.58$ & $9.52 \pm 1.14$ & & \\
\hline $3835 \mathrm{H} 9$ & $6.50 \pm 0.60$ & $12.25 \pm 1.08$ & & \\
\hline 3868 [Ne III] & $40.24 \pm 0.89$ & $30.96 \pm 0.79$ & & \\
\hline $3889 \mathrm{He} \mathrm{I}_{+} \mathrm{H} 8$ & $19.15 \pm 0.66$ & $22.61 \pm 0.87$ & & \\
\hline 3968 [Ne III] + H7 & $27.54 \pm 0.76$ & $27.82 \pm 0.89$ & & \\
\hline $4026 \mathrm{He}$ I & $\ldots$ & $1.80 \pm 0.29$ & & \\
\hline 4068 [S II] & $\ldots$ & $2.14 \pm 0.28$ & & \\
\hline 4076 [S II] & $\ldots$ & $0.85 \pm 0.25$ & & \\
\hline $4101 \mathrm{H} \delta$ & $25.58 \pm 0.67$ & $27.66 \pm 0.80$ & & \\
\hline $4340 \mathrm{H} \gamma$ & $45.06 \pm 0.88$ & $45.45 \pm 0.78$ & & \\
\hline 4363 [O III] & $6.26 \pm 0.29$ & $4.29 \pm 0.07$ & & \\
\hline $4471 \mathrm{He}_{\mathrm{I}}$ & $3.40 \pm 0.22$ & $3.59 \pm 0.06$ & & \\
\hline 4686 He II & $0.80 \pm 0.16$ & $\ldots$ & & \\
\hline $4711\left[\right.$ Ar IV] $+\mathrm{He}_{\mathrm{I}}$ & $0.85 \pm 0.17$ & $\ldots$ & & \\
\hline $4861 \mathrm{H} \beta$ & $100.00 \pm 1.63$ & $100.00 \pm 1.66$ & & \\
\hline $4921 \mathrm{He}_{\mathrm{I}}$ & & $0.93 \pm 0.15$ & & \\
\hline 4959 [O III] & $170.25 \pm 2.65$ & $137.10 \pm 2.21$ & & \\
\hline 4988 [Fe III] & $0.56 \pm 0.15$ & $0.96 \pm 0.15$ & & \\
\hline 5007 [O III] & $520.03 \pm 7.93$ & $418.88 \pm 6.60$ & & \\
\hline $5015 \mathrm{He} \mathrm{I}$ & $1.66 \pm 0.17$ & $1.64 \pm 0.16$ & & \\
\hline $5876 \mathrm{He}$ I & $11.26 \pm 0.29$ & $10.78 \pm 0.27$ & & \\
\hline 6300 [O I] & $1.87 \pm 0.14$ & $3.39 \pm 0.15$ & & \\
\hline 6312 [S III] & $1.86 \pm 0.13$ & $1.94 \pm 0.13$ & & \\
\hline 6363 [O I] & $0.82 \pm 0.17$ & $0.89 \pm 0.11$ & & \\
\hline 6548 [N II] & $2.12 \pm 0.14$ & $3.25 \pm 0.14$ & & \\
\hline $6563 \mathrm{H} \alpha$ & $283.43 \pm 4.74$ & $286.00 \pm 4.90$ & & \\
\hline 6583 [N II] & $6.01 \pm 0.20$ & $8.79 \pm 0.22$ & & \\
\hline $6678 \mathrm{He} \mathrm{I}$ & $2.89 \pm 0.17$ & $2.91 \pm 0.13$ & & \\
\hline 6717 [S II] & $12.71 \pm 0.32$ & $17.63 \pm 0.37$ & & \\
\hline 6731 [S II] & $8.59 \pm 0.25$ & $12.44 \pm 0.28$ & & \\
\hline $7065 \mathrm{He}$ I & $2.00 \pm 0.14$ & $1.91 \pm 0.12$ & & \\
\hline 7136 [Ar III] & $5.68 \pm 0.20$ & $7.22 \pm 0.19$ & & \\
\hline $7281 \mathrm{He}$ I & $\ldots$ & $0.49 \pm 0.08$ & & \\
\hline 7320 [O II] & $1.40 \pm 0.16$ & $2.69 \pm 0.13$ & & \\
\hline 7330 [O II] & $1.84 \pm 0.15$ & $1.94 \pm 0.13$ & & \\
\hline$C(\mathrm{H} \beta)$ & 0.000 & 0.315 & & \\
\hline$F(\mathrm{H} \beta)^{a}$ & 11.53 & 12.10 & & \\
\hline$E W(\mathrm{H} \beta) \AA$ & 90.2 & 164.8 & & \\
\hline$E W($ abs $) \AA$ & 0.00 & 5.60 & & \\
\hline
\end{tabular}

Notes. ${ }^{(a)}$ In units $10^{-16} \mathrm{erg} \mathrm{s}^{-1} \mathrm{~cm}^{-2}$. 
Table 6. Ionic and total heavy element abundances (UVES observations).

\begin{tabular}{|c|c|c|c|c|}
\hline \multirow[b]{2}{*}{ Property } & \multicolumn{4}{|c|}{$\begin{array}{c}\text { GALAXY } \\
\text { (ESO PROGRAM) }\end{array}$} \\
\hline & $\begin{array}{c}\text { UM 254 } \\
(71 . \mathrm{B}-0055(\mathrm{~A}))\end{array}$ & $\begin{array}{c}\text { UM 283D } \\
(71 . \mathrm{B}-0055(\mathrm{~A}))\end{array}$ & $\begin{array}{c}\text { UM 283O } \\
(70 . \mathrm{B}-0717(\mathrm{~A}))\end{array}$ & $\begin{array}{c}\text { UM 133H } \\
(68 . \mathrm{B}-0310(\mathrm{~A}))\end{array}$ \\
\hline$T_{\mathrm{e}}(\mathrm{O}$ III $)(\mathrm{K})$ & $10666 \pm 260$ & $12360 \pm 338$ & $11761 \pm 293$ & $15504 \pm 243$ \\
\hline$T_{\mathrm{e}}(\mathrm{O}$ ІІ $)(\mathrm{K})$ & $10666 \pm 287$ & $12139 \pm 381$ & $12082 \pm 328$ & $14146 \pm 287$ \\
\hline$T_{\mathrm{e}}(\mathrm{S}$ III) $(\mathrm{K})$ & $10673 \pm 215$ & $11591 \pm 281$ & $11639 \pm 243$ & $15124 \pm 202$ \\
\hline$N_{\mathrm{e}}(\mathrm{S}$ II $)\left(\mathrm{cm}^{-3}\right)$ & $10 \pm 10$ & $15 \pm 15$ & $10 \pm 10$ & $59 \pm 40$ \\
\hline $\mathrm{O}^{+} / \mathrm{H}^{+}\left(\times 10^{4}\right)$ & $0.77 \pm 0.08$ & $0.55 \pm 0.06$ & $0.45 \pm 0.04$ & $0.12 \pm 0.01$ \\
\hline $\mathrm{O}^{++} / \mathrm{H}^{+}\left(\times 10^{4}\right)$ & $0.67 \pm 0.05$ & $0.63 \pm 0.05$ & $0.70 \pm 0.05$ & $0.38 \pm 0.02$ \\
\hline $\mathrm{O} / \mathrm{H}\left(\times 10^{4}\right)$ & $1.44 \pm 0.09$ & $1.18 \pm 0.08$ & $1.14 \pm 0.07$ & $0.49 \pm 0.02$ \\
\hline $12+\log (\mathrm{O} / \mathrm{H})$ & $8.16 \pm 0.03$ & $8.07 \pm 0.03$ & $8.06 \pm 0.03$ & $7.69 \pm 0.02$ \\
\hline $\mathrm{N}^{+} / \mathrm{H}^{+}\left(\times 10^{6}\right)$ & $5.92 \pm 0.61$ & $2.31 \pm 0.25$ & $2.30 \pm 0.22$ & $0.26 \pm 0.02$ \\
\hline ICF & $2.21 \pm 0.18$ & $2.43 \pm 0.21$ & $2.81 \pm 0.21$ & $4.11 \pm 0.24$ \\
\hline $\log (\mathrm{N} / \mathrm{O})$ & $-1.04 \pm 0.06$ & $-1.32 \pm 0.07$ & $-1.25 \pm 0.06$ & $-1.67 \pm 0.04$ \\
\hline $\mathrm{Ne}^{++} / \mathrm{H}^{+}\left(\times 10^{5}\right)$ & $1.88 \pm 0.17$ & $1.35 \pm 0.12$ & $1.46 \pm 0.12$ & $0.69 \pm 0.03$ \\
\hline ICF & $1.75 \pm 0.11$ & $1.48 \pm 0.09$ & $1.35 \pm 0.07$ & $1.11 \pm 0.02$ \\
\hline $\log (\mathrm{Ne} / \mathrm{O})$ & $-0.64 \pm 0.06$ & $-0.77 \pm 0.05$ & $-0.76 \pm 0.05$ & $-0.81 \pm 0.03$ \\
\hline $\mathrm{S}^{+} / \mathrm{H}^{+}\left(\times 10^{6}\right)$ & $\ldots$ & $0.72 \pm 0.04$ & $\cdots$ & $0.18 \pm 0.01$ \\
\hline $\mathrm{S}^{++} / \mathrm{H}^{+}\left(\times 10^{6}\right)$ & $\ldots$ & $2.25 \pm 0.23$ & $\ldots$ & $0.77 \pm 0.05$ \\
\hline ICF & $\ldots$ & $1.03 \pm 0.04$ & $\ldots$ & $1.16 \pm 0.02$ \\
\hline $\log (\mathrm{S} / \mathrm{O})$ & $\ldots$ & $-1.58 \pm 0.05$ & $\ldots$ & $-1.65 \pm 0.03$ \\
\hline $\mathrm{Ar}^{++} / \mathrm{H}^{+}\left(\times 10^{7}\right)$ & $5.52 \pm 0.28$ & $6.15 \pm 0.35$ & $\cdots$ & $1.72 \pm 0.06$ \\
\hline $\mathrm{Ar}^{+++} / \mathrm{H}^{+}\left(\times 10^{7}\right)$ & & & $\ldots$ & \\
\hline ICF & $1.11 \pm 0.03$ & $1.09 \pm 0.03$ & $\ldots$ & $1.11 \pm 0.02$ \\
\hline $\log (\mathrm{Ar} / \mathrm{O})$ & $-2.37 \pm 0.04$ & $-2.25 \pm 0.04$ & $\cdots$ & $-2.41 \pm 0.02$ \\
\hline $\mathrm{Fe}^{++} / \mathrm{H}^{+}\left(\times 10^{6}\right)(4658)$ & $1.37 \pm 0.15$ & $\cdots$ & $\cdots$ & $\cdots$ \\
\hline & $2.79 \pm 0.26$ & $\ldots$ & $\ldots$ & $\ldots$ \\
\hline $\log (\mathrm{Fe} / \mathrm{O})(\lambda 4658)$ & $-1.58 \pm 0.07$ & $\ldots$ & $\ldots$ & $\ldots$ \\
\hline \multirow{3}{*}{ Property } & \multicolumn{4}{|c|}{$\begin{array}{c}\text { GaLAXY } \\
\text { (ESO PROGRAM) }\end{array}$} \\
\hline & UM $133 \mathrm{O}$ & UM 382 & UM 408 & UM 417 \\
\hline & $(70 . \mathrm{B}-0717(\mathrm{~A}))$ & $(70 . \mathrm{B}-0717(\mathrm{~A}))$ & (70.B-0717(A)) & $(70 . \mathrm{B}-0717(\mathrm{~A}))$ \\
\hline$T_{\mathrm{e}}(\mathrm{O}$ III $)(\mathrm{K})$ & $15415 \pm 177$ & $15616 \pm 141$ & $13655 \pm 147$ & $17086 \pm 177$ \\
\hline$T_{\mathrm{e}}\left(\mathrm{O}_{\text {II }}\right)(\mathrm{K})$ & $14106 \pm 209$ & $14195 \pm 167$ & $13111 \pm 169$ & $14699 \pm 214$ \\
\hline$T_{\mathrm{e}}(\mathrm{S}$ III) $(\mathrm{K})$ & $15039 \pm 147$ & $15230 \pm 117$ & $13176 \pm 122$ & $16493 \pm 147$ \\
\hline$N_{\mathrm{e}}(\mathrm{S}$ II $)\left(\mathrm{cm}^{-3}\right)$ & $34 \pm 33$ & $10 \pm 10$ & $10 \pm 10$ & $83 \pm 42$ \\
\hline $\mathrm{O}^{+} / \mathrm{H}^{+}\left(\times 10^{4}\right)$ & $0.10 \pm 0.00$ & $0.05 \pm 0.00$ & $0.15 \pm 0.01$ & $0.06 \pm 0.00$ \\
\hline $\mathrm{O}^{++} / \mathrm{H}^{+}\left(\times 10^{4}\right)$ & $0.42 \pm 0.01$ & $0.60 \pm 0.02$ & $0.73 \pm 0.02$ & $0.41 \pm 0.01$ \\
\hline $\mathrm{O} / \mathrm{H}\left(\times 10^{4}\right)$ & $0.52 \pm 0.01$ & $0.65 \pm 0.02$ & $0.88 \pm 0.02$ & $0.47 \pm 0.01$ \\
\hline $12+\log (\mathrm{O} / \mathrm{H})$ & $7.71 \pm 0.01$ & $7.81 \pm 0.01$ & $7.94 \pm 0.01$ & $7.67 \pm 0.01$ \\
\hline $\mathrm{N}^{+} / \mathrm{H}^{+}\left(\times 10^{6}\right)$ & $0.25 \pm 0.01$ & $0.21 \pm 0.01$ & $0.51 \pm 0.03$ & $0.19 \pm 0.01$ \\
\hline ICF & $4.98 \pm 0.22$ & $11.94 \pm 0.48$ & $5.61 \pm 0.23$ & $7.28 \pm 0.32$ \\
\hline $\log (\mathrm{N} / \mathrm{O})$ & $-1.62 \pm 0.03$ & $-1.42 \pm 0.03$ & $-1.48 \pm 0.03$ & $-1.53 \pm 0.03$ \\
\hline $\mathrm{Ne}^{++} / \mathrm{H}^{+}\left(\times 10^{5}\right)$ & $0.74 \pm 0.03$ & $1.03 \pm 0.03$ & $1.36 \pm 0.05$ & $0.73 \pm 0.02$ \\
\hline ICF & $1.09 \pm 0.02$ & $1.02 \pm 0.02$ & $1.08 \pm 0.02$ & $1.05 \pm 0.01$ \\
\hline $\log (\mathrm{Ne} / \mathrm{O})$ & $-0.81 \pm 0.02$ & $-0.79 \pm 0.02$ & $-0.78 \pm 0.02$ & $-0.79 \pm 0.02$ \\
\hline $\mathrm{S}^{+} / \mathrm{H}^{+}\left(\times 10^{6}\right)$ & $0.17 \pm 0.00$ & $0.06 \pm 0.00$ & $0.23 \pm 0.01$ & $0.11 \pm 0.00$ \\
\hline $\mathrm{S}^{++} / \mathrm{H}^{+}\left(\times 10^{6}\right)$ & $0.78 \pm 0.03$ & $0.91 \pm 0.02$ & $1.27 \pm 0.05$ & $0.56 \pm 0.02$ \\
\hline ICF & $1.22 \pm 0.02$ & $1.91 \pm 0.05$ & $1.37 \pm 0.03$ & $1.39 \pm 0.03$ \\
\hline $\log (\mathrm{S} / \mathrm{O})$ & $-1.65 \pm 0.02$ & $-1.55 \pm 0.02$ & $-1.63 \pm 0.02$ & $-1.71 \pm 0.02$ \\
\hline $\mathrm{Cl}^{++} / \mathrm{H}^{+}\left(\times 10^{8}\right)$ & $\cdots$ & $1.40 \pm 0.06$ & $\ldots$ & $0.68 \pm 0.02$ \\
\hline ICF & $\ldots$ & $2.38 \pm 0.59$ & $\ldots$ & $1.73 \pm 0.42$ \\
\hline $\log (\mathrm{Cl} / \mathrm{O})$ & $\ldots$ & $-3.29 \pm 0.11$ & $\ldots$ & $-3.60 \pm 0.11$ \\
\hline
\end{tabular}


Table 6. continued.

\begin{tabular}{|c|c|c|c|c|}
\hline \multirow[b]{2}{*}{ Property } & \multicolumn{4}{|c|}{$\begin{array}{c}\text { GALAXY } \\
\text { (ESO PROGRAM) }\end{array}$} \\
\hline & $\begin{array}{c}\text { MRK 600 } \\
(70 . \mathrm{B}-0717(\mathrm{~A}))\end{array}$ & $\begin{array}{c}\text { TOL 0618-402 } \\
(70 . \mathrm{B}-0717(\mathrm{~A}))\end{array}$ & $\begin{array}{c}\text { HE 2-10C } \\
(073 . \mathrm{B}-0283(\mathrm{~A}))\end{array}$ & $\begin{array}{c}\text { HE 2-10E } \\
(081 . \mathrm{C}-0113(\mathrm{~A}))\end{array}$ \\
\hline$T_{\mathrm{e}}(\mathrm{O}$ III $)(\mathrm{K})$ & $14990 \pm 154$ & $11413 \pm 191$ & $12415 \pm 218$ & $7450 \pm 355$ \\
\hline$T_{\mathrm{e}}\left(\mathrm{O}_{\text {II }}\right)(\mathrm{K})$ & $13900 \pm 181$ & $11745 \pm 213$ & $12185 \pm 246$ & $9623 \pm 379$ \\
\hline$T_{\mathrm{e}}\left(\mathrm{S}_{\text {III }}\right)(\mathrm{K})$ & $14620 \pm 128$ & $11631 \pm 158$ & $11663 \pm 181$ & $8594 \pm 295$ \\
\hline$N_{\mathrm{e}}(\mathrm{S}$ II $)\left(\mathrm{cm}^{-3}\right)$ & $83 \pm 45$ & $10 \pm 10$ & $1551 \pm 143$ & $1331 \pm 134$ \\
\hline $\mathrm{O}^{+} / \mathrm{H}^{+}\left(\times 10^{4}\right)$ & $0.04 \pm 0.00$ & $0.44 \pm 0.03$ & $0.44 \pm 0.03$ & $1.03 \pm 0.16$ \\
\hline $\mathrm{O}^{++} / \mathrm{H}^{+}\left(\times 10^{4}\right)$ & $0.71 \pm 0.02$ & $1.15 \pm 0.06$ & $0.30 \pm 0.02$ & $1.47 \pm 0.30$ \\
\hline $\mathrm{O}^{+++} / \mathrm{H}^{+}\left(\times 10^{6}\right)$ & $\ldots$ & $\ldots$ & $\ldots$ & $3.47 \pm 0.51$ \\
\hline $\mathrm{O} / \mathrm{H}\left(\times 10^{4}\right)$ & $0.76 \pm 0.02$ & $1.59 \pm 0.07$ & $0.74 \pm 0.03$ & $2.53 \pm 0.34$ \\
\hline $12+\log (\mathrm{O} / \mathrm{H})$ & $7.88 \pm 0.01$ & $8.20 \pm 0.02$ & $7.87 \pm 0.02$ & $8.40 \pm 0.06$ \\
\hline $\mathrm{N}^{+} / \mathrm{H}^{+}\left(\times 10^{6}\right)$ & $0.21 \pm 0.01$ & $1.28 \pm 0.08$ & $10.96 \pm 0.88$ & $15.25 \pm 2.51$ \\
\hline ICF & $14.50 \pm 0.67$ & $3.83 \pm 0.19$ & $1.75 \pm 0.12$ & $2.85 \pm 0.38$ \\
\hline $\log (\mathrm{N} / \mathrm{O})$ & $-1.41 \pm 0.03$ & $-1.51 \pm 0.04$ & $-0.59 \pm 0.05$ & $-0.77 \pm 0.11$ \\
\hline $\mathrm{Ne}^{++} / \mathrm{H}^{+}\left(\times 10^{5}\right)$ & $1.01 \pm 0.03$ & $2.77 \pm 0.17$ & $0.36 \pm 0.02$ & $1.91 \pm 0.46$ \\
\hline ICF & $1.00 \pm 0.02$ & $1.25 \pm 0.06$ & $1.10 \pm 0.05$ & $1.52 \pm 0.24$ \\
\hline $\log (\mathrm{Ne} / \mathrm{O})$ & $-0.87 \pm 0.02$ & $-0.66 \pm 0.04$ & $-1.13 \pm 0.04$ & $-0.94 \pm 0.14$ \\
\hline $\mathrm{S}^{+} / \mathrm{H}^{+}\left(\times 10^{6}\right)$ & $0.11 \pm 0.00$ & $\ldots$ & $0.54 \pm 0.02$ & $0.83 \pm 0.07$ \\
\hline $\mathrm{S}^{++} / \mathrm{H}^{+}\left(\times 10^{6}\right)$ & $0.89 \pm 0.04$ & $\ldots$ & $1.09 \pm 0.07$ & $3.31 \pm 0.53$ \\
\hline ICF & $2.30 \pm 0.08$ & $\ldots$ & $1.02 \pm 0.02$ & $1.06 \pm 0.08$ \\
\hline $\log (\mathrm{S} / \mathrm{O})$ & $-1.52 \pm 0.02$ & $\cdots$ & $-1.65 \pm 0.03$ & $-1.76 \pm 0.09$ \\
\hline $\mathrm{Cl}^{++} / \mathrm{H}^{+}\left(\times 10^{8}\right)$ & $1.41 \pm 0.10$ & $\ldots$ & $3.76 \pm 0.34$ & $8.07 \pm 1.30$ \\
\hline ICF & $2.75 \pm 0.78$ & $\ldots$ & $1.31 \pm 0.14$ & $1.14 \pm 0.12$ \\
\hline $\log (\mathrm{Cl} / \mathrm{O})$ & $-3.29 \pm 0.13$ & $\ldots$ & $-3.18 \pm 0.06$ & $-3.44 \pm 0.10$ \\
\hline $\mathrm{Ar}^{++} / \mathrm{H}^{+}\left(\times 10^{7}\right)$ & $\ldots$ & $\ldots$ & $5.60 \pm 0.21$ & $7.38 \pm 0.72$ \\
\hline $\mathrm{Ar}^{+++} / \mathrm{H}^{+}\left(\times 10^{7}\right)$ & $\ldots$ & $\ldots$ & $\ldots$ & $\ldots$ \\
\hline ICF & $\ldots$ & $\ldots$ & $1.10 \pm 0.02$ & $1.08 \pm 0.05$ \\
\hline $\log (\mathrm{Ar} / \mathrm{O})$ & $\ldots$ & $\ldots$ & $-2.08 \pm 0.03$ & $-2.50 \pm 0.07$ \\
\hline $\mathrm{Fe}^{++} / \mathrm{H}^{+}\left(\times 10^{6}\right)(4658)$ & $\ldots$ & $0.42 \pm 0.03$ & $0.54 \pm 0.03$ & $1.34 \pm 0.17$ \\
\hline & $\ldots$ & $5.00 \pm 0.28$ & $2.29 \pm 0.18$ & $3.63 \pm 0.55$ \\
\hline $\log (\mathrm{Fe} / \mathrm{O})(\lambda 4658)$ & $\ldots$ & $-1.88 \pm 0.04$ & $-1.78 \pm 0.05$ & $-1.72 \pm 0.10$ \\
\hline \multirow{3}{*}{ Property } & \multicolumn{4}{|c|}{$\begin{array}{c}\text { GalaXY } \\
\text { (ESO PROGRAM) }\end{array}$} \\
\hline & NGC 3125 & MRK 1259 & MRK 1271 & POX 4 \\
\hline & (081.C-0113(A)) & $(073 . \mathrm{B}-0283(\mathrm{~A}))$ & (081.C-0113(A)) & (081.C-0113(A)) \\
\hline$T_{\mathrm{e}}\left(\mathrm{O}_{\text {III }}\right)(\mathrm{K})$ & $11087 \pm 71$ & $10126 \pm 340$ & $13428 \pm 102$ & $13089 \pm 97$ \\
\hline$T_{\mathrm{e}}\left(\mathrm{O}_{\text {II }}\right)(\mathrm{K})$ & $11236 \pm 79$ & $10082 \pm 373$ & $12955 \pm 117$ & $12711 \pm 110$ \\
\hline$T_{\mathrm{e}}(\mathrm{S}$ III $)(\mathrm{K})$ & $11191 \pm 59$ & $10092 \pm 283$ & $12912 \pm 85$ & $12506 \pm 80$ \\
\hline$N_{\mathrm{e}}(\mathrm{S}$ II $)\left(\mathrm{cm}^{-3}\right)$ & $276 \pm 48$ & $1333 \pm 130$ & $87 \pm 36$ & $151 \pm 39$ \\
\hline $\mathrm{O}^{+} / \mathrm{H}^{+}\left(\times 10^{4}\right)$ & $0.24 \pm 0.01$ & $0.74 \pm 0.11$ & $0.15 \pm 0.00$ & $0.13 \pm 0.00$ \\
\hline $\mathrm{O}^{++} / \mathrm{H}^{+}\left(\times 10^{4}\right)$ & $1.51 \pm 0.03$ & $0.57 \pm 0.06$ & $0.82 \pm 0.02$ & $1.03 \pm 0.02$ \\
\hline $\mathrm{O}^{+++} / \mathrm{H}^{+}\left(\times 10^{6}\right)$ & $6.45 \pm 0.29$ & $\ldots$ & $0.20 \pm 0.01$ & $1.02 \pm 0.04$ \\
\hline $\mathrm{O} / \mathrm{H}\left(\times 10^{4}\right)$ & $1.81 \pm 0.04$ & $1.31 \pm 0.12$ & $0.98 \pm 0.02$ & $1.17 \pm 0.02$ \\
\hline $12+\log (\mathrm{O} / \mathrm{H})$ & $8.26 \pm 0.01$ & $8.12 \pm 0.04$ & $7.99 \pm 0.01$ & $8.07 \pm 0.01$ \\
\hline $\mathrm{N}^{+} / \mathrm{H}^{+}\left(\times 10^{6}\right)$ & $1.48 \pm 0.05$ & $15.67 \pm 2.42$ & $0.47 \pm 0.02$ & $0.36 \pm 0.01$ \\
\hline ICF & $6.80 \pm 0.18$ & $2.04 \pm 0.26$ & $5.91 \pm 0.18$ & $7.99 \pm 0.25$ \\
\hline $\log (\mathrm{N} / \mathrm{O})$ & $-1.25 \pm 0.02$ & $-0.61 \pm 0.10$ & $-1.55 \pm 0.02$ & $-1.61 \pm 0.02$ \\
\hline $\mathrm{Ne}^{++} / \mathrm{H}^{+}\left(\times 10^{5}\right)$ & $4.08 \pm 0.11$ & $0.70 \pm 0.09$ & $1.97 \pm 0.05$ & $1.82 \pm 0.05$ \\
\hline ICF & $1.09 \pm 0.02$ & $1.78 \pm 0.17$ & $1.07 \pm 0.02$ & $1.03 \pm 0.02$ \\
\hline $\log (\mathrm{Ne} / \mathrm{O})$ & $-0.61 \pm 0.02$ & $-1.03 \pm 0.08$ & $-0.66 \pm 0.02$ & $-0.79 \pm 0.02$ \\
\hline $\mathrm{S}^{+} / \mathrm{H}^{+}\left(\times 10^{6}\right)$ & $0.38 \pm 0.01$ & $0.84 \pm 0.07$ & $0.20 \pm 0.00$ & $0.18 \pm 0.00$ \\
\hline $\mathrm{S}^{++} / \mathrm{H}^{+}\left(\times 10^{6}\right)$ & $2.94 \pm 0.08$ & $1.70 \pm 0.19$ & $1.07 \pm 0.03$ & $1.54 \pm 0.04$ \\
\hline ICF & $1.80 \pm 0.04$ & $1.00 \pm 0.05$ & $1.43 \pm 0.03$ & $1.81 \pm 0.04$ \\
\hline
\end{tabular}


Table 6. continued.

\begin{tabular}{|c|c|c|c|c|}
\hline \multirow[b]{2}{*}{ Property } & \multicolumn{4}{|c|}{$\begin{array}{c}\text { GALAXY } \\
\text { (ESO PROGRAM) }\end{array}$} \\
\hline & $\begin{array}{c}\text { NGC 3125 } \\
(081 . C-0113(\mathrm{~A}))\end{array}$ & $\begin{array}{c}\text { MRK 1259 } \\
(073 . \mathrm{B}-0283(\mathrm{~A}))\end{array}$ & $\begin{array}{c}\text { MRK 1271 } \\
(081 . C-0113(\mathrm{~A}))\end{array}$ & $\begin{array}{c}\text { POX 4 } \\
(081 . C-0113(\mathrm{~A}))\end{array}$ \\
\hline $\log (\mathrm{S} / \mathrm{O})$ & $-1.48 \pm 0.02$ & $-1.72 \pm 0.06$ & $-1.73 \pm 0.02$ & $-1.58 \pm 0.02$ \\
\hline $\begin{array}{l}\mathrm{Cl}^{++} / \mathrm{H}^{+}\left(\times 10^{8}\right) \\
\mathrm{ICF} \\
\log (\mathrm{Cl} / \mathrm{O})\end{array}$ & $\begin{array}{r}3.37 \pm 0.12 \\
1.43 \pm 0.03 \\
-3.57 \pm 0.02\end{array}$ & $\begin{array}{l}\cdots \\
\cdots \\
\cdots\end{array}$ & $\begin{array}{r}1.42 \pm 0.06 \\
1.45 \pm 0.19 \\
-3.68 \pm 0.06\end{array}$ & $\begin{array}{r}2.14 \pm 0.08 \\
1.69 \pm 0.23 \\
-3.51 \pm 0.06\end{array}$ \\
\hline $\begin{array}{l}\mathrm{Ar}^{++} / \mathrm{H}^{+}\left(\times 10^{7}\right) \\
\mathrm{Ar}^{+++} / \mathrm{H}^{+}\left(\times 10^{7}\right) \\
\mathrm{ICF} \\
\log (\mathrm{Ar} / \mathrm{O})\end{array}$ & $\begin{array}{r}9.25 \pm 0.19 \\
1.09 \pm 0.04 \\
1.15 \pm 0.01 \\
-2.23 \pm 0.01\end{array}$ & $\begin{array}{c}5.35 \pm 0.38 \\
\ldots \\
1.12 \pm 0.05 \\
-2.34 \pm 0.05\end{array}$ & $\begin{array}{r}3.23 \pm 0.07 \\
0.96 \pm 0.02 \\
1.16 \pm 0.01 \\
-2.42 \pm 0.01\end{array}$ & $\begin{array}{r}3.02 \pm 0.06 \\
1.37 \pm 0.03 \\
1.24 \pm 0.02 \\
-2.49 \pm 0.01\end{array}$ \\
\hline $\begin{array}{l}\mathrm{Fe}^{++} / \mathrm{H}^{+}\left(\times 10^{6}\right)(4658) \\
\mathrm{ICF} \\
\log (\mathrm{Fe} / \mathrm{O})(\lambda 4658)\end{array}$ & $\begin{array}{r}0.24 \pm 0.01 \\
9.39 \pm 0.28 \\
-1.91 \pm 0.02 \\
\end{array}$ & $\begin{array}{l}\cdots \\
\cdots \\
\cdots\end{array}$ & $\begin{array}{r}0.13 \pm 0.00 \\
8.20 \pm 0.29 \\
-1.97 \pm 0.02\end{array}$ & $\begin{array}{r}0.14 \pm 0.00 \\
11.34 \pm 0.39 \\
-1.87 \pm 0.02 \\
\end{array}$ \\
\hline \multirow[b]{2}{*}{ Property } & \multicolumn{4}{|c|}{$\begin{array}{c}\text { GALAXY } \\
\text { (ESO PROGRAM) }\end{array}$} \\
\hline & $\begin{array}{l}\text { TOL 1214-277 } \\
(69 . \mathrm{D}-0174(\mathrm{~A}))\end{array}$ & $\begin{array}{c}\mathrm{J} 1253-0312 \\
(081 . \mathrm{C}-0113(\mathrm{~A}))\end{array}$ & $\begin{array}{l}\text { NGC 5253 No.C1 } \\
(073 . \mathrm{B}-0283(\mathrm{~A}))\end{array}$ & $\begin{array}{c}\text { NGC } 5253 \text { No.C2 } \\
(073 . \mathrm{B}-0283(\mathrm{~A}))\end{array}$ \\
\hline $\begin{array}{l}T_{\mathrm{e}}(\mathrm{O} \text { III })(\mathrm{K}) \\
T_{\mathrm{e}}(\mathrm{O} \text { II })(\mathrm{K}) \\
T_{\mathrm{e}}(\mathrm{S} \text { III })(\mathrm{K}) \\
N_{\mathrm{e}}(\mathrm{S} \text { II })\left(\mathrm{cm}^{-3}\right)\end{array}$ & $\begin{array}{r}20020 \pm 226 \\
15146 \pm 287 \\
18458 \pm 188 \\
10 \pm 10\end{array}$ & $\begin{array}{r}13851 \pm 108 \\
13241 \pm 124 \\
13401 \pm 90 \\
1726 \pm 153\end{array}$ & $\begin{array}{r}12279 \pm 85 \\
12071 \pm 96 \\
11485 \pm 71 \\
723 \pm 69\end{array}$ & $\begin{array}{r}10267 \pm 65 \\
10219 \pm 72 \\
10235 \pm 54 \\
271 \pm 47\end{array}$ \\
\hline $\begin{array}{l}\mathrm{O}^{+} / \mathrm{H}^{+}\left(\times 10^{4}\right) \\
\mathrm{O}^{++} / \mathrm{H}^{+}\left(\times 10^{4}\right) \\
\mathrm{O}^{+++} / \mathrm{H}^{+}\left(\times 10^{6}\right) \\
\mathrm{O} / \mathrm{H}\left(\times 10^{4}\right) \\
12+\log (\mathrm{O} / \mathrm{H})\end{array}$ & $\begin{array}{l}0.03 \pm 0.00 \\
0.29 \pm 0.01 \\
1.70 \pm 0.06 \\
0.34 \pm 0.01 \\
7.53 \pm 0.01\end{array}$ & $\begin{array}{l}0.13 \pm 0.00 \\
0.87 \pm 0.02 \\
0.64 \pm 0.02 \\
1.01 \pm 0.02 \\
8.00 \pm 0.01\end{array}$ & $\begin{array}{c}0.20 \pm 0.01 \\
1.20 \pm 0.03 \\
\ldots \\
1.39 \pm 0.03 \\
8.14 \pm 0.01\end{array}$ & $\begin{array}{c}0.56 \pm 0.02 \\
1.49 \pm 0.04 \\
\ldots \\
2.05 \pm 0.04 \\
8.31 \pm 0.01\end{array}$ \\
\hline $\begin{array}{l}\mathrm{N}^{+} / \mathrm{H}^{+}\left(\times 10^{6}\right) \\
\mathrm{ICF} \\
\log (\mathrm{N} / \mathrm{O})\end{array}$ & $\begin{array}{r}0.06 \pm 0.00 \\
11.22 \pm 0.60 \\
-1.70 \pm 0.04\end{array}$ & $\begin{array}{r}1.27 \pm 0.05 \\
6.88 \pm 0.22 \\
-1.06 \pm 0.02\end{array}$ & $\begin{array}{r}2.52 \pm 0.09 \\
6.48 \pm 0.19 \\
-0.93 \pm 0.02\end{array}$ & $\begin{array}{r}2.22 \pm 0.07 \\
3.87 \pm 0.09 \\
-1.38 \pm 0.02\end{array}$ \\
\hline $\begin{array}{l}\mathrm{Ne}^{++} / \mathrm{H}^{+}\left(\times 10^{5}\right) \\
\mathrm{ICF} \\
\log (\mathrm{Ne} / \mathrm{O})\end{array}$ & $\begin{array}{r}0.52 \pm 0.02 \\
1.05 \pm 0.01 \\
-0.79 \pm 0.02\end{array}$ & $\begin{array}{r}1.61 \pm 0.04 \\
1.05 \pm 0.02 \\
-0.78 \pm 0.02\end{array}$ & $\begin{array}{r}2.80 \pm 0.07 \\
1.05 \pm 0.02 \\
-0.67 \pm 0.02\end{array}$ & $\begin{array}{r}2.59 \pm 0.07 \\
1.25 \pm 0.03 \\
-0.80 \pm 0.02\end{array}$ \\
\hline $\begin{array}{l}\mathrm{S}^{+} / \mathrm{H}^{+}\left(\times 10^{6}\right) \\
\mathrm{S}^{++} / \mathrm{H}^{+}\left(\times 10^{6}\right) \\
\mathrm{ICF} \\
\log (\mathrm{S} / \mathrm{O})\end{array}$ & $\begin{array}{r}0.03 \pm 0.00 \\
0.24 \pm 0.01 \\
1.86 \pm 0.07 \\
-1.83 \pm 0.02\end{array}$ & $\begin{array}{r}0.16 \pm 0.00 \\
1.10 \pm 0.03 \\
1.57 \pm 0.03 \\
-1.71 \pm 0.02\end{array}$ & $\begin{array}{r}0.24 \pm 0.00 \\
3.00 \pm 0.08 \\
1.66 \pm 0.04 \\
-1.41 \pm 0.02\end{array}$ & $\begin{array}{r}0.59 \pm 0.01 \\
4.08 \pm 0.11 \\
1.22 \pm 0.02 \\
-1.55 \pm 0.02\end{array}$ \\
\hline $\begin{array}{l}\mathrm{Cl}^{++} / \mathrm{H}^{+}\left(\times 10^{8}\right) \\
\mathrm{ICF} \\
\log (\mathrm{Cl} / \mathrm{O})\end{array}$ & $\begin{array}{l}\cdots \\
\cdots \\
\cdots\end{array}$ & $\begin{array}{r}1.58 \pm 0.05 \\
1.57 \pm 0.23 \\
-3.61 \pm 0.07\end{array}$ & $\begin{array}{r}3.08 \pm 0.09 \\
1.43 \pm 0.12 \\
-3.50 \pm 0.04\end{array}$ & $\begin{array}{r}4.22 \pm 0.17 \\
1.12 \pm 0.02 \\
-3.63 \pm 0.02\end{array}$ \\
\hline $\begin{array}{l}\mathrm{Ar}^{++} / \mathrm{H}^{+}\left(\times 10^{7}\right) \\
\mathrm{Ar}^{+++} / \mathrm{H}^{+}\left(\times 10^{7}\right) \\
\mathrm{ICF} \\
\log (\mathrm{Ar} / \mathrm{O})\end{array}$ & $\begin{array}{r}0.59 \pm 0.01 \\
1.06 \pm 0.03 \\
1.49 \pm 0.04 \\
-2.58 \pm 0.03\end{array}$ & $\begin{array}{r}3.36 \pm 0.07 \\
1.24 \pm 0.03 \\
1.20 \pm 0.01 \\
-2.40 \pm 0.01\end{array}$ & $\begin{array}{r}8.59 \pm 0.18 \\
2.26 \pm 0.05 \\
1.15 \pm 0.01 \\
-2.15 \pm 0.01\end{array}$ & $\begin{array}{r}9.82 \pm 0.20 \\
0.58 \pm 0.04 \\
1.06 \pm 0.01 \\
-2.29 \pm 0.01\end{array}$ \\
\hline $\begin{array}{l}\mathrm{Fe}^{++} / \mathrm{H}^{+}\left(\times 10^{6}\right)(4658) \\
\mathrm{ICF} \\
\log (\mathrm{Fe} / \mathrm{O})(\lambda 4658)\end{array}$ & $\begin{array}{r}0.05 \pm 0.01 \\
16.81 \pm 0.97 \\
-1.63 \pm 0.08 \\
\end{array}$ & $\begin{array}{r}0.24 \pm 0.01 \\
9.68 \pm 0.35 \\
-1.64 \pm 0.02 \\
\end{array}$ & $\begin{array}{r}0.28 \pm 0.01 \\
8.97 \pm 0.29 \\
-1.74 \pm 0.02 \\
\end{array}$ & $\begin{array}{r}0.46 \pm 0.01 \\
5.07 \pm 0.14 \\
-1.95 \pm 0.02 \\
\end{array}$ \\
\hline \multirow[b]{2}{*}{ Property } & \multicolumn{4}{|c|}{$\begin{array}{c}\text { GALAXY } \\
\text { (ESO PROGRAM) }\end{array}$} \\
\hline & $\begin{array}{l}\text { NGC } 5253 \text { No.P1 } \\
(70 . C-0008(A))\end{array}$ & $\begin{array}{c}\text { NGC } 5253 \text { No.P2 } \\
(70 . C-0008(A))\end{array}$ & $\begin{array}{c}\text { TOL } 89 \text { No.1 } \\
(073 . \mathrm{B}-0283(\mathrm{~A}))\end{array}$ & $\begin{array}{c}\text { TOL } 89 \text { No. } 2 \\
(073 . \mathrm{B}-0283(\mathrm{~A}))\end{array}$ \\
\hline$T_{\mathrm{e}}\left(\mathrm{O}_{\text {IIII }}\right)(\mathrm{K})$ & $13531 \pm 56$ & $12412 \pm 87$ & $10263 \pm 74$ & $9377 \pm 160$ \\
\hline$T_{\mathrm{e}}(\mathrm{O}$ II) $(\mathrm{K})$ & $13027 \pm 65$ & $12182 \pm 99$ & $10215 \pm 81$ & $9546 \pm 174$ \\
\hline$T_{\mathrm{e}}(\mathrm{S}$ III $)(\mathrm{K})$ & $13027 \pm 47$ & $11659 \pm 72$ & $10231 \pm 61$ & $9441 \pm 133$ \\
\hline$N_{\mathrm{e}}(\mathrm{S}$ II $)\left(\mathrm{cm}^{-3}\right)$ & $366 \pm 50$ & $1108 \pm 99$ & $147 \pm 38$ & $149 \pm 52$ \\
\hline
\end{tabular}


Table 6. continued.

\begin{tabular}{|c|c|c|c|c|}
\hline \multirow[b]{2}{*}{ Property } & \multicolumn{4}{|c|}{$\begin{array}{c}\text { GALAXY } \\
\text { (ESO PROGRAM) }\end{array}$} \\
\hline & $\begin{array}{c}\text { NGC } 5253 \text { No.P1 } \\
(70 . C-0008(A))\end{array}$ & $\begin{array}{c}\text { NGC } 5253 \text { No.P2 } \\
(70 . C-0008(A))\end{array}$ & $\begin{array}{c}\text { TOL 89 No.1 } \\
(073 . \mathrm{B}-0283(\mathrm{~A}))\end{array}$ & $\begin{array}{c}\text { TOL 89 No.2 } \\
(073 . \mathrm{B}-0283(\mathrm{~A}))\end{array}$ \\
\hline $\mathrm{O}^{+} / \mathrm{H}^{+}\left(\times 10^{4}\right)$ & $0.26 \pm 0.01$ & $0.22 \pm 0.01$ & $0.56 \pm 0.02$ & $0.78 \pm 0.06$ \\
\hline $\mathrm{O}^{++} / \mathrm{H}^{+}\left(\times 10^{4}\right)$ & $0.73 \pm 0.01$ & $1.19 \pm 0.03$ & $1.63 \pm 0.04$ & $1.75 \pm 0.11$ \\
\hline $\mathrm{O} / \mathrm{H}\left(\times 10^{4}\right)$ & $0.99 \pm 0.01$ & $1.41 \pm 0.03$ & $2.19 \pm 0.05$ & $2.53 \pm 0.12$ \\
\hline $12+\log (\mathrm{O} / \mathrm{H})$ & $8.00 \pm 0.01$ & $8.15 \pm 0.01$ & $8.34 \pm 0.01$ & $8.40 \pm 0.02$ \\
\hline $\mathrm{N}^{+} / \mathrm{H}^{+}\left(\times 10^{6}\right)$ & $0.94 \pm 0.02$ & $3.20 \pm 0.11$ & $2.35 \pm 0.08$ & $2.93 \pm 0.22$ \\
\hline ICF & $3.84 \pm 0.07$ & $6.03 \pm 0.17$ & $4.05 \pm 0.11$ & $3.51 \pm 0.20$ \\
\hline $\log (\mathrm{N} / \mathrm{O})$ & $-1.44 \pm 0.01$ & $-0.86 \pm 0.02$ & $-1.36 \pm 0.02$ & $-1.39 \pm 0.05$ \\
\hline $\mathrm{Ne}^{++} / \mathrm{H}^{+}\left(\times 10^{5}\right)$ & $1.49 \pm 0.03$ & $2.97 \pm 0.08$ & $3.34 \pm 0.10$ & $2.58 \pm 0.18$ \\
\hline ICF & $1.17 \pm 0.01$ & $1.07 \pm 0.02$ & $1.22 \pm 0.03$ & $1.31 \pm 0.07$ \\
\hline $\log (\mathrm{Ne} / \mathrm{O})$ & $-0.75 \pm 0.01$ & $-0.65 \pm 0.02$ & $-0.73 \pm 0.02$ & $-0.87 \pm 0.04$ \\
\hline $\mathrm{S}^{+} / \mathrm{H}^{+}\left(\times 10^{6}\right)$ & $0.25 \pm 0.00$ & $0.24 \pm 0.00$ & $0.45 \pm 0.01$ & $1.10 \pm 0.05$ \\
\hline $\mathrm{S}^{++} / \mathrm{H}^{+}\left(\times 10^{6}\right)$ & $1.51 \pm 0.04$ & $3.09 \pm 0.09$ & $3.48 \pm 0.10$ & $4.08 \pm 0.33$ \\
\hline ICF & $1.18 \pm 0.01$ & $1.58 \pm 0.03$ & $1.25 \pm 0.02$ & $1.06 \pm 0.02$ \\
\hline $\log (\mathrm{S} / \mathrm{O})$ & $-1.68 \pm 0.01$ & $-1.43 \pm 0.02$ & $-1.65 \pm 0.02$ & $-1.63 \pm 0.04$ \\
\hline $\mathrm{Cl}^{++} / \mathrm{H}^{+}\left(\times 10^{8}\right)$ & $2.52 \pm 0.18$ & $2.92 \pm 0.14$ & $4.89 \pm 0.17$ & $6.17 \pm 0.63$ \\
\hline $\mathrm{ICF}$ & $1.23 \pm 0.08$ & $1.37 \pm 0.11$ & $1.13 \pm 0.02$ & $1.12 \pm 0.05$ \\
\hline $\log (\mathrm{Cl} / \mathrm{O})$ & $-3.51 \pm 0.04$ & $-3.55 \pm 0.04$ & $-3.60 \pm 0.02$ & $-3.57 \pm 0.05$ \\
\hline $\mathrm{Ar}^{++} / \mathrm{H}^{+}\left(\times 10^{7}\right)$ & $5.67 \pm 0.10$ & $8.51 \pm 0.18$ & $9.23 \pm 0.20$ & $22.95 \pm 0.93$ \\
\hline $\mathrm{Ar}^{+++} / \mathrm{H}^{+}\left(\times 10^{7}\right)$ & $0.18 \pm 0.03$ & $1.66 \pm 0.06$ & $0.64 \pm 0.04$ & $\ldots$ \\
\hline ICF & $1.08 \pm 0.01$ & $1.13 \pm 0.01$ & $1.06 \pm 0.01$ & $1.06 \pm 0.02$ \\
\hline $\log (\mathrm{Ar} / \mathrm{O})$ & $-2.21 \pm 0.01$ & $-2.17 \pm 0.01$ & $-2.35 \pm 0.01$ & $-2.02 \pm 0.03$ \\
\hline $\mathrm{Fe}^{++} / \mathrm{H}^{+}\left(\times 10^{6}\right)(4658)$ & $0.19 \pm 0.01$ & $0.29 \pm 0.01$ & $0.29 \pm 0.01$ & $0.57 \pm 0.06$ \\
\hline ICF & $5.10 \pm 0.12$ & $8.28 \pm 0.26$ & $5.32 \pm 0.16$ & $4.55 \pm 0.31$ \\
\hline $\log (\mathrm{Fe} / \mathrm{O})(\lambda 4658)$ & $-2.02 \pm 0.02$ & $-1.76 \pm 0.02$ & $-2.15 \pm 0.02$ & $-1.99 \pm 0.06$ \\
\hline \multirow[b]{2}{*}{ Property } & \multicolumn{4}{|c|}{$\begin{array}{c}\text { GALAXY } \\
\text { (ESO PROGRAM) }\end{array}$} \\
\hline & $\begin{array}{l}\text { NGC 5408 No.1 } \\
(081 . C-0113(A))\end{array}$ & $\begin{array}{l}\text { NGC 5408 No.2 } \\
(081 . C-0113(\mathrm{~A}))\end{array}$ & $\begin{array}{c}\text { TOL 1457-262 } \\
(081 \mathrm{C}-0113(\mathrm{~A}))\end{array}$ & $\begin{array}{c}\text { TOL 1924-416 No.1 } \\
(081 \text { C- } 0113(\mathrm{~A}))\end{array}$ \\
\hline$T_{\mathrm{e}}(\mathrm{O}$ III $)(\mathrm{K})$ & $15585 \pm 132$ & $13667 \pm 107$ & $11892 \pm 81$ & $12485 \pm 96$ \\
\hline$T_{\mathrm{e}}(\mathrm{O}$ II $)(\mathrm{K})$ & $14181 \pm 155$ & $13119 \pm 123$ & $11737 \pm 91$ & $12242 \pm 108$ \\
\hline$T_{\mathrm{e}}\left(\mathrm{S}_{\text {IIII }}(\mathrm{K})\right.$ & $15200 \pm 109$ & $13190 \pm 89$ & $10973 \pm 68$ & $11752 \pm 79$ \\
\hline$N_{\mathrm{e}}(\mathrm{S}$ II $)\left(\mathrm{cm}^{-3}\right)$ & $308 \pm 46$ & $133 \pm 36$ & $104 \pm 35$ & $126 \pm 37$ \\
\hline $\mathrm{O}^{+} / \mathrm{H}^{+}\left(\times 10^{4}\right)$ & $0.13 \pm 0.00$ & $0.28 \pm 0.01$ & $0.55 \pm 0.02$ & $0.17 \pm 0.01$ \\
\hline $\mathrm{O}^{++} / \mathrm{H}^{+}\left(\times 10^{4}\right)$ & $0.94 \pm 0.02$ & $1.07 \pm 0.03$ & $1.31 \pm 0.03$ & $0.78 \pm 0.02$ \\
\hline $\mathrm{O}^{+++} / \mathrm{H}^{+}\left(\times 10^{6}\right)$ & $0.91 \pm 0.03$ & $0.60 \pm 0.03$ & $1.07 \pm 0.05$ & $2.56 \pm 0.12$ \\
\hline $\mathrm{O} / \mathrm{H}\left(\times 10^{4}\right)$ & $1.08 \pm 0.02$ & $1.36 \pm 0.03$ & $1.87 \pm 0.03$ & $0.98 \pm 0.02$ \\
\hline $12+\log (\mathrm{O} / \mathrm{H})$ & $8.03 \pm 0.01$ & $8.13 \pm 0.01$ & $8.27 \pm 0.01$ & $7.99 \pm 0.01$ \\
\hline $\mathrm{N}^{+} / \mathrm{H}^{+}\left(\times 10^{6}\right)$ & $0.22 \pm 0.01$ & $0.51 \pm 0.02$ & $1.02 \pm 0.03$ & $0.82 \pm 0.03$ \\
\hline ICF & $7.44 \pm 0.25$ & $4.77 \pm 0.13$ & $3.66 \pm 0.08$ & $5.44 \pm 0.17$ \\
\hline $\log (\mathrm{N} / \mathrm{O})$ & $-1.81 \pm 0.02$ & $-1.75 \pm 0.02$ & $-1.70 \pm 0.02$ & $-1.34 \pm 0.02$ \\
\hline $\mathrm{Ne}^{++} / \mathrm{H}^{+}\left(\times 10^{5}\right)$ & $2.06 \pm 0.05$ & $2.16 \pm 0.06$ & $2.85 \pm 0.08$ & $1.46 \pm 0.04$ \\
\hline ICF & $1.05 \pm 0.02$ & $1.14 \pm 0.02$ & $1.29 \pm 0.03$ & $1.11 \pm 0.02$ \\
\hline $\log (\mathrm{Ne} / \mathrm{O})$ & $-0.70 \pm 0.02$ & $-0.74 \pm 0.02$ & $-0.71 \pm 0.02$ & $-0.78 \pm 0.02$ \\
\hline $\mathrm{S}^{+} / \mathrm{H}^{+}\left(\times 10^{6}\right)$ & $0.13 \pm 0.00$ & $0.34 \pm 0.01$ & $0.33 \pm 0.01$ & $0.24 \pm 0.01$ \\
\hline $\mathrm{S}^{++} / \mathrm{H}^{+}\left(\times 10^{6}\right)$ & $0.99 \pm 0.02$ & $2.13 \pm 0.06$ & $2.34 \pm 0.07$ & $1.57 \pm 0.05$ \\
\hline ICF & $1.68 \pm 0.04$ & $1.35 \pm 0.03$ & $1.18 \pm 0.02$ & $1.13 \pm 0.01$ \\
\hline $\log (\mathrm{S} / \mathrm{O})$ & $-1.76 \pm 0.02$ & $-1.61 \pm 0.02$ & $-1.77 \pm 0.02$ & $-1.60 \pm 0.02$ \\
\hline $\mathrm{Cl}^{++} / \mathrm{H}^{+}\left(\times 10^{8}\right)$ & $1.64 \pm 0.10$ & $3.27 \pm 0.18$ & $3.79 \pm 0.27$ & $2.17 \pm 0.13$ \\
\hline ICF & $1.63 \pm 0.25$ & $1.23 \pm 0.09$ & $1.12 \pm 0.02$ & $1.39 \pm 0.18$ \\
\hline $\log (\mathrm{Cl} / \mathrm{O})$ & $-3.61 \pm 0.07$ & $-3.53 \pm 0.04$ & $-3.65 \pm 0.03$ & $-3.51 \pm 0.06$ \\
\hline
\end{tabular}


Table 6. continued.

\begin{tabular}{|c|c|c|c|c|}
\hline \multirow[b]{2}{*}{ Property } & \multicolumn{4}{|c|}{$\begin{array}{c}\text { GALAXY } \\
\text { (ESO PROGRAM) }\end{array}$} \\
\hline & $\begin{array}{l}\text { NGC 5408 No.1 } \\
(081 . C-0113(\mathrm{~A}))\end{array}$ & $\begin{array}{l}\text { NGC 5408 No.2 } \\
(081 . C-0113(\mathrm{~A})) \\
\end{array}$ & $\begin{array}{c}\text { TOL 1457-262 } \\
(081 . \mathrm{C}-0113(\mathrm{~A})) \\
\end{array}$ & $\begin{array}{l}\text { TOL 1924-416 No.1 } \\
(081 . \mathrm{C}-0113(\mathrm{~A})) \\
\end{array}$ \\
\hline $\mathrm{Ar}^{++} / \mathrm{H}^{+}\left(\times 10^{7}\right)$ & $2.84 \pm 0.06$ & $4.77 \pm 0.10$ & $5.00 \pm 0.11$ & $3.04 \pm 0.07$ \\
\hline $\mathrm{Ar}^{+++} / \mathrm{H}^{+}\left(\times 10^{7}\right)$ & $\ldots$ & $\ldots$ & $0.83 \pm 0.04$ & $0.48 \pm 0.02$ \\
\hline ICF & $1.22 \pm 0.02$ & $1.09 \pm 0.01$ & $1.06 \pm 0.01$ & $1.13 \pm 0.01$ \\
\hline $\log (\mathrm{Ar} / \mathrm{O})$ & $-2.49 \pm 0.01$ & $-2.42 \pm 0.01$ & $-2.55 \pm 0.01$ & $-2.45 \pm 0.01$ \\
\hline $\mathrm{Fe}^{++} / \mathrm{H}^{+}\left(\times 10^{6}\right)(4658)$ & $0.06 \pm 0.00$ & $0.06 \pm 0.00$ & $0.62 \pm 0.03$ & $0.26 \pm 0.01$ \\
\hline ICF & $10.52 \pm 0.39$ & $6.40 \pm 0.20$ & $4.77 \pm 0.13$ & $7.48 \pm 0.27$ \\
\hline $\log (\mathrm{Fe} / \mathrm{O})(\lambda 4658)$ & $-2.23 \pm 0.02$ & $-2.52 \pm 0.03$ & $-1.80 \pm 0.02$ & $-1.71 \pm 0.02$ \\
\hline \multirow[b]{2}{*}{ Property } & \multicolumn{3}{|c|}{$\begin{array}{c}\text { GALAXY } \\
\text { (ESO PROGRAM) }\end{array}$} & \\
\hline & $\begin{array}{l}\text { TOL 1924-416 No.2 } \\
(081 . \mathrm{C}-0113(\mathrm{~A}))\end{array}$ & $\begin{array}{c}\text { NGC 6822 V } \\
(081 . C-0113(\mathrm{~A}))\end{array}$ & $\begin{array}{c}\text { PHL 293B } \\
(70 . \mathrm{B}-0717(\mathrm{~A}))\end{array}$ & \\
\hline$T_{\mathrm{e}}(\mathrm{O}$ III) $(\mathrm{K})$ & $12269 \pm 86$ & $11429 \pm 71$ & $16926 \pm 190$ & \\
\hline$T_{\mathrm{e}}\left(\mathrm{O}_{\text {II }}\right)(\mathrm{K})$ & $12063 \pm 97$ & $11712 \pm 79$ & $14657 \pm 230$ & \\
\hline$T_{\mathrm{e}}(\mathrm{S}$ III) $(\mathrm{K})$ & $11473 \pm 72$ & $11483 \pm 59$ & $16367 \pm 158$ & \\
\hline$N_{\mathrm{e}}(\mathrm{S}$ II $)\left(\mathrm{cm}^{-3}\right)$ & $178 \pm 41$ & $133 \pm 37$ & $41 \pm 41$ & \\
\hline $\mathrm{O}^{+} / \mathrm{H}^{+}\left(\times 10^{4}\right)$ & $0.22 \pm 0.01$ & $0.17 \pm 0.00$ & $0.05 \pm 0.00$ & \\
\hline $\mathrm{O}^{++} / \mathrm{H}^{+}\left(\times 10^{4}\right)$ & $1.00 \pm 0.02$ & $1.27 \pm 0.03$ & $0.48 \pm 0.01$ & \\
\hline $\mathrm{O}^{+++} / \mathrm{H}^{+}\left(\times 10^{6}\right)$ & $1.45 \pm 0.06$ & $\ldots$ & $\ldots$ & \\
\hline $\mathrm{O} / \mathrm{H}\left(\times 10^{4}\right)$ & $1.24 \pm 0.02$ & $1.43 \pm 0.03$ & $0.53 \pm 0.01$ & \\
\hline $12+\log (\mathrm{O} / \mathrm{H})$ & $8.09 \pm 0.01$ & $8.16 \pm 0.01$ & $7.72 \pm 0.01$ & \\
\hline $\mathrm{N}^{+} / \mathrm{H}^{+}\left(\times 10^{6}\right)$ & $0.65 \pm 0.02$ & $0.56 \pm 0.02$ & $0.14 \pm 0.01$ & \\
\hline ICF & $5.37 \pm 0.15$ & $7.49 \pm 0.20$ & $9.73 \pm 0.47$ & \\
\hline $\log (\mathrm{N} / \mathrm{O})$ & $-1.55 \pm 0.02$ & $-1.53 \pm 0.02$ & $-1.59 \pm 0.04$ & \\
\hline $\mathrm{Ne}^{++} / \mathrm{H}^{+}\left(\times 10^{5}\right)$ & $1.78 \pm 0.05$ & $2.32 \pm 0.06$ & $0.93 \pm 0.03$ & \\
\hline ICF & $1.11 \pm 0.02$ & $1.03 \pm 0.02$ & $1.03 \pm 0.02$ & \\
\hline $\log (\mathrm{Ne} / \mathrm{O})$ & $-0.80 \pm 0.02$ & $-0.78 \pm 0.02$ & $-0.74 \pm 0.02$ & \\
\hline $\mathrm{S}^{+} / \mathrm{H}^{+}\left(\times 10^{6}\right)$ & $0.29 \pm 0.01$ & $0.17 \pm 0.00$ & $0.09 \pm 0.00$ & \\
\hline $\mathrm{S}^{++} / \mathrm{H}^{+}\left(\times 10^{6}\right)$ & $2.22 \pm 0.06$ & $2.94 \pm 0.07$ & $0.64 \pm 0.02$ & \\
\hline ICF & $1.42 \pm 0.03$ & $1.87 \pm 0.04$ & $1.17 \pm 0.05$ & \\
\hline $\log (\mathrm{S} / \mathrm{O})$ & $-1.54 \pm 0.02$ & $-1.39 \pm 0.02$ & $-1.66 \pm 0.02$ & \\
\hline $\mathrm{Cl}^{++} / \mathrm{H}^{+}\left(\times 10^{8}\right)$ & $2.23 \pm 0.10$ & $3.51 \pm 0.09$ & $0.97 \pm 0.08$ & \\
\hline ICF & $1.32 \pm 0.13$ & $1.57 \pm 0.12$ & $2.07 \pm 0.60$ & \\
\hline $\log (\mathrm{Cl} / \mathrm{O})$ & $-3.62 \pm 0.05$ & $-3.42 \pm 0.04$ & $-3.42 \pm 0.13$ & \\
\hline $\mathrm{Ar}^{++} / \mathrm{H}^{+}\left(\times 10^{7}\right)$ & $4.17 \pm 0.09$ & $7.10 \pm 0.14$ & $\ldots$ & \\
\hline $\mathrm{Ar}^{+++} / \mathrm{H}^{+}\left(\times 10^{7}\right)$ & $0.77 \pm 0.04$ & $0.43 \pm 0.02$ & $\ldots$ & \\
\hline ICF & $1.11 \pm 0.01$ & $1.20 \pm 0.01$ & $\ldots$ & \\
\hline $\log (\mathrm{Ar} / \mathrm{O})$ & $-2.43 \pm 0.01$ & $-2.23 \pm 0.01$ & $\ldots$ & \\
\hline $\mathrm{Fe}^{++} / \mathrm{H}^{+}\left(\times 10^{6}\right)(4658)$ & $0.30 \pm 0.01$ & $0.07 \pm 0.00$ & $\ldots$ & \\
\hline ICF & $7.33 \pm 0.23$ & $10.48 \pm 0.31$ & $\ldots$ & \\
\hline $\log (\mathrm{Fe} / \mathrm{O})(\lambda 4658)$ & $-1.75 \pm 0.02$ & $-2.27 \pm 0.02$ & $\ldots$ & \\
\hline
\end{tabular}


N. G. Guseva et al.: Low-metallicity ELGs: deep VLT/FORS+UVES spectroscopy

Table 7. Ionic and total heavy element abundances (FORS low-resolution observations).

\begin{tabular}{|c|c|c|c|c|}
\hline \multirow[b]{2}{*}{ Property } & \multicolumn{4}{|c|}{$\begin{array}{c}\text { GALAXY } \\
\text { (ESO PROGRAM) }\end{array}$} \\
\hline & $\begin{array}{c}\text { NGC 346A No.1 } \\
(69 . C-0203(\mathrm{~A}))\end{array}$ & $\begin{array}{c}\text { NGC 346A No.2 } \\
(69 . C-0203(\mathrm{~A}))\end{array}$ & $\begin{array}{c}\text { NGC 346A No.3 } \\
(69 . C-0203(\mathrm{~A}))\end{array}$ & $\begin{array}{c}\text { NGC 346B No.1 } \\
(69 . C-0203(A))\end{array}$ \\
\hline$T_{\mathrm{e}}(\mathrm{O}$ III $)(\mathrm{K})$ & $12300 \pm 94$ & $12685 \pm 105$ & $12370 \pm 149$ & $12392 \pm 123$ \\
\hline$T_{\mathrm{e}}\left(\mathrm{O}_{\text {II }}(\mathrm{K})\right.$ & $12088 \pm 106$ & $12402 \pm 119$ & $12147 \pm 168$ & $12166 \pm 138$ \\
\hline$T_{\mathrm{e}}\left(\mathrm{S}_{\text {III }}\right)(\mathrm{K})$ & $11513 \pm 78$ & $12007 \pm 87$ & $11604 \pm 124$ & $11633 \pm 102$ \\
\hline$N_{\mathrm{e}}(\mathrm{S}$ II $)\left(\mathrm{cm}^{-3}\right)$ & $10 \pm 10$ & $52 \pm 31$ & $77 \pm 36$ & $36 \pm 29$ \\
\hline $\mathrm{O}^{+} / \mathrm{H}^{+}\left(\times 10^{4}\right)$ & $0.14 \pm 0.00$ & $0.16 \pm 0.01$ & $0.20 \pm 0.01$ & $0.25 \pm 0.01$ \\
\hline $\mathrm{O}^{++} / \mathrm{H}^{+}\left(\times 10^{4}\right)$ & $1.04 \pm 0.03$ & $0.91 \pm 0.02$ & $0.91 \pm 0.03$ & $0.89 \pm 0.03$ \\
\hline $\mathrm{O} / \mathrm{H}\left(\times 10^{4}\right)$ & $1.18 \pm 0.03$ & $1.07 \pm 0.02$ & $1.11 \pm 0.04$ & $1.13 \pm 0.03$ \\
\hline $12+\log (\mathrm{O} / \mathrm{H})$ & $8.07 \pm 0.01$ & $8.03 \pm 0.01$ & $8.04 \pm 0.01$ & $8.05 \pm 0.01$ \\
\hline $\mathrm{N}^{+} / \mathrm{H}^{+}\left(\times 10^{6}\right)$ & $0.24 \pm 0.01$ & $0.42 \pm 0.02$ & $0.47 \pm 0.03$ & $0.57 \pm 0.03$ \\
\hline ICF & $7.42 \pm 0.25$ & $6.16 \pm 0.21$ & $5.38 \pm 0.25$ & $4.53 \pm 0.17$ \\
\hline $\log (\mathrm{N} / \mathrm{O})$ & $-1.82 \pm 0.02$ & $-1.62 \pm 0.03$ & $-1.64 \pm 0.03$ & $-1.64 \pm 0.03$ \\
\hline $\mathrm{Ne}^{++} / \mathrm{H}^{+}\left(\times 10^{5}\right)$ & $1.85 \pm 0.05$ & $1.86 \pm 0.06$ & $1.89 \pm 0.08$ & $1.74 \pm 0.06$ \\
\hline ICF & $1.04 \pm 0.02$ & $1.07 \pm 0.02$ & $1.09 \pm 0.03$ & $1.14 \pm 0.03$ \\
\hline $\log (\mathrm{Ne} / \mathrm{O})$ & $-0.79 \pm 0.02$ & $-0.73 \pm 0.02$ & $-0.73 \pm 0.03$ & $-0.76 \pm 0.02$ \\
\hline $\mathrm{S}^{+} / \mathrm{H}^{+}\left(\times 10^{6}\right)$ & $0.10 \pm 0.00$ & $0.18 \pm 0.00$ & $0.20 \pm 0.01$ & $0.22 \pm 0.01$ \\
\hline $\mathrm{S}^{++} / \mathrm{H}^{+}\left(\times 10^{6}\right)$ & $2.55 \pm 0.08$ & $1.57 \pm 0.06$ & $1.92 \pm 0.11$ & $2.17 \pm 0.09$ \\
\hline ICF & $1.72 \pm 0.04$ & $1.49 \pm 0.03$ & $1.39 \pm 0.04$ & $1.28 \pm 0.03$ \\
\hline $\log (\mathrm{S} / \mathrm{O})$ & $-1.41 \pm 0.02$ & $-1.61 \pm 0.02$ & $-1.57 \pm 0.03$ & $-1.57 \pm 0.02$ \\
\hline $\mathrm{Ar}^{++} / \mathrm{H}^{+}\left(\times 10^{7}\right)$ & $5.45 \pm 0.12$ & $4.35 \pm 0.10$ & $5.11 \pm 0.16$ & $5.29 \pm 0.14$ \\
\hline $\mathrm{Ar}^{+++} / \mathrm{H}^{+}\left(\times 10^{7}\right)$ & $0.55 \pm 0.01$ & $1.13 \pm 0.07$ & & \\
\hline ICF & $1.21 \pm 0.02$ & $1.16 \pm 0.01$ & $1.12 \pm 0.02$ & $1.09 \pm 0.01$ \\
\hline $\log (\mathrm{Ar} / \mathrm{O})$ & $-2.25 \pm 0.01$ & $-2.33 \pm 0.02$ & $-2.28 \pm 0.02$ & $-2.29 \pm 0.02$ \\
\hline \multirow[b]{2}{*}{ Property } & \multicolumn{4}{|c|}{$\begin{array}{c}\text { GALAXY } \\
\text { (ESO PROGRAM) }\end{array}$} \\
\hline & $\begin{array}{c}\text { NGC 346B No.2 } \\
(69 . C-0203(A))\end{array}$ & $\begin{array}{c}\text { NGC 346D No.1 } \\
(69 . \mathrm{C}-0203(\mathrm{~A}))\end{array}$ & $\begin{array}{c}\text { NGC 346D No.2 } \\
(69 . C-0203(A))\end{array}$ & $\begin{array}{c}\text { NGC 346D No.3 } \\
(69 . C-0203(A))\end{array}$ \\
\hline$T_{\mathrm{e}}(\mathrm{O}$ III $)(\mathrm{K})$ & $12772 \pm 112$ & $13253 \pm 126$ & $13332 \pm 123$ & $12741 \pm 116$ \\
\hline$T_{\mathrm{e}}\left(\mathrm{O}_{\text {II }}\right)(\mathrm{K})$ & $12470 \pm 127$ & $12831 \pm 144$ & $12888 \pm 141$ & $12446 \pm 132$ \\
\hline$T_{\mathrm{e}}\left(\mathrm{S}_{\text {III }}\right)(\mathrm{K})$ & $12115 \pm 93$ & $12704 \pm 105$ & $12798 \pm 102$ & $12076 \pm 97$ \\
\hline$N_{\mathrm{e}}(\mathrm{S}$ II $)\left(\mathrm{cm}^{-3}\right)$ & $35 \pm 30$ & $16 \pm 17$ & $10 \pm 10$ & $95 \pm 33$ \\
\hline $\mathrm{O}^{+} / \mathrm{H}^{+}\left(\times 10^{4}\right)$ & $0.15 \pm 0.01$ & $0.23 \pm 0.01$ & $0.11 \pm 0.00$ & $0.17 \pm 0.01$ \\
\hline $\mathrm{O}^{++} / \mathrm{H}^{+}\left(\times 10^{4}\right)$ & $0.88 \pm 0.02$ & $0.72 \pm 0.02$ & $0.78 \pm 0.02$ & $0.81 \pm 0.02$ \\
\hline $\mathrm{O} / \mathrm{H}\left(\times 10^{4}\right)$ & $1.03 \pm 0.02$ & $0.95 \pm 0.02$ & $0.89 \pm 0.02$ & $0.98 \pm 0.02$ \\
\hline $12+\log (\mathrm{O} / \mathrm{H})$ & $8.01 \pm 0.01$ & $7.98 \pm 0.01$ & $7.95 \pm 0.01$ & $7.99 \pm 0.01$ \\
\hline $\mathrm{N}^{+} / \mathrm{H}^{+}\left(\times 10^{6}\right)$ & $0.29 \pm 0.01$ & $0.63 \pm 0.03$ & $0.26 \pm 0.01$ & $0.47 \pm 0.02$ \\
\hline ICF & $6.26 \pm 0.23$ & $4.16 \pm 0.15$ & $7.15 \pm 0.28$ & $5.51 \pm 0.20$ \\
\hline $\log (\mathrm{N} / \mathrm{O})$ & $-1.75 \pm 0.03$ & $-1.56 \pm 0.03$ & $-1.68 \pm 0.03$ & $-1.58 \pm 0.03$ \\
\hline $\mathrm{Ne}^{++} / \mathrm{H}^{+}\left(\times 10^{5}\right)$ & $1.73 \pm 0.05$ & $1.38 \pm 0.05$ & $1.48 \pm 0.05$ & $1.62 \pm 0.05$ \\
\hline ICF & $1.06 \pm 0.02$ & $1.15 \pm 0.02$ & $1.05 \pm 0.02$ & $1.08 \pm 0.02$ \\
\hline $\log (\mathrm{Ne} / \mathrm{O})$ & $-0.75 \pm 0.02$ & $-0.78 \pm 0.02$ & $-0.76 \pm 0.02$ & $-0.75 \pm 0.02$ \\
\hline $\mathrm{S}^{+} / \mathrm{H}^{+}\left(\times 10^{6}\right)$ & $0.09 \pm 0.00$ & $0.26 \pm 0.01$ & $0.13 \pm 0.00$ & $0.19 \pm 0.00$ \\
\hline $\mathrm{S}^{++} / \mathrm{H}^{+}\left(\times 10^{6}\right)$ & $1.58 \pm 0.06$ & $1.67 \pm 0.06$ & $1.67 \pm 0.06$ & $2.36 \pm 0.09$ \\
\hline ICF & $1.49 \pm 0.04$ & $1.21 \pm 0.02$ & $1.55 \pm 0.04$ & $1.38 \pm 0.03$ \\
\hline $\log (\mathrm{S} / \mathrm{O})$ & $-1.62 \pm 0.02$ & $-1.65 \pm 0.02$ & $-1.50 \pm 0.02$ & $-1.45 \pm 0.02$ \\
\hline $\mathrm{Ar}^{++} / \mathrm{H}^{+}\left(\times 10^{7}\right)$ & $4.34 \pm 0.10$ & $4.08 \pm 0.10$ & $4.12 \pm 0.10$ & $5.27 \pm 0.13$ \\
\hline $\mathrm{Ar}^{+++} / \mathrm{H}^{+}\left(\times 10^{7}\right)$ & $1.05 \pm 0.10$ & $\ldots$ & $\ldots$ & $0.64 \pm 0.10$ \\
\hline ICF & $1.17 \pm 0.01$ & $1.09 \pm 0.01$ & $1.22 \pm 0.02$ & $1.14 \pm 0.01$ \\
\hline $\log (\mathrm{Ar} / \mathrm{O})$ & $-2.31 \pm 0.02$ & $-2.33 \pm 0.02$ & $-2.25 \pm 0.02$ & $-2.21 \pm 0.02$ \\
\hline
\end{tabular}


Table 7. continued.

\begin{tabular}{|c|c|c|c|c|}
\hline \multirow[b]{2}{*}{ Property } & \multicolumn{4}{|c|}{$\begin{array}{c}\text { GALAXY } \\
\text { (ESO PROGRAM) }\end{array}$} \\
\hline & $\begin{array}{c}\text { NGC 346D No.4 } \\
(69 . \mathrm{C}-0203(\mathrm{~A}))\end{array}$ & $\begin{array}{c}\text { NGC 456A No.1 } \\
(69 . C-0203(\mathrm{~A}))\end{array}$ & $\begin{array}{l}\text { NGC 456A No.2 } \\
(69 . C-0203(A))\end{array}$ & $\begin{array}{c}\text { NGC 456A No.3 } \\
(69 . C-0203(A))\end{array}$ \\
\hline$T_{\mathrm{e}}(\mathrm{O}$ III) $(\mathrm{K})$ & $13036 \pm 121$ & $12308 \pm 108$ & $12269 \pm 139$ & $12134 \pm 117$ \\
\hline$T_{\mathrm{e}}(\mathrm{O}$ II $)(\mathrm{K})$ & $12672 \pm 137$ & $12096 \pm 122$ & $12063 \pm 156$ & $11948 \pm 131$ \\
\hline$T_{\mathrm{e}}(\mathrm{S}$ III $)(\mathrm{K})$ & $12442 \pm 100$ & $11524 \pm 90$ & $11473 \pm 115$ & $11296 \pm 97$ \\
\hline$N_{\mathrm{e}}(\mathrm{S}$ II $)\left(\mathrm{cm}^{-3}\right)$ & $39 \pm 30$ & $238 \pm 39$ & $46 \pm 34$ & $100 \pm 34$ \\
\hline $\mathrm{O}^{+} / \mathrm{H}^{+}\left(\times 10^{4}\right)$ & $0.16 \pm 0.01$ & $0.43 \pm 0.02$ & $0.28 \pm 0.01$ & $0.34 \pm 0.01$ \\
\hline $\mathrm{O}^{++} / \mathrm{H}^{+}\left(\times 10^{4}\right)$ & $0.76 \pm 0.02$ & $0.72 \pm 0.02$ & $0.82 \pm 0.03$ & $0.78 \pm 0.02$ \\
\hline $\mathrm{O} / \mathrm{H}\left(\times 10^{4}\right)$ & $0.92 \pm 0.02$ & $1.15 \pm 0.03$ & $1.10 \pm 0.03$ & $1.12 \pm 0.03$ \\
\hline $12+\log (\mathrm{O} / \mathrm{H})$ & $7.96 \pm 0.01$ & $8.06 \pm 0.01$ & $8.04 \pm 0.01$ & $8.05 \pm 0.01$ \\
\hline $\mathrm{N}^{+} / \mathrm{H}^{+}\left(\times 10^{6}\right)$ & $0.39 \pm 0.02$ & $0.94 \pm 0.04$ & $0.62 \pm 0.03$ & $0.59 \pm 0.03$ \\
\hline ICF & $5.45 \pm 0.20$ & $2.90 \pm 0.09$ & $3.96 \pm 0.16$ & $3.47 \pm 0.12$ \\
\hline $\log (\mathrm{N} / \mathrm{O})$ & $-1.63 \pm 0.03$ & $-1.63 \pm 0.02$ & $-1.65 \pm 0.03$ & $-1.74 \pm 0.03$ \\
\hline $\mathrm{Ne}^{++} / \mathrm{H}^{+}\left(\times 10^{5}\right)$ & $1.54 \pm 0.05$ & $1.23 \pm 0.04$ & $1.52 \pm 0.06$ & $1.32 \pm 0.05$ \\
\hline $\mathrm{ICF}$ & $1.09 \pm 0.02$ & $1.33 \pm 0.03$ & $1.18 \pm 0.03$ & $1.23 \pm 0.03$ \\
\hline $\log (\mathrm{Ne} / \mathrm{O})$ & $-0.74 \pm 0.02$ & $-0.84 \pm 0.02$ & $-0.79 \pm 0.02$ & $-0.84 \pm 0.02$ \\
\hline $\mathrm{S}^{+} / \mathrm{H}^{+}\left(\times 10^{6}\right)$ & $0.18 \pm 0.00$ & $0.27 \pm 0.01$ & $0.18 \pm 0.01$ & $0.17 \pm 0.00$ \\
\hline $\mathrm{S}^{++} / \mathrm{H}^{+}\left(\times 10^{6}\right)$ & $1.92 \pm 0.07$ & $2.38 \pm 0.08$ & $2.02 \pm 0.10$ & $2.53 \pm 0.10$ \\
\hline ICF & $1.36 \pm 0.03$ & $1.08 \pm 0.01$ & $1.20 \pm 0.03$ & $1.14 \pm 0.02$ \\
\hline $\log (\mathrm{S} / \mathrm{O})$ & $-1.51 \pm 0.02$ & $-1.60 \pm 0.02$ & $-1.62 \pm 0.03$ & $-1.56 \pm 0.02$ \\
\hline $\mathrm{Cl}^{++} / \mathrm{H}^{+}\left(\times 10^{8}\right)$ & $\ldots$ & $3.89 \pm 0.24$ & $3.55 \pm 0.40$ & $2.68 \pm 0.24$ \\
\hline $\mathrm{ICF}$ & $\ldots$ & $1.18 \pm 0.08$ & $1.22 \pm 0.15$ & $1.19 \pm 0.11$ \\
\hline $\log (\mathrm{Cl} / \mathrm{O})$ & $\ldots$ & $-3.40 \pm 0.04$ & $-3.41 \pm 0.07$ & $-3.55 \pm 0.06$ \\
\hline $\mathrm{Ar}^{++} / \mathrm{H}^{+}\left(\times 10^{7}\right)$ & $4.40 \pm 0.11$ & $5.48 \pm 0.13$ & $5.31 \pm 0.16$ & $5.52 \pm 0.15$ \\
\hline $\mathrm{Ar}^{+++} / \mathrm{H}^{+}\left(\times 10^{7}\right)$ & $0.80 \pm 0.09$ & & & \\
\hline ICF & $1.14 \pm 0.01$ & $1.07 \pm 0.01$ & $1.08 \pm 0.01$ & $1.07 \pm 0.01$ \\
\hline $\log (\mathrm{Ar} / \mathrm{O})$ & $-2.26 \pm 0.02$ & $-2.29 \pm 0.01$ & $-2.28 \pm 0.02$ & $-2.28 \pm 0.02$ \\
\hline $\mathrm{Fe}^{++} / \mathrm{H}^{+}\left(\times 10^{6}\right)$ & $\ldots$ & $0.16 \pm 0.02$ & $\ldots$ & $0.11 \pm 0.02$ \\
\hline ICF & $\ldots$ & $3.73 \pm 0.14$ & $\ldots$ & $4.54 \pm 0.18$ \\
\hline $\log (\mathrm{Fe} / \mathrm{O})$ & $\ldots$ & $-2.29 \pm 0.05$ & $\ldots$ & $-2.34 \pm 0.06$ \\
\hline \multirow{3}{*}{ Property } & \multicolumn{4}{|c|}{$\begin{array}{c}\text { GaLAXY } \\
\text { (ESO PROGRAM) }\end{array}$} \\
\hline & UM 420 No. 2 & CAM 0357-3915 & TOL 0513-393 & NGC 6822V No.1 \\
\hline & (69.C-0203(A)) & $(69 . \mathrm{C}-0203(\mathrm{~A}))$ & $(69 . \mathrm{C}-0203(\mathrm{~A}))$ & (69.C-0203(A)) \\
\hline$T_{\mathrm{e}}\left(\mathrm{O}_{\text {III }}\right)(\mathrm{K})$ & $12328 \pm 440$ & $15401 \pm 193$ & $15160 \pm 144$ & $11686 \pm 135$ \\
\hline$T_{\mathrm{e}}(\mathrm{O}$ II) $(\mathrm{K})$ & $12112 \pm 496$ & $14099 \pm 227$ & $13985 \pm 169$ & $12119 \pm 151$ \\
\hline$T_{\mathrm{e}}\left(\mathrm{S}_{\text {III }}\right)(\mathrm{K})$ & $11550 \pm 365$ & $15025 \pm 160$ & $14790 \pm 120$ & $11828 \pm 112$ \\
\hline$N_{\mathrm{e}}(\mathrm{S}$ II $)\left(\mathrm{cm}^{-3}\right)$ & $93 \pm 54$ & $254 \pm 76$ & $196 \pm 46$ & $86 \pm 48$ \\
\hline $\mathrm{O}^{+} / \mathrm{H}^{+}\left(\times 10^{4}\right)$ & $0.41 \pm 0.05$ & $0.09 \pm 0.00$ & $0.06 \pm 0.00$ & $0.17 \pm 0.01$ \\
\hline $\mathrm{O}^{++} / \mathrm{H}^{+}\left(\times 10^{4}\right)$ & $1.02 \pm 0.11$ & $0.68 \pm 0.02$ & $0.80 \pm 0.02$ & $1.24 \pm 0.05$ \\
\hline $\mathrm{O}^{+++} / \mathrm{H}^{+}\left(\times 10^{6}\right)$ & ... & $1.50 \pm 0.14$ & $0.94 \pm 0.07$ & \\
\hline $\mathrm{O} / \mathrm{H}\left(\times 10^{4}\right)$ & $1.43 \pm 0.12$ & $0.79 \pm 0.02$ & $0.86 \pm 0.02$ & $1.41 \pm 0.05$ \\
\hline $12+\log (\mathrm{O} / \mathrm{H})$ & $8.16 \pm 0.04$ & $7.90 \pm 0.01$ & $7.94 \pm 0.01$ & $8.15 \pm 0.01$ \\
\hline $\mathrm{N}^{+} / \mathrm{H}^{+}\left(\times 10^{6}\right)$ & $1.28 \pm 0.18$ & $0.39 \pm 0.02$ & $0.28 \pm 0.01$ & $0.38 \pm 0.02$ \\
\hline ICF & $3.69 \pm 0.40$ & $7.70 \pm 0.38$ & $13.34 \pm 0.56$ & $7.32 \pm 0.33$ \\
\hline $\log (\mathrm{N} / \mathrm{O})$ & $-1.48 \pm 0.08$ & $-1.43 \pm 0.04$ & $-1.37 \pm 0.03$ & $-1.71 \pm 0.03$ \\
\hline $\mathrm{Ne}^{++} / \mathrm{H}^{+}\left(\times 10^{5}\right)$ & $2.41 \pm 0.28$ & $1.28 \pm 0.05$ & $1.12 \pm 0.03$ & $2.51 \pm 0.11$ \\
\hline ICF & $1.25 \pm 0.11$ & $1.05 \pm 0.02$ & $1.01 \pm 0.02$ & $1.03 \pm 0.04$ \\
\hline $\log (\mathrm{Ne} / \mathrm{O})$ & $-0.68 \pm 0.07$ & $-0.77 \pm 0.02$ & $-0.88 \pm 0.02$ & $-0.74 \pm 0.03$ \\
\hline $\mathrm{S}^{+} / \mathrm{H}^{+}\left(\times 10^{6}\right)$ & $0.51 \pm 0.04$ & $0.15 \pm 0.01$ & $0.10 \pm 0.00$ & $0.11 \pm 0.00$ \\
\hline $\mathrm{S}^{++} / \mathrm{H}^{+}\left(\times 10^{6}\right)$ & $\ldots$ & $0.67 \pm 0.05$ & $0.66 \pm 0.03$ & $2.20 \pm 0.10$ \\
\hline ICF & $\ldots$ & $1.57 \pm 0.05$ & $2.29 \pm 0.08$ & $1.82 \pm 0.07$ \\
\hline
\end{tabular}


Table 7. continued.

\begin{tabular}{|c|c|c|c|c|}
\hline \multirow[b]{2}{*}{ Property } & \multicolumn{4}{|c|}{$\begin{array}{c}\text { GALAXY } \\
\text { (ESO PROGRAM) }\end{array}$} \\
\hline & $\begin{array}{l}\text { UM 420 No.2 } \\
(69 . \mathrm{C}-0203(\mathrm{~A}))\end{array}$ & $\begin{array}{c}\text { CAM 0357-3915 } \\
(69 . C-0203(A))\end{array}$ & $\begin{array}{l}\text { TOL 0513-393 } \\
(69 . \mathrm{C}-0203(\mathrm{~A}))\end{array}$ & $\begin{array}{l}\text { NGC 6822V No.1 } \\
(69 . C-0203(A))\end{array}$ \\
\hline $\log (\mathrm{S} / \mathrm{O})$ & $\ldots$ & $-1.81 \pm 0.03$ & $-1.69 \pm 0.02$ & $-1.53 \pm 0.03$ \\
\hline $\mathrm{Cl}^{++} / \mathrm{H}^{+}\left(\times 10^{8}\right)$ & $\ldots$ & $1.82 \pm 0.34$ & $1.36 \pm 0.17$ & $3.52 \pm 0.43$ \\
\hline ICF & $\ldots$ & $1.73 \pm 0.45$ & $2.56 \pm 0.63$ & $1.55 \pm 0.21$ \\
\hline $\log (\mathrm{Cl} / \mathrm{O})$ & $\ldots$ & $-3.40 \pm 0.14$ & $-3.39 \pm 0.12$ & $-3.41 \pm 0.08$ \\
\hline $\mathrm{Ar}^{++} / \mathrm{H}^{+}\left(\times 10^{7}\right)$ & $4.53 \pm 0.41$ & $1.50 \pm 0.06$ & $1.80 \pm 0.05$ & $6.00 \pm 0.16$ \\
\hline $\mathrm{Ar}^{+++} / \mathrm{H}^{+}\left(\times 10^{7}\right)$ & $\ldots$ & $1.28 \pm 0.14$ & $1.48 \pm 0.07$ & $0.77 \pm 0.14$ \\
\hline ICF & $1.06 \pm 0.03$ & $1.26 \pm 0.02$ & $1.57 \pm 0.03$ & $1.19 \pm 0.02$ \\
\hline $\log (\mathrm{Ar} / \mathrm{O})$ & $-2.47 \pm 0.06$ & $-2.62 \pm 0.05$ & $-2.49 \pm 0.02$ & $-2.30 \pm 0.02$ \\
\hline $\mathrm{Fe}^{++} / \mathrm{H}^{+}\left(\times 10^{6}\right)$ & $\ldots$ & $0.17 \pm 0.03$ & $0.08 \pm 0.01$ & $\ldots$ \\
\hline ICF & $\ldots$ & $11.02 \pm 0.61$ & $19.81 \pm 0.89$ & $\ldots$ \\
\hline $\log (\mathrm{Fe} / \mathrm{O})$ & $\ldots$ & $-1.63 \pm 0.08$ & $-1.73 \pm 0.07$ & $\ldots$ \\
\hline \multirow[b]{2}{*}{ Property } & \multicolumn{4}{|c|}{$\begin{array}{c}\text { GALAXY } \\
\text { (ESO PROGRAM) }\end{array}$} \\
\hline & $\begin{array}{c}\text { NGC 6822V No.2 } \\
(69 . \mathrm{C}-0203(\mathrm{~A}))\end{array}$ & $\begin{array}{l}\text { TOL 2138-405 No.1 } \\
(69 . \mathrm{C}-0203(\mathrm{~A}))\end{array}$ & $\begin{array}{l}\text { TOL 2146-391 No.1 } \\
\text { (69.C-0203(A)) }\end{array}$ & $\begin{array}{c}\text { TOL 2146-391 No.2 } \\
(69 . \mathrm{C}-0203(\mathrm{~A}))\end{array}$ \\
\hline$T_{\mathrm{e}}(\mathrm{O}$ III $)(\mathrm{K})$ & $12051 \pm 188$ & $13129 \pm 154$ & $16005 \pm 198$ & $16529 \pm 241$ \\
\hline$T_{\mathrm{e}}(\mathrm{O}$ II $)(\mathrm{K})$ & $11876 \pm 212$ & $12741 \pm 175$ & $14354 \pm 235$ & $14539 \pm 289$ \\
\hline$T_{\mathrm{e}}\left(\mathrm{S}_{\text {III }}\right)(\mathrm{K})$ & $11185 \pm 156$ & $12555 \pm 128$ & $15587 \pm 164$ & $16042 \pm 200$ \\
\hline$N_{\mathrm{e}}(\mathrm{S}$ II $)\left(\mathrm{cm}^{-3}\right)$ & $54 \pm 45$ & $349 \pm 56$ & $182 \pm 54$ & $110 \pm 58$ \\
\hline $\mathrm{O}^{+} / \mathrm{H}^{+}\left(\times 10^{4}\right)$ & $0.18 \pm 0.01$ & $0.21 \pm 0.01$ & $0.09 \pm 0.00$ & $0.08 \pm 0.00$ \\
\hline $\mathrm{O}^{++} / \mathrm{H}^{+}\left(\times 10^{4}\right)$ & $1.19 \pm 0.06$ & $1.07 \pm 0.04$ & $0.56 \pm 0.02$ & $0.50 \pm 0.02$ \\
\hline $\mathrm{O}^{+++} / \mathrm{H}^{+}\left(\times 10^{6}\right)$ & $\ldots$ & $2.04 \pm 0.20$ & $1.74 \pm 0.17$ & $1.17 \pm 0.13$ \\
\hline $\mathrm{O} / \mathrm{H}\left(\times 10^{4}\right)$ & $1.36 \pm 0.06$ & $1.31 \pm 0.04$ & $0.66 \pm 0.02$ & $0.59 \pm 0.02$ \\
\hline $12+\log (\mathrm{O} / \mathrm{H})$ & $8.13 \pm 0.02$ & $8.12 \pm 0.01$ & $7.82 \pm 0.01$ & $7.77 \pm 0.01$ \\
\hline $\mathrm{N}^{+} / \mathrm{H}^{+}\left(\times 10^{6}\right)$ & $0.39 \pm 0.03$ & $1.27 \pm 0.07$ & $0.23 \pm 0.01$ & $0.19 \pm 0.01$ \\
\hline ICF & $6.89 \pm 0.43$ & $5.76 \pm 0.24$ & $7.11 \pm 0.36$ & $6.90 \pm 0.41$ \\
\hline $\log (\mathrm{N} / \mathrm{O})$ & $-1.71 \pm 0.05$ & $-1.25 \pm 0.03$ & $-1.61 \pm 0.04$ & $-1.66 \pm 0.04$ \\
\hline $\mathrm{Ne}^{++} / \mathrm{H}^{+}\left(\times 10^{5}\right)$ & $2.36 \pm 0.13$ & $2.28 \pm 0.09$ & $1.07 \pm 0.04$ & $0.98 \pm 0.04$ \\
\hline ICF & $1.04 \pm 0.05$ & $1.10 \pm 0.03$ & $1.07 \pm 0.02$ & $1.07 \pm 0.02$ \\
\hline $\log (\mathrm{Ne} / \mathrm{O})$ & $-0.74 \pm 0.04$ & $-0.72 \pm 0.03$ & $-0.76 \pm 0.02$ & $-0.75 \pm 0.02$ \\
\hline $\mathrm{S}^{+} / \mathrm{H}^{+}\left(\times 10^{6}\right)$ & $0.13 \pm 0.00$ & $0.28 \pm 0.01$ & $0.13 \pm 0.00$ & $0.11 \pm 0.00$ \\
\hline $\mathrm{S}^{++} / \mathrm{H}^{+}\left(\times 10^{6}\right)$ & $2.55 \pm 0.17$ & $1.55 \pm 0.10$ & $0.72 \pm 0.04$ & $0.60 \pm 0.04$ \\
\hline ICF & $1.72 \pm 0.08$ & $1.50 \pm 0.05$ & $1.45 \pm 0.04$ & $1.40 \pm 0.04$ \\
\hline $\log (\mathrm{S} / \mathrm{O})$ & $-1.47 \pm 0.04$ & $-1.68 \pm 0.03$ & $-1.73 \pm 0.03$ & $-1.77 \pm 0.03$ \\
\hline $\mathrm{Ar}^{++} / \mathrm{H}^{+}\left(\times 10^{7}\right)$ & $6.29 \pm 0.24$ & $4.39 \pm 0.14$ & $1.76 \pm 0.06$ & $1.57 \pm 0.06$ \\
\hline $\mathrm{Ar}^{+++} / \mathrm{H}^{+}\left(\times 10^{7}\right)$ & $1.35 \pm 0.22$ & $1.55 \pm 0.21$ & $1.25 \pm 0.11$ & $0.91 \pm 0.11$ \\
\hline ICF & $1.17 \pm 0.03$ & $1.13 \pm 0.02$ & $1.24 \pm 0.02$ & $1.23 \pm 0.02$ \\
\hline $\log (\mathrm{Ar} / \mathrm{O})$ & $-2.27 \pm 0.03$ & $-2.42 \pm 0.03$ & $-2.48 \pm 0.03$ & $-2.48 \pm 0.04$ \\
\hline $\mathrm{Fe}^{++} / \mathrm{H}^{+}\left(\times 10^{6}\right)$ & $\ldots$ & $0.30 \pm 0.04$ & $0.22 \pm 0.03$ & $0.12 \pm 0.02$ \\
\hline ICF & $\ldots$ & $7.90 \pm 0.38$ & $10.13 \pm 0.57$ & $9.84 \pm 0.65$ \\
\hline $\log (\mathrm{Fe} / \mathrm{O})$ & $\ldots$ & $-1.74 \pm 0.06$ & $-1.47 \pm 0.06$ & $-1.71 \pm 0.09$ \\
\hline \multirow{4}{*}{ Property } & \multirow{2}{*}{\multicolumn{2}{|c|}{$\begin{array}{c}\text { GALAXY } \\
\text { (ESO PROGRAM) }\end{array}$}} & & \\
\hline & & & & \\
\hline & TOL 2240-384 & UM 160 No.1 & & \\
\hline & $(69 . \mathrm{C}-0203(\mathrm{~A}))$ & $(69 . \mathrm{C}-0203(\mathrm{~A}))$ & & \\
\hline$T_{\mathrm{e}}(\mathrm{O}$ III $)(\mathrm{K})$ & $15777 \pm 198$ & $13613 \pm 271$ & & \\
\hline$T_{\mathrm{e}}(\mathrm{O}$ II $)(\mathrm{K})$ & $14263 \pm 235$ & $13083 \pm 244$ & & \\
\hline$T_{\mathrm{e}}(\mathrm{S}$ III) $(\mathrm{K})$ & $15380 \pm 165$ & $13129 \pm 225$ & & \\
\hline$N_{\mathrm{e}}(\mathrm{S} \mathrm{II})\left(\mathrm{cm}^{-3}\right)$ & $210 \pm 67$ & $80 \pm 51$ & & \\
\hline $\mathrm{O}^{+} / \mathrm{H}^{+}\left(\times 10^{4}\right)$ & $0.07 \pm 0.00$ & $0.15 \pm 0.01$ & & \\
\hline $\mathrm{O}^{++} / \mathrm{H}^{+}\left(\times 10^{4}\right)$ & $0.62 \pm 0.02$ & $0.79 \pm 0.04$ & & \\
\hline $\mathrm{O}^{+++} / \mathrm{H}^{+}\left(\times 10^{6}\right)$ & $1.22 \pm 0.11$ & $\ldots$ & & \\
\hline
\end{tabular}


Table 7. continued.

\begin{tabular}{|c|c|c|}
\hline \multirow[b]{2}{*}{ Property } & \multicolumn{2}{|c|}{$\begin{array}{c}\text { GALAXY } \\
\text { (ESO PROGRAM) }\end{array}$} \\
\hline & $\begin{array}{l}\text { TOL 2240-384 } \\
(69 . C-0203(A))\end{array}$ & $\begin{array}{c}\text { UM 160 No.1 } \\
(69 . \mathrm{C}-0203(\mathrm{~A}))\end{array}$ \\
\hline$\overline{\mathrm{O} / \mathrm{H}\left(\times 10^{4}\right)}$ & $0.71 \pm 0.02$ & $0.94 \pm 0.05$ \\
\hline $12+\log (\mathrm{O} / \mathrm{H})$ & $7.85 \pm 0.01$ & $7.97 \pm 0.02$ \\
\hline $\mathrm{N}^{+} / \mathrm{H}^{+}\left(\times 10^{6}\right)$ & $0.41 \pm 0.03$ & $0.42 \pm 0.04$ \\
\hline ICF & $9.09 \pm 0.48$ & $5.86 \pm 0.44$ \\
\hline $\log (\mathrm{N} / \mathrm{O})$ & $-1.28 \pm 0.04$ & $-1.58 \pm 0.06$ \\
\hline $\mathrm{Ne}^{++} / \mathrm{H}^{+}\left(\times 10^{5}\right)$ & $1.00 \pm 0.04$ & $1.17 \pm 0.08$ \\
\hline ICF & $1.04 \pm 0.02$ & $1.07 \pm 0.05$ \\
\hline $\log (\mathrm{Ne} / \mathrm{O})$ & $-0.83 \pm 0.02$ & $-0.87 \pm 0.04$ \\
\hline $\mathrm{S}^{+} / \mathrm{H}^{+}\left(\times 10^{6}\right)$ & $0.07 \pm 0.00$ & $0.22 \pm 0.01$ \\
\hline $\mathrm{S}^{++} / \mathrm{H}^{+}\left(\times 10^{6}\right)$ & $0.42 \pm 0.03$ & $1.39 \pm 0.11$ \\
\hline ICF & $1.67 \pm 0.06$ & $1.41 \pm 0.06$ \\
\hline $\log (\mathrm{S} / \mathrm{O})$ & $-1.94 \pm 0.04$ & $-1.62 \pm 0.04$ \\
\hline $\mathrm{Ar}^{++} / \mathrm{H}^{+}\left(\times 10^{7}\right)$ & $0.93 \pm 0.04$ & $2.95 \pm 0.14$ \\
\hline $\mathrm{Ar}^{+++} / \mathrm{H}^{+}\left(\times 10^{7}\right)$ & $1.35 \pm 0.14$ & \\
\hline ICF & $1.34 \pm 0.03$ & $1.16 \pm 0.03$ \\
\hline $\log (\mathrm{Ar} / \mathrm{O})$ & $-2.75 \pm 0.07$ & $-2.44 \pm 0.03$ \\
\hline
\end{tabular}

Table 8. Ionic and total heavy element abundances (FORS medium-resolution observations).

\begin{tabular}{|c|c|c|c|c|}
\hline \multirow[b]{2}{*}{ Property } & \multicolumn{4}{|c|}{$\begin{array}{c}\text { GaLAXY } \\
\text { (ESO PROGRAM) }\end{array}$} \\
\hline & $\begin{array}{c}\text { NGC 346A No.1 } \\
(69 . C-0203(\mathrm{~A}))\end{array}$ & $\begin{array}{c}\text { NGC 346A No.2 } \\
(69 . C-0203(\mathrm{~A}))\end{array}$ & $\begin{array}{c}\text { NGC 346A No.3 } \\
(69 . C-0203(\mathrm{~A}))\end{array}$ & $\begin{array}{l}\text { NGC 346B No.1 } \\
(69 . \mathrm{C}-0203(\mathrm{~A}))\end{array}$ \\
\hline$\overline{T_{\mathrm{e}}\left(\mathrm{O}_{\text {III }}\right)(\mathrm{K})}$ & $12955 \pm 97$ & $12563 \pm 93$ & $12666 \pm 106$ & $12594 \pm 94$ \\
\hline$T_{\mathrm{e}}(\mathrm{O}$ II $)(\mathrm{K})$ & $12611 \pm 110$ & $12305 \pm 105$ & $12387 \pm 120$ & $12330 \pm 87$ \\
\hline$T_{\mathrm{e}}(\mathrm{S}$ III $)(\mathrm{K})$ & $12343 \pm 80$ & $11852 \pm 77$ & $11982 \pm 88$ & $11892 \pm 78$ \\
\hline$N_{\mathrm{e}}(\mathrm{S}$ II $)\left(\mathrm{cm}^{-3}\right)$ & $10 \pm 10$ & $42 \pm 32$ & $51 \pm 37$ & $65 \pm 35$ \\
\hline $\mathrm{O}^{+} / \mathrm{H}^{+}\left(\times 10^{4}\right)$ & $0.14 \pm 0.00$ & $0.17 \pm 0.01$ & $0.20 \pm 0.01$ & $0.27 \pm 0.01$ \\
\hline $\mathrm{O}^{++} / \mathrm{H}^{+}\left(\times 10^{4}\right)$ & $0.89 \pm 0.02$ & $0.92 \pm 0.02$ & $0.85 \pm 0.02$ & $0.86 \pm 0.02$ \\
\hline $\mathrm{O} / \mathrm{H}\left(\times 10^{4}\right)$ & $1.03 \pm 0.02$ & $1.08 \pm 0.02$ & $1.05 \pm 0.02$ & $1.13 \pm 0.02$ \\
\hline $12+\log (\mathrm{O} / \mathrm{H})$ & $8.01 \pm 0.01$ & $8.04 \pm 0.01$ & $8.02 \pm 0.01$ & $8.05 \pm 0.01$ \\
\hline $\mathrm{N}^{+} / \mathrm{H}^{+}\left(\times 10^{6}\right)$ & $0.26 \pm 0.01$ & $0.44 \pm 0.02$ & $0.56 \pm 0.02$ & $0.60 \pm 0.02$ \\
\hline ICF & $6.58 \pm 0.21$ & $6.02 \pm 0.18$ & $5.04 \pm 0.16$ & $4.16 \pm 0.12$ \\
\hline $\log (\mathrm{N} / \mathrm{O})$ & $-1.78 \pm 0.02$ & $-1.61 \pm 0.02$ & $-1.57 \pm 0.02$ & $-1.66 \pm 0.02$ \\
\hline $\mathrm{Ne}^{++} / \mathrm{H}^{+}\left(\times 10^{5}\right)$ & $1.93 \pm 0.05$ & $1.91 \pm 0.05$ & $1.92 \pm 0.06$ & $1.90 \pm 0.05$ \\
\hline ICF & $1.06 \pm 0.02$ & $1.07 \pm 0.02$ & $1.10 \pm 0.02$ & $1.16 \pm 0.02$ \\
\hline $\log (\mathrm{Ne} / \mathrm{O})$ & $-0.71 \pm 0.02$ & $-0.73 \pm 0.02$ & $-0.69 \pm 0.02$ & $-0.71 \pm 0.02$ \\
\hline $\mathrm{S}^{+} / \mathrm{H}^{+}\left(\times 10^{6}\right)$ & $0.09 \pm 0.00$ & $0.17 \pm 0.00$ & $0.19 \pm 0.00$ & $0.22 \pm 0.00$ \\
\hline $\mathrm{S}^{++} / \mathrm{H}^{+}\left(\times 10^{6}\right)$ & $1.84 \pm 0.05$ & $1.48 \pm 0.04$ & $1.82 \pm 0.06$ & $2.17 \pm 0.06$ \\
\hline $\mathrm{ICF}$ & $1.54 \pm 0.03$ & $1.48 \pm 0.03$ & $1.33 \pm 0.03$ & $1.23 \pm 0.02$ \\
\hline $\log (\mathrm{S} / \mathrm{O})$ & $-1.54 \pm 0.02$ & $-1.65 \pm 0.02$ & $-1.59 \pm 0.02$ & $-1.59 \pm 0.02$ \\
\hline $\mathrm{Cl}^{++} / \mathrm{H}^{+}\left(\times 10^{8}\right)$ & $3.57 \pm 0.12$ & $2.67 \pm 0.14$ & $3.04 \pm 0.24$ & $2.74 \pm 0.15$ \\
\hline $\mathrm{ICF}$ & $1.52 \pm 0.20$ & $1.44 \pm 0.18$ & $1.33 \pm 0.16$ & $1.23 \pm 0.11$ \\
\hline $\log (\mathrm{Cl} / \mathrm{O})$ & $-3.28 \pm 0.06$ & $-3.45 \pm 0.06$ & $-3.41 \pm 0.06$ & $-3.53 \pm 0.05$ \\
\hline $\mathrm{Ar}^{++} / \mathrm{H}^{+}\left(\times 10^{7}\right)$ & $4.62 \pm 0.10$ & $5.07 \pm 0.11$ & $4.88 \pm 0.11$ & $4.57 \pm 0.09$ \\
\hline $\mathrm{Ar}^{+++} / \mathrm{H}^{+}\left(\times 10^{7}\right)$ & $0.32 \pm 0.03$ & $1.02 \pm 0.05$ & $0.78 \pm 0.08$ & \\
\hline ICF & $1.18 \pm 0.01$ & $1.15 \pm 0.01$ & $1.11 \pm 0.01$ & $1.08 \pm 0.01$ \\
\hline $\log (\mathrm{Ar} / \mathrm{O})$ & $-2.28 \pm 0.01$ & $-2.34 \pm 0.01$ & $-2.29 \pm 0.02$ & $-2.32 \pm 0.01$ \\
\hline $\mathrm{Fe}^{++} / \mathrm{H}^{+}\left(\times 10^{6}\right)(4658)$ & $\ldots$ & $\ldots$ & $\ldots$ & $\ldots$ \\
\hline $\mathrm{Fe}^{++} / \mathrm{H}^{+}\left(\times 10^{6}\right)(4988)$ & $\ldots$ & $\ldots$ & $\ldots$ & $0.20 \pm 0.01$ \\
\hline
\end{tabular}


Table 8. continued.

\begin{tabular}{|c|c|c|c|c|}
\hline \multirow[b]{2}{*}{ Property } & \multicolumn{4}{|c|}{$\begin{array}{c}\text { GALAXY } \\
\text { (ESO PROGRAM) }\end{array}$} \\
\hline & $\begin{array}{l}\text { NGC 346A No.1 } \\
(69 . C-0203(A))\end{array}$ & $\begin{array}{c}\text { NGC 346A No.2 } \\
(69 . C-0203(\mathrm{~A}))\end{array}$ & $\begin{array}{c}\text { NGC 346A No.3 } \\
(69 . C-0203(\mathrm{~A}))\end{array}$ & $\begin{array}{l}\text { NGC 346B No.1 } \\
(69 . C-0203(A))\end{array}$ \\
\hline $\mathrm{ICF}$ & $\ldots$ & $\ldots$ & $\ldots$ & 5.758 \\
\hline $\log (\mathrm{Fe} / \mathrm{O})(4658)$ & $\ldots$ & $\ldots$ & $\ldots$ & $\ldots$ \\
\hline $\log (\mathrm{Fe} / \mathrm{O})(4988)$ & $\ldots$ & $\ldots$ & $\ldots$ & $-2.01 \pm 0.03$ \\
\hline \multirow[b]{2}{*}{ Property } & \multicolumn{4}{|c|}{$\begin{array}{c}\text { GALAXY } \\
\text { (ESO PROGRAM) }\end{array}$} \\
\hline & $\begin{array}{c}\text { NGC 346B No.2 } \\
(69 . \mathrm{C}-0203(\mathrm{~A}))\end{array}$ & $\begin{array}{c}\text { NGC 346D No.1 } \\
(69 . C-0203(\mathrm{~A}))\end{array}$ & $\begin{array}{c}\text { NGC 346D No.2 } \\
(69 . C-0203(A))\end{array}$ & $\begin{array}{c}\text { NGC 346D No.3 } \\
(69 . C-0203(A))\end{array}$ \\
\hline$T_{\mathrm{e}}(\mathrm{O}$ III) $(\mathrm{K})$ & $12560 \pm 94$ & $12911 \pm 96$ & $12547 \pm 92$ & $12837 \pm 96$ \\
\hline$T_{\mathrm{e}}\left(\mathrm{O}_{\text {III }}(\mathrm{K})\right.$ & $12302 \pm 106$ & $12578 \pm 109$ & $12292 \pm 104$ & $12521 \pm 108$ \\
\hline$T_{\mathrm{e}}\left(\mathrm{S}_{\text {III }}\right)(\mathrm{K})$ & $11847 \pm 78$ & $12228 \pm 79$ & $11831 \pm 77$ & $12196 \pm 79$ \\
\hline$N_{\mathrm{e}}(\mathrm{S}$ II $)\left(\mathrm{cm}^{-3}\right)$ & $28 \pm 23$ & $13 \pm 12$ & $12 \pm 11$ & $146 \pm 39$ \\
\hline $\mathrm{O}^{+} / \mathrm{H}^{+}\left(\times 10^{4}\right)$ & $0.15 \pm 0.00$ & $0.29 \pm 0.01$ & $0.16 \pm 0.00$ & $0.19 \pm 0.01$ \\
\hline $\mathrm{O}^{++} / \mathrm{H}^{+}\left(\times 10^{4}\right)$ & $0.90 \pm 0.02$ & $0.77 \pm 0.02$ & $0.92 \pm 0.02$ & $0.81 \pm 0.02$ \\
\hline $\mathrm{O} / \mathrm{H}\left(\times 10^{4}\right)$ & $1.05 \pm 0.02$ & $1.06 \pm 0.02$ & $1.08 \pm 0.02$ & $1.00 \pm 0.02$ \\
\hline $12+\log (\mathrm{O} / \mathrm{H})$ & $8.02 \pm 0.01$ & $8.03 \pm 0.01$ & $8.03 \pm 0.01$ & $8.00 \pm 0.01$ \\
\hline $\mathrm{N}^{+} / \mathrm{H}^{+}\left(\times 10^{6}\right)$ & $0.32 \pm 0.01$ & $0.72 \pm 0.02$ & $0.37 \pm 0.01$ & $0.51 \pm 0.02$ \\
\hline ICF & $6.44 \pm 0.20$ & $3.78 \pm 0.10$ & $6.39 \pm 0.20$ & $4.99 \pm 0.15$ \\
\hline $\log (\mathrm{N} / \mathrm{O})$ & $-1.70 \pm 0.02$ & $-1.59 \pm 0.02$ & $-1.66 \pm 0.02$ & $-1.60 \pm 0.02$ \\
\hline $\mathrm{Ne}^{++} / \mathrm{H}^{+}\left(\times 10^{5}\right)$ & $1.81 \pm 0.05$ & $1.65 \pm 0.04$ & $1.86 \pm 0.05$ & $1.75 \pm 0.05$ \\
\hline ICF & $1.06 \pm 0.02$ & $1.19 \pm 0.02$ & $1.06 \pm 0.02$ & $1.10 \pm 0.02$ \\
\hline $\log (\mathrm{Ne} / \mathrm{O})$ & $-0.74 \pm 0.02$ & $-0.73 \pm 0.02$ & $-0.74 \pm 0.02$ & $-0.71 \pm 0.02$ \\
\hline $\mathrm{S}^{+} / \mathrm{H}^{+}\left(\times 10^{6}\right)$ & $0.10 \pm 0.00$ & $0.28 \pm 0.01$ & $0.15 \pm 0.00$ & $0.19 \pm 0.00$ \\
\hline $\mathrm{S}^{++} / \mathrm{H}^{+}\left(\times 10^{6}\right)$ & $2.21 \pm 0.06$ & $1.87 \pm 0.05$ & $2.09 \pm 0.06$ & $2.13 \pm 0.06$ \\
\hline ICF & $1.52 \pm 0.03$ & $1.18 \pm 0.02$ & $1.53 \pm 0.03$ & $1.32 \pm 0.02$ \\
\hline $\log (\mathrm{S} / \mathrm{O})$ & $-1.47 \pm 0.02$ & $-1.63 \pm 0.01$ & $-1.50 \pm 0.02$ & $-1.52 \pm 0.02$ \\
\hline $\mathrm{Cl}^{++} / \mathrm{H}^{+}\left(\times 10^{8}\right)$ & $2.76 \pm 0.13$ & $3.17 \pm 0.11$ & $3.50 \pm 0.16$ & $3.20 \pm 0.13$ \\
\hline ICF & $1.50 \pm 0.20$ & $1.21 \pm 0.10$ & $1.49 \pm 0.19$ & $1.33 \pm 0.15$ \\
\hline $\log (\mathrm{Cl} / \mathrm{O})$ & $-3.40 \pm 0.06$ & $-3.44 \pm 0.04$ & $-3.31 \pm 0.06$ & $-3.37 \pm 0.05$ \\
\hline $\mathrm{Ar}^{++} / \mathrm{H}^{+}\left(\times 10^{7}\right)$ & $4.54 \pm 0.10$ & $4.47 \pm 0.10$ & $4.84 \pm 0.11$ & $4.73 \pm 0.10$ \\
\hline $\mathrm{Ar}^{+++} / \mathrm{H}^{+}\left(\times 10^{7}\right)$ & $\ldots$ & $0.24 \pm 0.02$ & $0.40 \pm 0.03$ & $\ldots$ \\
\hline ICF & $1.18 \pm 0.01$ & $1.08 \pm 0.01$ & $1.17 \pm 0.01$ & $1.12 \pm 0.01$ \\
\hline $\log (\mathrm{Ar} / \mathrm{O})$ & $-2.29 \pm 0.01$ & $-2.34 \pm 0.01$ & $-2.28 \pm 0.01$ & $-2.28 \pm 0.01$ \\
\hline $\mathrm{Fe}^{++} / \mathrm{H}^{+}\left(\times 10^{6}\right)(4988)$ & $0.29 \pm 0.01$ & $0.07 \pm 0.00$ & $0.10 \pm 0.01$ & $\ldots$ \\
\hline ICF & $9.00 \pm 0.32$ & $4.99 \pm 0.16$ & $8.91 \pm 0.31$ & $\ldots$ \\
\hline $\log (\mathrm{Fe} / \mathrm{O})(4988)$ & $-1.60 \pm 0.03$ & $-2.48 \pm 0.03$ & $-2.10 \pm 0.04$ & $\ldots$ \\
\hline \multirow[b]{2}{*}{ Property } & \multicolumn{4}{|c|}{$\begin{array}{c}\text { GalaXY } \\
\text { (ESO PROGRAM) }\end{array}$} \\
\hline & $\begin{array}{c}\text { NGC 346D No.4 } \\
(69 . C-0203(\mathrm{~A}))\end{array}$ & $\begin{array}{c}\text { NGC 456A No.1 } \\
(69 . C-0203(A))\end{array}$ & $\begin{array}{c}\text { NGC 456A No. } 2 \\
(69 . C-0203(A))\end{array}$ & $\begin{array}{c}\text { NGC 456A No.3 } \\
(69 . \mathrm{C}-0203(\mathrm{~A}))\end{array}$ \\
\hline$T_{\mathrm{e}}\left(\mathrm{O}_{\mathrm{III}}\right)(\mathrm{K})$ & $13017 \pm 99$ & $12281 \pm 88$ & $12029 \pm 94$ & $12084 \pm 88$ \\
\hline$T_{\mathrm{e}}\left(\mathrm{O}_{\mathrm{II}}\right)(\mathrm{K})$ & $12658 \pm 113$ & $12073 \pm 100$ & $11858 \pm 105$ & $11905 \pm 99$ \\
\hline$T_{\mathrm{e}}\left(\mathrm{S}_{\text {III }}\right)(\mathrm{K})$ & $12418 \pm 82$ & $11488 \pm 73$ & $11157 \pm 78$ & $11230 \pm 73$ \\
\hline$N_{\mathrm{e}}(\mathrm{S}$ II $)\left(\mathrm{cm}^{-3}\right)$ & $105 \pm 36$ & $387 \pm 51$ & $87 \pm 36$ & $124 \pm 36$ \\
\hline $\mathrm{O}^{+} / \mathrm{H}^{+}\left(\times 10^{4}\right)$ & $0.20 \pm 0.01$ & $0.45 \pm 0.01$ & $0.27 \pm 0.01$ & $0.34 \pm 0.01$ \\
\hline $\mathrm{O}^{++} / \mathrm{H}^{+}\left(\times 10^{4}\right)$ & $0.79 \pm 0.02$ & $0.70 \pm 0.02$ & $0.86 \pm 0.02$ & $0.78 \pm 0.02$ \\
\hline $\mathrm{O} / \mathrm{H}\left(\times 10^{4}\right)$ & $0.99 \pm 0.02$ & $1.15 \pm 0.02$ & $1.13 \pm 0.02$ & $1.12 \pm 0.02$ \\
\hline $12+\log (\mathrm{O} / \mathrm{H})$ & $8.00 \pm 0.01$ & $8.06 \pm 0.01$ & $8.05 \pm 0.01$ & $8.05 \pm 0.01$ \\
\hline $\mathrm{N}^{+} / \mathrm{H}^{+}\left(\times 10^{6}\right)$ & $0.47 \pm 0.02$ & $0.94 \pm 0.03$ & $0.59 \pm 0.02$ & $0.63 \pm 0.02$ \\
\hline ICF & $4.85 \pm 0.15$ & $2.81 \pm 0.07$ & $4.19 \pm 0.12$ & $3.44 \pm 0.09$ \\
\hline $\log (\mathrm{N} / \mathrm{O})$ & $-1.64 \pm 0.02$ & $-1.64 \pm 0.02$ & $-1.66 \pm 0.02$ & $-1.72 \pm 0.02$ \\
\hline $\mathrm{Ne}^{++} / \mathrm{H}^{+}\left(\times 10^{5}\right)$ & $1.71 \pm 0.05$ & $1.25 \pm 0.03$ & $1.51 \pm 0.04$ & $1.35 \pm 0.04$ \\
\hline ICF & $1.11 \pm 0.02$ & $1.36 \pm 0.02$ & $1.16 \pm 0.02$ & $1.24 \pm 0.02$ \\
\hline
\end{tabular}


Table 8. continued.

\begin{tabular}{|c|c|c|c|c|}
\hline \multirow[b]{2}{*}{ Property } & \multicolumn{4}{|c|}{$\begin{array}{c}\text { GALAXY } \\
\text { (ESO PROGRAM) }\end{array}$} \\
\hline & $\begin{array}{l}\text { NGC 346D No.4 } \\
(69 . \mathrm{C}-0203(\mathrm{~A}))\end{array}$ & $\begin{array}{l}\text { NGC 456A No.1 } \\
(69 . \mathrm{C}-0203(\mathrm{~A}))\end{array}$ & $\begin{array}{c}\text { NGC 456A No.2 } \\
(69 . C-0203(\mathrm{~A}))\end{array}$ & $\begin{array}{c}\text { NGC 456A No.3 } \\
(69 . \mathrm{C}-0203(\mathrm{~A}))\end{array}$ \\
\hline $\log (\mathrm{Ne} / \mathrm{O})$ & $-0.72 \pm 0.02$ & $-0.83 \pm 0.02$ & $-0.81 \pm 0.02$ & $-0.83 \pm 0.02$ \\
\hline $\mathrm{S}^{+} / \mathrm{H}^{+}\left(\times 10^{6}\right)$ & $0.19 \pm 0.00$ & $0.26 \pm 0.01$ & $0.20 \pm 0.00$ & $0.18 \pm 0.00$ \\
\hline $\mathrm{S}^{++} / \mathrm{H}^{+}\left(\times 10^{6}\right)$ & $1.66 \pm 0.05$ & $2.36 \pm 0.07$ & $2.35 \pm 0.08$ & $2.58 \pm 0.08$ \\
\hline ICF & $1.30 \pm 0.02$ & $1.07 \pm 0.01$ & $1.23 \pm 0.02$ & $1.14 \pm 0.02$ \\
\hline $\log (\mathrm{S} / \mathrm{O})$ & $-1.61 \pm 0.02$ & $-1.61 \pm 0.01$ & $-1.55 \pm 0.02$ & $-1.55 \pm 0.02$ \\
\hline $\mathrm{Cl}^{++} / \mathrm{H}^{+}\left(\times 10^{8}\right)$ & $3.00 \pm 0.13$ & $3.20 \pm 0.11$ & $3.27 \pm 0.19$ & $3.25 \pm 0.14$ \\
\hline ICF & $1.32 \pm 0.15$ & $1.18 \pm 0.07$ & $1.23 \pm 0.12$ & $1.19 \pm 0.09$ \\
\hline $\log (\mathrm{Cl} / \mathrm{O})$ & $-3.40 \pm 0.05$ & $-3.48 \pm 0.03$ & $-3.45 \pm 0.05$ & $-3.46 \pm 0.04$ \\
\hline $\mathrm{Ar}^{++} / \mathrm{H}^{+}\left(\times 10^{7}\right)$ & $4.46 \pm 0.10$ & $5.53 \pm 0.12$ & $5.97 \pm 0.14$ & $5.69 \pm 0.13$ \\
\hline $\mathrm{Ar}^{+++} / \mathrm{H}^{+}\left(\times 10^{7}\right)$ & $0.48 \pm 0.04$ & & & . \\
\hline ICF & $1.11 \pm 0.01$ & $1.07 \pm 0.01$ & $1.08 \pm 0.01$ & $1.07 \pm 0.01$ \\
\hline $\log (\mathrm{Ar} / \mathrm{O})$ & $-2.30 \pm 0.01$ & $-2.29 \pm 0.01$ & $-2.24 \pm 0.01$ & $-2.27 \pm 0.01$ \\
\hline $\mathrm{Fe}^{++} / \mathrm{H}^{+}\left(\times 10^{6}\right)(4658)$ & & $0.11 \pm 0.01$ & $0.10 \pm 0.01$ & $0.13 \pm 0.00$ \\
\hline $\mathrm{Fe}^{++} / \mathrm{H}^{+}\left(\times 10^{6}\right)(4988)$ & $0.15 \pm 0.01$ & $0.09 \pm 0.01$ & $0.11 \pm 0.01$ & $0.12 \pm 0.01$ \\
\hline ICF & $6.59 \pm 0.23$ & $3.60 \pm 0.11$ & $5.59 \pm 0.19$ & $4.50 \pm 0.14$ \\
\hline $\log (\mathrm{Fe} / \mathrm{O})(4658)$ & $\ldots$ & $-2.46 \pm 0.03$ & $-2.29 \pm 0.05$ & $-2.29 \pm 0.02$ \\
\hline $\log (\mathrm{Fe} / \mathrm{O})(4988)$ & $-2.01 \pm 0.03$ & $-2.57 \pm 0.03$ & $-2.28 \pm 0.04$ & $-2.30 \pm 0.03$ \\
\hline \multirow{3}{*}{ Property } & \multicolumn{4}{|c|}{$\begin{array}{c}\text { GALAXY } \\
\text { (ESO PROGRAM) }\end{array}$} \\
\hline & UM 420 No.1 & UM 420 No.2 & CAM 0357-3915 & TOL 0513-393 \\
\hline & $(69 . \mathrm{C}-0203(\mathrm{~A}))$ & $(69 . \mathrm{C}-0203(\mathrm{~A}))$ & $(69 . \mathrm{C}-0203(\mathrm{~A}))$ & $(69 . \mathrm{C}-0203(\mathrm{~A}))$ \\
\hline$\overline{T_{\mathrm{e}}(\mathrm{O} \text { III) }(\mathrm{K})}$ & $12149 \pm 386$ & $13659 \pm 287$ & $15124 \pm 148$ & $15131 \pm 136$ \\
\hline$T_{\mathrm{e}}\left(\mathrm{O}_{\text {II }}\right)(\mathrm{K})$ & $11961 \pm 434$ & $13114 \pm 330$ & $13967 \pm 174$ & $13971 \pm 159$ \\
\hline$T_{\mathrm{e}}(\mathrm{S}$ III $)(\mathrm{K})$ & $11315 \pm 321$ & $13181 \pm 239$ & $14754 \pm 123$ & $14761 \pm 113$ \\
\hline$N_{\mathrm{e}}(\mathrm{S}$ II $)\left(\mathrm{cm}^{-3}\right)$ & $49 \pm 47$ & $60 \pm 45$ & $216 \pm 54$ & $273 \pm 54$ \\
\hline $\mathrm{O}^{+} / \mathrm{H}^{+}\left(\times 10^{4}\right)$ & $0.65 \pm 0.08$ & $0.40 \pm 0.03$ & $0.10 \pm 0.00$ & $0.06 \pm 0.00$ \\
\hline $\mathrm{O}^{++} / \mathrm{H}^{+}\left(\times 10^{4}\right)$ & $0.76 \pm 0.07$ & $0.70 \pm 0.04$ & $0.69 \pm 0.02$ & $0.81 \pm 0.02$ \\
\hline $\mathrm{O}^{+++} / \mathrm{H}^{+}\left(\times 10^{6}\right)$ & & $\ldots$ & $1.45 \pm 0.09$ & $0.79 \pm 0.05$ \\
\hline $\mathrm{O} / \mathrm{H}\left(\times 10^{4}\right)$ & $1.41 \pm 0.11$ & $1.10 \pm 0.05$ & $0.80 \pm 0.02$ & $0.88 \pm 0.02$ \\
\hline $12+\log (\mathrm{O} / \mathrm{H})$ & $8.15 \pm 0.03$ & $8.04 \pm 0.02$ & $7.90 \pm 0.01$ & $7.95 \pm 0.01$ \\
\hline $\mathrm{N}^{+} / \mathrm{H}^{+}\left(\times 10^{6}\right)$ & $2.87 \pm 0.35$ & $1.16 \pm 0.10$ & $0.42 \pm 0.02$ & $0.36 \pm 0.02$ \\
\hline ICF & $2.51 \pm 0.24$ & $2.98 \pm 0.19$ & $7.16 \pm 0.28$ & $11.77 \pm 0.46$ \\
\hline $\log (\mathrm{N} / \mathrm{O})$ & $-1.29 \pm 0.07$ & $-1.50 \pm 0.05$ & $-1.43 \pm 0.03$ & $-1.32 \pm 0.03$ \\
\hline $\mathrm{Ne}^{++} / \mathrm{H}^{+}\left(\times 10^{5}\right)$ & $2.00 \pm 0.21$ & $1.88 \pm 0.12$ & $1.33 \pm 0.04$ & $1.40 \pm 0.04$ \\
\hline ICF & $1.56 \pm 0.12$ & $1.30 \pm 0.06$ & $1.06 \pm 0.02$ & $1.01 \pm 0.02$ \\
\hline $\log (\mathrm{Ne} / \mathrm{O})$ & $-0.66 \pm 0.06$ & $-0.65 \pm 0.04$ & $-0.75 \pm 0.02$ & $-0.80 \pm 0.02$ \\
\hline $\mathrm{S}^{+} / \mathrm{H}^{+}\left(\times 10^{6}\right)$ & $0.85 \pm 0.06$ & $0.42 \pm 0.02$ & $0.12 \pm 0.00$ & $0.13 \pm 0.00$ \\
\hline $\mathrm{S}^{++} / \mathrm{H}^{+}\left(\times 10^{6}\right)$ & $2.08 \pm 0.35$ & $1.28 \pm 0.11$ & $0.64 \pm 0.03$ & $0.80 \pm 0.03$ \\
\hline $\mathrm{ICF}$ & $1.03 \pm 0.05$ & $1.09 \pm 0.14$ & $1.52 \pm 0.04$ & $2.12 \pm 0.06$ \\
\hline $\log (\mathrm{S} / \mathrm{O})$ & $-1.67 \pm 0.06$ & $-1.77 \pm 0.04$ & $-1.84 \pm 0.02$ & $-1.65 \pm 0.02$ \\
\hline $\mathrm{Cl}^{++} / \mathrm{H}^{+}\left(\times 10^{8}\right)$ & $\ldots$ & $\ldots$ & $1.36 \pm 0.18$ & $1.64 \pm 0.13$ \\
\hline ICF & $\ldots$ & $\ldots$ & $1.65 \pm 0.33$ & $2.32 \pm 0.51$ \\
\hline $\log (\mathrm{Cl} / \mathrm{O})$ & $\ldots$ & $\ldots$ & $-3.55 \pm 0.10$ & $-3.37 \pm 0.10$ \\
\hline $\mathrm{Fe}^{++} / \mathrm{H}^{+}\left(\times 10^{6}\right)(4658)$ & $0.57 \pm 0.11$ & $0.44 \pm 0.07$ & $0.14 \pm 0.02$ & $0.10 \pm 0.01$ \\
\hline $\mathrm{Fe}^{++} / \mathrm{H}^{+}\left(\times 10^{6}\right)(4988)$ & $0.63 \pm 0.11$ & $0.36 \pm 0.06$ & $0.18 \pm 0.01$ & $0.10 \pm 0.01$ \\
\hline ICF & $3.18 \pm 0.35$ & $3.85 \pm 0.30$ & $10.18 \pm 0.45$ & $17.33 \pm 0.73$ \\
\hline $\log (\mathrm{Fe} / \mathrm{O})(4658)$ & $-1.89 \pm 0.10$ & $-1.81 \pm 0.08$ & $-1.76 \pm 0.07$ & $-1.69 \pm 0.05$ \\
\hline $\log (\mathrm{Fe} / \mathrm{O})(4988)$ & $-1.85 \pm 0.10$ & $-1.90 \pm 0.08$ & $-1.64 \pm 0.04$ & $-1.69 \pm 0.04$ \\
\hline
\end{tabular}


Table 8. continued.

\begin{tabular}{|c|c|c|c|c|}
\hline \multirow[b]{2}{*}{ Property } & \multicolumn{4}{|c|}{$\begin{array}{c}\text { GALAXY } \\
\text { (ESO PROGRAM) }\end{array}$} \\
\hline & $\begin{array}{l}\text { TOL 1214-277 } \\
(65 . \mathrm{N}-0642(\mathrm{~A}))\end{array}$ & $\begin{array}{c}\text { TOL 65 No.1 } \\
(65 . \mathrm{N}-0642(\mathrm{~A}))\end{array}$ & $\begin{array}{c}\text { TOL 65 No.2 } \\
(65 . \mathrm{N}-0642(\mathrm{~A}))\end{array}$ & $\begin{array}{c}\text { NGC 6822V No.1 } \\
(69 . C-0203(\mathrm{~A}))\end{array}$ \\
\hline$T_{\mathrm{e}}(\mathrm{O}$ III $)(\mathrm{K})$ & $19675 \pm 224$ & $17844 \pm 189$ & $17552 \pm 261$ & $11924 \pm 104$ \\
\hline$T_{\mathrm{e}}\left(\mathrm{O}_{\text {II }}\right)(\mathrm{K})$ & $15107 \pm 282$ & $14947 \pm 231$ & $14865 \pm 318$ & $11765 \pm 117$ \\
\hline$T_{\mathrm{e}}(\mathrm{S}$ III) $(\mathrm{K})$ & $18244 \pm 186$ & $17083 \pm 157$ & $16861 \pm 217$ & $11015 \pm 86$ \\
\hline$N_{\mathrm{e}}(\mathrm{S}$ II $)\left(\mathrm{cm}^{-3}\right)$ & $261 \pm 68$ & $169 \pm 40$ & $100 \pm 50$ & $83 \pm 54$ \\
\hline $\mathrm{O}^{+} / \mathrm{H}^{+}\left(\times 10^{4}\right)$ & $0.03 \pm 0.00$ & $0.07 \pm 0.00$ & $0.10 \pm 0.01$ & $0.16 \pm 0.01$ \\
\hline $\mathrm{O}^{++} / \mathrm{H}^{+}\left(\times 10^{4}\right)$ & $0.31 \pm 0.01$ & $0.27 \pm 0.01$ & $0.26 \pm 0.01$ & $1.19 \pm 0.03$ \\
\hline $\mathrm{O}^{+++} / \mathrm{H}^{+}\left(\times 10^{6}\right)$ & $1.97 \pm 0.07$ & $0.26 \pm 0.02$ & $0.91 \pm 0.08$ & $\ldots$ \\
\hline $\mathrm{O} / \mathrm{H}\left(\times 10^{4}\right)$ & $0.36 \pm 0.01$ & $0.34 \pm 0.01$ & $0.36 \pm 0.01$ & $1.35 \pm 0.03$ \\
\hline $12+\log (\mathrm{O} / \mathrm{H})$ & $7.55 \pm 0.01$ & $7.53 \pm 0.01$ & $7.56 \pm 0.01$ & $8.13 \pm 0.01$ \\
\hline $\mathrm{N}^{+} / \mathrm{H}^{+}\left(\times 10^{6}\right)$ & $0.06 \pm 0.00$ & $0.12 \pm 0.01$ & $0.18 \pm 0.01$ & $0.38 \pm 0.02$ \\
\hline ICF & $11.82 \pm 0.66$ & $5.03 \pm 0.02$ & $3.75 \pm 0.22$ & $7.43 \pm 0.27$ \\
\hline $\log (\mathrm{N} / \mathrm{O})$ & $-1.70 \pm 0.04$ & $-1.75 \pm 0.03$ & $-1.74 \pm 0.04$ & $-1.68 \pm 0.03$ \\
\hline $\mathrm{Ne}^{++} / \mathrm{H}^{+}\left(\times 10^{5}\right)$ & $0.45 \pm 0.01$ & $0.54 \pm 0.02$ & $0.55 \pm 0.02$ & $2.45 \pm 0.08$ \\
\hline ICF & $1.05 \pm 0.01$ & $1.08 \pm 0.01$ & $1.12 \pm 0.01$ & $1.03 \pm 0.03$ \\
\hline $\log (\mathrm{Ne} / \mathrm{O})$ & $-0.88 \pm 0.02$ & $-0.77 \pm 0.02$ & $-0.77 \pm 0.02$ & $-0.73 \pm 0.02$ \\
\hline $\mathrm{S}^{+} / \mathrm{H}^{+}\left(\times 10^{6}\right)$ & $0.03 \pm 0.00$ & $0.08 \pm 0.00$ & $0.11 \pm 0.00$ & $0.11 \pm 0.00$ \\
\hline $\mathrm{S}^{++} / \mathrm{H}^{+}\left(\times 10^{6}\right)$ & $0.20 \pm 0.01$ & $0.37 \pm 0.01$ & $0.54 \pm 0.04$ & $2.94 \pm 0.12$ \\
\hline ICF & $1.83 \pm 0.01$ & $1.24 \pm 0.02$ & $1.13 \pm 0.02$ & $1.81 \pm 0.05$ \\
\hline $\log (\mathrm{S} / \mathrm{O})$ & $-1.92 \pm 0.03$ & $-1.78 \pm 0.02$ & $-1.69 \pm 0.03$ & $-1.39 \pm 0.02$ \\
\hline $\mathrm{Cl}^{++} / \mathrm{H}^{+}\left(\times 10^{8}\right)$ & $\ldots$ & $0.36 \pm 0.01$ & $\ldots$ & $4.75 \pm 0.50$ \\
\hline ICF & $\ldots$ & $1.46 \pm 0.26$ & $\ldots$ & $1.57 \pm 0.19$ \\
\hline $\log (\mathrm{Cl} / \mathrm{O})$ & $\ldots$ & $-3.81 \pm 0.08$ & $\ldots$ & $-3.26 \pm 0.07$ \\
\hline $\mathrm{Ar}^{++} / \mathrm{H}^{+}\left(\times 10^{7}\right)$ & $\ldots$ & $0.90 \pm 0.02$ & $1.06 \pm 0.04$ & $7.06 \pm 0.18$ \\
\hline $\mathrm{Ar}^{+++} / \mathrm{H}^{+}\left(\times 10^{7}\right)$ & $1.01 \pm 0.03$ & $0.41 \pm 0.02$ & $0.38 \pm 0.09$ & $0.96 \pm 0.13$ \\
\hline ICF & $\ldots$ & $1.16 \pm 0.01$ & $1.10 \pm 0.01$ & $1.20 \pm 0.02$ \\
\hline $\log (\mathrm{Ar} / \mathrm{O})$ & $\ldots$ & $-2.52 \pm 0.02$ & $-2.49 \pm 0.04$ & $-2.20 \pm 0.02$ \\
\hline $\mathrm{Fe}^{++} / \mathrm{H}^{+}\left(\times 10^{6}\right)(4658)$ & $0.06 \pm 0.01$ & $0.08 \pm 0.01$ & $0.13 \pm 0.02$ & $\cdots$ \\
\hline $\mathrm{Fe}^{++} / \mathrm{H}^{+}\left(\times 10^{6}\right)(4988)$ & $0.09 \pm 0.01$ & $0.08 \pm 0.01$ & $0.24 \pm 0.02$ & $\ldots$ \\
\hline ICF & $17.74 \pm 1.02$ & $7.00 \pm 0.36$ & $5.05 \pm 0.35$ & $\ldots$ \\
\hline $\log (\mathrm{Fe} / \mathrm{O})(4658)$ & $-1.52 \pm 0.05$ & $-1.76 \pm 0.04$ & $-1.73 \pm 0.08$ & $\ldots$ \\
\hline $\log (\mathrm{Fe} / \mathrm{O})(4988)$ & $-1.31 \pm 0.04$ & $-1.75 \pm 0.04$ & $-1.47 \pm 0.05$ & $\ldots$ \\
\hline \multirow[b]{2}{*}{ Property } & \multicolumn{4}{|c|}{$\begin{array}{c}\text { GALAXY } \\
\text { (ESO PROGRAM) }\end{array}$} \\
\hline & NGC 6822V No.2 & NGC 6822X & TOL2138-405 No.1 & TOL2138-405 No.3 \\
\hline$T_{\mathrm{e}}(\mathrm{O}$ III) $(\mathrm{K})$ & $12218 \pm 150$ & $12385 \pm 385$ & $13931 \pm 122$ & $14453 \pm 211$ \\
\hline$T_{\mathrm{e}}\left(\mathrm{O}_{\text {II }}\right)(\mathrm{K})$ & $12020 \pm 168$ & $12159 \pm 434$ & $13292 \pm 141$ & $13609 \pm 245$ \\
\hline$T_{\mathrm{e}}(\mathrm{S}$ III) $(\mathrm{K})$ & $11406 \pm 124$ & $11623 \pm 320$ & $13491 \pm 101$ & $14063 \pm 175$ \\
\hline$N_{\mathrm{e}}(\mathrm{S}$ II $)\left(\mathrm{cm}^{-3}\right)$ & $10 \pm 10$ & $10 \pm 10$ & $373 \pm 53$ & $40 \pm 40$ \\
\hline $\mathrm{O}^{+} / \mathrm{H}^{+}\left(\times 10^{4}\right)$ & $0.18 \pm 0.01$ & $0.21 \pm 0.02$ & $0.18 \pm 0.01$ & $0.21 \pm 0.01$ \\
\hline $\mathrm{O}^{++} / \mathrm{H}^{+}\left(\times 10^{4}\right)$ & $1.10 \pm 0.04$ & $0.87 \pm 0.08$ & $0.86 \pm 0.02$ & $0.67 \pm 0.03$ \\
\hline $\mathrm{O}^{+++} / \mathrm{H}^{+}\left(\times 10^{6}\right)$ & $\ldots$ & $\ldots$ & $1.16 \pm 0.08$ & $2.72 \pm 0.29$ \\
\hline $\mathrm{O} / \mathrm{H}\left(\times 10^{4}\right)$ & $1.27 \pm 0.04$ & $1.07 \pm 0.08$ & $1.05 \pm 0.02$ & $0.91 \pm 0.03$ \\
\hline $12+\log (\mathrm{O} / \mathrm{H})$ & $8.10 \pm 0.01$ & $8.03 \pm 0.03$ & $8.02 \pm 0.01$ & $7.96 \pm 0.01$ \\
\hline $\mathrm{N}^{+} / \mathrm{H}^{+}\left(\times 10^{6}\right)$ & $0.47 \pm 0.03$ & $0.61 \pm 0.09$ & $1.19 \pm 0.05$ & $0.51 \pm 0.03$ \\
\hline ICF & $6.59 \pm 0.31$ & $5.05 \pm 0.56$ & $5.53 \pm 0.19$ & $4.32 \pm 0.22$ \\
\hline $\log (\mathrm{N} / \mathrm{O})$ & $-1.61 \pm 0.04$ & $-1.55 \pm 0.09$ & $-1.20 \pm 0.03$ & $-1.61 \pm 0.04$ \\
\hline $\mathrm{Ne}^{++} / \mathrm{H}^{+}\left(\times 10^{5}\right)$ & $2.44 \pm 0.11$ & $1.82 \pm 0.19$ & $1.88 \pm 0.06$ & $1.38 \pm 0.06$ \\
\hline ICF & $1.05 \pm 0.04$ & $1.11 \pm 0.08$ & $1.10 \pm 0.02$ & $1.16 \pm 0.03$ \\
\hline $\log (\mathrm{Ne} / \mathrm{O})$ & $-0.69 \pm 0.03$ & $-0.73 \pm 0.06$ & $-0.71 \pm 0.02$ & $-0.75 \pm 0.03$ \\
\hline $\mathrm{S}^{+} / \mathrm{H}^{+}\left(\times 10^{6}\right)$ & $0.14 \pm 0.00$ & $0.16 \pm 0.01$ & $0.25 \pm 0.01$ & $0.28 \pm 0.01$ \\
\hline
\end{tabular}


Table 8. continued.

\begin{tabular}{|c|c|c|c|c|}
\hline \multirow[b]{2}{*}{ Property } & \multicolumn{4}{|c|}{$\begin{array}{c}\text { GALAXY } \\
\text { (ESO PROGRAM) }\end{array}$} \\
\hline & $\begin{array}{c}\text { NGC 6822V No.2 } \\
(69 . \mathrm{C}-0203(\mathrm{~A}))\end{array}$ & $\begin{array}{c}\text { NGC 6822X } \\
(69 . C-0203(A))\end{array}$ & $\begin{array}{l}\text { TOL2138-405 No.1 } \\
(69 . \mathrm{C}-0203(\mathrm{~A}))\end{array}$ & $\begin{array}{c}\text { TOL2138-405 No.3 } \\
(69 . \mathrm{C}-0203(\mathrm{~A}))\end{array}$ \\
\hline $\mathrm{S}^{++} / \mathrm{H}^{+}\left(\times 10^{6}\right)$ & $2.54 \pm 0.16$ & $2.19 \pm 0.42$ & $1.37 \pm 0.05$ & $1.05 \pm 0.10$ \\
\hline ICF & $1.63 \pm 0.06$ & $1.34 \pm 0.09$ & $1.40 \pm 0.03$ & $1.23 \pm 0.03$ \\
\hline $\log (\mathrm{S} / \mathrm{O})$ & $-1.46 \pm 0.03$ & $-1.53 \pm 0.09$ & $-1.67 \pm 0.02$ & $-1.75 \pm 0.04$ \\
\hline $\mathrm{Cl}^{++} / \mathrm{H}^{+}\left(\times 10^{8}\right)$ & $4.27 \pm 0.66$ & $\ldots$ & $2.33 \pm 0.25$ & $\ldots$ \\
\hline ICF & $1.47 \pm 0.25$ & $\ldots$ & $1.38 \pm 0.19$ & $\ldots$ \\
\hline $\log (\mathrm{Cl} / \mathrm{O})$ & $-3.31 \pm 0.10$ & $\ldots$ & $-3.51 \pm 0.08$ & $\ldots$ \\
\hline $\mathrm{Ar}^{++} / \mathrm{H}^{+}\left(\times 10^{7}\right)$ & $6.28 \pm 0.21$ & $6.57 \pm 0.47$ & $\cdots$ & $\ldots$ \\
\hline $\mathrm{Ar}^{+++} / \mathrm{H}^{+}\left(\times 10^{7}\right)$ & $0.99 \pm 0.22$ & $\ldots$ & $1.26 \pm 0.07$ & $\ldots$ \\
\hline ICF & $1.47 \pm 0.25$ & $1.11 \pm 0.04$ & $\ldots$ & $\ldots$ \\
\hline $\log (\mathrm{Ar} / \mathrm{O})$ & $-2.24 \pm 0.03$ & $-2.17 \pm 0.05$ & $\ldots$ & $\ldots$ \\
\hline $\mathrm{Fe}^{++} / \mathrm{H}^{+}\left(\times 10^{6}\right)(4658)$ & $\ldots$ & $\ldots$ & $0.24 \pm 0.02$ & $0.29 \pm 0.05$ \\
\hline $\mathrm{Fe}^{++} / \mathrm{H}^{+}\left(\times 10^{6}\right)(4988)$ & $\ldots$ & $\ldots$ & $0.25 \pm 0.01$ & $0.45 \pm 0.05$ \\
\hline ICF & $\ldots$ & $\ldots$ & $7.61 \pm 0.29$ & $5.81 \pm 0.34$ \\
\hline $\log (\mathrm{Fe} / \mathrm{O})(4658)$ & $\ldots$ & $\ldots$ & $-1.76 \pm 0.04$ & $-1.74 \pm 0.08$ \\
\hline $\log (\mathrm{Fe} / \mathrm{O})(4988)$ & $\ldots$ & $\ldots$ & $-1.75 \pm 0.03$ & $-1.54 \pm 0.05$ \\
\hline \multirow{3}{*}{ Property } & \multicolumn{4}{|c|}{$\begin{array}{c}\text { GALAXY } \\
\text { (ESO PROGRAM) }\end{array}$} \\
\hline & TOL2146-391 No.1 & TOL2146-391 No.2 & TOL $2240-384$ & UM 160 No.1 \\
\hline & $(69 . \mathrm{C}-0203(\mathrm{~A}))$ & (69.C-0203(A)) & (69.C-0203(A)) & $(69 . C-0203(A))$ \\
\hline 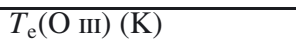 & $15810 \pm 151$ & $16050 \pm 164$ & $14539 \pm 132$ & $14096 \pm 150$ \\
\hline$T_{\mathrm{e}}\left(\mathrm{O}_{\text {II }}\right)(\mathrm{K})$ & $14277 \pm 179$ & $14371 \pm 195$ & $13658 \pm 153$ & $13396 \pm 173$ \\
\hline$T_{\mathrm{e}}(\mathrm{S}$ III $)(\mathrm{K})$ & $15410 \pm 125$ & $15627 \pm 136$ & $14154 \pm 109$ & $13675 \pm 124$ \\
\hline$N_{\mathrm{e}}\left(\mathrm{S}_{\mathrm{II}}\right)\left(\mathrm{cm}^{-3}\right)$ & $162 \pm 42$ & $147 \pm 46$ & $344 \pm 69$ & $142 \pm 45$ \\
\hline $\mathrm{O}^{+} / \mathrm{H}^{+}\left(\times 10^{4}\right)$ & $0.08 \pm 0.00$ & $0.08 \pm 0.00$ & $0.06 \pm 0.00$ & $0.16 \pm 0.01$ \\
\hline $\mathrm{O}^{++} / \mathrm{H}^{+}\left(\times 10^{4}\right)$ & $0.57 \pm 0.02$ & $0.53 \pm 0.01$ & $0.79 \pm 0.02$ & $0.70 \pm 0.02$ \\
\hline $\mathrm{O}^{+++} / \mathrm{H}^{+}\left(\times 10^{6}\right)$ & $1.15 \pm 0.05$ & $1.43 \pm 0.08$ & $1.27 \pm 0.07$ & $0.32 \pm 0.05$ \\
\hline $\mathrm{O} / \mathrm{H}\left(\times 10^{4}\right)$ & $0.66 \pm 0.02$ & $0.62 \pm 0.02$ & $0.86 \pm 0.02$ & $0.86 \pm 0.02$ \\
\hline $12+\log (\mathrm{O} / \mathrm{H})$ & $7.82 \pm 0.01$ & $7.79 \pm 0.01$ & $7.93 \pm 0.01$ & $7.94 \pm 0.01$ \\
\hline $\mathrm{N}^{+} / \mathrm{H}^{+}\left(\times 10^{6}\right)$ & $0.24 \pm 0.01$ & $0.19 \pm 0.01$ & $0.51 \pm 0.02$ & $0.38 \pm 0.02$ \\
\hline ICF & $7.59 \pm 0.30$ & $7.56 \pm 0.32$ & $13.16 \pm 0.53$ & $5.06 \pm 0.20$ \\
\hline $\log (\mathrm{N} / \mathrm{O})$ & $-1.57 \pm 0.03$ & $-1.63 \pm 0.03$ & $-1.11 \pm 0.03$ & $-1.65 \pm 0.03$ \\
\hline $\mathrm{Ne}^{++} / \mathrm{H}^{+}\left(\times 10^{5}\right)$ & $1.03 \pm 0.03$ & $0.98 \pm 0.03$ & $0.96 \pm 0.03$ & $1.25 \pm 0.04$ \\
\hline ICF & $1.06 \pm 0.02$ & $1.06 \pm 0.02$ & $1.01 \pm 0.02$ & $1.10 \pm 0.02$ \\
\hline $\log (\mathrm{Ne} / \mathrm{O})$ & $-0.78 \pm 0.02$ & $-0.77 \pm 0.02$ & $-0.95 \pm 0.02$ & $-0.80 \pm 0.02$ \\
\hline $\mathrm{S}^{+} / \mathrm{H}^{+}\left(\times 10^{6}\right)$ & $0.13 \pm 0.00$ & $0.11 \pm 0.00$ & $0.09 \pm 0.00$ & $0.20 \pm 0.00$ \\
\hline $\mathrm{S}^{++} / \mathrm{H}^{+}\left(\times 10^{6}\right)$ & $0.72 \pm 0.02$ & $0.66 \pm 0.03$ & $0.54 \pm 0.03$ & $1.24 \pm 0.05$ \\
\hline ICF & $1.50 \pm 0.03$ & $1.47 \pm 0.03$ & $2.26 \pm 0.07$ & $1.30 \pm 0.03$ \\
\hline $\log (\mathrm{S} / \mathrm{O})$ & $-1.72 \pm 0.02$ & $-1.74 \pm 0.02$ & $-1.78 \pm 0.03$ & $-1.66 \pm 0.02$ \\
\hline $\mathrm{Cl}^{++} / \mathrm{H}^{+}\left(\times 10^{8}\right)$ & $0.96 \pm 0.10$ & $0.87 \pm 0.13$ & $1.67 \pm 0.13$ & $2.07 \pm 0.22$ \\
\hline ICF & $1.74 \pm 0.37$ & $1.74 \pm 0.40$ & $2.53 \pm 0.60$ & $1.37 \pm 0.23$ \\
\hline $\log (\mathrm{Cl} / \mathrm{O})$ & $-3.60 \pm 0.10$ & $-3.61 \pm 0.12$ & $-3.30 \pm 0.11$ & $-3.48 \pm 0.09$ \\
\hline $\mathrm{Ar}^{++} / \mathrm{H}^{+}\left(\times 10^{7}\right)$ & $1.89 \pm 0.04$ & $1.62 \pm 0.04$ & $\cdots$ & $2.63 \pm 0.07$ \\
\hline $\mathrm{Ar}^{+++} / \mathrm{H}^{+}\left(\times 10^{7}\right)$ & $1.06 \pm 0.05$ & $0.97 \pm 0.06$ & $1.54 \pm 0.07$ & $0.56 \pm 0.08$ \\
\hline ICF & $1.26 \pm 0.02$ & $1.27 \pm 0.02$ & $\ldots$ & $1.13 \pm 0.01$ \\
\hline $\log (\mathrm{Ar} / \mathrm{O})$ & $-2.44 \pm 0.02$ & $-2.48 \pm 0.02$ & $\ldots$ & $-2.46 \pm 0.02$ \\
\hline $\mathrm{Fe}^{++} / \mathrm{H}^{+}\left(\times 10^{6}\right)(4658)$ & $0.13 \pm 0.01$ & $0.11 \pm 0.01$ & $0.15 \pm 0.01$ & $0.17 \pm 0.02$ \\
\hline $\mathrm{Fe}^{++} / \mathrm{H}^{+}\left(\times 10^{6}\right)(4988)$ & $0.20 \pm 0.01$ & $0.20 \pm 0.01$ & $0.18 \pm 0.02$ & $0.19 \pm 0.02$ \\
\hline ICF & $10.89 \pm 0.48$ & $10.85 \pm 0.51$ & $19.53 \pm 0.84$ & $6.94 \pm 0.32$ \\
\hline $\log (\mathrm{Fe} / \mathrm{O})(4658)$ & $-1.66 \pm 0.04$ & $-1.70 \pm 0.06$ & $-1.46 \pm 0.04$ & $-1.85 \pm 0.05$ \\
\hline $\log (\mathrm{Fe} / \mathrm{O})(4988)$ & $-1.49 \pm 0.03$ & $-1.45 \pm 0.04$ & $-1.38 \pm 0.04$ & $-1.82 \pm 0.05$ \\
\hline
\end{tabular}


Table 8. continued.

\begin{tabular}{|c|c|c|}
\hline \multirow[b]{2}{*}{ Property } & \multicolumn{2}{|c|}{$\begin{array}{c}\text { GALAXY } \\
\text { (ESO PROGRAM) }\end{array}$} \\
\hline & $\begin{array}{c}\text { UM } 160 \text { No.2 } \\
(69 . \mathrm{C}-0203(\mathrm{~A}))\end{array}$ & $\begin{array}{c}\text { UM } 160 \text { No.3 } \\
(69 . \mathrm{C}-0203(\mathrm{~A}))\end{array}$ \\
\hline$\overline{T_{\mathrm{e}}(\mathrm{O} \text { III })(\mathrm{K})}$ & $12392 \pm 226$ & $11689 \pm 84$ \\
\hline$T_{\mathrm{e}}\left(\mathrm{O}_{\text {II }}\right)(\mathrm{K})$ & $12165 \pm 255$ & $12037 \pm 93$ \\
\hline$T_{\mathrm{e}}(\mathrm{S}$ III $)(\mathrm{K})$ & $11633 \pm 187$ & $11658 \pm 69$ \\
\hline$N_{\mathrm{e}}(\mathrm{S}$ II $)\left(\mathrm{cm}^{-3}\right)$ & $10 \pm 10$ & $11 \pm 11$ \\
\hline $\mathrm{O}^{+} / \mathrm{H}^{+}\left(\times 10^{4}\right)$ & $0.27 \pm 0.02$ & $0.34 \pm 0.01$ \\
\hline $\mathrm{O}^{++} / \mathrm{H}^{+}\left(\times 10^{4}\right)$ & $0.95 \pm 0.05$ & $0.92 \pm 0.02$ \\
\hline $\mathrm{O}^{+++} / \mathrm{H}^{+}\left(\times 10^{6}\right)$ & $1.18 \pm 0.25$ & $\ldots$ \\
\hline $\mathrm{O} / \mathrm{H}\left(\times 10^{4}\right)$ & $1.24 \pm 0.06$ & $1.25 \pm 0.03$ \\
\hline $12+\log (\mathrm{O} / \mathrm{H})$ & $8.09 \pm 0.02$ & $8.10 \pm 0.01$ \\
\hline $\mathrm{N}^{+} / \mathrm{H}^{+}\left(\times 10^{6}\right)$ & $0.69 \pm 0.05$ & $1.04 \pm 0.04$ \\
\hline ICF & $4.55 \pm 0.28$ & $3.83 \pm 0.10$ \\
\hline $\log (\mathrm{N} / \mathrm{O})$ & $-1.59 \pm 0.05$ & $-1.50 \pm 0.02$ \\
\hline $\mathrm{Ne}^{++} / \mathrm{H}^{+}\left(\times 10^{5}\right)$ & $1.97 \pm 0.12$ & $1.86 \pm 0.06$ \\
\hline ICF & $1.15 \pm 0.05$ & $1.21 \pm 0.02$ \\
\hline $\log (\mathrm{Ne} / \mathrm{O})$ & $-0.74 \pm 0.04$ & $-0.75 \pm 0.02$ \\
\hline $\mathrm{S}^{+} / \mathrm{H}^{+}\left(\times 10^{6}\right)$ & $0.31 \pm 0.01$ & $0.44 \pm 0.01$ \\
\hline $\mathrm{S}^{++} / \mathrm{H}^{+}\left(\times 10^{6}\right)$ & $2.28 \pm 0.20$ & $2.37 \pm 0.17$ \\
\hline $\mathrm{ICF}$ & $1.30 \pm 0.05$ & $1.20 \pm 0.02$ \\
\hline $\log (\mathrm{S} / \mathrm{O})$ & $-1.57 \pm 0.04$ & $-1.57 \pm 0.03$ \\
\hline $\mathrm{Ar}^{++} / \mathrm{H}^{+}\left(\times 10^{7}\right)$ & $3.70 \pm 0.19$ & $4.69 \pm 0.14$ \\
\hline $\mathrm{Ar}^{+++} / \mathrm{H}^{+}\left(\times 10^{7}\right)$ & 等 & \\
\hline $\mathrm{ICF}$ & $1.09 \pm 0.02$ & $1.07 \pm 0.01$ \\
\hline $\log (\mathrm{Ar} / \mathrm{O})$ & $-2.49 \pm 0.03$ & $-2.40 \pm 0.02$ \\
\hline $\mathrm{Fe}^{++} / \mathrm{H}^{+}\left(\times 10^{6}\right)(4988)$ & $0.19 \pm 0.05$ & $0.33 \pm 0.05$ \\
\hline $\mathrm{ICF}$ & $6.10 \pm 0.44$ & $5.05 \pm 0.16$ \\
\hline $\log (\mathrm{Fe} / \mathrm{O})(4988)$ & $-2.03 \pm 0.12$ & $-1.87 \pm 0.07$ \\
\hline
\end{tabular}

Table 10. Electron temperatures and electron number densities derived from emission line flux ratios.

\begin{tabular}{|c|c|c|c|c|c|}
\hline Name & $t_{\mathrm{e}}(\mathrm{O} \text { III })^{a}$ & $t_{\mathrm{e}}\left(\mathrm{S}_{\mathrm{III}}\right)^{a}$ & $t_{\mathrm{e}}(\mathrm{N} \mathrm{II})^{a}$ & $t_{\mathrm{e}}(\mathrm{O} \text { II })^{a}$ & $t_{\mathrm{e}}\left(\mathrm{S}_{\mathrm{II}}\right)^{a}$ \\
\hline UM 283D $\mathrm{D}^{e}$ & $1.2360 \pm 0.0338$ & $1.1116 \pm 0.0603$ & $\ldots$ & $1.1827 \pm 0.0251$ & $0.8560 \pm 0.0350$ \\
\hline NGC 346 D No. $1^{g}$ & $1.3253 \pm 0.0126$ & $\ldots$ & $\ldots$ & $\ldots$ & $0.8326 \pm 0.0041$ \\
\hline NGC 346 D No. $2^{g}$ & $1.3332 \pm 0.0123$ & $\ldots$ & $\ldots$ & $\ldots$ & $0.9519 \pm 0.0148$ \\
\hline NGC 346 D No. $3^{g}$ & $1.2741 \pm 0.0116$ & $\ldots$ & $\ldots$ & $\ldots$ & $0.8714 \pm 0.0085$ \\
\hline NGC 346 D No. $4^{g}$ & $1.3036 \pm 0.0121$ & $\ldots$ & $\ldots$ & $\ldots$ & $0.8445 \pm 0.0141$ \\
\hline NGC 456 A No. $1^{g}$ & $1.2308 \pm 0.0108$ & $\ldots$ & $1.1277 \pm 0.0088$ & $1.2928 \pm 0.0037$ & $0.9408 \pm 0.0202$ \\
\hline NGC 456 A No. $3^{g}$ & $1.2134 \pm 0.0117$ & $\ldots$ & $\ldots$ & $\ldots$ & $0.9413 \pm 0.0616$ \\
\hline NGC 456 A No. $1^{f}$ & $1.2281 \pm 0.0088$ & $\ldots$ & $1.1588 \pm 0.0310$ & $1.1708 \pm 0.0011$ & $0.8290 \pm 0.0036$ \\
\hline NGC 456 A No. $2^{f}$ & $1.2029 \pm 0.0094$ & $\ldots$ & $1.0650 \pm 0.1009$ & $1.2554 \pm 0.0034$ & $0.7738 \pm 0.0170$ \\
\hline NGC 456 A No. $3^{f}$ & $1.2084 \pm 0.0088$ & $\ldots$ & $1.1750 \pm 0.0924$ & $1.1371 \pm 0.0012$ & $0.9413 \pm 0.0616$ \\
\hline $\mathrm{UM} 133 \mathrm{H}^{e}$ & $1.5504 \pm 0.0243$ & 1.9303: & $\ldots$ & $1.4161 \pm 0.0219$ & \\
\hline $\mathrm{UM} 133 \mathrm{O}^{e}$ & $1.5415 \pm 0.0177$ & $\ldots$ & $\ldots$ & $\ldots$ & $0.7783 \pm 0.0474$ \\
\hline UM $382^{e}$ & $1.5616 \pm 0.0141$ & $\ldots$ & $\ldots$ & $\ldots$ & $1.0093 \pm 0.1105$ \\
\hline $\mathrm{UM} 408^{e}$ & $1.3655 \pm 0.0147$ & $\ldots$ & $\ldots$ & $\ldots$ & $0.8054 \pm 0.0527$ \\
\hline $\mathrm{UM} 417^{e}$ & $1.7086 \pm 0.0177$ & $\ldots$ & $\ldots$ & $\ldots$ & $0.7872 \pm 0.0512$ \\
\hline UM 420 No. $1^{f}$ & $1.2149 \pm 0.0386$ & $\ldots$ & $\ldots$ & & $0.8681 \pm 0.0457$ \\
\hline CAM $0357-3915^{g}$ & $1.5401 \pm 0.0193$ & $\ldots$ & $\ldots$ & $1.1744 \pm 0.0527$ & $\ldots$ \\
\hline CAM $0357-3915^{f}$ & $1.5124 \pm 0.0148$ & $\ldots$ & $\ldots$ & $\ldots$ & $0.8712 \pm 0.0475$ \\
\hline Tol $0513-393^{g}$ & $1.5160 \pm 0.0144$ & $\ldots$ & $\ldots$ & $1.6405 \pm 0.0329$ & $1.0647 \pm 0.0617$ \\
\hline Tol $0513-393^{f}$ & $1.5131 \pm 0.0136$ & $\ldots$ & $\ldots$ & $\ldots$ & $0.7204 \pm 0.0229$ \\
\hline HE $2-10 C^{e}$ & $1.2415 \pm 0.0218$ & 0.7002: & $0.7787 \pm 0.0136$ & $0.8394 \pm 0.0009$ & $0.5250 \pm 0.0091$ \\
\hline HE $2-10 \mathrm{E}^{e}$ & $0.7450 \pm 0.0355$ & $0.6980 \pm 0.0198$ & $\ldots$ & $0.8235 \pm 0.0002$ & $0.4945 \pm 0.0045$ \\
\hline NGC $3125^{e}$ & $1.1087 \pm 0.0071$ & $0.9679 \pm 0.0112$ & $\ldots$ & $1.6763 \pm 0.0021$ & $0.5907 \pm 0.0024$ \\
\hline Mrk $1259^{e}$ & $1.0126 \pm 0.0340$ & $0.7514 \pm 0.0230$ & $0.7733 \pm 0.0296$ & $1.0665 \pm 0.0021$ & $0.6194 \pm 0.0157$ \\
\hline $\operatorname{Mrk} 1271^{e}$ & $1.3428 \pm 0.0102$ & $1.2834 \pm 0.0172$ & $\ldots$ & $1.2282 \pm 0.0022$ & $0.7088 \pm 0.0040$ \\
\hline
\end{tabular}


Table 10. continued.

\begin{tabular}{|c|c|c|c|c|c|}
\hline Name & $t_{\mathrm{e}}(\mathrm{O} \text { III })^{a}$ & $t_{\mathrm{e}}\left(\mathrm{S}_{\mathrm{III}}\right)^{a}$ & $t_{\mathrm{e}}\left(\mathrm{N}_{\mathrm{II}}\right)^{a}$ & $t_{\mathrm{e}}\left(\mathrm{O}_{\mathrm{II}}\right)^{a}$ & $t_{\mathrm{e}}\left(\mathrm{S}_{\mathrm{II}}\right)^{a}$ \\
\hline POX $4^{e}$ & $1.3089 \pm 0.0097$ & $1.3989 \pm 0.0278$ & $\ldots$ & $1.2326 \pm 0.0016$ & $0.7360 \pm 0.0044$ \\
\hline Tol 1214-277 & $2.0020 \pm 0.0226$ & 2.4681: & $\ldots$ & $\ldots$ & $\ldots$ \\
\hline Tol 1214-277 ${ }^{f}$ & $1.9675 \pm 0.0224$ & $\ldots$ & $\ldots$ & $\ldots$ & $1.3357 \pm 0.1427$ \\
\hline Tol 65 No. $1^{f}$ & $1.7844 \pm 0.0189$ & $\ldots$ & $\ldots$ & $\ldots$ & $1.0002 \pm 0.0476$ \\
\hline Tol 65 No. $2^{f}$ & $1.7552 \pm 0.0261$ & $\ldots$ & $\ldots$ & $\ldots$ & $1.5364 \pm 0.1958$ \\
\hline $\mathrm{J} 1253-0312^{e}$ & $1.3851 \pm 0.0108$ & $1.2433 \pm 0.0314$ & $1.2040 \pm 0.0201$ & $0.9428 \pm 0.0013$ & $0.7115 \pm 0.0010$ \\
\hline NGC $5253 \mathrm{No.C}^{e}$ & $1.2279 \pm 0.0085$ & $1.2124 \pm 0.0278$ & $1.1129 \pm 0.0135$ & $1.2910 \pm 0.0010$ & $0.8477 \pm 0.0037$ \\
\hline NGC 5253 No.C $2^{e}$ & $1.0267 \pm 0.0065$ & $1.1402 \pm 0.0267$ & $1.0788 \pm 0.0394$ & $1.3713 \pm 0.0015$ & $0.6389 \pm 0.0058$ \\
\hline NGC 5253 No.P1 $^{e}$ & $1.3531 \pm 0.0056$ & $1.1159 \pm 0.0338$ & $\ldots$ & $1.0166 \pm 0.0002$ & $0.7937 \pm 0.0107$ \\
\hline NGC 5253 No.P2 $^{e}$ & $1.2412 \pm 0.0087$ & $1.2595 \pm 0.0302$ & $1.1062 \pm 0.0190$ & $1.1152 \pm 0.0001$ & $0.8471 \pm 0.0062$ \\
\hline Tol 89 No. $1^{e}$ & $1.0263 \pm 0.0074$ & $0.9909 \pm 0.0204$ & $1.0333 \pm 0.0376$ & $1.1099 \pm 0.0010$ & $0.6952 \pm 0.0054$ \\
\hline Tol 89 No. $2^{e}$ & $0.9377 \pm 0.0160$ & $0.7122 \pm 0.0300$ & $\ldots$ & (1) & $\ldots$ \\
\hline NGC 5408 No. $^{e}$ & $1.5585 \pm 0.0132$ & $1.7851 \pm 0.0531$ & $1.3274 \pm 0.0103$ & $1.0603 \pm 0.0006$ & $0.8777 \pm 0.0092$ \\
\hline NGC 5408 No. $2^{e}$ & $1.3667 \pm 0.0107$ & $1.6806 \pm 0.0484$ & $1.6307 \pm 0.0979$ & $1.0596 \pm 0.0006$ & $0.6698 \pm 0.0031$ \\
\hline Tol $1457-262^{e}$ & $1.1892 \pm 0.0081$ & $1.2465 \pm 0.0320$ & $0.9042 \pm 0.0330$ & $0.8475 \pm 0.0007$ & $1.0700 \pm 0.0088$ \\
\hline Tol 1924-416 No. $1^{e}$ & $1.2485 \pm 0.0096$ & $1.2018 \pm 0.0575$ & $\ldots$ & $1.2766 \pm 0.0052$ & $0.6030 \pm 0.0091$ \\
\hline Tol 1924-416 No. $2^{e}$ & $1.2269 \pm 0.0086$ & $1.3316 \pm 0.0331$ & $\ldots$ & $1.3324 \pm 0.0008$ & $0.6418 \pm 0.0027$ \\
\hline NGC $6822 \mathrm{~V}^{e}$ & $1.1429 \pm 0.0071$ & $1.1219 \pm 0.0230$ & $1.1547 \pm 0.0539$ & $1.5430 \pm 0.0022$ & $0.6785 \pm 0.0017$ \\
\hline NGC 6822 V No. $1^{g}$ & $1.1686 \pm 0.0135$ & $\ldots$ & $\ldots$ & $1.1566 \pm 0.0145$ & $1.1624 \pm 0.2175$ \\
\hline NGC $6822 \mathrm{~V}$ No. $2^{g}$ & $1.2051 \pm 0.0188$ & $\ldots$ & $\ldots$ & $1.1680 \pm 0.0224$ & $\ldots$ \\
\hline NGC 6822 V No. $1^{f}$ & $1.1924 \pm 0.0104$ & $\ldots$ & $\ldots$ & $1.3069 \pm 0.0229$ & $0.9634 \pm 0.0955$ \\
\hline NGC $6822 \mathrm{~V}$ No. $2^{f}$ & $1.2218 \pm 0.0150$ & $\ldots$ & $\ldots$ & $1.2393 \pm 0.0553$ & $\ldots$ \\
\hline Tol 2138-405 No. $1^{g}$ & $1.3129 \pm 0.0154$ & $\ldots$ & $\ldots$ & $1.2188 \pm 0.0287$ & $\ldots$ \\
\hline Tol 2138-405 No. $1^{f}$ & $1.3931 \pm 0.0122$ & $\ldots$ & $1.1366 \pm 0.0959$ & $\ldots$ & $0.8475 \pm 0.0192$ \\
\hline Tol 2138-405 No. $3^{f}$ & $1.4453 \pm 0.0211$ & $\ldots$ & $5.8785 \pm 8.0959$ & $\ldots$ & $1.0108 \pm 0.0709$ \\
\hline Tol 2146-391 No. $1^{g}$ & $1.6005 \pm 0.0198$ & $\ldots$ & $\ldots$ & $1.2592 \pm 0.0379$ & $1.5103 \pm 0.2097$ \\
\hline Tol 2146-391 No. $1^{f}$ & $1.5810 \pm 0.0151$ & $\ldots$ & $\ldots$ & $\ldots$ & $0.8889 \pm 0.0285$ \\
\hline Tol 2146-391 No. $2^{f}$ & $1.6050 \pm 0.0164$ & $\ldots$ & $\ldots$ & $\ldots$ & $0.9371 \pm 0.0613$ \\
\hline Tol 2240-384 & $1.5777 \pm 0.0198$ & $\ldots$ & $3.5398 \pm 0.8459$ & $\ldots$ & $1.1984 \pm 0.2105$ \\
\hline Tol 2240-384 & $1.4539 \pm 0.0132$ & $\ldots$ & $2.3467 \pm 0.2932$ & $\ldots$ & $1.0445 \pm 0.0480$ \\
\hline PHL 293B ${ }^{e}$ & $1.6926 \pm 0.0190$ & $\ldots$ & $\ldots$ & $\ldots$ & $0.9582 \pm 0.1079$ \\
\hline UM 160 No. $1^{g}$ & $1.3613 \pm 0.0271$ & $\ldots$ & $\ldots$ & $1.3727 \pm 0.0375$ & $\ldots$ \\
\hline UM 160 No. $1^{f}$ & $1.4096 \pm 0.0150$ & $\ldots$ & $\ldots$ & $1.1803 \pm 0.0152$ & $0.8529 \pm 0.0429$ \\
\hline UM 160 No. $2^{f}$ & $1.2392 \pm 0.0226$ & $\ldots$ & $\ldots$ & $1.1756 \pm 0.0562$ & $\ldots$ \\
\hline UM 160 No. $3^{f}$ & $1.1689 \pm 0.0084$ & $\ldots$ & $\ldots$ & $1.3100 \pm 0.0342$ & $0.9275 \pm 0.0693$ \\
\hline SBS0335-052 $\mathrm{E}^{b}$ & $2.0487 \pm 0.0240$ & $1.8704 \pm 0.0804$ & $\ldots$ & $\ldots$ & $\ldots$ \\
\hline $\mathrm{J} 0519+0007^{c}$ & $2.0143 \pm 0.0238$ & $\ldots$ & $1.7995 \pm 0.1746$ & $\ldots$ & $\ldots$ \\
\hline Name & $N_{\mathrm{e}}\left(\mathrm{S}_{\mathrm{II}}\right)^{d}$ & $N_{\mathrm{e}}\left(\mathrm{O}_{\text {II }}\right)^{d}$ & $N_{\mathrm{e}}\left(\mathrm{Cl}_{\mathrm{III}}\right)^{d}$ & $N_{\mathrm{e}}(\mathrm{Ar} \mathrm{IV})^{d}$ & \\
\hline $\mathrm{UM}^{283 \mathrm{D}^{e}}$ & $15 \pm 15$ & $43 \pm 11$ & & $\ldots$ & \\
\hline NGC346 A No. $2^{f}$ & $42 \pm 32$ & $25 \pm 12$ & $1046 \pm 823$ & $\ldots$ & \\
\hline NGC346 A No. $3^{f}$ & $51 \pm 37$ & $70 \pm 14$ & $3043 \pm 1845$ & $\ldots$ & \\
\hline NGC346 B No. $1^{f}$ & $65 \pm 35$ & $48 \pm 15$ & $\ldots$ & $\ldots$ & \\
\hline NGC346 D No. $1^{f}$ & $13 \pm 12$ & $39 \pm 13$ & $2227 \pm 357$ & $\ldots$ & \\
\hline NGC346 D No. $2^{f}$ & $12 \pm 11$ & $48 \pm 16$ & $2496 \pm 742$ & $\ldots$ & \\
\hline NGC346 D No. $3^{f}$ & $146 \pm 39$ & $110 \pm 16$ & $1999 \pm 712$ & $\ldots$ & \\
\hline NGC346 D No. $4^{f}$ & $105 \pm 36$ & $70 \pm 14$ & $2217 \pm 753$ & $\ldots$ & \\
\hline NGC456 A No. $1^{f}$ & $387 \pm 51$ & $233 \pm 18$ & $1179 \pm 359$ & $\ldots$ & \\
\hline NGC456 A No. $2^{f}$ & $87 \pm 36$ & $86 \pm 15$ & $894 \pm 752$ & $\ldots$ & \\
\hline NGC456 A No. $3^{f}$ & $124 \pm 36$ & $113 \pm 16$ & $450 \pm 449$ & $\ldots$ & \\
\hline NGC456 A No. $3^{g}$ & $114 \pm 44$ & $\ldots$ & $2153 \pm 1794$ & $\ldots$ & \\
\hline $\mathrm{UM} 133 \mathrm{H}^{e}$ & $59 \pm 40$ & $42 \pm 15$ & $\ldots$ & $\ldots$ & \\
\hline $\mathrm{UM} 133 \mathrm{O}^{e}$ & $34 \pm 33$ & $38 \pm 11$ & $\ldots$ & $\ldots$ & \\
\hline $\mathrm{UM} 417^{e}$ & $83 \pm 42$ & $62 \pm 14$ & $\ldots$ & $\ldots$ & \\
\hline UM 420 No. $1^{f}$ & $49 \pm 47$ & $91 \pm 16$ & $\ldots$ & $\ldots$ & \\
\hline UM 420 No. $2^{f}$ & $60 \pm 45$ & $73 \pm 18$ & $\ldots$ & $\ldots$ & \\
\hline Mrk $600^{e}$ & $83 \pm 45$ & $302 \pm 24$ & $52 \pm 51$ & $\ldots$ & \\
\hline CAM 0357-3915 & $216 \pm 54$ & $205 \pm 19$ & $250 \pm 248$ & $\ldots$ & \\
\hline CAM 0357-3915 & $254 \pm 76$ & $\ldots$ & $3824 \pm 3824$ & $410 \pm 409$ & \\
\hline Tol $0513-393^{f}$ & $273 \pm 54$ & $198 \pm 19$ & $\ldots$ & $\ldots$ & \\
\hline He $2-10 C^{e}$ & $1551 \pm 143$ & $1449 \pm 17$ & $176 \pm 175$ & $\ldots$ & \\
\hline He $2-10 \mathrm{E}^{e}$ & $1331 \pm 134$ & $1187 \pm 15$ & $7513 \pm 2287$ & $\ldots$ & \\
\hline NGC $3125^{e}$ & $276 \pm 48$ & $265 \pm 11$ & $135 \pm 135$ & $\ldots$ & \\
\hline Mrk $1259^{e}$ & $1333 \pm 130$ & $3061 \pm 42$ & $\ldots$ & $\ldots$ & \\
\hline Mrk $1271^{e}$ & $87 \pm 36$ & $202 \pm 8$ & $\ldots$ & $\ldots$ & \\
\hline $\operatorname{Pox} 4^{e}$ & $151 \pm 39$ & $128 \pm 9$ & $\ldots$ & $\ldots$ & \\
\hline
\end{tabular}


Table 10. continued.

\begin{tabular}{|c|c|c|c|c|}
\hline$\overline{\text { Name }}$ & $N_{\mathrm{e}}\left(\mathrm{S}_{\mathrm{II}}\right)^{d}$ & $N_{\mathrm{e}}(\mathrm{O} \text { II })^{d}$ & $N_{\mathrm{e}}(\mathrm{Cl} \text { III })^{d}$ & $N_{\mathrm{e}}(\mathrm{Ar} \mathrm{IV})^{d}$ \\
\hline Tol 65 No. $2^{f}$ & $100 \pm 50$ & & $\ldots$ & $1112 \pm 1111$ \\
\hline $\mathrm{J} 1253-0312^{e}$ & $1726 \pm 153$ & $230 \pm 12$ & $\ldots$ & $\ldots$ \\
\hline 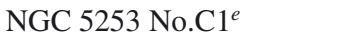 & $723 \pm 69$ & $458 \pm 13$ & $1139 \pm 369$ & $\ldots$ \\
\hline NGC 5253 No.C2 $2^{e}$ & $271 \pm 47$ & $252 \pm 11$ & $947 \pm 552$ & $\ldots$ \\
\hline NGC 5253 No.P1 ${ }^{e}$ & $366 \pm 50$ & $394 \pm 15$ & $3289 \pm 1889$ & $\ldots$ \\
\hline NGC 5253 No.P2 ${ }^{e}$ & $1108 \pm 99$ & $904 \pm 17$ & $2503 \pm 955$ & $\ldots$ \\
\hline Tol 89 No. $1^{e}$ & $147 \pm 38$ & $139 \pm 12$ & $178 \pm 158$ & $\ldots$ \\
\hline Tol 89 No. $2^{e}$ & $149 \pm 52$ & $91 \pm 14$ & $\ldots$ & $\ldots$ \\
\hline NGC 5408 No. $1^{e}$ & $308 \pm 46$ & $282 \pm 8$ & $863 \pm 862$ & $\ldots$ \\
\hline NGC 5408 No. $2^{e}$ & $133 \pm 36$ & $106 \pm 9$ & $\ldots$ & $\ldots$ \\
\hline Tol $1457-262^{e}$ & $104 \pm 35$ & $277 \pm 16$ & $437 \pm 437$ & $\ldots$ \\
\hline Tol 1924-416 No. $1^{e}$ & $126 \pm 37$ & $112 \pm 13$ & $\ldots$ & $\ldots$ \\
\hline Tol 1924-416 No. $2^{e}$ & $178 \pm 41$ & $117 \pm 14$ & $\ldots$ & $\ldots$ \\
\hline NGC $6822 \mathrm{~V}^{e}$ & $133 \pm 37$ & $102 \pm 11$ & $\ldots$ & $\ldots$ \\
\hline NGC6822V No. $1^{f}$ & $83 \pm 54$ & $117 \pm 19$ & $491 \pm 490$ & $\ldots$ \\
\hline NGC6822V No. $1^{g}$ & $86 \pm 48$ & $\ldots$ & $2053 \pm 2052$ & $\ldots$ \\
\hline Tol 2138-405 No. $1^{f}$ & $373 \pm 53$ & $273 \pm 21$ & $\ldots$ & $\ldots$ \\
\hline Tol 2138-405 No. $2^{f}$ & $172 \pm 43$ & $126 \pm 16$ & $996 \pm 995$ & $\ldots$ \\
\hline Tol 2138-405 No. $3^{f}$ & $40 \pm 40$ & $119 \pm 20$ & $\ldots$ & $\ldots$ \\
\hline Tol 2146-391 No. $1^{f}$ & $162 \pm 42$ & $59 \pm 14$ & $\ldots$ & $\ldots$ \\
\hline Tol 2146-391 No. $2^{f}$ & $147 \pm 46$ & $18 \pm 15$ & $\ldots$ & $\ldots$ \\
\hline Tol 2240-384 ${ }^{f}$ & $344 \pm 69$ & $100 \pm 50$ & $\ldots$ & $\ldots$ \\
\hline PHL 293B ${ }^{e}$ & $41 \pm 41$ & $109 \pm 16$ & $\ldots$ & $\ldots$ \\
\hline UM 160 No. $1^{f}$ & $142 \pm 45$ & $96 \pm 19$ & $1311 \pm 1310$ & $\ldots$ \\
\hline UM 160 No. $3^{f}$ & $11 \pm 11$ & $140 \pm 24$ & $2136 \pm 2135$ & $\ldots$ \\
\hline SBS0335-052E No. $1^{b}$ & $331 \pm 60$ & $338 \pm 26$ & $264 \pm 263$ & $\ldots$ \\
\hline SBS0335-052E No. $2^{b}$ & $91 \pm 89$ & $98 \pm 40$ & $\ldots$ & $\ldots$ \\
\hline SBS 0335-052E No. $4+5^{b}$ & $228 \pm 81$ & $119 \pm 19$ & $\ldots$ & $\ldots$ \\
\hline SBS0335-052 $W^{b}$ & $505 \pm 503$ & $107 \pm 96$ & $\ldots$ & $\ldots$ \\
\hline
\end{tabular}

Notes. ${ }^{(a)} t_{\mathrm{e}}=10^{-4} T_{\mathrm{e}}$; ${ }^{(b)}$ Izotov et al. (2009); ${ }^{(c)}$ Guseva et al. (2009); ${ }^{(d)}$ in $\mathrm{cm}^{-3}$; ${ }^{(e)}$ UVES data; ${ }^{(f)}$ medium-resolution FORS data; ${ }^{(g)}$ lowresolution FORS data. 

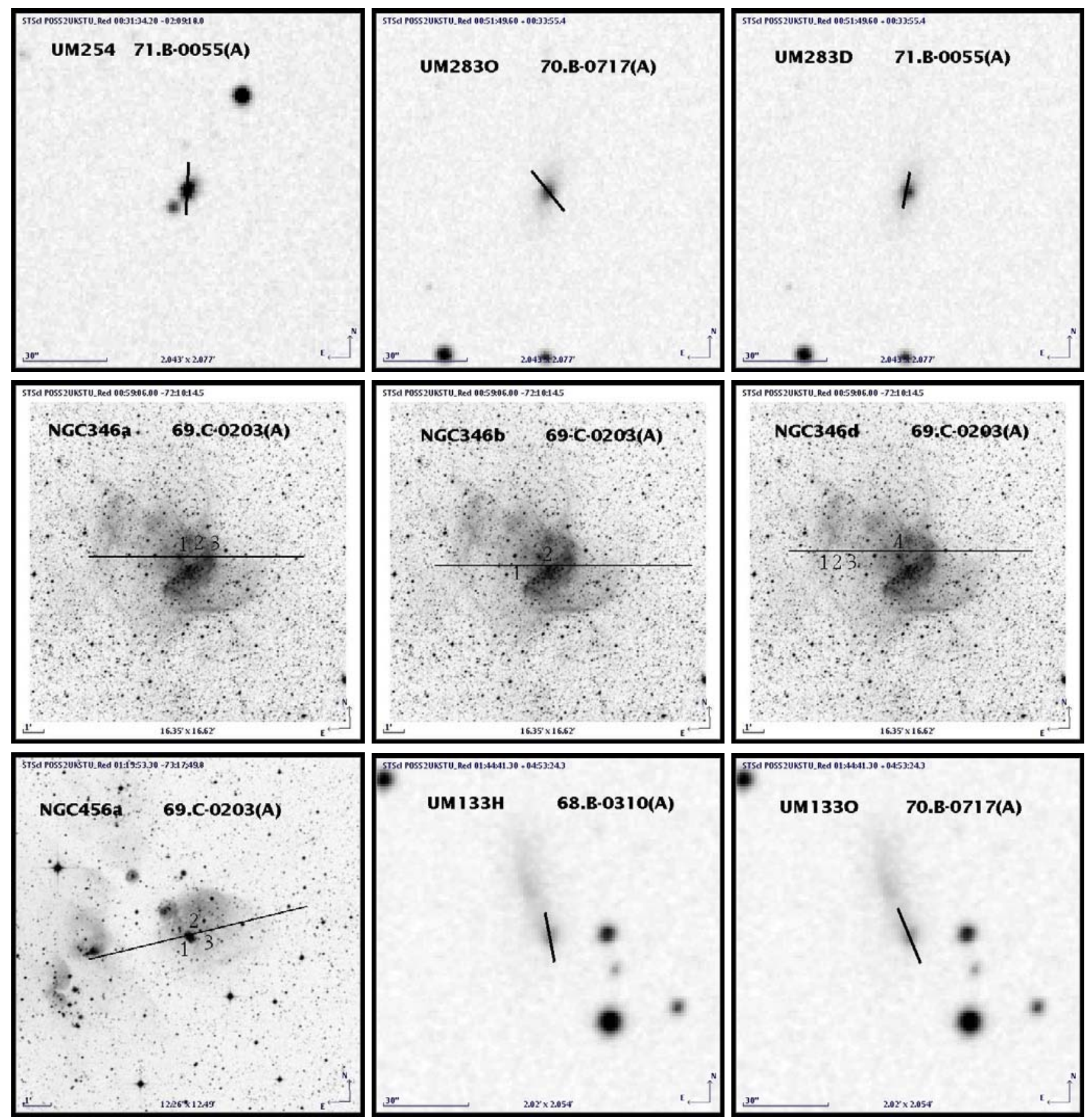

Fig. 1. Optical images of the galaxies. The straight lines indicate the location of the slit during observations (see Table 1). 
N. G. Guseva et al.: Low-metallicity ELGs: deep VLT/FORS+UVES spectroscopy
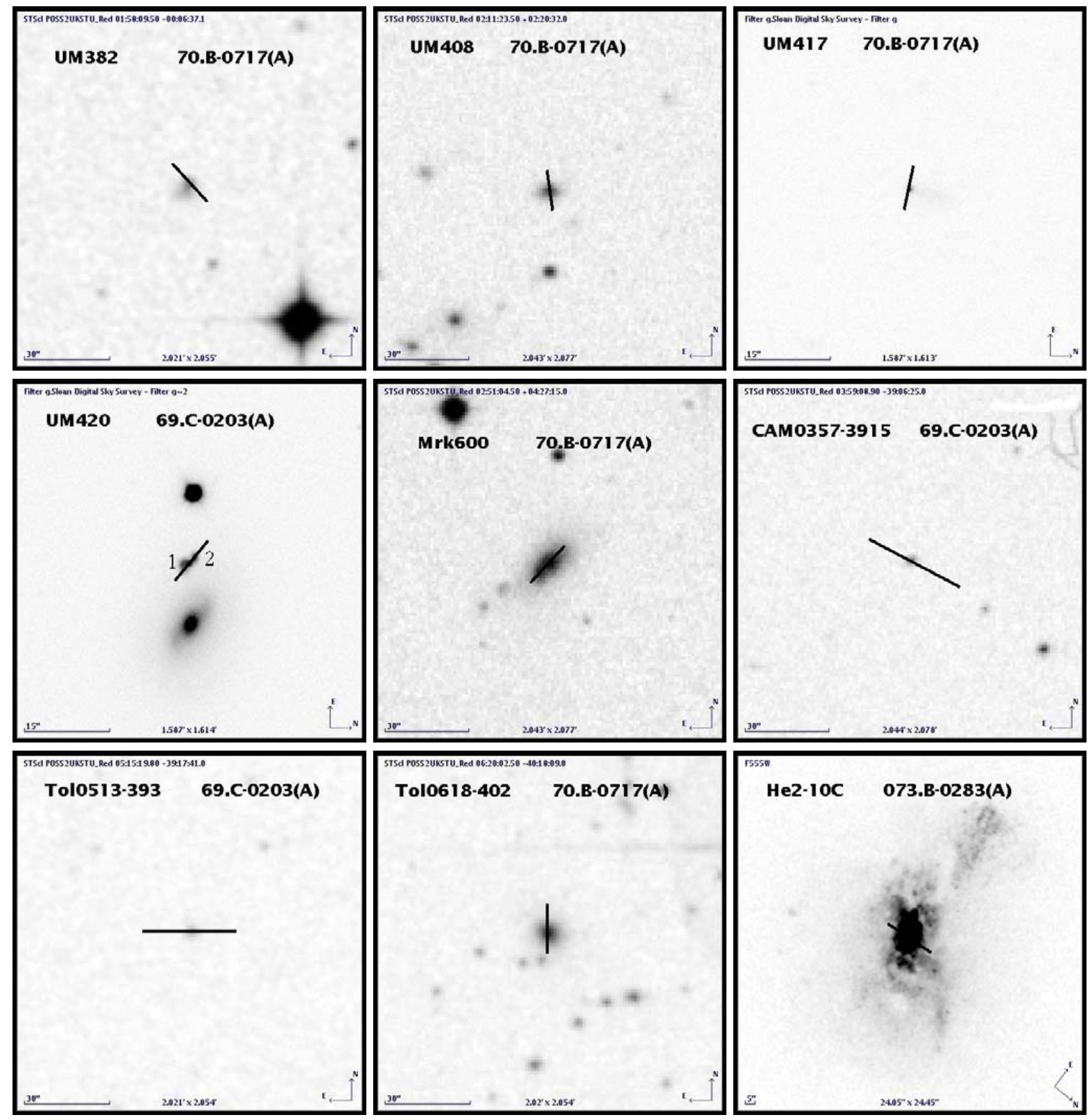

Fig. 1. continued. 
A\&A 529, A149 (2011)
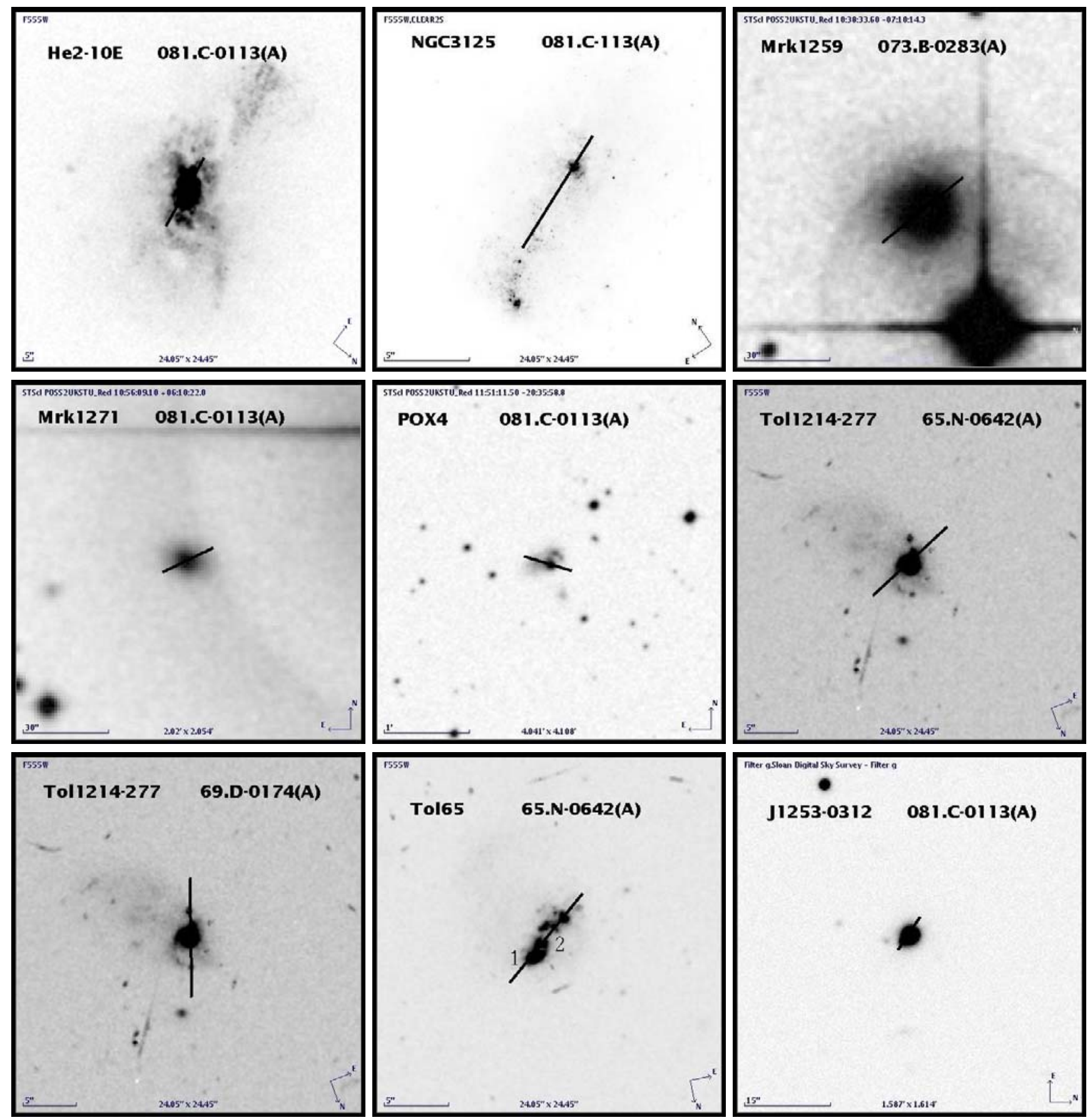

Fig. 1. continued. 
N. G. Guseva et al.: Low-metallicity ELGs: deep VLT/FORS+UVES spectroscopy
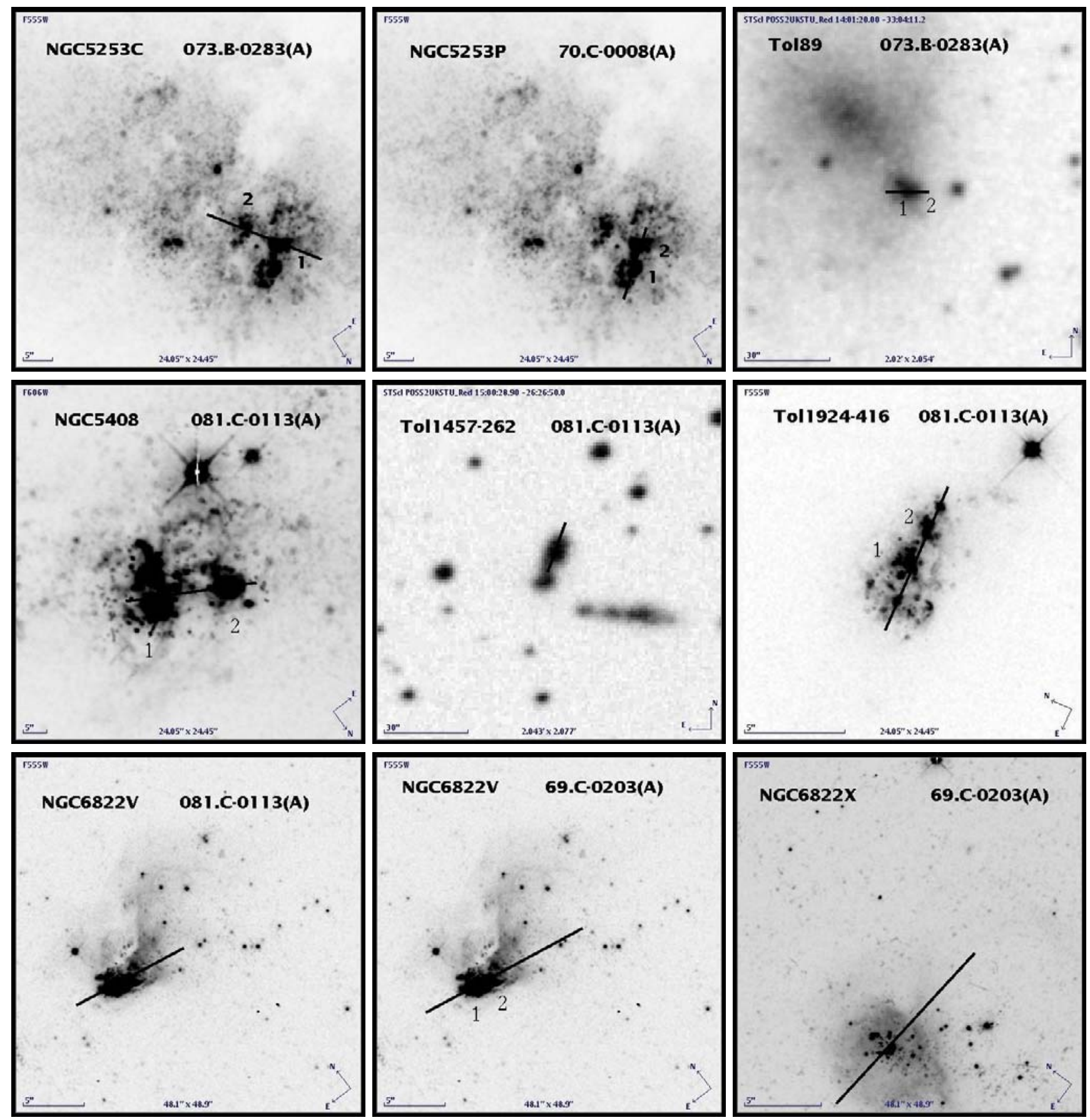

Fig. 1. continued. 
A\&A 529, A149 (2011)
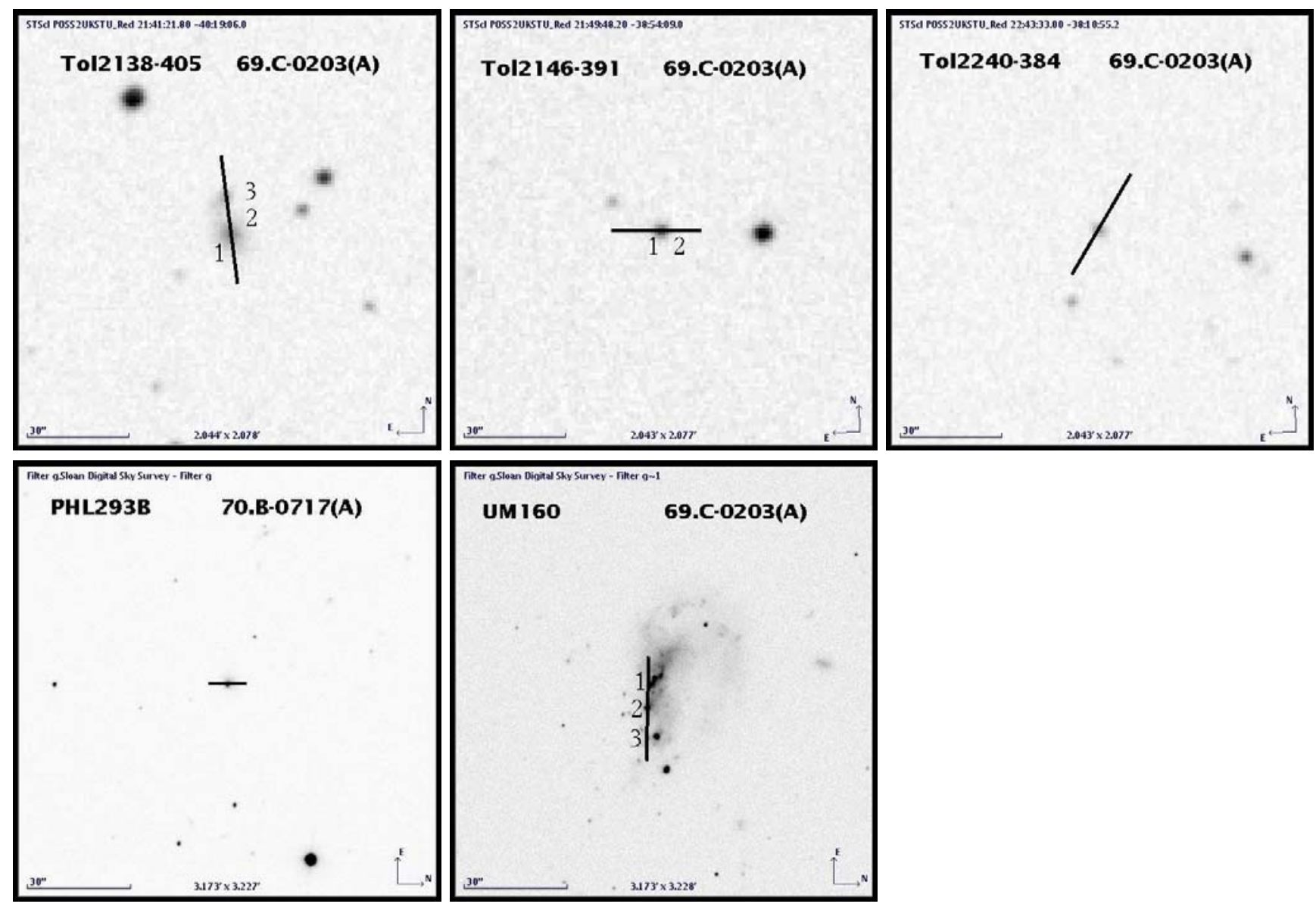

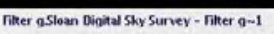

UM 160 69.C-0203(A)

Fig. 1. continued. 
N. G. Guseva et al.: Low-metallicity ELGs: deep VLT/FORS+UVES spectroscopy
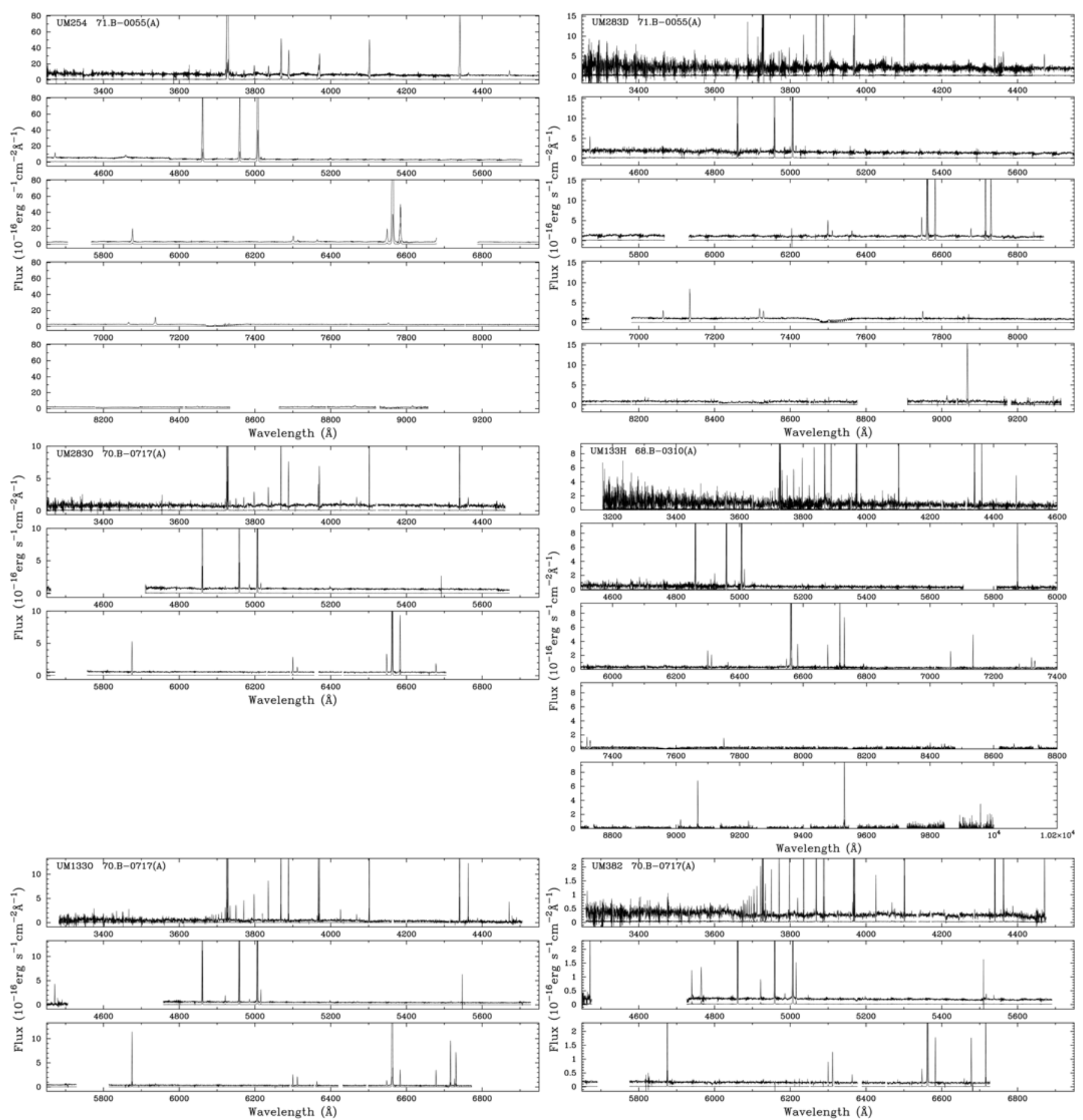

Fig. 2. Flux-calibrated and redshift-corrected UVES spectra of galaxies. 
A\&A 529, A149 (2011)
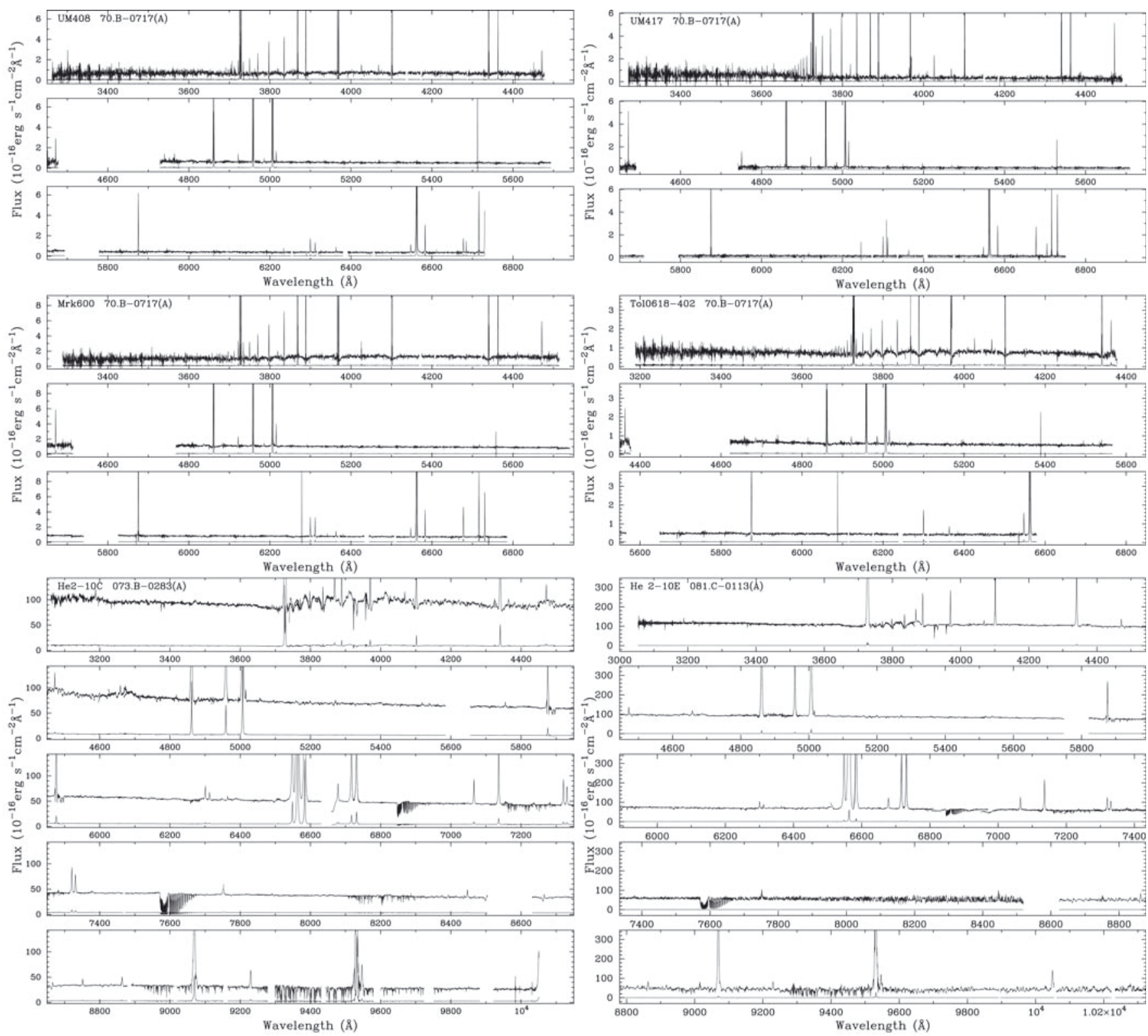

Fig. 2. continued. 
N. G. Guseva et al.: Low-metallicity ELGs: deep VLT/FORS+UVES spectroscopy
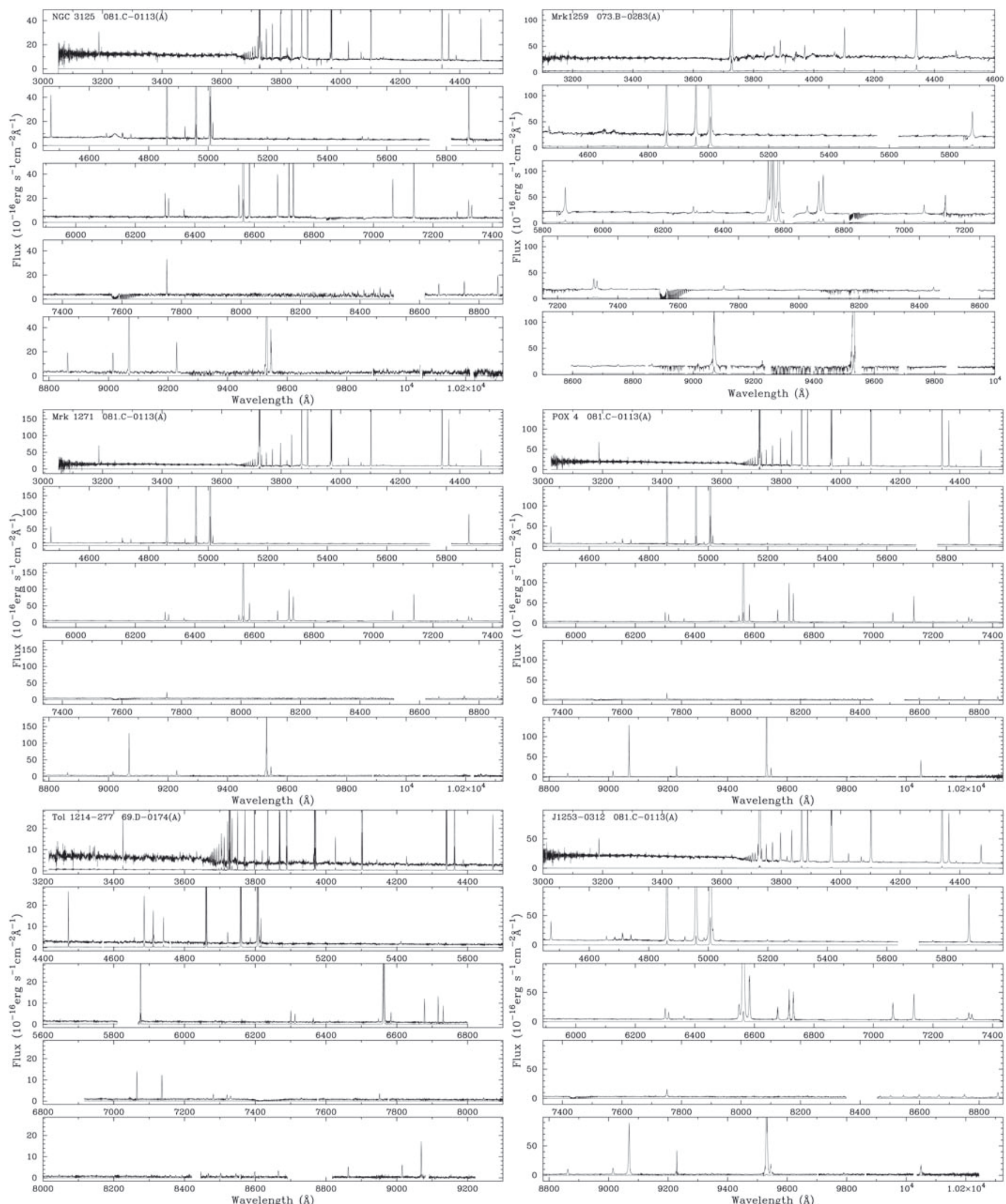

Fig. 2. continued. 
A\&A 529, A149 (2011)
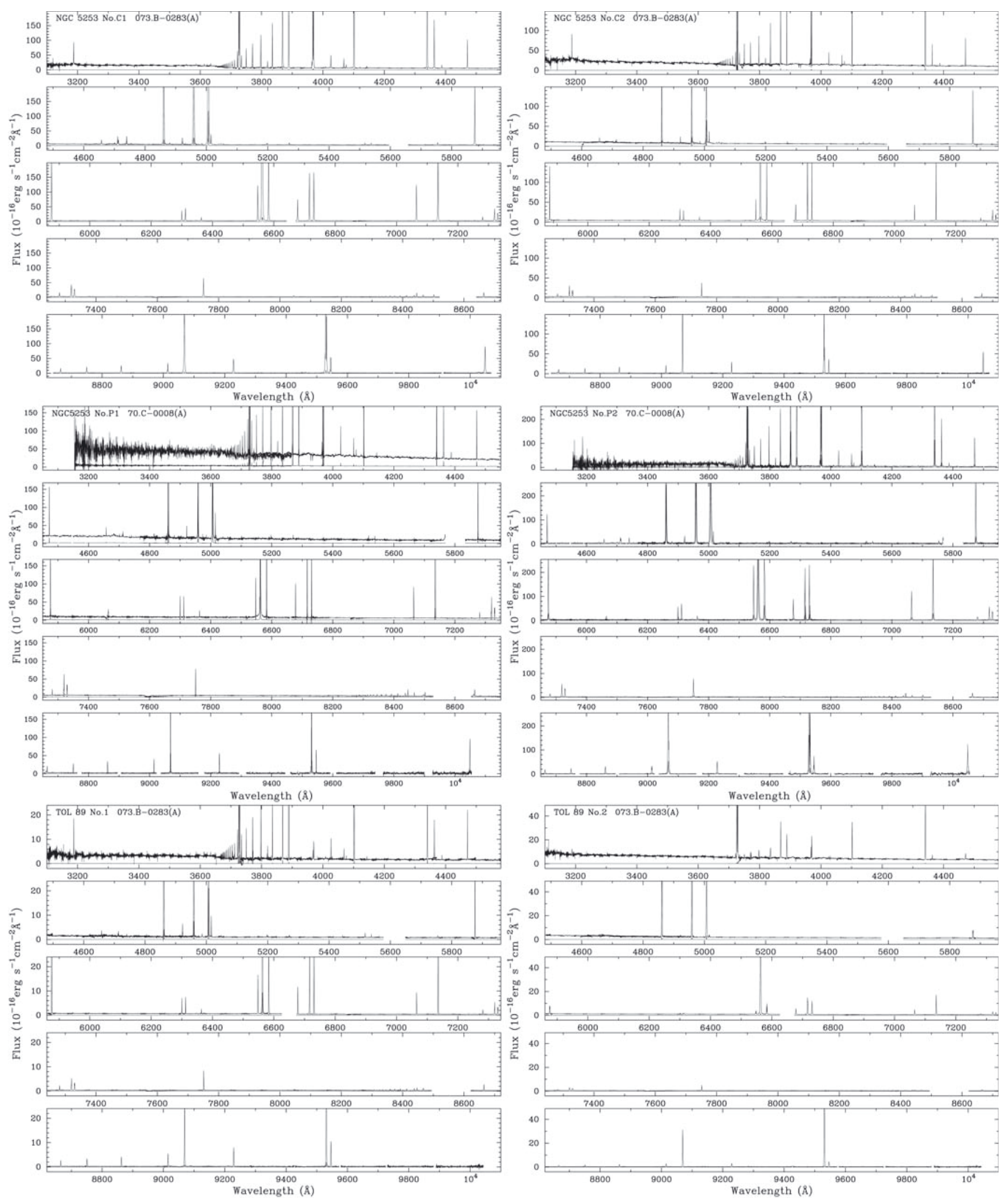

Fig. 2. continued.

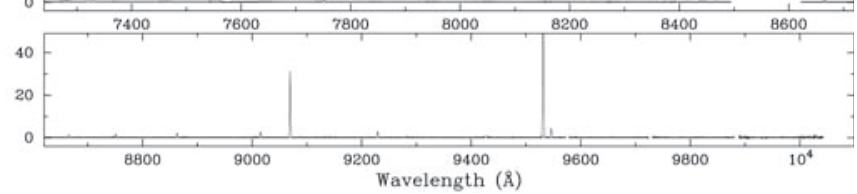


N. G. Guseva et al.: Low-metallicity ELGs: deep VLT/FORS+UVES spectroscopy
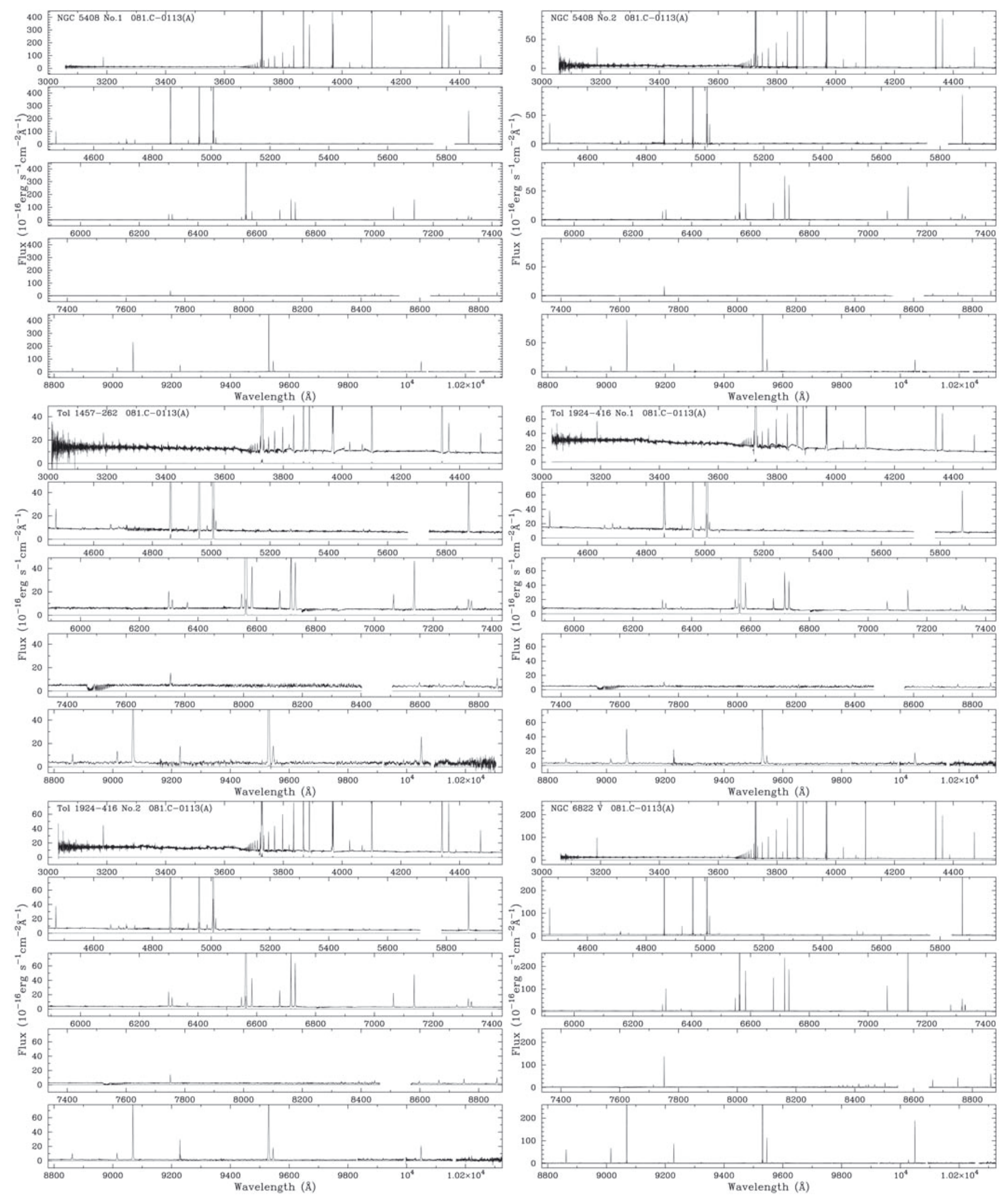

Fig. 2. continued. 


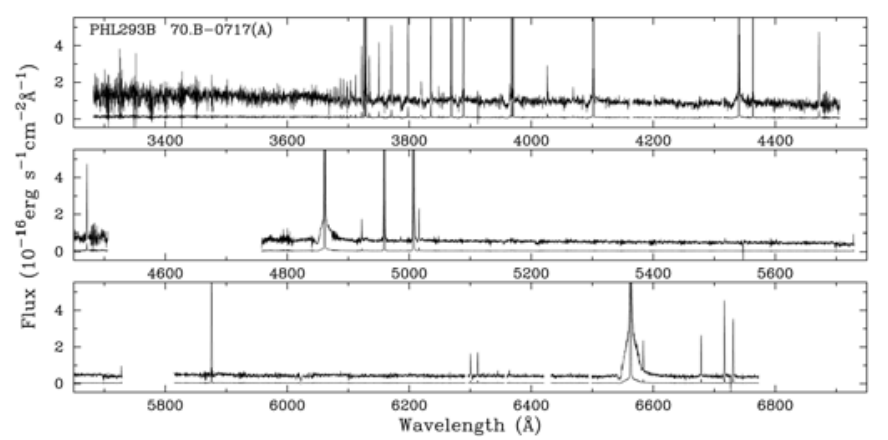

Fig. 2. continued.

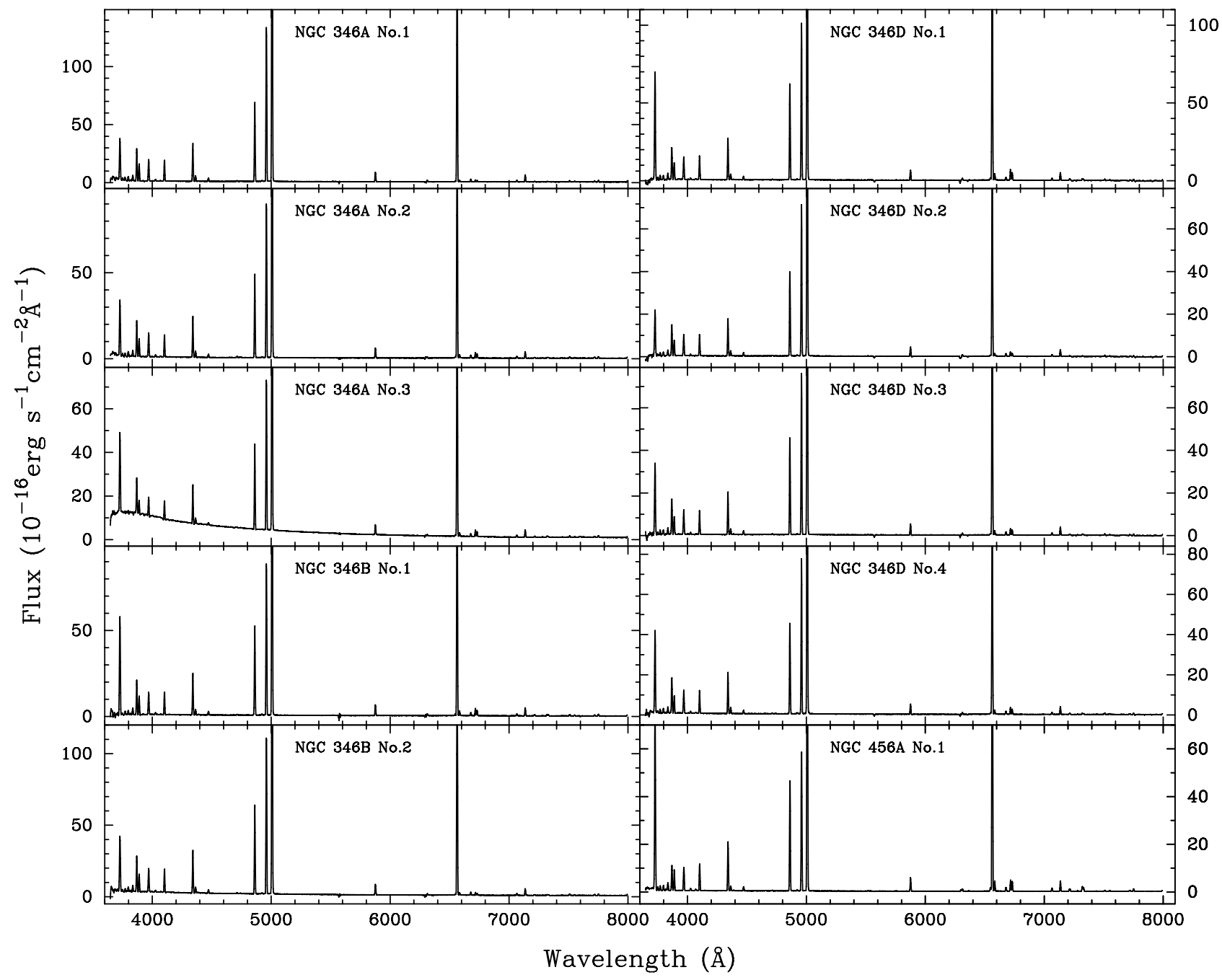

Fig. 3. Flux-calibrated and redshift-corrected FORS low-resolution spectra of galaxies. 
N. G. Guseva et al.: Low-metallicity ELGs: deep VLT/FORS+UVES spectroscopy

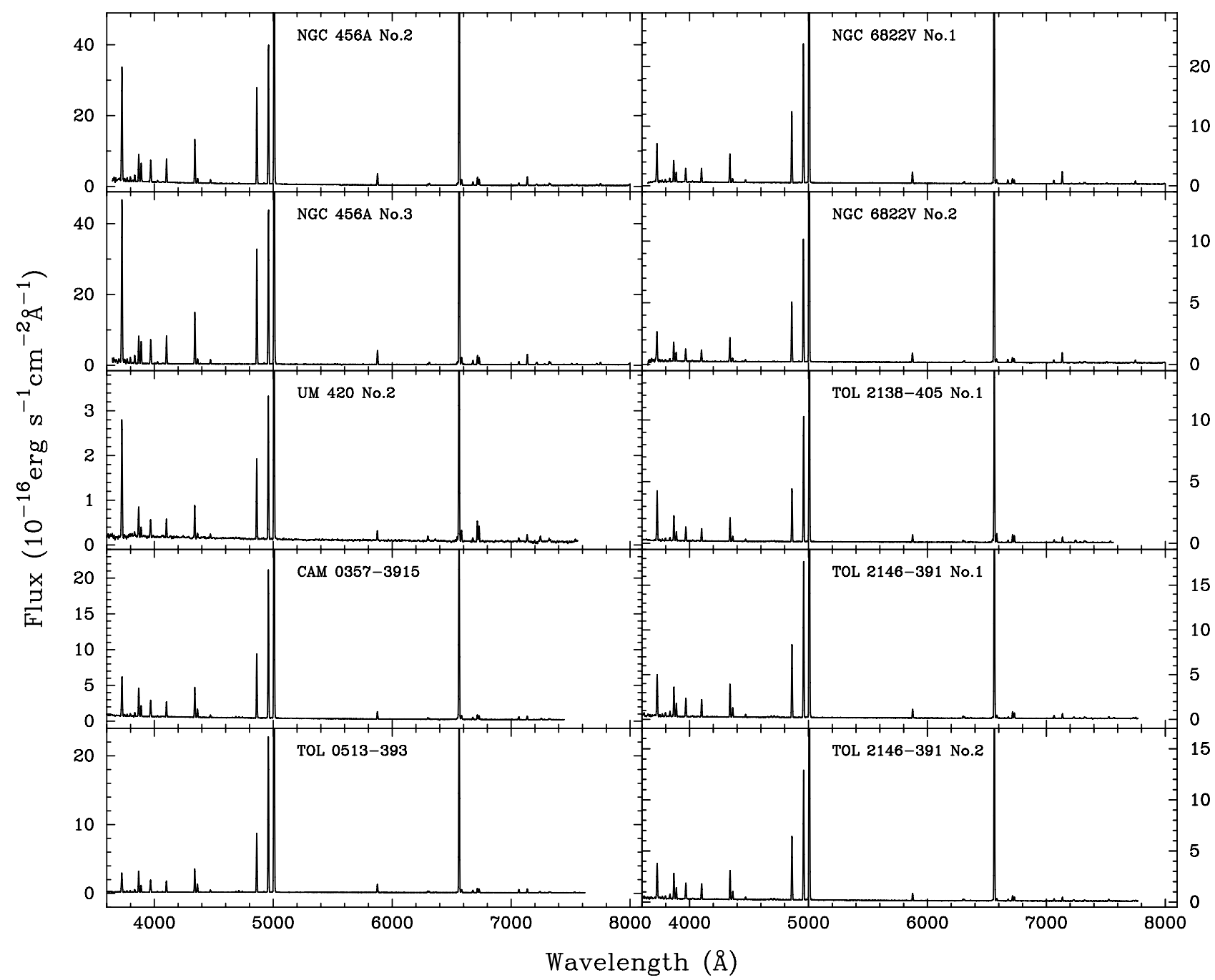

Fig. 3. continued.

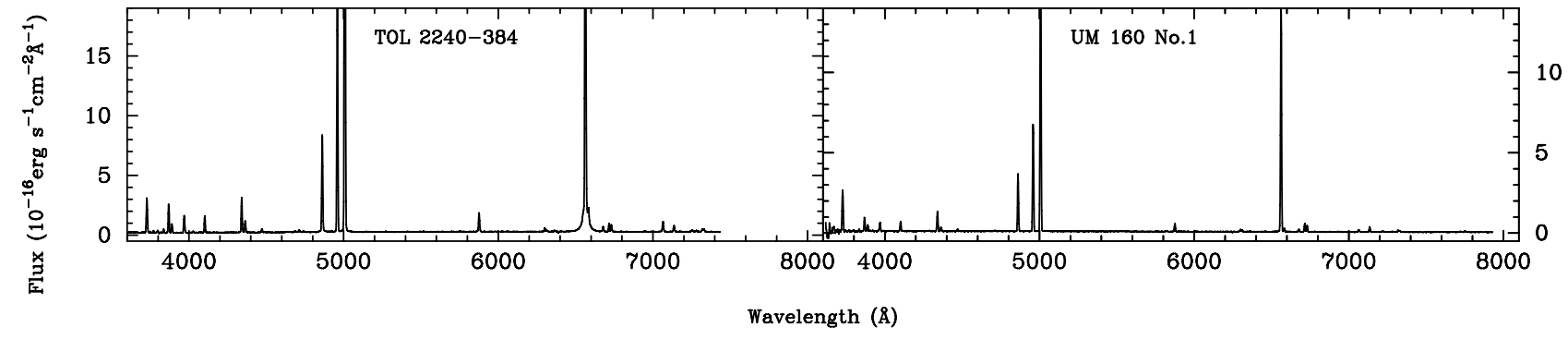

Fig. 3. continued. 
A\&A 529, A149 (2011)

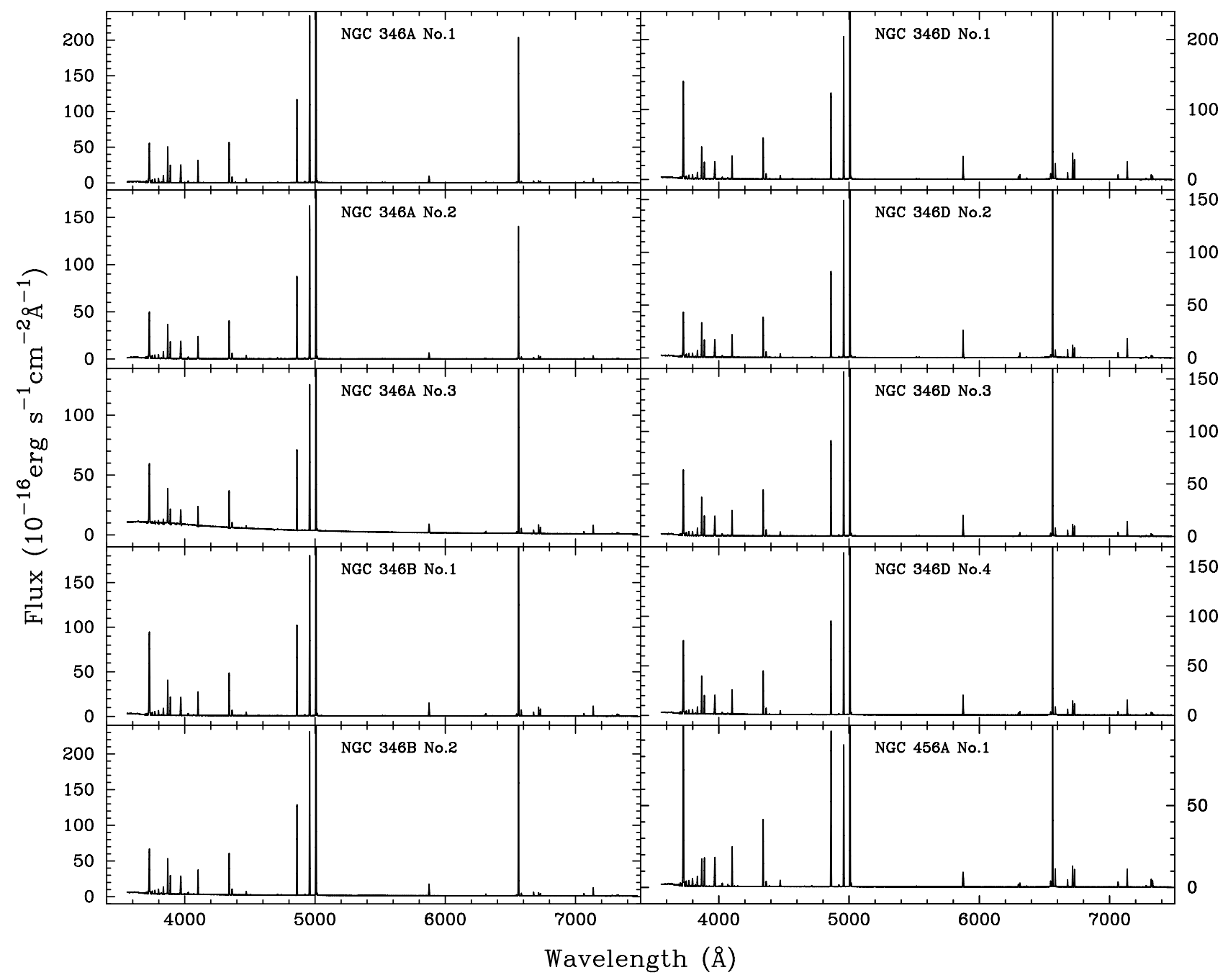

Fig. 4. Flux-calibrated and redshift-corrected FORS medium-resolution spectra of galaxies. 
N. G. Guseva et al.: Low-metallicity ELGs: deep VLT/FORS+UVES spectroscopy

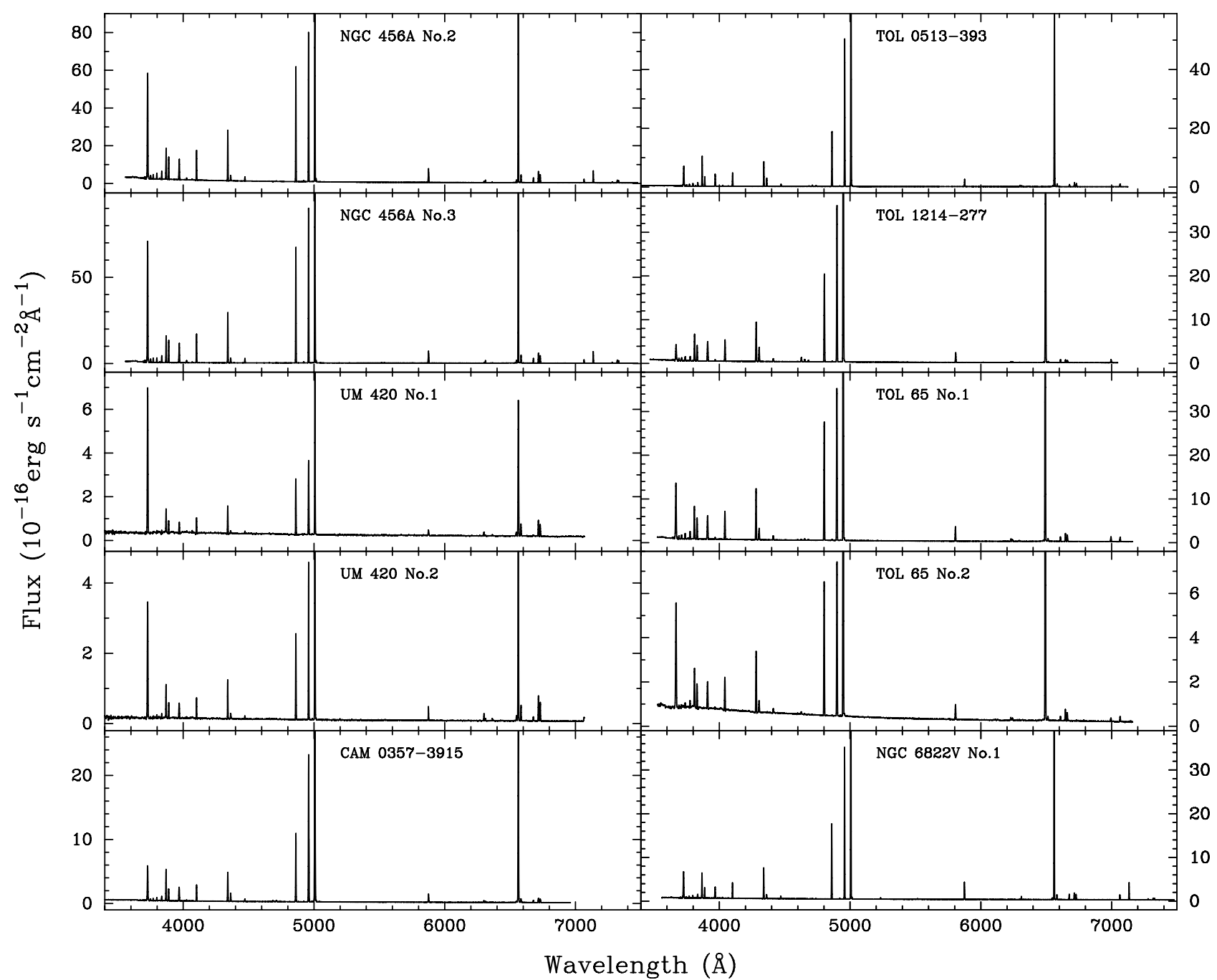

Fig. 4. continued. 
A\&A 529, A149 (2011)

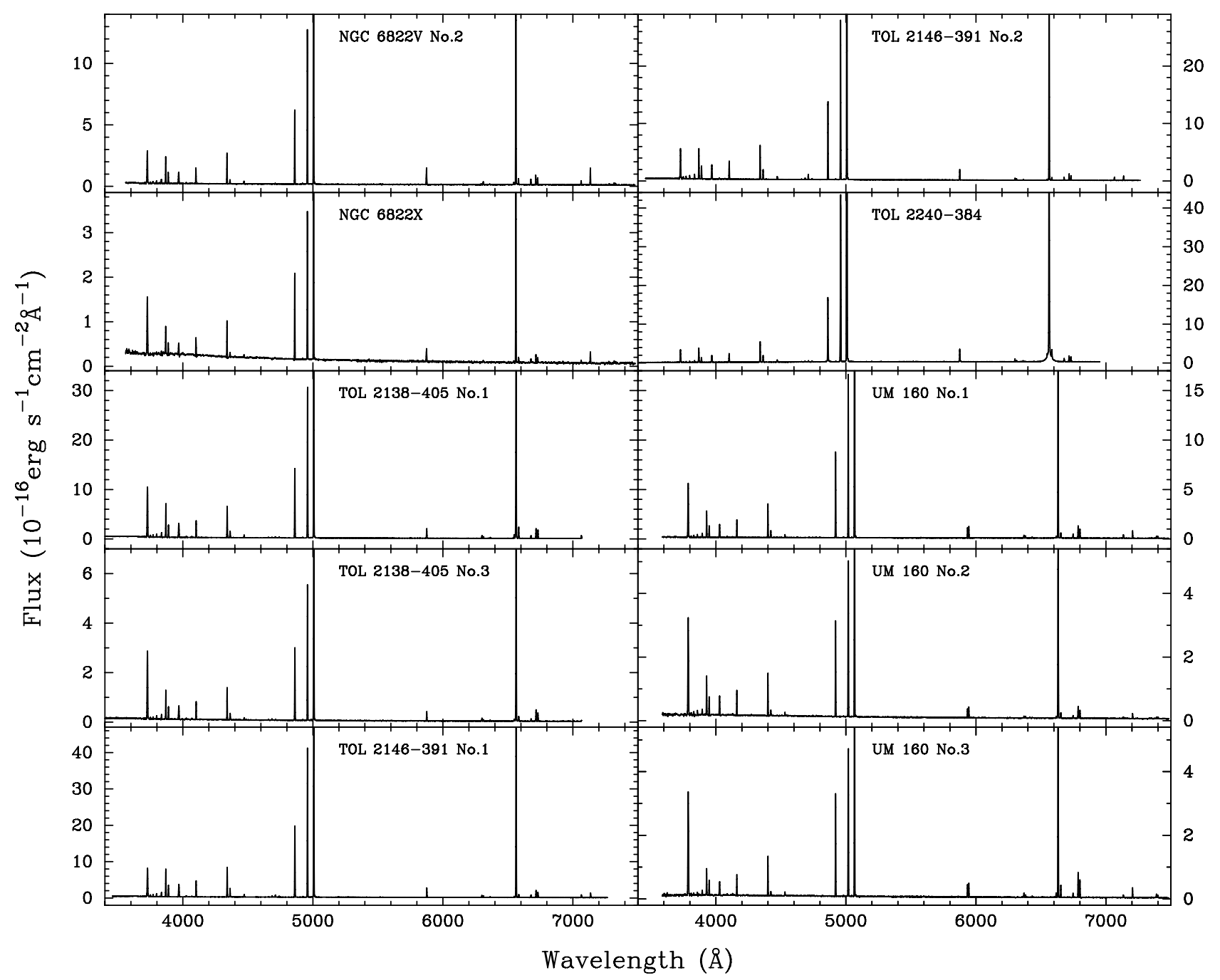

Fig. 4. continued. 